\title{
VIBRATION ANALYSIS OF A WIND TURBINE MULTI-STAGE PLANETARY GEARBOX INCORPORATING A FLEXIBLE BODY COMPONENT
}

\author{
A Thesis \\ presented to \\ the Faculty of California Polytechnic State University, \\ San Luis Obispo \\ In Partial Fulfillment \\ of the Requirements for the Degree \\ Master of Science in Mechanical Engineering \\ by \\ Tananant Boonya-ananta
}

December 2017 
(C) 2017

Tananant Boonya-ananta

ALL RIGHTS RESERVED 


\section{COMMITTEE MEMBERSHIP}

TITLE:

Vibration Analysis of a Wind Turbine Multi-Stage

Planetary Gearbox Incorporating a Flexible Body

Component

AUTHOR:

Tananant Boonya-ananta

DATE SUBMITTED: December 2017

COMMITTE CHAIR: $\quad$ Xi (Julia) Wu, Ph.D.

Professor of Mechanical Engineering

COMMITTEE MEMBER: $\quad$ Peter Schuster, Ph.D.

Professor of Mechanical Engineering

COMMITTEE MEMBER: $\quad$ Andrew Davol, Ph.D.

Professor of Mechanical Engineering 


\begin{abstract}
Vibration Analysis of a Wind Turbine Multi-Stage Planetary Gearbox Incorporating a Flexible Body Component
\end{abstract}

Tananant Boonya-ananta

The following thesis document researches into creating a model to represent the behavior of a wind turbine gearbox. This model is developed based on the overall parameters of a NORDEX N90 2.5MW wind turbine developed by a German company Nordex SE. This research focuses on the combination of a flexible body and a multibody dynamics analysis software. This is done through the usage of MSC ADAMS, a multibody dynamic analysis program, and MSC

Patran/Nastran, a finite element analysis software, and its associated solver. The model is created to show the vibration patterns of a healthy gearbox with rigid bodies, with a flexible body, and with a defect applied on a particular gear in the planetary gear systems that is representative of the N90 wind turbine. The flexible body incorporation allows for stress analysis of different gear teeth at different locations.

Keywords: Gearbox, Nordex N90, Wind turbine, Fast Fourier Transform (FFT), Gear Mesh Frequency (GMF), Planetary Gearbox, Vibration Pattern 


\section{ACKNOWLEDGMENTS}

I like to extend my appreciation to Doug Malcolm from MSC Software Corporation who was instrumental to the development of the working finite element model. I would like to also express my gratitude to Dr. Wu for her guidance and advise as well as Dr. Schuster and Dr. Davol for their input. 


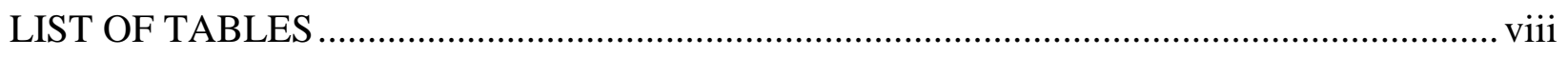

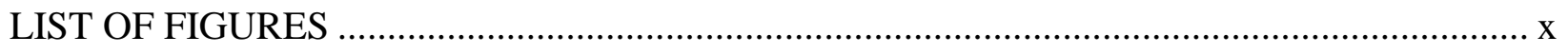

CHAPTER 1. INTRODUCTION AND LITERATURE REVIEW …......................................... 1

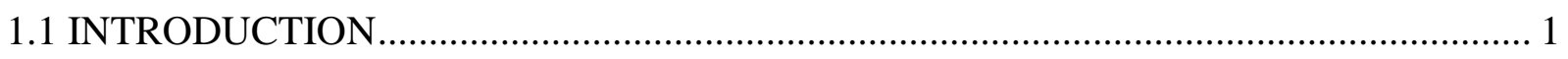

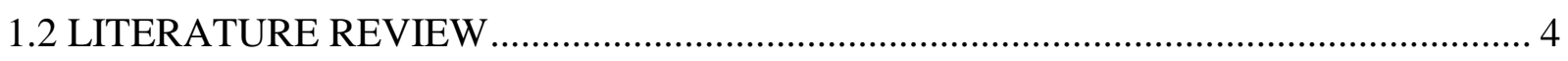

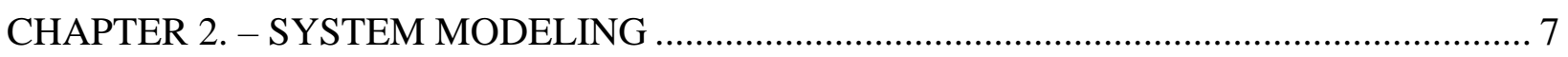

2.1 SYSTEM MODELING - SOLIDWORKS COMPUTER AIDED DESIGN ........................ 7

CHAPTER 3. MSC PATRAN/NASTRAN FINITE ELEMENT MODEL …………….................. 16

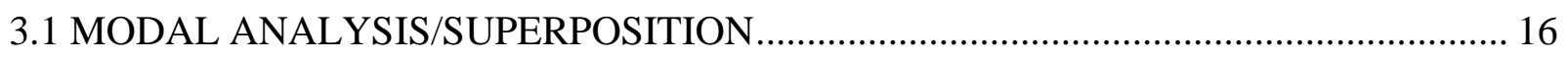

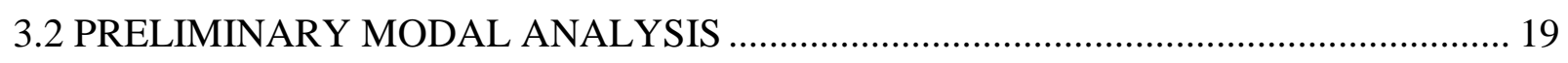

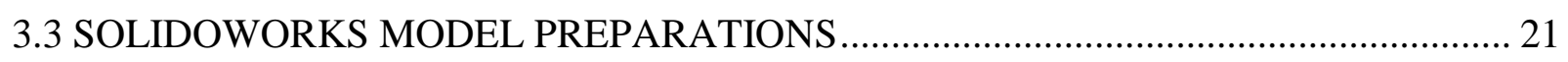

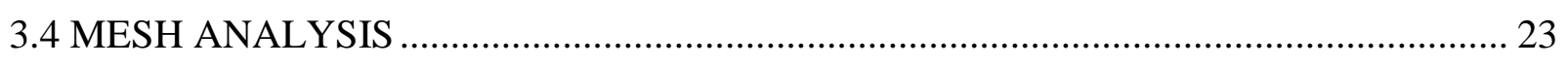

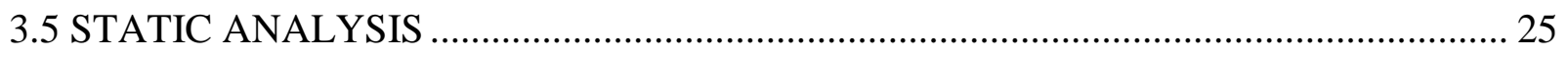

3.6 PATRAN FINITE ELEMENT MODEL …………………………………………...... 26

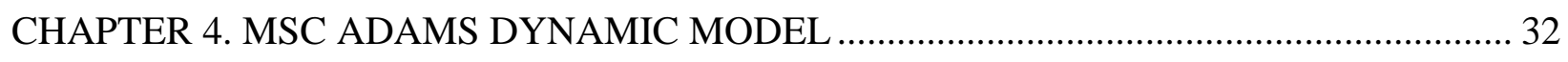

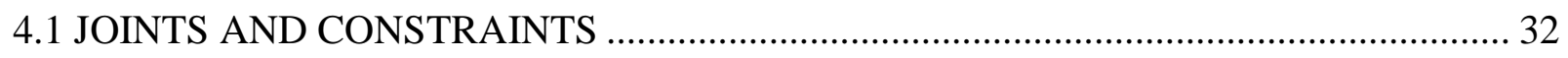

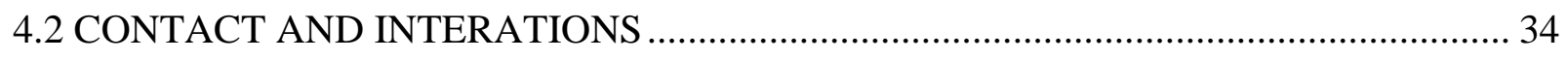

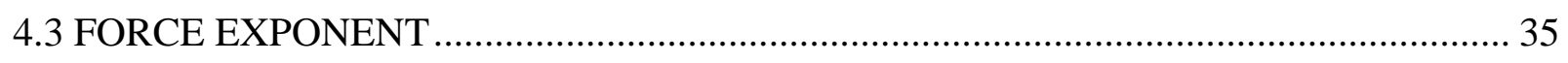

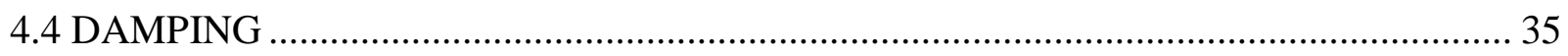

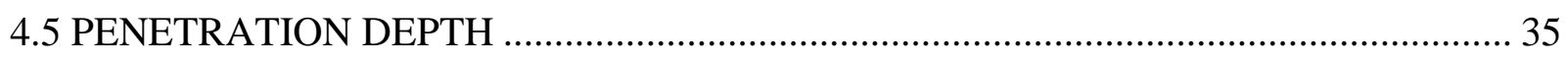

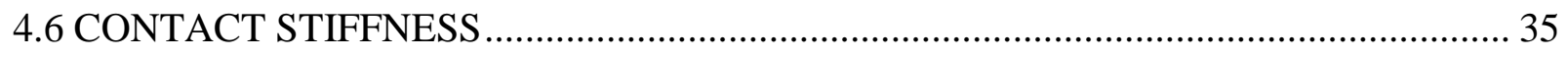

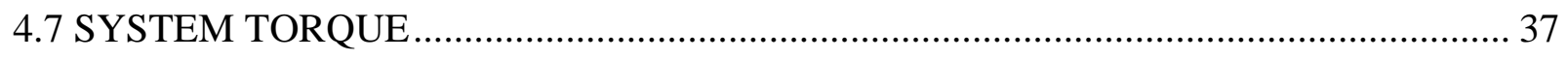

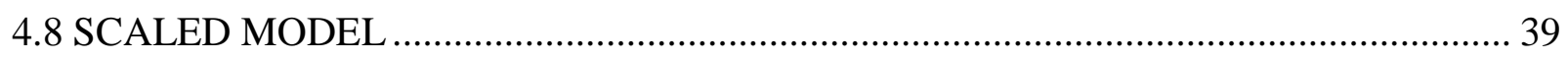

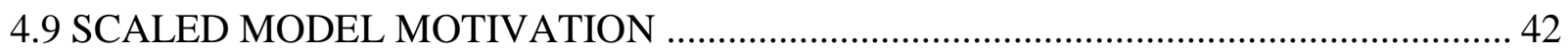

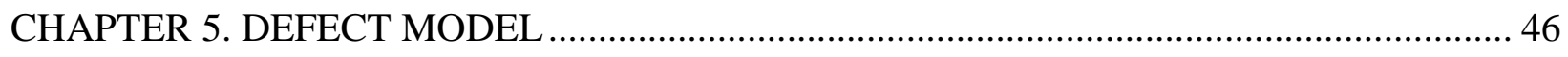

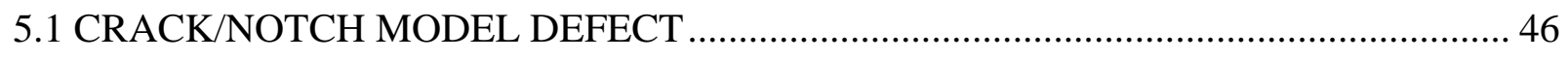

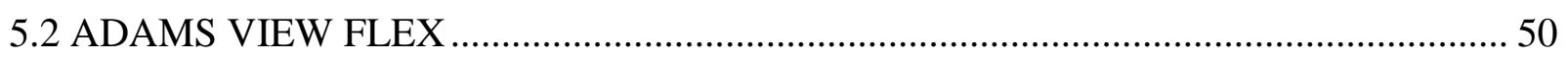

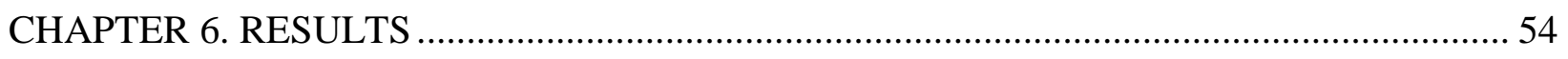

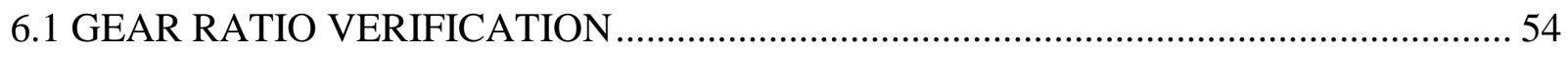

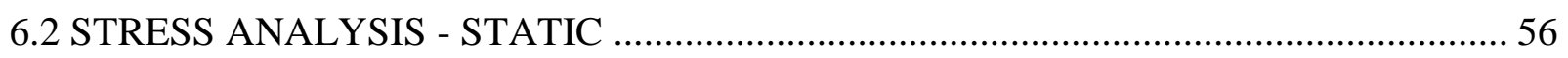




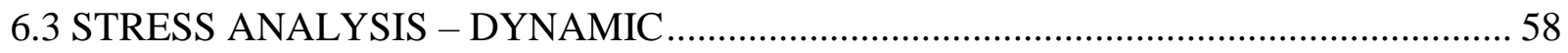

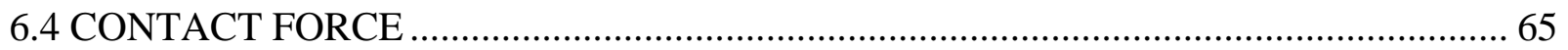

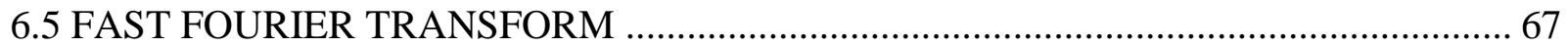

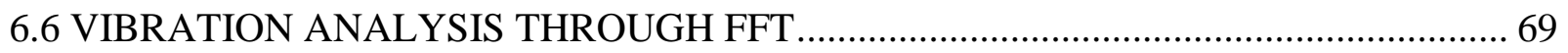

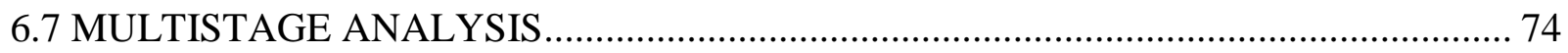

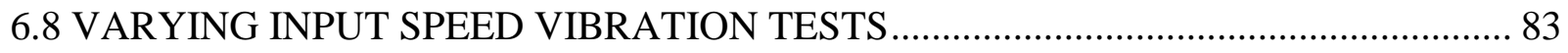

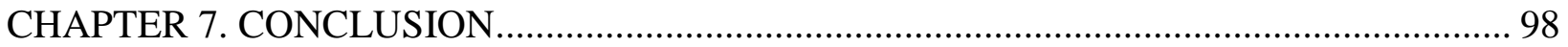

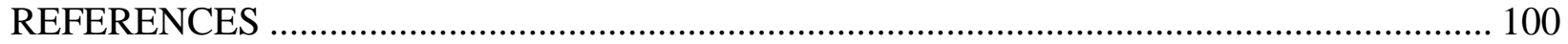

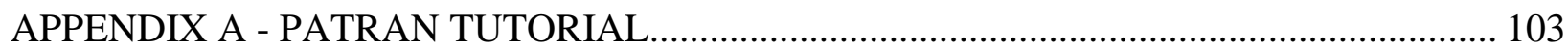

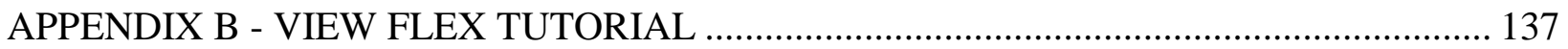




\section{LIST OF TABLES}

Page

Table 1. Gearbox components design parameters ………..................................................

Table 2. Scaled model parameters ……………………..................................................14

Table 3. Mesh Convergence Study for Stress with Varying Seed Size ..................................24

Table 4. FEA model comparison with cantilever beam........................................................26

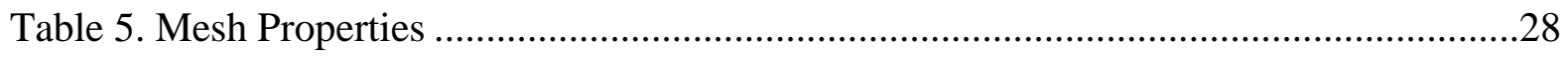

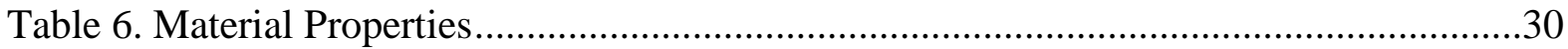

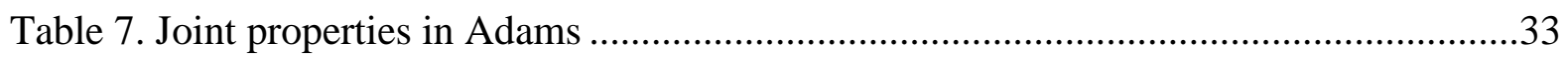

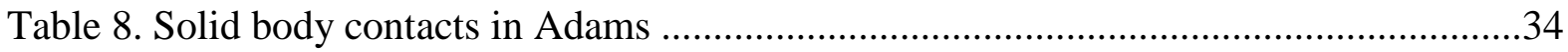

Table 9. Torque and System Power ....................................................................................

Table 10. Half Scale Solid Body Joints .............................................................................

Table 11. Calculated Component Velocities …………….................................................5

Table 12. Static Analysis of a Sun Gear Tooth with varying defect size. ................................57

Table 13. Stresses at six node locations of the gear tooth from the center to the face ............63

Table 14. FFT Stage 1 contact force between sun and planet gear frequency amplitudes .......70

Table 15. Comparison between amplitudes at the GMF harmonics of Stage 1 with and without a defect at the sun gear tooth.................................................................72

Table 16. Comparison between amplitudes at the GMF harmonics of Stage 1 with and without a defect at the sun gear tooth using flexible bodies. ....................................74

Table 17. Comparison between amplitudes at the GMF harmonics of Stage 1 with and without a defect at the sun gear tooth..................................................................78

Table 18. Comparison between amplitudes at the GMF harmonics of Stage 2 with and without a defect at the sun gear tooth. 
Table 19. Comparison between amplitudes at the GMF harmonics of Stage 3 with and

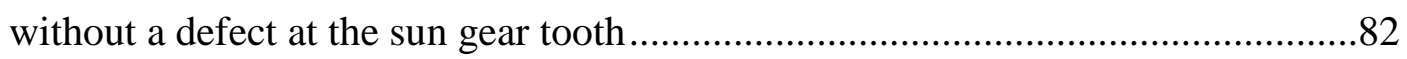

Table 20. Base Gear Mesh Frequencies for different speed input ............................................83 


\section{LIST OF FIGURES}

Page

Figure 1. Gear tooth involute profile .............................................................................. 7

Figure 2. Gear tooth profile development in Solidworks .................................................... 8

Figure 3. Gear tooth rotation to create full gear............................................................... 8

Figure 4. Full isometric Solidworks Assembly........................................................... 12

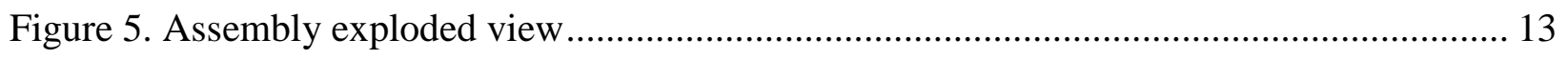

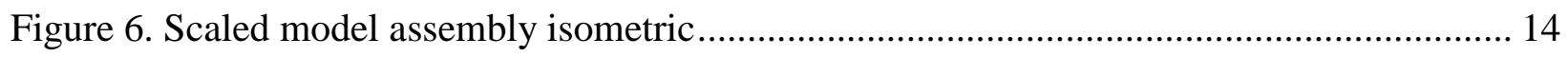

Figure 7. First three bending modes for a beam with Fixed-Fixed boundary conditions [25] ..... 17

Figure 8. Simple beam with two constraint modes ........................................................ 18

Figure 9. First vibration mode for the first stage sun gear.............................................. 20

Figure 10. Eleventh vibration mode for the first stage sun gear ....................................... 20

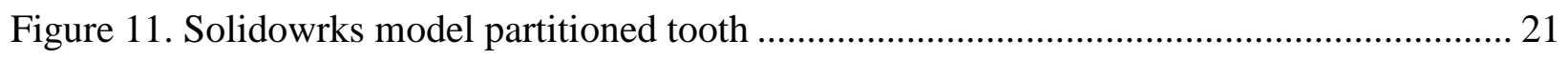

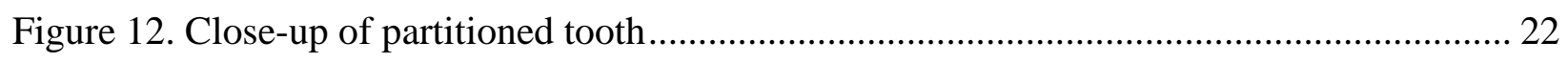

Figure 13. Meshed tooth for convergence study ........................................................... 24

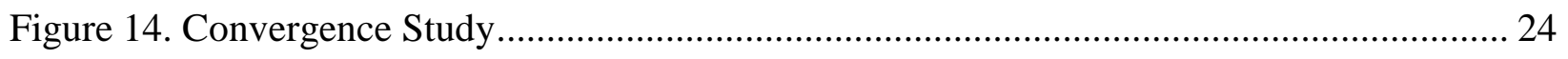

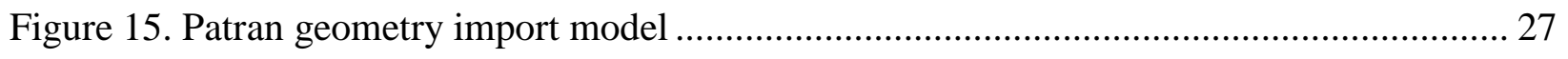

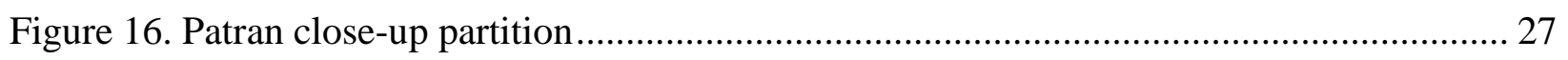

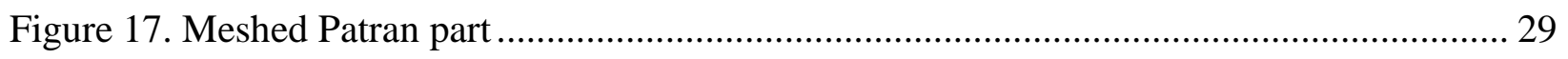

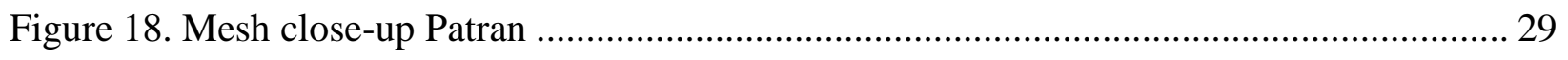

Figure 19. RBE2 Spider constraint of gear body ............................................................ 31

Figure 20. Torque and System Power as a function of wind speed ...................................... 37

Figure 21. Full Assembly Half Scale Model - Solidworks Render....................................... 39

Figure 22. Full Assembly Half Scaled Model - Adams ..................................................... 40 
Figure 23. Adams model with joint locations ............................................................. 42

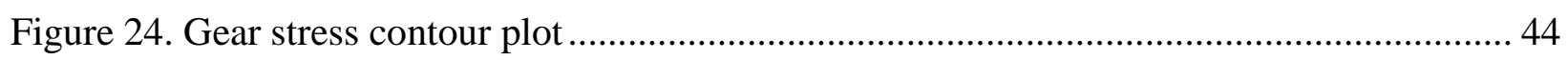

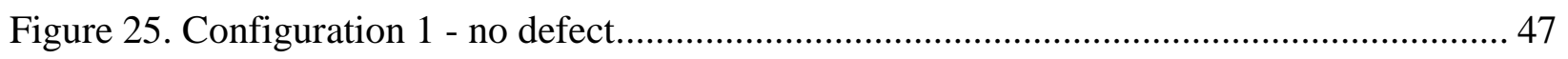

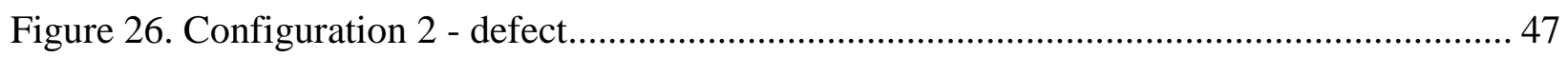

Figure 27. Notch radius mesh refinement at the sun gear of the first stage............................ 48

Figure 28. Opposite side root mesh for compressive stress analysis .................................. 48

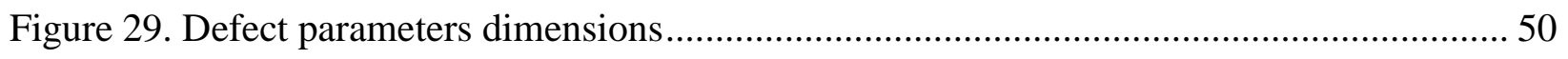

Figure 30. Adams Flex Control Module View ............................................................... 52

Figure 31. Stage 1 and Stage 2 Component Velocities..................................................... 55

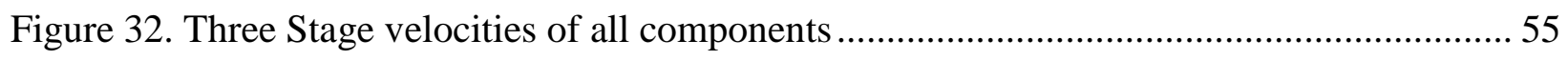

Figure 33. AISI 4820 Steel Estimated Non-Linear Stress vs. Strain Relationship.................... 56

Figure 34. 25\% Root Notch Deformed Body Front View .................................................. 57

Figure 35. 25\% Root Notch Deformed Body Notch View .................................................. 58

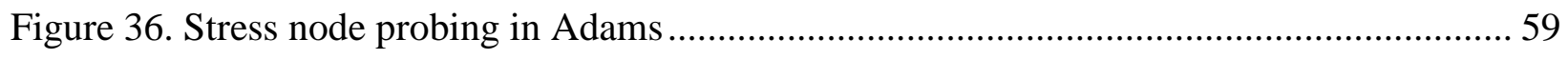

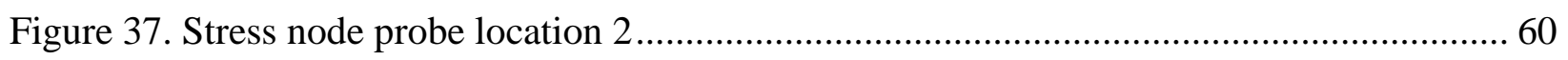

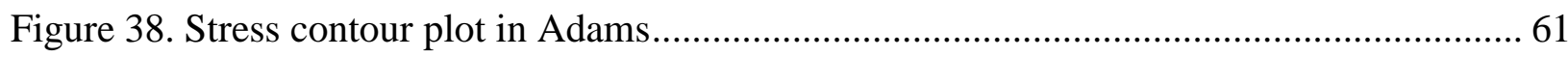

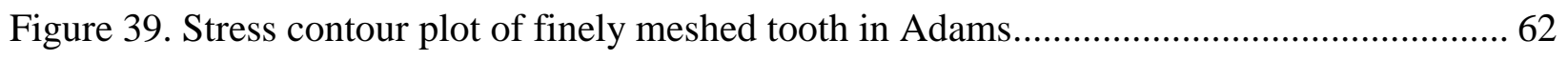

Figure 40. Minimum principal, compressive, stress from the root center to the face ................ 63

Figure 41.Maximum principal, tensile, stress from the root center to the face ........................ 64

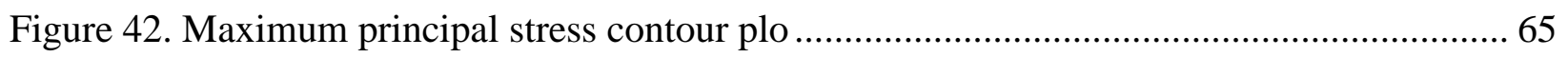

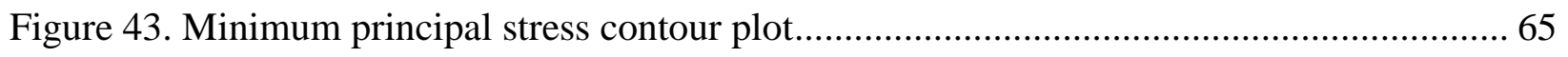

Figure 44. Gear Contact Force Stage 1 Sun to Planets with Flexible Body.............................. 66

Figure 45. Gear Contact Force Stage 1 Sun to Planets without Flexible Body......................... 67 
Figure 46. A signal viewed in frequency and time domain [23] 68

Figure 47. Contact force FFT sun to planet Stage 1 70

Figure 48. Stage 1 contact force FFT between sun and planet gears with a defect on the sun gear

tooth. 71

Figure 49. Stage 1 superposition comparison FFT of the contact force between the sun and planet gear with and without a defect at the sun gear tooth. 72 Figure 50. Stage 1 superposition comparison FFT of the contact force between the sun and planet gear with and without a defect at the sun gear tooth using flexible bodies. 73 Figure 51. FFT of Stage 1 contact force between sun and planet gear in a full three stage assembly. 75

Figure 52. FFT of Stage 2 contact force between sun and planet gear in a full three stage assembly. 76

Figure 53. FFT of Stage 3 contact force between sun and planet gear in a full three stage assembly. 76

Figure 54. FFT of Stage 1 contact force between sun and planet gear in a full three stage assembly with a defect at first stage sun gear. 77 Figure 55. Stage 1 superposition comparison FFT of the contact force between the sun and planet gear with and without a defect at the sun gear tooth. 78 Figure 56. FFT of Stage 2 contact force between sun and planet gear in a full three stage assembly with a defect at first stage sun gear. 79 Figure 57. Stage 2 superposition comparison FFT of the contact force between the sun and planet gear with and without a defect at the sun gear tooth. 80 
Figure 58. FFT of Stage 3 contact force between sun and planet gear in a full three stage assembly with a defect at first stage sun gear. 81

Figure 59. Stage 3 superposition comparison FFT of the contact force between the sun and planet gear with and without a defect at the sun gear tooth. 82 Figure 60. FFT of Stage 1 contact force between sun and planet gear in a full three stage assembly with input speed $1.5 \mathrm{rad} / \mathrm{sec}$. 84 Figure 61. FFT of Stage 1 contact force between sun and planet gear in a full three stage assembly with a defect at first stage sun gear with input speed $1.5 \mathrm{rad} / \mathrm{sec}$. 84 Figure 62. Stage 1 superposition comparison FFT of the contact force between the sun and planet gear with and without a defect at the sun gear tooth with input speed $1.5 \mathrm{rad} / \mathrm{sec}$. 85 Figure 63. FFT of Stage 2 contact force between sun and planet gear in a full three stage assembly with input speed $1.5 \mathrm{rad} / \mathrm{sec}$. 86

Figure 64. FFT of Stage 2 contact force between sun and planet gear in a full three stage assembly with a defect at first stage sun gear with input speed $1.5 \mathrm{rad} / \mathrm{sec}$. 86 Figure 65. Stage 2 superposition comparison FFT of the contact force between the sun and planet gear with and without a defect at the sun gear tooth with input speed $1.5 \mathrm{rad} / \mathrm{sec}$. 87 Figure 66. FFT of Stage 3 contact force between pinion and gear in a full three stage assembly with input speed $1.5 \mathrm{rad} / \mathrm{sec}$. 88 Figure 67. FFT of Stage 3 contact force between pinion and gear in a full three stage assembly with a defect at first stage sun gear with input speed $1.5 \mathrm{rad} / \mathrm{sec}$. 88 Figure 68. Stage 3 superposition comparison FFT of the contact force between the pinion and gear with and without a defect at the sun gear tooth with input speed $1.5 \mathrm{rad} / \mathrm{sec}$. 89 
Figure 69. FFT of Stage 1 contact force between sun and planet gear in a full three stage assembly with input speed $2.0 \mathrm{rad} / \mathrm{sec}$.

Figure 70. FFT of Stage 1 contact force between sun and planet gear in a full three stage assembly with a defect at first stage sun gear with input speed $2.0 \mathrm{rad} / \mathrm{sec}$. 91 Figure 71. Stage 1 superposition comparison FFT of the contact force between the sun and planet gear with and without a defect at the sun gear tooth with input speed $2.0 \mathrm{rad} / \mathrm{sec}$. 91 Figure 72. FFT of Stage 2 contact force between sun and planet gear in a full three stage assembly with input speed $2.0 \mathrm{rad} / \mathrm{sec}$.

Figure 73. FFT of Stage 2 contact force between sun and planet gear in a full three stage assembly with a defect at first stage sun gear with input speed $2.0 \mathrm{rad} / \mathrm{sec}$.

Figure 74. Stage 2 superposition comparison FFT of the contact force between the sun and planet gear with and without a defect at the sun gear tooth with input speed $2.0 \mathrm{rad} / \mathrm{sec}$ 94

Figure 75. FFT of Stage 3 contact force between pinion and gear in a full three stage assembly with input speed $2.0 \mathrm{rad} / \mathrm{sec}$.

Figure 76. FFT of Stage 3 contact force between pinion and gear in a full three stage assembly with a defect at first stage sun gear with input speed $2.0 \mathrm{rad} / \mathrm{sec}$. 96 Figure 77. Stage 3 superposition comparison FFT of the contact force between the pinion and gear with and without a defect at the sun gear tooth with input speed $2.0 \mathrm{rad} / \mathrm{sec}$. 96 


\section{CHAPTER 1. INTRODUCTION AND LITERATURE REVIEW}

\subsection{INTRODUCTION}

Vibration analysis has been a core area of focus in the field of mechanical engineering for decades. Vibrations exists in many varying scopes from vibrations of molecules to the vibration of structures. The field of vibrations emphasizes on analyzing the frequencies at which objects oscillate. A particular frequency at which vibrations revolves around is the natural frequencies of a system, these are the frequencies at which an object tends to oscillate. Oscillation about an undamped natural frequency is referred to as resonance, where the amplitude of oscillation can be significantly greater than that of a periodic input frequency. The resulting effect of resonance can cause catastrophic failures of structures or mechanisms.

In the analysis of rotating machinery, vibration patterns can be studied closely to determine health of a particular system. In this case the gearbox of mechanical drive trains used to power a vast variety of different mechanisms throughout the world also exhibits particular frequencies which can be analyzed in order to determine the conditions of the gearbox without having to fully disassemble the system. This is an advantageous method of monitoring the health of machinery so as to be able to prevent critical failure of components which could very costly or endanger lives.

There exists different vibration patterns for different types of components inside a gearbox. These patterns can be created by the shaft, bearings, or, but not limited to, the gears themselves. The gear meshes exhibit a vibration unique to the number of teeth between the meshing components and the speeds at which they rotate. Wear and damage to gears will create a different vibration pattern when compared to a baseline pattern. This output can be monitored in 
order to protect the life of the gearbox and the mechanical system. A gear can be determined as damaged and replaced before the damage propagates to the system as a whole.

A model of a gear system can be simulated in a program called MSC Adams which is identified as a multibody dynamic analysis software. Adams allows for the creating of different types of kinetic joints and body contacts for creation of a dynamic system. A dynamic model allows for simulation of real world interactions which some other type of analyses may not offer. This pertains to a finite element analysis (FEA). FEA is one of the most commonly used type of system analysis in engineering. FEA is commonly used as a tool for analyzing boundary values. A fundamental common type of analysis is a static analysis to determine the stress and displacement profile of a geometry which would otherwise be difficult to compute with an analytical model.

FEA and multibody analysis is often kept separate in each's own respective area of expertise, however the combination of these two methods of analyses would allow each to make up for what the other program cannot perform. In the case of gear mesh analysis, a finite element model (FEM) would allow for stress analysis at various locations throughout the gear body during standard operating parameters. This indicates that stresses can captured under a dynamic force condition and/or high speed applications, a situation in which FEA is able to capture but has various limitations. MSC Adams has attempted to bridge the gap by incorporating Adams View Flex and the Adams Durability Module. However, this add-on contains significant limitations as to the level of complexity of a finite element model that can be created. View Flex is sufficient for analyzing simple bodies but lacks the level of mesh refinement that which can be created for more complex geometry. 
Under the MSC family of programs MSC Patran/Nastran exists as a FEM creator and solver. Nastran stands for NASA Structural Analysis; this program has existed since the mid 1900's originally created by NASA to aid in increasing the efficiency of aerospace vehicles. Nastran acts as the solver for FEM inputs. The current pre-mesher or meshing software that is directly compatible and integrated with Nastran is MSC Patran. Patran/Nastran currently rivals popular FEA software such as Abaqus CAE and ANSYS. The advantage of Patran/Nastran is also its compatibility with Adams, with both programs being owned under MSC. Patran/Nastran allows the generation of files that is easily interfaced with Adams models to combine the two family of engineering analysis fields, finite element analysis and multibody dynamic analysis. Other rival FEA software also have similar capabilities, however there are much more complicated procedures which are required to allow the integration.

This thesis project investigates the effect and advantage of a flexible body into a multibody dynamic analysis of a wind turbine gearbox. The vibration pattern of the planetary gear system is analyzed through Adams to distinguish certain properties that develop due to a crack or defect on a particular gear in the system. A flexible body representation of a sun gear in the planetary gear system can be analyzed for the stresses at the root of a specific gear teeth with or without defects. This type of analysis can help diagnose gearbox health and prevent critical failure and damage to a larger system as a result of one component. This research combines a FEA and multibody dynamic analysis using MSC Adams and Patran/Nastran and analyzes the vibration pattern through multiple stages of a planetary gear systems. With the help of previous research and the development of the design for a particular gearbox that models the parameters of a N90 wind turbine developed by Nordex SE in Germany, this research can be done to develop a model which has a significant and direct application. 


\subsection{LITERATURE REVIEW}

The area of study involving gear mesh systems is a very involved field and has been studied for many years. Planetary gearboxes are used in a wide variety of applications as the mode of power transfer. In this particular case, Nordex uses a two-stage planetary and single stage fixed axis gearbox to power the N90 2.5MW wind turbine [1]. Wind energy is one of the fastest growing renewable energy industry in the world [2]. Aitken outlines that wind energy is expected to reach a goal of providing $12 \%$ of the world's electricity requirement in 2020 and $20 \%$ of Europe's energy demand [6]. However, with increasing operations, wind turbines continue to suffer from component failure with high maintenance and repair costs as stated by the National Renewable Energy Laboratories (NREL) in 2012 [3]. The NREL has continuously studied the efficiency on methods to reducing or managing the high cost of drivetrain components in a wind turbine gearbox of its 20-year life expectancy design. There have been many experimental test setups by NREL to evaluate the effectiveness of the standard vibration analysis methodology.

The gear design process, outlined in Norton's Design of Machinery [5], can be done to minimize the frequency in which a single tooth can come in to contact with the other teeth on a meshing gear. Multiple studies exist on analytical models of gear dynamics, Ozguven and Houser [9] discuss the mathematical model of gear dynamics focusing on the theory and analytical methods of representing gear mesh parameters. Puigcorbe and De-Beaumont discuss the high rate of failure of gearbox design for wind applications arises from the inability to accurately predict the loads, dynamic and static, which the system experiences at any given time [4]. This leads to high engineering costs in design from over-engineering components to compensate for the high risk. The field of study of monitoring wind turbine gearbox life has been an endeavor for a period of time. Puigcorbe and De-Beaumont also point out a key feature of the standard design which has 
yet to be resolved: the effect of improper rotor support inducing high loads to the structure and gearbox [4]. The cost of maintaining such gear trains can quickly rise up to the $\$ 500,000$ range.

Alemayehu and Ekwaro-Osire discuss the life expectancy of wind turbine gearboxes to range from 3 to 7 years as opposed to the design expectancy of 20 years [8] in their study of the high speed helical gear stage design. In a survey study conducted by Ribrant and Bertling [7] an apparent trend has emerged that larger wind turbines have an increasing failure rate as opposed to smaller turbines, which exhibits the opposite trend.

To attempt to combat this costly issue, Musial W., Butterfield S., and McNiff B [13] discuss the attempt to improve wind turbine design by addressing three main points: possibility of unaccounted loading, unpredictable non-linear load transfer and individual component reliability. The resulting conclusion remains the same that new approaches to gearbox and system analysis is require to address the failure issues at all levels of the design and manufacturing process. Smolders et al. presents a reliable generic model representation of a wind turbine gearbox for reliability predictions, including all critical components [10]. They conclude that the complexity of a gearbox requires powerful analytical resources to more accurately predict the reliability.

More in-depth analysis of vibrational behavior has been performed on free vibration of planetary gears by Lin and Parker [19] using a mathematical model defining natural frequency properties. Using methods of Fast Fourier Transform, Wu, Meagher and Sommer performed research and analysis on a differential planetary system introducing backlash and teeth damage [16]. This analysis proves evident to detecting damaged components in a gear system through vibration characteristics displayed on an FFT plot with varying amplitudes. With this analysis in mind, incorporating a finite element model into a dynamic analysis will allow for stress evaluations on the required feature of interest. This is can be done through the use of MSC Adams and 
Patran/Nastran through methods of modal superposition [27]. A multibody dynamic analysis is performed at California Polytechnic State University, by Bradaric, G. [17] on dynamic behavior of a connecting rod through incorporating a flexible body into MSC Adams. This research investigates the integration between a finite element modal and multibody dynamic analysis. A conclusion was reached that the integrated systems can produce accurate stress for a dynamic body. A similar analysis was performed using MSC Adams and Abaqus FEA software by Sawatzky, Rene [11]. The focus of Sawatzky's research was to design a model to create the gearbox that would achieve the requirements of the Nordex N90 and analyze the first stage of the planetary gear system. This paper will continue further analysis of the same N90 gearbox using the combination of MSC Adams and MSC Patran/Nastran, but looking at the system as a whole in all three stages. 


\section{CHAPTER 2. - SYSTEM MODELING}

\subsection{SYSTEM MODELING - SOLIDWORKS COMPUTER AIDED DESIGN}

The system created using over all parameters provided through Nordex was modeled using the combination of 3D Computer Aided Design (CAD) Software, Solidworks, and Matlab code. The gear parameters are used to input to the Matlab code which generates a series of gear parameters required to model the gear in CAD. These parameters include the dedendum, addendum, pitch diameter, base diameter, and angle and direction of rotation for Solidworks. Along with these values, a text file is generated with coordinates of an involute profile. Solidworks image quality is turned to the maximum resolution and units are changed to metric. This involute profile is imported into Solidworks to define the critical shape of the gear tooth.

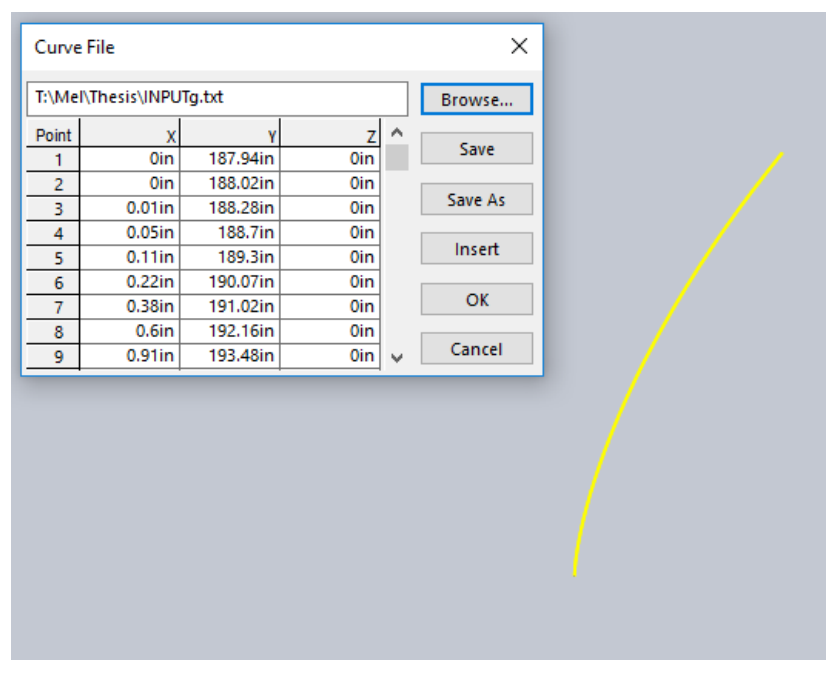

Figure 1. Gear tooth involute profile

The involute profile is rotated about the center of the gear to the specified angle of rotation and direction from the MatLab output file. The gear body and the gear tooth is extruded to thickness and then patterned to the specific number of teeth. 


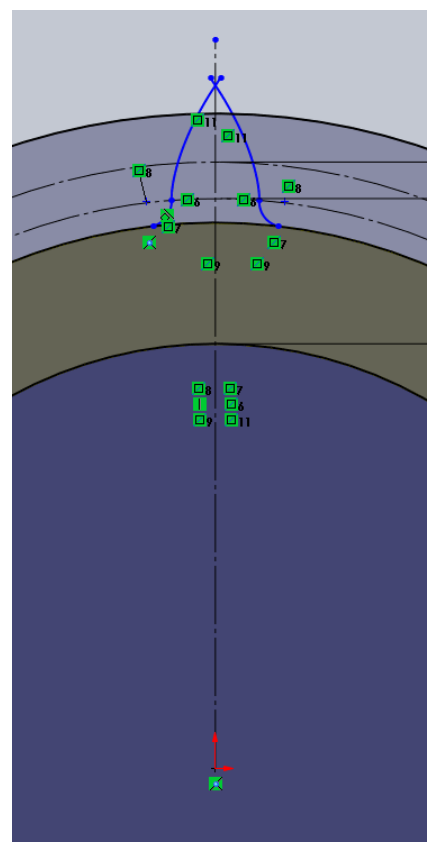

Figure 2. Gear tooth profile development in Solidworks

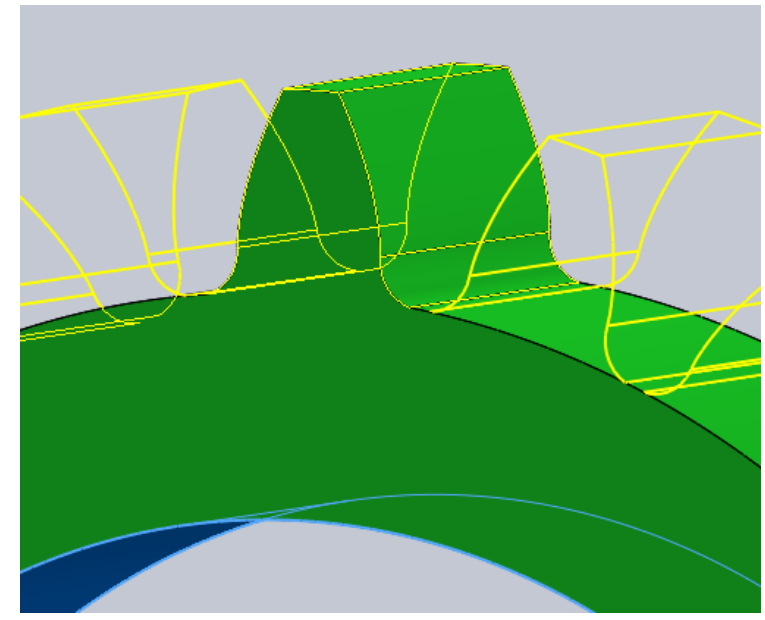

Figure 3. Gear tooth rotation to create full gear

The gear parameters for all gears are shown in Table 1 .

Table 1. Gearbox components design parameters

\begin{tabular}{|l|c|c|c|c|c|}
\hline \multirow{2}{*}{ Part } & \multirow{2}{*}{ Teeth } & Module & Pitch & Model & SolidWorks Part \\
& & {$[\mathrm{mm}]$} & Diameter & Color & \\
& & & {$[\mathrm{mm}]$} & & \\
\hline
\end{tabular}




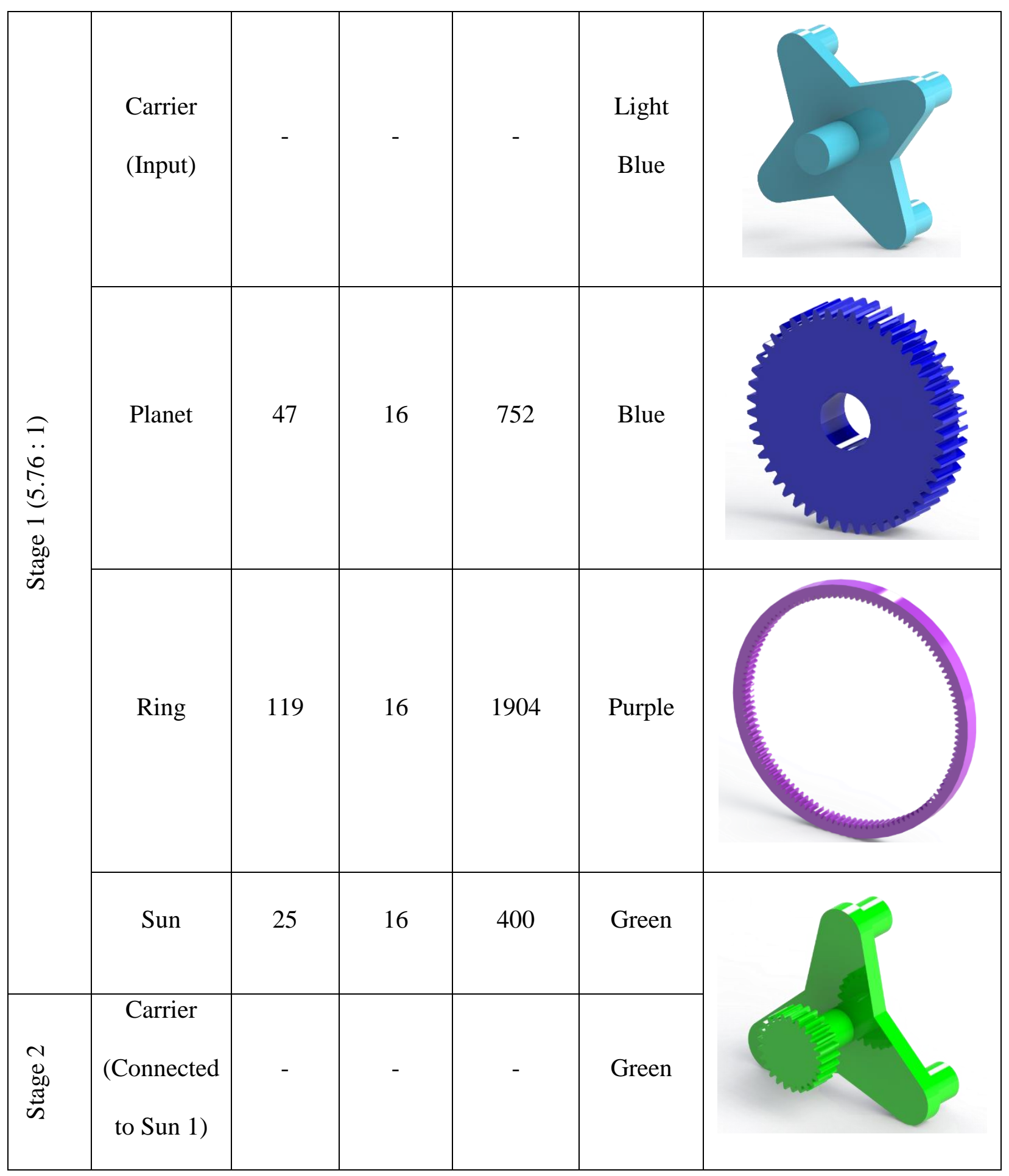




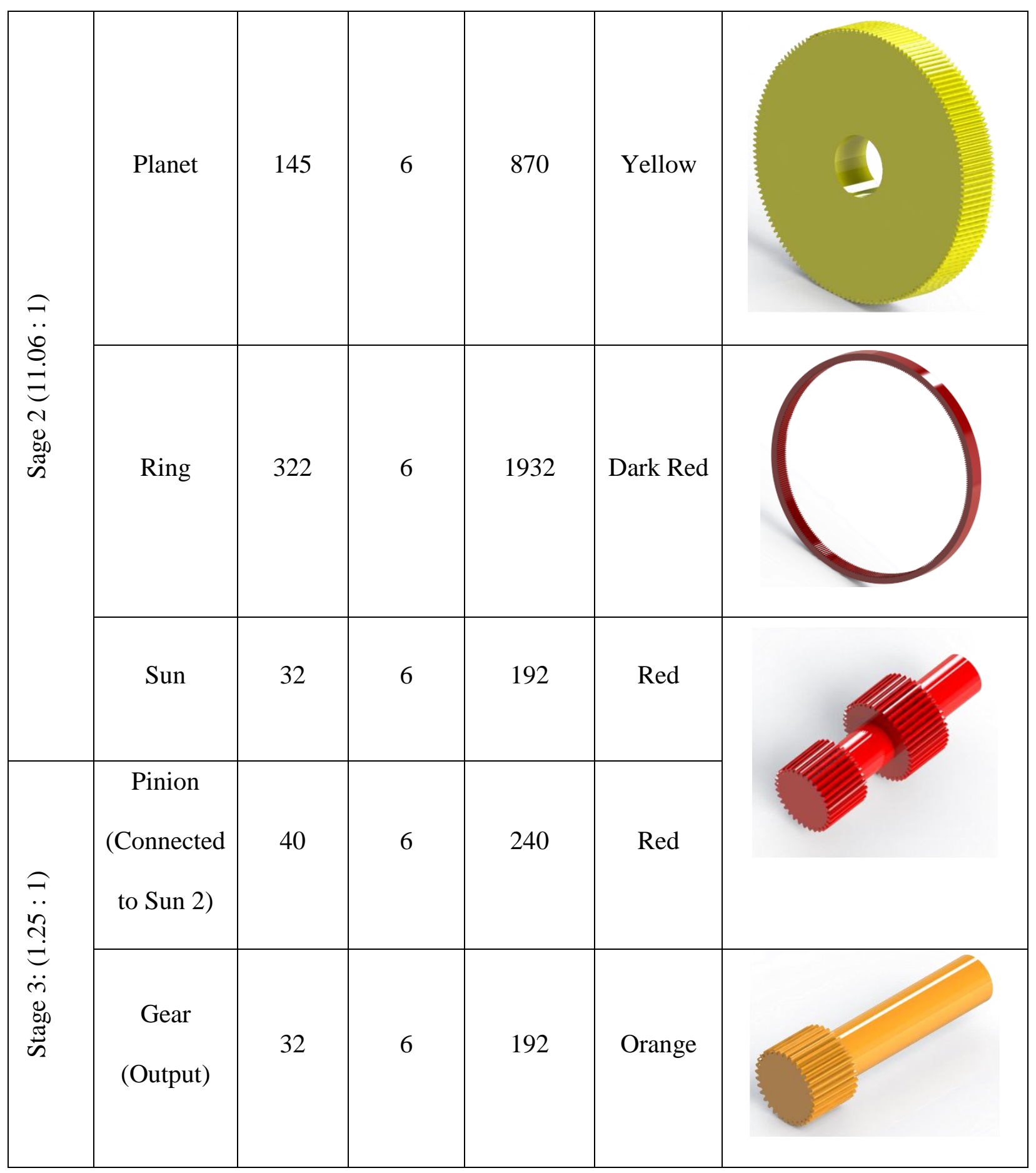




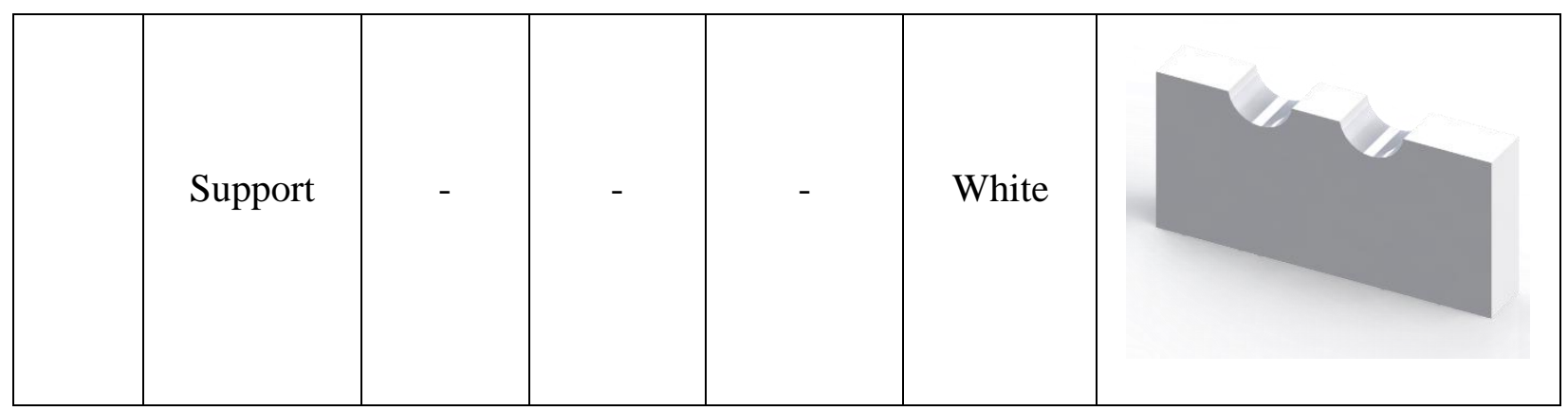

Table 1 defines full parameters for all three stages designed for the gear box. Each stage is denoted with the associated gear ratio, number of planets, teeth on each gear, module, pitch diameters, and a color indicator for the components. The parts are made with straight shafts without any keyways or shoulders features for bearing location. This is done to simplify the assembly for dynamic analysis in MSC ADAMS. The gear ratio is governed by the set overall parameters of a minimum of 77.38 to 1 ratio, this ratio does not account for power losses and represents the ratio between the input rotor speed and the generator input speed at maximum power generation. A gear design criterion was created to determine the parameters for each gear stage to achieve the necessary gear ratio as well as gears which are able to support loads introduced into the system through the input torque. These loads seen throughout the system is quite large thus resulting in gears which are non-standard size gears in the planetary system. These gears range from about $200 \mathrm{~mm}$ to $2000 \mathrm{~mm}$ in diameter and each having a thickness of $150 \mathrm{~mm}$.

Through the gear design criteria, the gears determined for each stage were created with an overall ratio of 5.76 to 1 in the Stage 1 (turbine side/input), 11.06 to 1 in Stage 2, and finally 1.25 to 1 in Stage 3. This results in an overall gear ratio of 79.6 to 1 . The pitch angle used is a standard angle at 20 degrees. With these parameters, each gear was modeled in SolidWorks using the maximum modeling resolution combined with a script program written in Matlab. The 
Matlab script is used to calculate pitch diameter, addendum, dedendum, base diameter and rotation angle. The rotation angle incorporates the $1 \%$ backlash used to model the system. This script provides a text file with coordinates for a tooth profile which is used in SolidWorks to create the gear. The $1 \%$ backlash is incorporated with the involute profile is rotated and mirrored to represent the tooth profile which is then extruded to a specified thickness then patterned. Stage 1 is designed to have gears with lower number of teeth which will see a larger input torque than the other two stages, since as the gear speed increases in the system the torque decreases proportionally with the gear ratio.

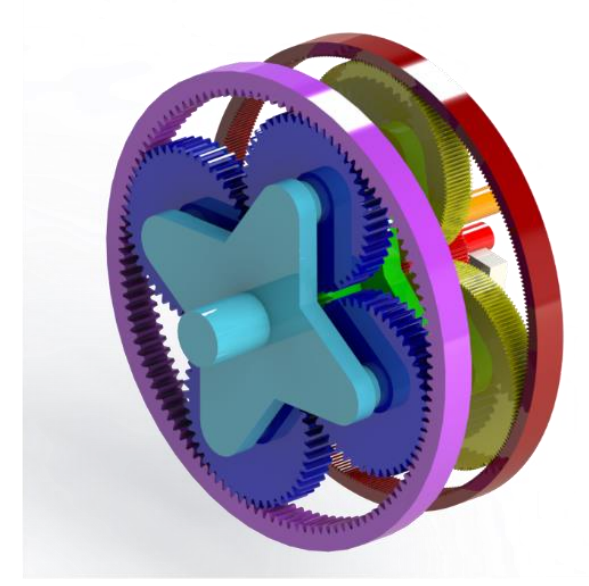

Figure 4. Full isometric Solidworks Assembly 


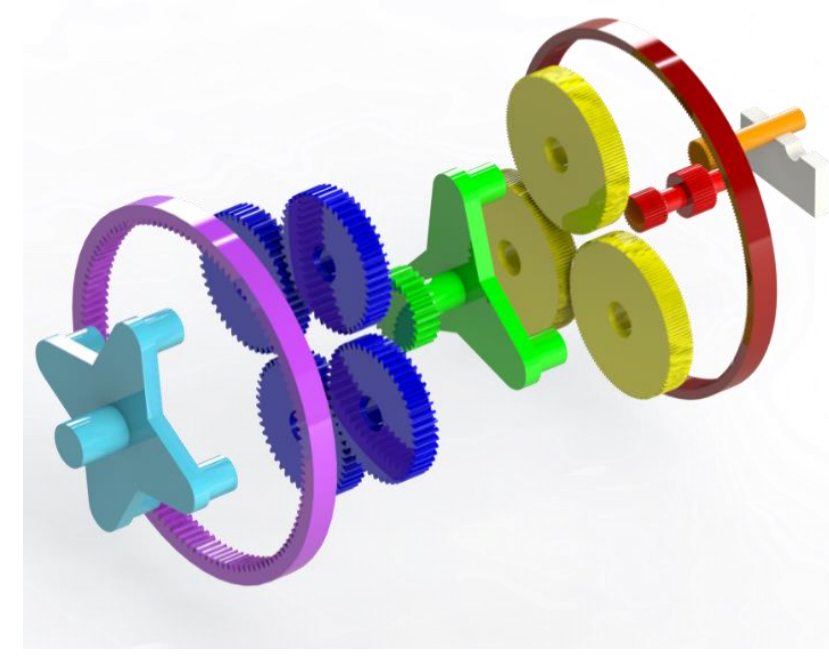

Figure 5. Assembly exploded view

With the full scaled model in mind, another simplified model was created to scale down the overall size on complexity which emanates for a full gear model with incorporated shafts. The gear models were simplified using a scaling factor on the module of the gear. This effectively causes the reduction of each gear model by the same factor as the reduction in the module. The thickness of the overall gear can also be scaled down according to the desired factor. These scaled models allowed for a much more efficient use of available computing resources for the analysis.

The new scaled model is created with a module reduction of two. The effective gear properties is reduced by the same factor. The thickness of the gear was chosen to be reduced by a factor of five. The scaling factors created must be kept in mind when creating a force/torque input into the system. The stress is ideally kept the same in order to represent the true behavior of the gear box system. The new module specifications were input into the MatLab code and the properties were generated input into Solidworks. The new model created, represents the basic gear model without an incorporated shaft. The inclusion of a shaft running through the center gear body, creates a significantly more complex model requiring more complex analysis with higher levels 
of computing power. This will be further discussed in the theory of modal analysis and flexible bodies. The system motion and constraint definitions are defined in ADAMS and will be discussed in ADAMS system modeling. The new system model is shown in Figure 6.

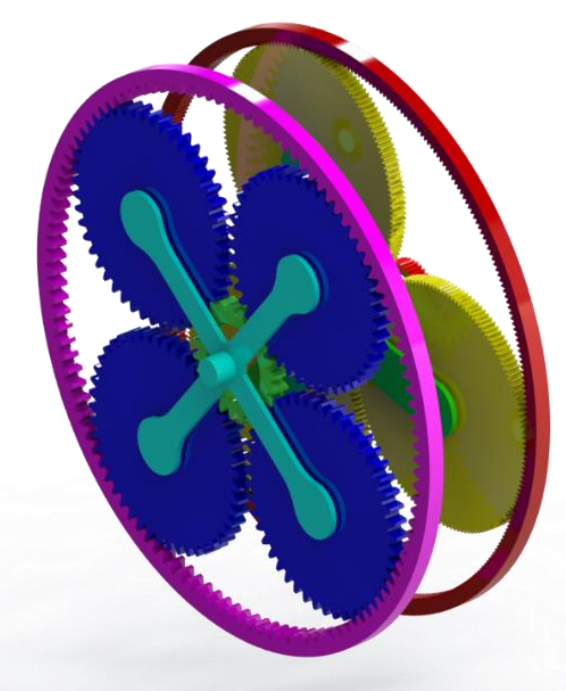

Figure 6. Scaled model assembly isometric

Figure 6 shows the full system model of the three-stage assembly. This system is broken up into three main parts; Stage 1, Stage 2, and Stage 3. Each of these stages are represented in the Solidworks model with different configurations. These different configurations allow for model to be imported into ADAMS for different types of analyses. Table 2 show the new parameter for the models

Table 2. Scaled model parameters

\begin{tabular}{|c|c|c|c|c|c|c|c|c|}
\hline & \multicolumn{3}{|c|}{ Stage 1 } & \multicolumn{3}{c|}{ Stage 2 } & \multicolumn{2}{c|}{ Stage 3 } \\
\hline & Sun & Plan & Ring & Sun & Plan & Ring & Gear & Pinion \\
\hline Module & 8 & 8 & 8 & 3 & 3 & 3 & 3 & 3 \\
\hline Thickness & 30 & 30 & 30 & 30 & 30 & 30 & 30 & 30 \\
\hline Teeth & 25 & 47 & 119 & 32 & 145 & 322 & 40 & 32 \\
\hline Pitch R & 100 & 188 & 476 & 48 & 217.5 & 483 & 60 & 48 \\
\hline Adendum R & 108 & 196 & 468.51 & 51 & 220 & 480.07 & 63 & 51 \\
\hline Dedendum R & 90 & 178 & 486 & 44.25 & 213.75 & 486.75 & 56.25 & 44.25 \\
\hline
\end{tabular}

Note: all relevant dimensions in millimeters 
Table 2 indicates the new system model parameters and the associated parts color indicators. Although, size reduction of the gears by reducing the model size through the module will not affect computational load when the part is meshed in an FEA program, the thickness reduction can effectively reduce the number elements. 


\section{CHAPTER 3. MSC PATRAN/NASTRAN FINITE ELEMENT MODEL}

In order to introduce a flexible body analysis in to ADAMS, a modal analysis on the desired body must be performed through the use of a finite element model (FEM). The FEM will be generated with the same parasolid part file out of Solidworks into ADAMS. This is done to ensure that the model remains in the same orientation and location when modeling in different software. In this study, the FEM will be created using MSC Patran and MSC Nastran. Patran is a FEM generator program which uses Nastran solver to run the analysis. A modal analysis on the gear models can be performed in order to generate a flexible body for input into ADAMS for dynamic analysis.

\subsection{MODAL ANALYSIS/SUPERPOSITION}

Modal superposition is the combination of linear, small deformation modes on a body. Through this theory, a larger body can be defined as having different modes, deformation shapes, of increasing order which are then superimposed. This allows for the representation of models with a variety of degrees of freedom with a series of modal degrees of freedom. The overall vibration characteristics of a body can be represented with the combined superposition of multiple mode shapes. An example of three different mode shapes of a beam is represented in Figure 7. 

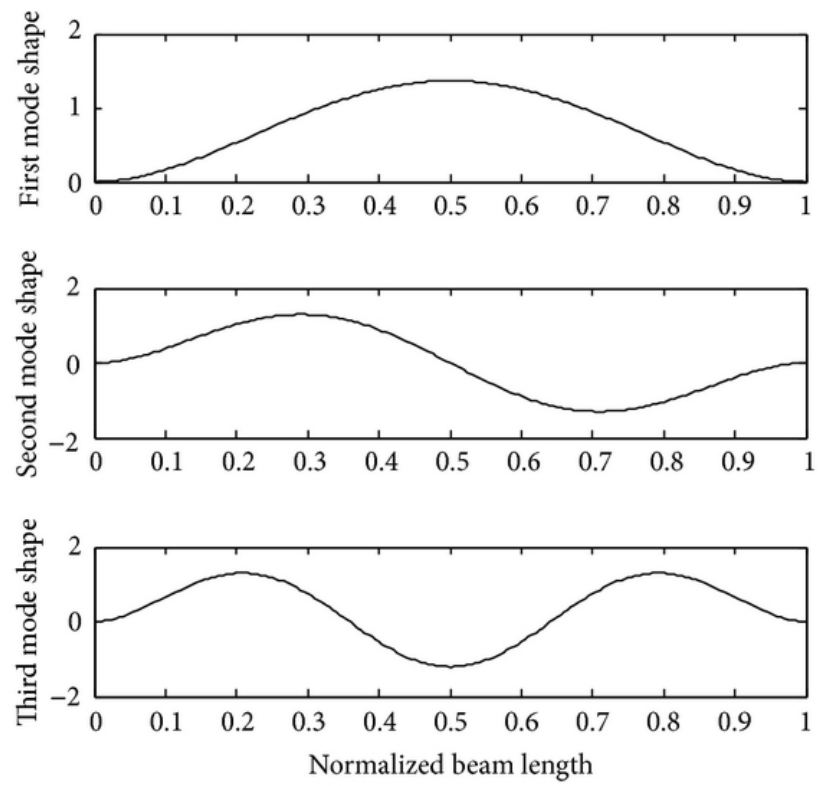

Figure 7. First three bending modes for a beam with Fixed-Fixed boundary conditions [25] The mode shape superposition approximation of the vibrational behavior of a model follows a mathematical summation of the different mode shapes and their associated amplitudes.

$$
v(x, t)=\sum_{i=1}^{M} \psi_{i}(x) * q_{i}(t)
$$

Equation 1 shown is representative of the displacement of a body with respect to location, $x$, and time, $t$, can be represented by the summation from the first mode to the $\mathrm{M}^{\text {th }}$ mode of the corresponding mode shape, psi $(\psi)$, scaled by $q$ which is the amplitude of the vibration mode at a specific time.

Since, normalizing the amplitude, $q$, ranging from 0 to 1 , the mode shape can be analyzed at the time which generates the maximum amplitude of each mode resulting in:

$$
v(x)=\sum_{i=1}^{M} \psi_{i}(x) * q_{i}
$$

Equation (2) shows the deformation body depending on the sum of each consecutive mode shape scaled but an amplitude factor, $q$, and position along the body, $x$. 
The method of FEA incorporation into Adams is discussed in MSC Adams - Theory of Flexible Bodies [27]. The original method utilized in Adams FEA was a method called the Guyan reduction method. With this method the software, automatically reduces and condenses the degrees of freedom (DOF) to reduce their number and computational intensity. This method attempted to match natural frequencies and geometric properties using similar elements to those created for the standard dynamic bodies. This produced undesirable results sometimes being unable to match the total mass with the newly created lumped mass matrix.

The solution to the issues produced by earlier models of Adams Flex FEA modeling was the development of the Craig-Bampton Method of Component Mode Synthesis (CMS). The CMS method is an adaptation to Adams of the modern modal superposition theory. Within CMS, specified DOF's can be isolated from the modal superposition, this treats these nodes as "boundary DOF" [27]. The static mode shapes are obtained by subjecting the boundary DOF to "unit displacements while holding all other boundary DOF fixed" [27]. This generates the constraint mode shapes, examples of these mode shapes are shown in Figure 8.
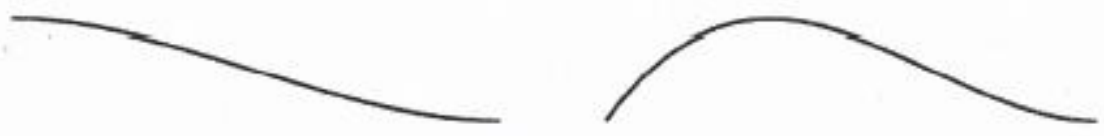

Figure 8. Simple beam with two constraint modes

Figure 8 shows two constraint modes for the left end of a beam with attachments at each end.

The left mode shape resembles a unit translation mode and the right a unit rotation mode.

To develop mode shapes suitable for dynamic analysis, the mode shapes are orthonormalized by first solving for the eigenvectors to create a basis matrix following the relationship:

$$
\hat{K} q=\lambda \hat{M} q
$$




$$
u=\sum_{i=1}^{M} \phi_{i} q_{i}=\sum_{i=1}^{M} \phi_{i} N q^{*}=\sum_{i=1}^{M} \phi_{i}{ }^{*} q^{*}
$$

The process of orthonomalizing creates a resulting set of mode shapes which are orthogonal called Craig-Bampton modes [26], [27]. These modes are concluded to sufficiently address the deficiencies of other models to represent a flexible body.

The integration between Patran/Nastran and ADAMS is created through a Modal Neutral File (mnf). The mnf contains the information of the modal analysis performed by Nastran on the FEM model from Patran. This file contains the flexible body with the associated properties including but not limited to mass, geometry, element divisions, Young's modulus, poisons ratio, density and fixed node locations. The fix node locations act as fix point where the bearings would generally be located, in the case on either ends of the gear shaft. The ability to automatically generate a mnf is simplified through using Patran/Nastran for direct interface with ADAMS to be analyzed using the Durability module, the programs are created by the same software company under the name MSC and are made so that they can complement each other. Other finite element software (FEA), such as Abaqus, are capable of generating a mnf as well, however there are many features to that method which complicates the interface into ADAMS. Due to the limitation of other FEA softwares. Patran/Nastran is used for this analysis. A Patran/Adams Interface Tutorial document is attached as Appendix 1.

\subsection{PRELIMINARY MODAL ANALYSIS}

A modal analysis was performed on the sun gear to determine and create a visual representation of the different modes the sun gear model undergoes. The modes seen in the modal analysis simulate the modes that Patran/Nastran will use to create a combined modal for import to Adams 
to allow for a flexible body dynamic analysis. Figures 9 and 1 shows two of the vibrational/deformation modes the model undergoes.

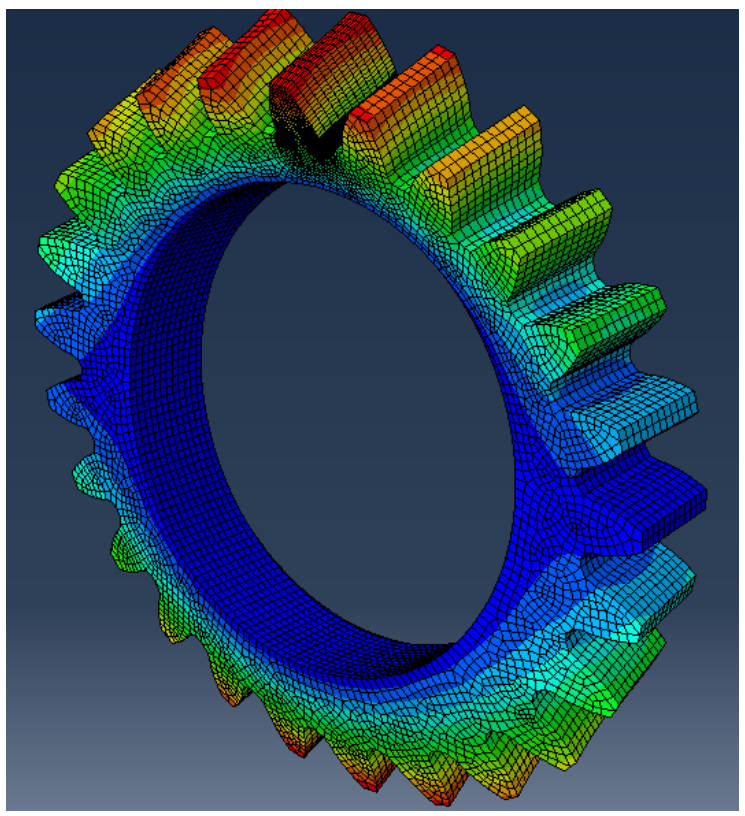

Figure 9. First vibration mode for the first stage sun gear

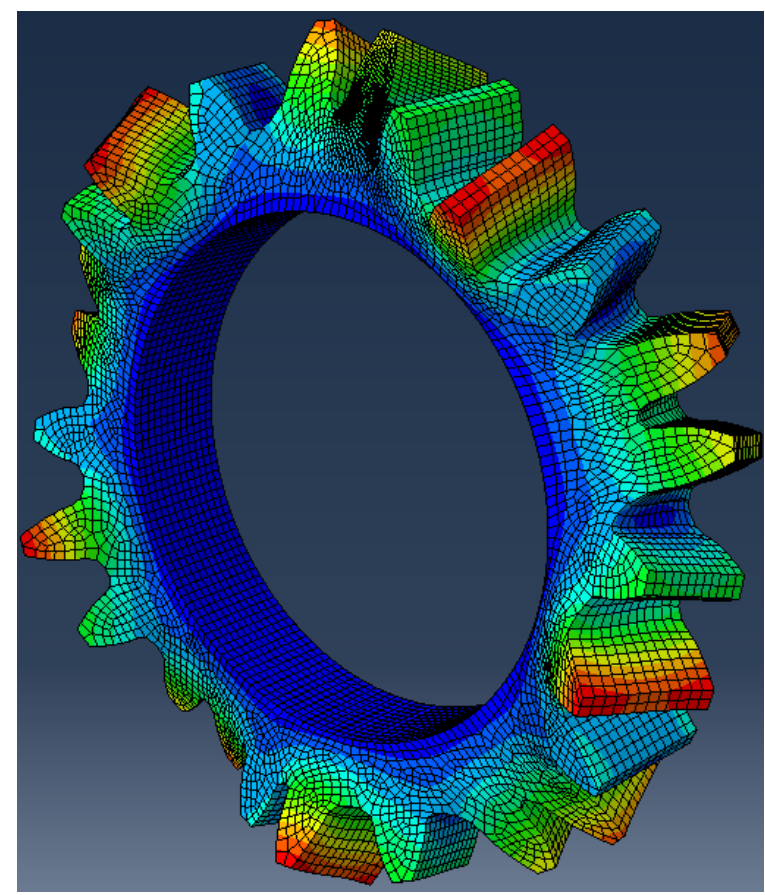

Figure 10. Eleventh vibration mode for the first stage sun gear 
The series of modes seen in this analysis gives a general visualization of how the gear teeth modes behave. Which allows for a better understanding of the theory behind the creation of a modal analysis in Patran.

\subsection{SOLIDOWORKS MODEL PREPARATIONS}

The gear mesh under analysis is the fix axis gear mesh in Stage 3. The model developed for mesh study is created in Solidworks with certain specific features so as to accommodate to Patran when meshing the model to generate elements and nodes. A part model is shown in Figure 11.

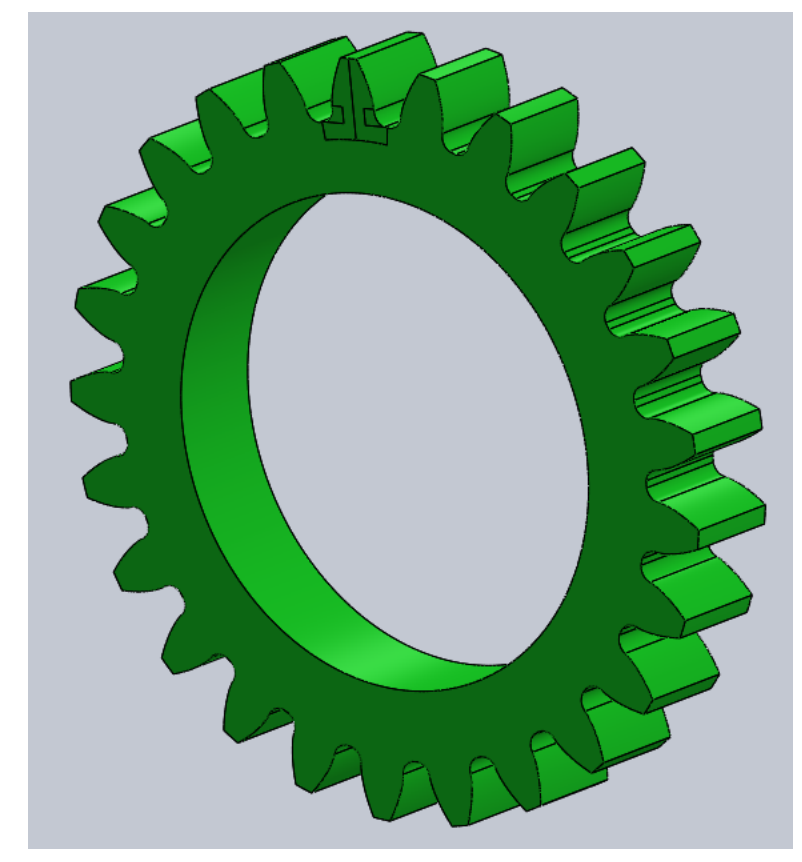

Figure 11. Solidowrks model partitioned tooth 


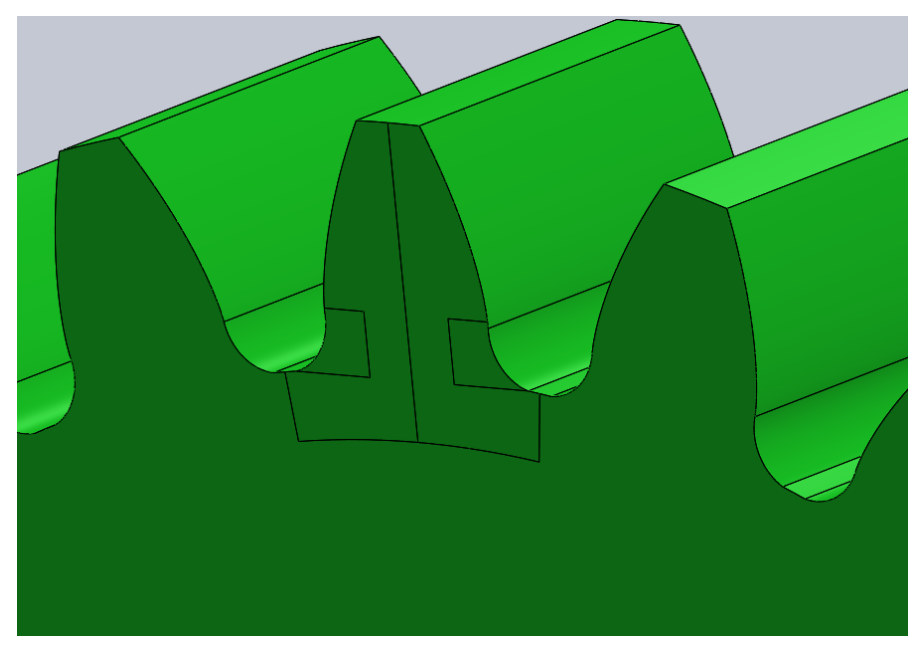

Figure 12. Close-up of partitioned tooth

The split faced features developed in Solidworks in Figure 11 is done so that, in Patran, specific seed sizes can be created on the body. A varying seed size is created to reduce the computational load on the computer hardware. A fine mesh on the teeth and at the root will result in a fine mesh on the entire part. This is very computationally intensive, generating result files from Patran that are very large in size. A significantly more power computer is required in order to run a fine mesh on the full part. The mesh seed creates a biased mesh with a fine mesh on the teeth and teeth root and a courser mesh on the main body of the gear. This significantly reduces the number of nodes and elements in the model which is directly related to the computational power required to analyze the model.

By using Solidworks to split the faces on the model, this creates a prepartitioned part going in to Patran and it also allows the selection of edges which would otherwise not be there in order to create this varying mesh. To have a proper mesh for the desired location on the root of the tooth in question to obtain accurate results, the partitions made allow for different element sizes at different location. 


\subsection{MESH ANALYSIS}

The mesh development process is done to ensure that the mesh is sufficient to provide a proper representation of the stress at the required location. This results from the process of developing a finite element analysis. When developing a representation of a solid model with the combination of multiple elements, a minimum element size is required depending on the geometry of the model in order to capture the full representation of the behavior of a body under a specified loading condition. Each element is defined by a set of algebraic equations of a specified order, generally either a linear or quadratic. An increase in the order of the element increases the complexity of the model as well as the required computational resources. The underlying efforts of a finite element model is to develop a model with a series of elements that is sufficient to capture the behavior of the model for the specific analysis and no more. Over complicating a finite element model is inefficient in terms of the resources required to perform a certain analysis which is represented in computational time.

In order to determine the minimally sufficient element parameters to create the finite element model, a mesh convergence analysis must be performed to show that the mesh does indeed provide the necessary information to capture the required behavior of the model. Figure 13 shows the mesh analysis performed on a slice of a gear looking at the stress at the root of a single tooth. These stresses for the required convergence of the mesh is depicted in Table 3 and Figures 13 and 14. 


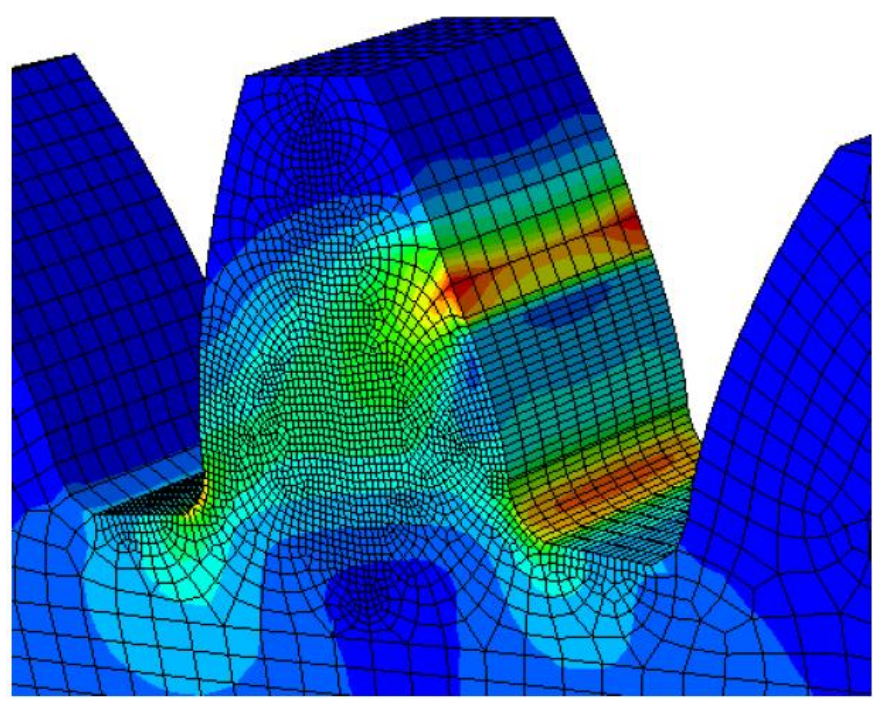

Figure 13. Meshed tooth for convergence study

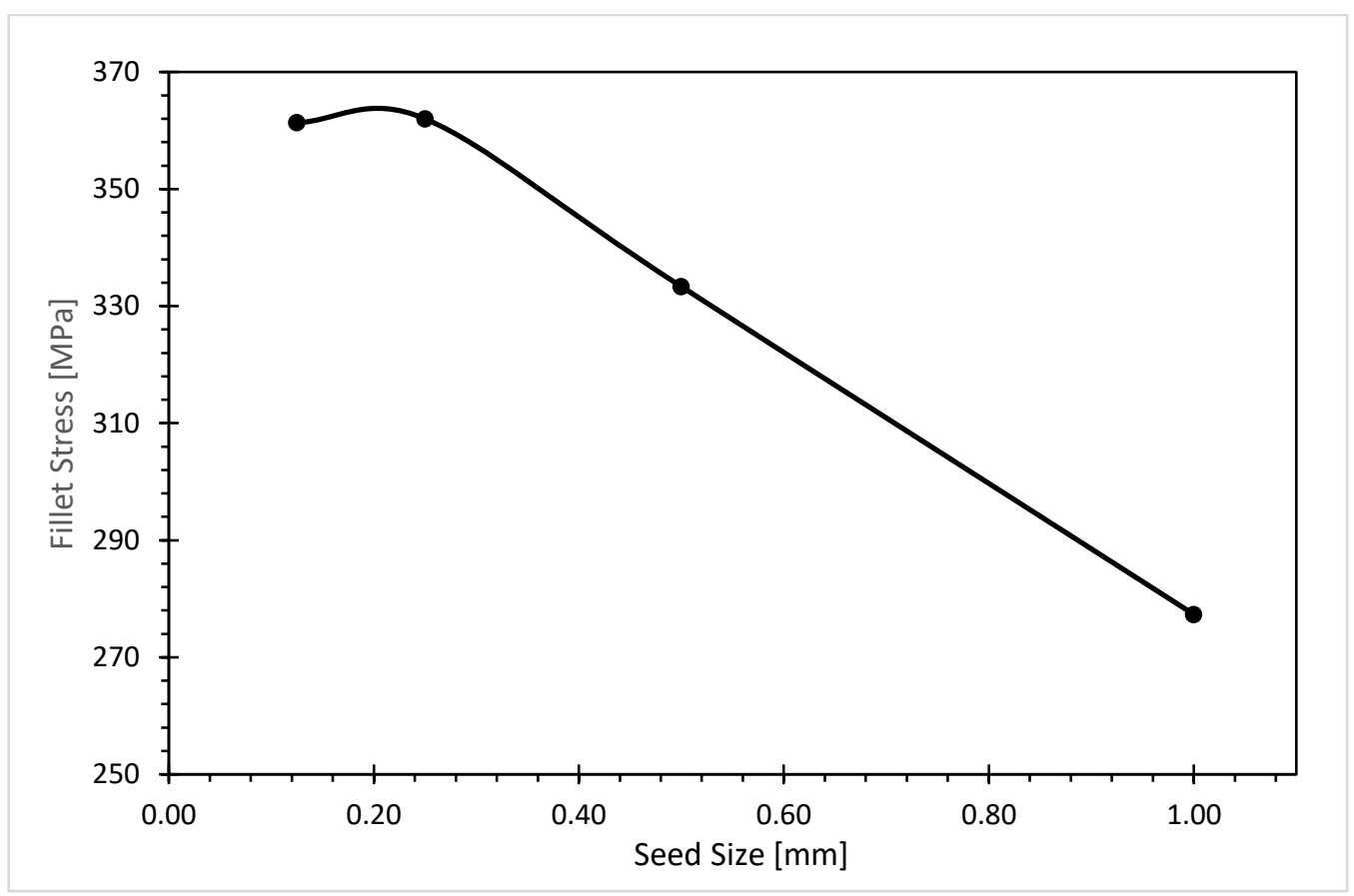

Figure 14. Convergence Study

Table 3. Mesh Convergence Study for Stress with Varying Seed Size

\begin{tabular}{|c|c|c|}
\hline Seed Size & \multicolumn{2}{|c|}{ Fillet Stress } \\
\hline$[\mathrm{mm}]$ & [MPa] & \%Change \\
\hline 1.00 & 277 & \\
\hline
\end{tabular}




$\begin{array}{lll}0.50 & 333 & 20.19 \\ 0.25 & 362 & 8.60 \\ 0.125 & 361 & 0.18\end{array}$

Figure 13 shows the sufficient mesh created for an appropriate convergence to capture the stress behavior on the root of the gear. Although, this test must be performed with measured values at the desired location, an initial goal for the fine mesh at the desired location is to have at least 6 elements at the location of analysis [Source Doug MSC]. This general procedure must always be verified, however it can be used as a starting point for defining element sizes.

A mesh convergence study will define a mesh that has converged once the stress variation is less than $1 \%$ when reducing the element size by half. Table 3 shows the development of the mesh and the changes in stress as the mesh refinement is increased by reducing element sizes. The convergence study plot is shown in Figure 14 for root stress as a function of element seed size at the specified location of interest. In Table 3 the change between $0.25 \mathrm{~mm}$ edge seed sizes and $0.125 \mathrm{~mm}$ shows slight variation of stress of $0.18 \%$. The mesh can be defined as converged at with this element size between $0.25 \mathrm{~mm}$ and $0.125 \mathrm{~mm}$ seed size, and $0.25 \mathrm{~mm}$ seed size on this model can be used. With a reduced model size, the same scale factor can be incorporated for the mesh properties. The mesh convergence analysis is primarily significant to the location in which the stress is to be analyzed. In this case the root stress of the gear in question.

\subsection{STATIC ANALYSIS}

To verify the appropriate range of stress for the validation of the finite element analysis, as static cantilever beam analysis is performed to approximately predict the behavior of the stress at the root of the tooth. This analysis is performed as a cantilever beam stress approximation 


$$
\begin{gathered}
\sigma=\frac{M * y}{I} \\
\sigma=\frac{F_{t} * r * \frac{h}{2}}{\frac{1}{12} * b * h^{3}}
\end{gathered}
$$

In equation $\mathrm{EQ}(\#)$, the variable $F_{t}$ represents the tangential force applied to the gear. The variable $r$ represents the simulated moment arm. $h$ represents the root thickness of the gear and $b$ is the face width of the gear.

Table 4. FEA model comparison with cantilever beam

\begin{tabular}{ccc} 
Analysis & $\begin{array}{c}\text { Stress } \\
{[\mathrm{MPa}]}\end{array}$ & \% Diff \\
& & \\
\hline Abaqus & 362 & 28 \\
Beam & 282 &
\end{tabular}

A 30\% difference as shown in Table 4 shows a respectable range for a beam representation of a more complex geometry of the gear tooth. It must be noted that this stress calculation method is quite simplified and is used to approximate the range at which the stress is expected to be. For true estimates to gear bending stress and contact stress the AGMA standards must be applied.

\subsection{PATRAN FINITE ELEMENT MODEL}

The prepartitioned Solidworks model is imported into MSC Patran as a parasolid model. This model is representative of the model to be used in ADAMS for dynamic analysis. This ensures the proper orientation of the model when importing the .mnf file between programs. The part to be created as a flexible body is isolated in Patran, this Stage 1 sun gear is shown in Figure 15. 


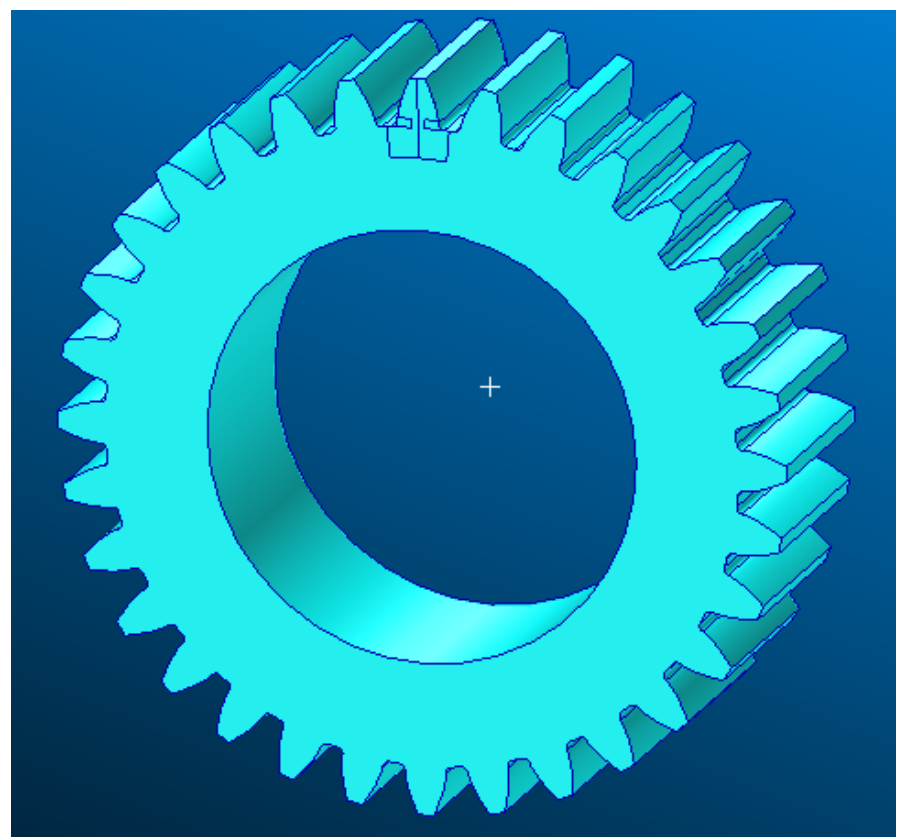

Figure 15. Patran geometry import model

Figure 15 shows the tooth prepared for analysis inside of Patran. This is the same tooth in which a defect will be later applied to compare final results after the dynamic multibody simulation inside of ADAMS. A close up mesh location is shown in Figure 16.

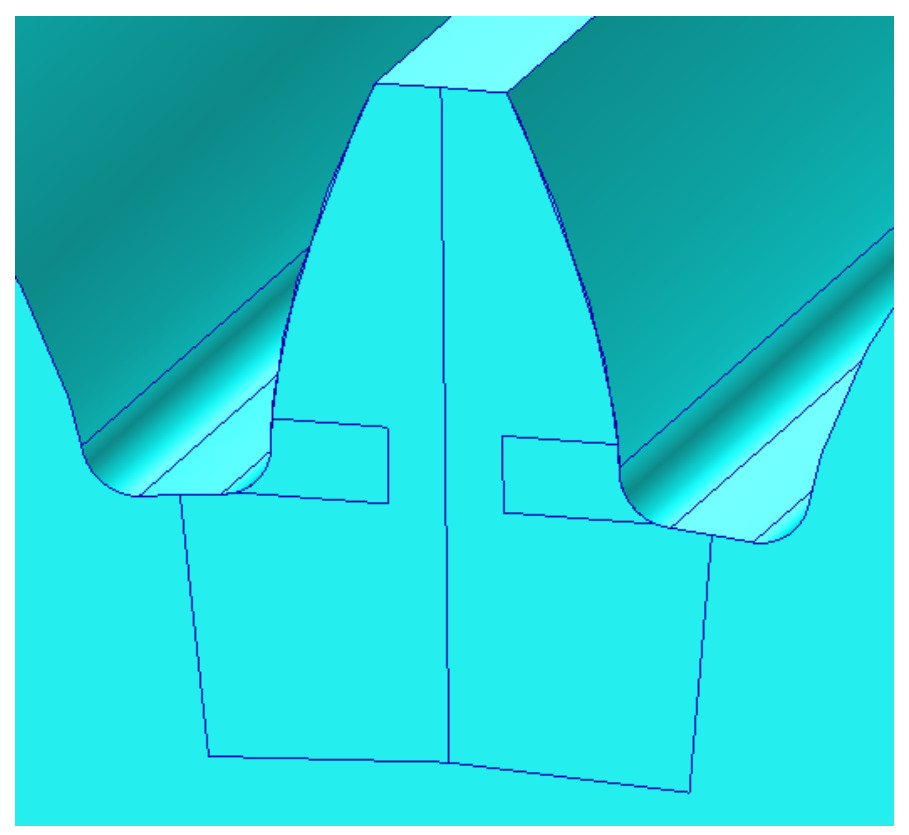

Figure 16. Patran close-up partition 
Figure 16 shows the primary location for the varying seed size assignments on the gear tooth. The mesh parameters are show in Table 5 for each surface.

Table 5. Mesh Properties

\begin{tabular}{lccc} 
Element Type & Element Shape & Seed Size \\
& Function & Location & {$[\mathrm{mm}]$} \\
& & Root & 0.125 \\
Quadrilateral & Linear & Tooth Body & 0.5 \\
(2 Dimensional) & $($ Quad4) & Gear Body & 4 \\
& & Thickness & 5 \\
\hline \hline
\end{tabular}

The mesh is the symmetric for both sides of the gear tooth. The mesh created at the root for stress evaluation is assigned as $0.125 \mathrm{~mm}$. This is in accordance with the mesh convergence study performed for the same gear model at twice the size, thus a seed size of half of $0.25 \mathrm{~mm}$ was used. At the tooth and the surrounding area, a seed size of $0.5 \mathrm{~mm}$ was used and the overall gear was meshed using a $4.00 \mathrm{~mm}$ seed size. The critical stress analysis point is concentrated at the root of the gear tooth, and the relatively fine mesh is used for the tooth and the surrounding area. The rest of the gear body and other teeth are meshed with a coarse mesh to reduce the number of elements in the body for computational purposes. The gear tooth, itself does not require a mesh as fine as that on the fillet at the root of the gear for accurate results. Figure 17 and Figure 18 shows the meshed gear body. 


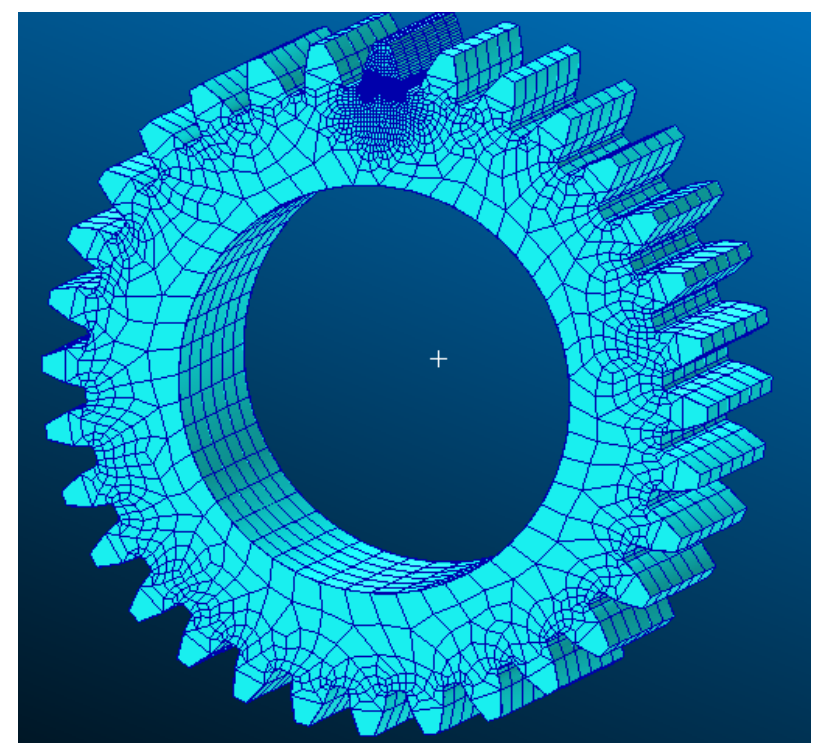

Figure 17. Meshed Patran part

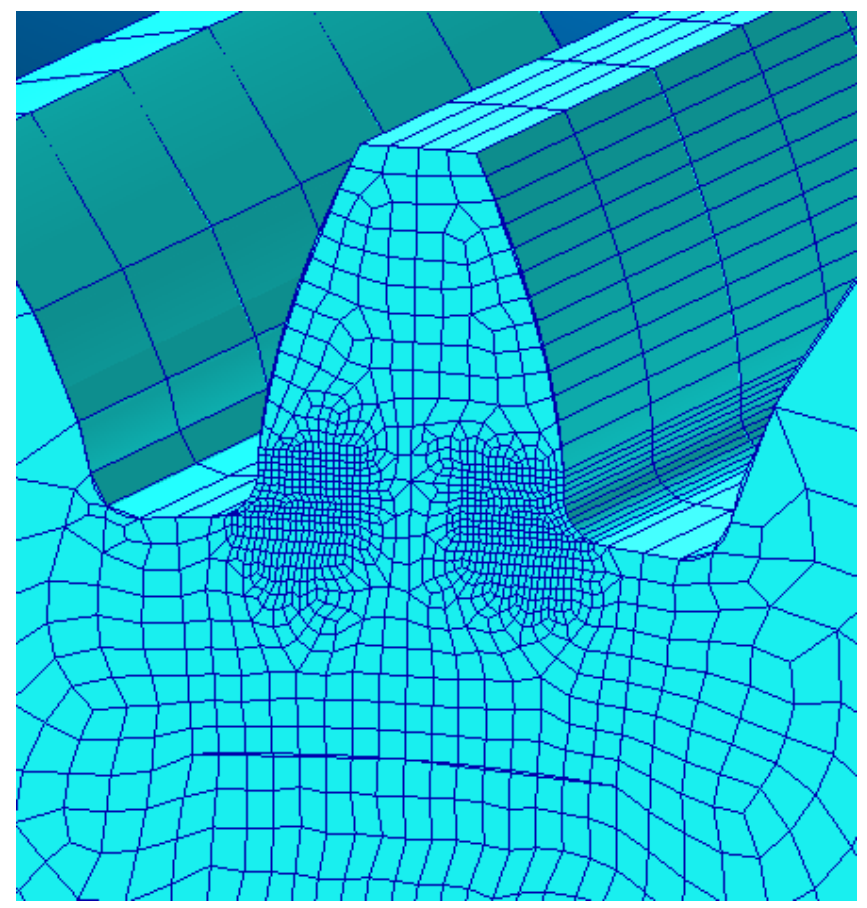

Figure 18. Mesh close-up Patran

The mesh was created as a two-dimensional surface mesh on the face of the gear and then extruded the thickness of the part. This method allows for a consistent mesh through the 
thickness as opposed to meshing the whole solid instead with three dimensional elements which produces undesirable results.

With a varying surface mesh on different areas of the gear face, there are nodes which are created at the same locations on the same edge. These nodes will results in errors when attempting to run the modal analysis. A process called 'equivalence' is used to eliminate these nodes and associate the different meshes together along a common edge. Equivalence is performed whenever a new mesh is created or elements are extruded next to other meshed surfaces but still remain part of the original body. When the surface mesh is swept/extruded through the thickness of the part with $5 \mathrm{~mm}$ thick elements with 6 elements, Patran creates a separate set of elements which are independent from the original solid. A material assignment was created for each element and not the overall solid. The old solid is left as is without any defined properties. Although the solid element has no defined properties, the analysis was still performed on the remaining elements, this does not affect the solution; the undefined element is remains un-"translated" by Patran/Nastran and can remain hidden. The material properties entered into Patran are those represented in Table 6.

Table 6. Material Properties

\begin{tabular}{lcc}
\multicolumn{1}{l}{ Material } & AISI 4820 Steel / 18CrNiMo 7-6 / UNS G48200 \\
\hline Mass Density & $7.77^{*} 10^{\wedge}-6$ & $\mathrm{~kg} / \mathrm{mm}^{3}$ \\
Stiffness & 210 & $\mathrm{GPa}$ \\
Poison's Ratio & 0.29 & \\
Yield Strength & 685 & $\mathrm{MPa}$ \\
Ultimate Strength & $840-1200$ & $\mathrm{MPa}$ \\
\hline
\end{tabular}


The constraint must be defined for the gear body so that the modal analysis can be performed. The constraint location is positioned at the center of the gear body. A node is created to constrain the internal face of the gear to the center of the geometry. The reference nodes created at the center of the bore are connect with perfectly rigid body elements, this is defined in Patran as an RBE2. The central node is defined with a set of degrees of freedom. The software uses this node as the fixed boundary condition for analysis. This has a similar effect of defining a fixed constraint (Abaqus) to the internal face of the gear, where all translational and rotation degrees of freedom are constrained. Figure 19 illustrates this node relationship.

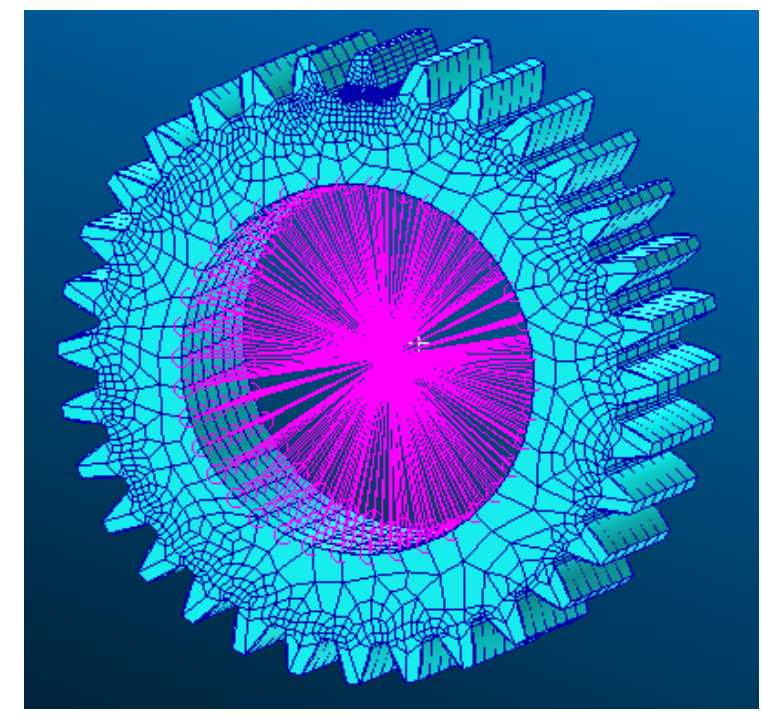

Figure 19. RBE2 Spider constraint of gear body

The RBE2 created is represented by the pink lines in Figure 19. These lines connect to each internal node on the gear surface elements.

The normal mode analysis is performed by Nastran once Patran has completed its initial translation of the elements. The modal analysis set up is created with 40 normal modes for the gear body. Patran also includes 6 modes for the translation and rotation of the reference node which creates a total of 46 modes of the model. This analysis generates the .mnf output file from Nastran for input into ADAMS. 


\section{CHAPTER 4. MSC ADAMS DYNAMIC MODEL}

Once the system is complete in Solidworks the file is converted to a parasolid for input into MSC ADAMS, the file used is the same parasolid input for Patran. In ADAMS units are set appropriately as millimeters, newton, kilograms and seconds (MMGS). The parasolid is then imported into ADAMS.

The Adams assembly is located in the same place as where the origin is defined in the Solidworks model. This assembly is representative of the full size system with incorporated gear shafts and full gear bodies. The color identification of the parts remains the same from those imposed in the Solidworks model once imported into ADAMS. This allows references back to Table 1 for component parameters. Once assembly has been imported the density/material of each part has to be defined. The martial properties for the material selected for the components are defined in Table 6 under the finite element model section.

AISI 4820 Steel the mass density for Table 6 is enter for all components in the assembly. Note the density must be defined in the specified working units, in this case material mass density is converted to $\mathrm{kg} / \mathrm{mm}^{\wedge} 3$.

\subsection{JOINTS AND CONSTRAINTS}

In reality, a wind turbine gear box will include the housing assembly, shaft splines, shaft keys/keyways, as well as bearings in to locate the parts in the proper location. These additional features will add higher order dynamics to the system due to bearing stiffness and rotor imbalance due to non-symmetric geometry in the system. There are a variety of methods to model, analyze or compensate for such system dynamics, however for the purposes of this 
system, pure model is created with simple revolute joints connecting each part relative to each other. The revolute joint for the full system is defined in Table 7.

Table 7. Joint properties in Adams

\begin{tabular}{|c|c|c|c|c|}
\hline Stage & Type & Body 1 & Body 2 & $\begin{array}{c}\text { Location } \\
\text { (Centered) }\end{array}$ \\
\hline \multirow{3}{*}{3} & Lock & Support & Ground & Ground \\
\hline & \multirow{2}{*}{ Revolute } & Input (gear) & Support & Ground \\
\hline & & Output (pinion) & Support & Ground \\
\hline \multirow{5}{*}{2} & Lock & Ring 2 & Ground & Ground \\
\hline & \multirow{4}{*}{ Revolute } & Carrier 2 & Ring 2 & Ring 2 center \\
\hline & & Planet 2.1 & Carrier 2 & $\begin{array}{c}\text { Carrier } 2 \text { Pin } \\
\text { axis }\end{array}$ \\
\hline & & Planet 2.2 & Carrier 2 & $\begin{array}{l}\text { Carrier } 2 \text { Pin } \\
\text { axis }\end{array}$ \\
\hline & & Planet 2.3 & Carrier 2 & $\begin{array}{l}\text { Carrier } 2 \text { Pin } \\
\text { axis }\end{array}$ \\
\hline \multirow{6}{*}{1} & Lock & Ring 1 & Ground & Ground \\
\hline & \multirow{5}{*}{ Revolute } & Carrier 1 & Sun 1 & Ring 1 center \\
\hline & & Planet 1.1 & Carrier 1 & $\begin{array}{l}\text { Carrier 1 Pin } \\
\text { axis }\end{array}$ \\
\hline & & Planet 1.2 & Carrier 1 & $\begin{array}{c}\text { Carrier 1 Pin } \\
\text { axis }\end{array}$ \\
\hline & & Planet 1.3 & Carrier 1 & $\begin{array}{l}\text { Carrier 1 Pin } \\
\text { axis }\end{array}$ \\
\hline & & Planet 1.4 & Carrier 1 & $\begin{array}{c}\text { Carrier 1 Pin } \\
\text { axis }\end{array}$ \\
\hline
\end{tabular}

Revolute joint dynamics is simplified to a single rotational degree of freedom in a rigid joint. This does now allow for any flexibility or compliance between the gears and the shaft in which they are joined to. The location of the joints represent shaft and bearing location between the gear body and its center shaft. Without bearing dynamics the gear only rotates about the central axis of the shaft and has no out of plane movement. It is determined that under standard operations, a uniform lateral load to the gear teeth throughout a cycle produces negligible out of plane bending moments. Since the carrier shaft is realistically supported on both ends on either side of the gear unlike the system model created, the load transfer to Stage 2 from Stage 1 
through the carrier of the first stage driving the planets on the second stage also produces no out of plane loads or bending moments.

Also seen in Table 7, the lock joints created between the ring gears and the final stage support. The lock joint on the support is used to indicate a revolute joint for the Stage 3 fix axis gears which would been realistically supported by the housing. The ring gear is designed to be fixed with the chosen gear ratio between the stages.

\subsection{CONTACT AND INTERATIONS}

The interactions between the gear teeth is modeled in ADAMS as a contact force between each body. The contact force represents the gear teeth mesh and must be created between each meshing or contacting body in the system. The body contact forces are shown in Table 8 .

Table 8. Solid body contacts in Adams

\begin{tabular}{|c|c|c|}
\hline & Body 1 & Body 2 \\
\hline Stage 3 & Output Pinion & Sun 2 \\
\hline \multirow{6}{*}{ Stage 2} & \multirow{3}{*}{ Sun 2} & Planet 2.1 \\
\hline & & Planet 2.2 \\
\hline & & Planet 2.3 \\
\hline & \multirow{3}{*}{ Ring 2} & Planet 2.1 \\
\hline & & Planet 2.2 \\
\hline & & Planet 2.3 \\
\hline \multirow{8}{*}{ Stage 1} & \multirow{4}{*}{ Sun 1} & Planet 1.1 \\
\hline & & Planet 1.2 \\
\hline & & Planet 1.3 \\
\hline & & Planet 1.4 \\
\hline & \multirow{4}{*}{ Ring 1} & Planet 1.1 \\
\hline & & Planet 1.2 \\
\hline & & Planet 1.3 \\
\hline & & Planet 1.4 \\
\hline
\end{tabular}

Table 8 shows the contact location between each meshing body in the system. These contact forces are modeled as an Impact type contact. There are 4 different criteria that must be defined for each contact stress: force exponent, damping, penetration depth, and contact stiffness. 


\subsection{FORCE EXPONENT}

The force exponent, $e$, describe the elasticity of the contact. This represents the non-linear function that models the impact contact parameter [15]. The value of $e$ is a material property. Stiffer material or hard metals such as steel will have an $e$ value of approximately 2.2. For softer, more malleable materials/metals as aluminum, $e$ is approximately 1.5. For soft materials like rubbers or certain polymers, $e$ is approximately 1.1. It is recommended to that $e>1$, a value less than one can cause discontinuities during the impact.

\subsection{DAMPING}

The damping coefficient is determined to have a maximum value of $1 \%$ of the stiffness. This is a non-physical property. Note that experienced individuals with this impact criteria believe that $1 \%$ is quite large and should be decreased. This general parameter is specified by MSC's characterization of contact impact modeling [21].

\subsection{PENETRATION DEPTH}

Penetration depth defines the behavior of the contact where damping varies between zero and the maximum damping coefficient. This value has a positive relationship to the damping constant. At lower penetration there is lower damping from zero until the maximum penetration which is associated with maximum damping constant. The recommended penetration depth is generally $0.01 \mathrm{~mm}$

\subsection{CONTACT STIFFNESS}

Contact stiffness depends on the geometry of the contacting features, in this case the gear teeth not just simply the material. The contact stiffness between the two gear faces can be modeled as a Hertzian contact between two cylinders. The contact stiffness varies across the gear mesh as the geometry/curvature of the face changes. The contact stiffness calculations require multiple 
assumptions and approximation. It must first be noted that this research topic does not dive into true dynamic model of the interaction between gear teeth. The topic of contact stiffness between gear teeth of varying geometry through the contact patch is a complete research topic in and of itself.

The Hertzian Contact model accounts for the elastic deformation in the two geometries in contact the approximation to determine the contact stiffness is a similar analysis to the contact between cylindrical roller bearings. Johnson [20] from the University of Cambridge analysis different types of contact mechanics. The stiffness is defined to be the relationship between the contact force and the displacement between the two bodies in contact. The equations for a parameter called the load-stress contact factor is derived from Hertz's equations.

$$
\begin{aligned}
b & =\sqrt{\frac{2 F}{\pi l} \frac{\left(1-v_{1}^{2}\right) / E_{1}+\left(1-v_{2}^{2}\right) / E_{2}}{\left(1 / d_{1}\right)+\left(1 / d_{2}\right)}} \\
p_{\max } & =\frac{2 F}{\pi b l}
\end{aligned}
$$

These equations are common equations used to define parameters and scaling factors in different types of contact analyses [29].

From these equations and the estimated contact force a composite elastic modulus is determined as a relationship between the two materials in contact.

$$
E^{*}=\frac{2 * E_{1} * E_{2}}{\left(1-v_{1}^{2}\right) * E_{2}+\left(1-v_{2}^{2}\right) * E_{1}}
$$

The composite modulus does not account for dynamic properties which will be present in a gear contact mesh. This modulus will only create a rough estimation of the stiffness. The gear contact modulus must be approximated with constant due to the nature of impact parameters required by 
Adams. The contact stiffness used for this simulation is used for the gear interaction is $5.0^{*} 10^{6}$ $\mathrm{N}-\mathrm{mm}[11]$.

\subsection{SYSTEM TORQUE}

At each stage of the assembly, there is an input and an output torque. The output torque will be reciprocated into the stage as a resistive torque. The resistive torque is equivalent to the torque seen in the system. The overall full system torque can be determined through from specifications provided by the Nordex N90 data tables [1]. This torque representation is shown below in Figure 20 and Table 9.

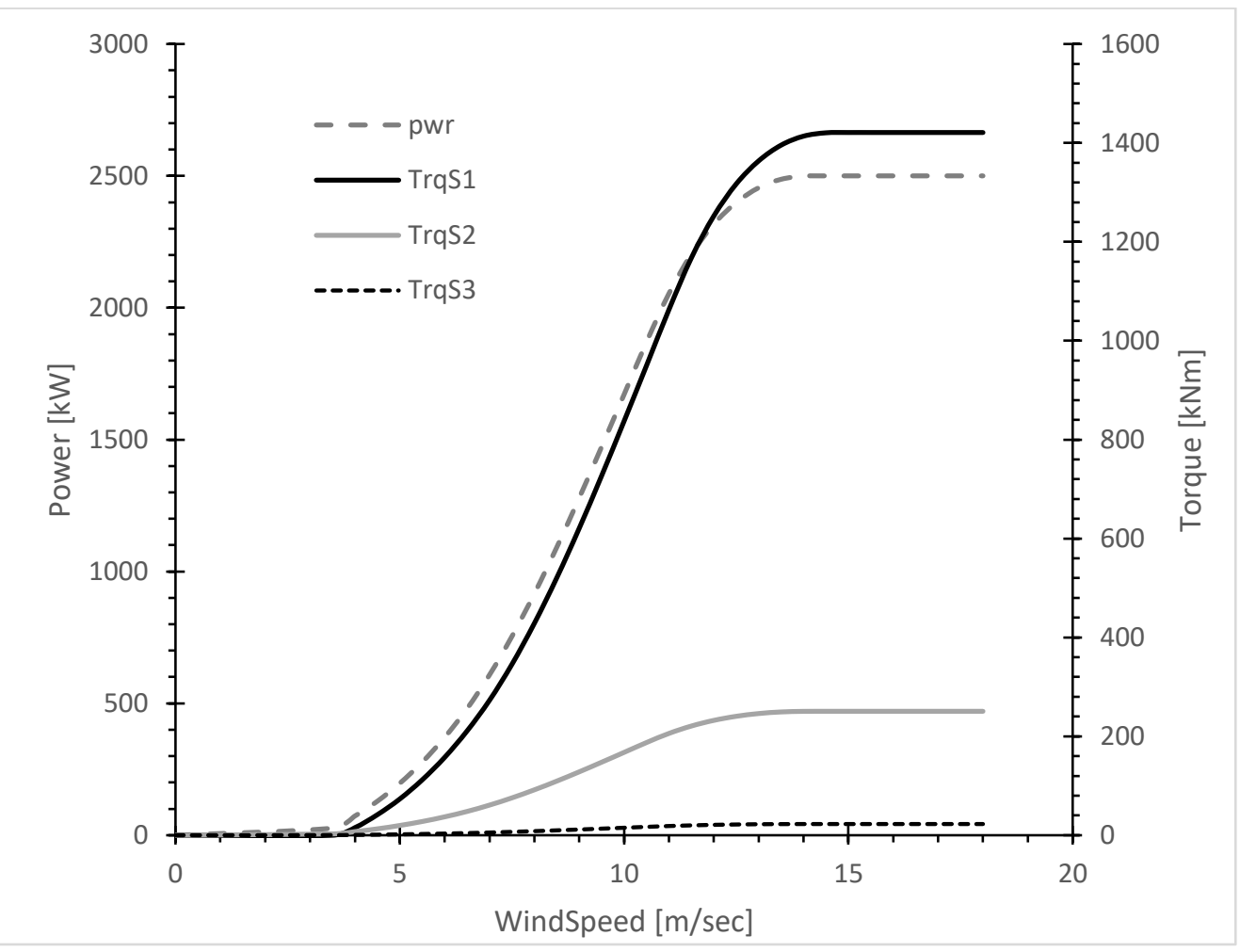

Figure 20. Torque and System Power as a function of wind speed 
Table 9. Torque and System Power

\begin{tabular}{|c|c|c|c|c|c|c|}
\hline $\begin{array}{c}\text { Wind Speed } \\
\mathrm{m} / \mathrm{s}\end{array}$ & $\begin{array}{c}\text { Power } \\
\mathrm{kW}\end{array}$ & $\begin{array}{l}\text { Speed } \\
\mathrm{rad} / \mathrm{sec}\end{array}$ & $\mathrm{S} 1 \mathrm{in}$ & $\mathrm{S} 2 \mathrm{in}$ & $\mathrm{kNm}$ & Out \\
\hline & 0.0 & 0.0 & 0.0 & 0.00 & 0.00 & 0.00 \\
\hline 0 & 0.0 & 0.0 & 0.0 & 0.00 & 0.00 & 0.00 \\
\hline 3.5 & 27 & 1.76 & 15.3 & 2.71 & 0.24 & 0.20 \\
\hline 4 & 73 & 1.76 & 41.5 & 7.32 & 0.66 & 0.53 \\
\hline 4.5 & 129 & 1.76 & 73.3 & 12.93 & 1.17 & 0.94 \\
\hline 5 & 197 & 1.76 & 112.0 & 19.75 & 1.79 & 1.43 \\
\hline 5.5 & 277 & 1.76 & 157.4 & 27.77 & 2.51 & 2.01 \\
\hline 6 & 371 & 1.76 & 210.9 & 37.19 & 3.36 & 2.69 \\
\hline 6.5 & 480 & 1.76 & 272.8 & 48.12 & 4.35 & 3.48 \\
\hline 7 & 608 & 1.76 & 345.6 & 60.95 & 5.51 & 4.41 \\
\hline 7.5 & 754 & 1.76 & 428.6 & 75.59 & 6.83 & 5.47 \\
\hline 8 & 916 & 1.76 & 520.7 & 91.83 & 8.30 & 6.64 \\
\hline 8.5 & 1092 & 1.76 & 620.7 & 109.47 & 9.90 & 7.92 \\
\hline 9 & 1279 & 1.76 & 727.0 & 128.22 & 11.59 & 9.27 \\
\hline 9.5 & 1473 & 1.76 & 837.3 & 147.67 & 13.35 & 10.68 \\
\hline 10 & 1671 & 1.76 & 949.8 & 167.52 & 15.15 & 12.12 \\
\hline 10.5 & 1870 & 1.76 & 1062.9 & 187.47 & 16.95 & 13.56 \\
\hline 11 & 2054 & 1.76 & 1167.5 & 205.91 & 18.62 & 14.89 \\
\hline 11.5 & 2203 & 1.76 & 1252.2 & 220.85 & 19.97 & 15.97 \\
\hline 12 & 2317 & 1.76 & 1317.0 & 232.28 & 21.00 & 16.80 \\
\hline 12.5 & 2399 & 1.76 & 1363.6 & 240.50 & 21.74 & 17.40 \\
\hline 13 & 2455 & 1.76 & 1395.4 & 246.11 & 22.25 & 17.80 \\
\hline 13.5 & 2487 & 1.76 & 1413.6 & 249.32 & 22.54 & 18.03 \\
\hline 14 & 2499 & 1.76 & 1420.5 & 250.52 & 22.65 & 18.12 \\
\hline 14.5 & 2500 & 1.76 & 1421.0 & 250.62 & 22.66 & 18.13 \\
\hline 15 & 2500 & 1.76 & 1421.0 & 250.62 & 22.66 & 18.13 \\
\hline 15.5 & 2500 & 1.76 & 1421.0 & 250.62 & 22.66 & 18.13 \\
\hline 16 & 2500 & 1.76 & 1421.0 & 250.62 & 22.66 & 18.13 \\
\hline 16.5 & 2500 & 1.76 & 1421.0 & 250.62 & 22.66 & 18.13 \\
\hline 17 & 2500 & 1.76 & 1421.0 & 250.62 & 22.66 & 18.13 \\
\hline 17.5 & 2500 & 1.76 & 1421.0 & 250.62 & 22.66 & 18.13 \\
\hline 18 & 2500 & 1.76 & 1421.0 & 250.62 & 22.66 & 18.13 \\
\hline
\end{tabular}

The modeling methodology for input into the system includes two different approaches: a torque input parameter or static angular velocity input. A static angular velocity input involves the application of a motor element on a joint in Adams with accompanying resistive torque applied 
to generate the contact force at each member of the assembly. Static motion input holds constant angular velocity at the input. This angular velocity input is method in which this analysis has been developed on. Although in certain cases, an input torque can be regarded as more realistic as an input to the gear train, the input motion at steady state on the gear stages still remains accurate to a wind turbine input parameter since the torque is represented through the system stages.

\subsection{SCALED MODEL}

The scaled model is created for simplicity of simulation and computational time. The model parts have a reduced gear module and thickness as well as a simplification of the connections in the system. The modifications made to the gear assembly can be seen in Figure 21 .

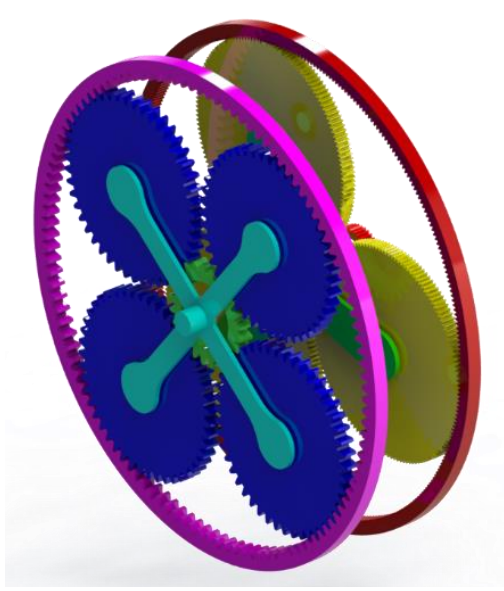

Figure 21. Full Assembly Half Scale Model - Solidworks Render

This model was imported to Adams in a similar method and definite of material. The Adams model is shown in Figure 22. Some material was later removed to increase computational efficiency. 


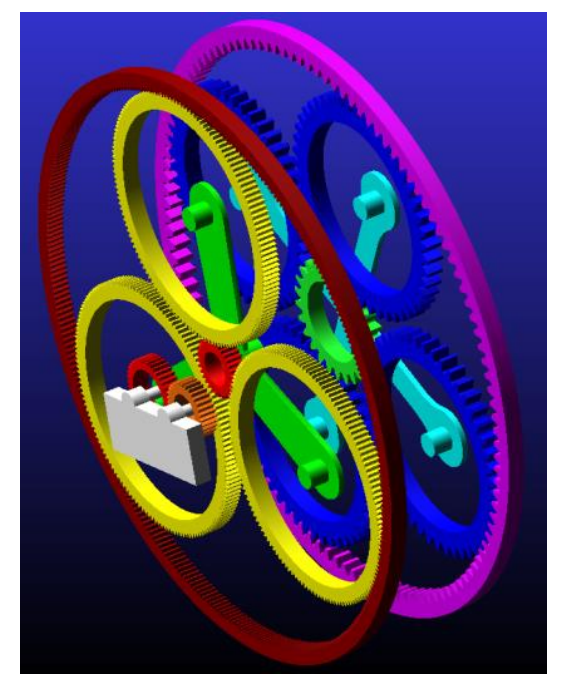

Figure 22. Full Assembly Half Scaled Model - Adams

The simplified assembly is constructed with the same types of joints and constraints, however there must be additional constraints added to relate each stage to the previous. The full joint set up is show in Table 10. 
Table 10. Half Scale Solid Body Joints

\begin{tabular}{|c|c|c|c|c|}
\hline Stage & Type & Body 1 & Body 2 & $\begin{array}{c}\text { Location } \\
\text { (Centered) }\end{array}$ \\
\hline \multirow{3}{*}{3} & \multirow[b]{2}{*}{ Lock } & Support & Ground & Ground \\
\hline & & Input (gear) & Sun Stage 2 & $\begin{array}{c}\text { Sun Stage } 2 \\
\text { center }\end{array}$ \\
\hline & Revolute & Output (pinion) & Support & Ground \\
\hline \multirow{5}{*}{2} & \multirow[b]{2}{*}{ Lock } & Ring 2 & Ground & Ground \\
\hline & & Carrier 2 & Sun Stage 1 & $\begin{array}{c}\text { Sun Stage } 1 \\
\text { center }\end{array}$ \\
\hline & \multirow{3}{*}{ Revolute } & Planet 2.1 & Carrier 2 & $\begin{array}{c}\text { Carrier } 2 \text { Pin } \\
\text { axis }\end{array}$ \\
\hline & & Planet 2.2 & Carrier 2 & $\begin{array}{c}\text { Carrier } 2 \text { Pin } \\
\text { axis }\end{array}$ \\
\hline & & Planet 2.3 & Carrier 2 & $\begin{array}{c}\text { Carrier } 2 \text { Pin } \\
\text { axis }\end{array}$ \\
\hline \multirow{6}{*}{1} & Lock & Ring 1 & Ground & Ground \\
\hline & \multirow{5}{*}{ Revolute } & Carrier 1 & Sun 1 & Ring 1 center \\
\hline & & Planet 1.1 & Carrier 1 & $\begin{array}{c}\text { Carrier 1 Pin } \\
\text { axis }\end{array}$ \\
\hline & & Planet 1.2 & Carrier 1 & $\begin{array}{c}\text { Carrier 1 Pin } \\
\text { axis }\end{array}$ \\
\hline & & Planet 1.3 & Carrier 1 & $\begin{array}{c}\text { Carrier 1 Pin } \\
\text { axis }\end{array}$ \\
\hline & & Planet 1.4 & Carrier 1 & $\begin{array}{l}\text { Carrier 1 Pin } \\
\text { axis }\end{array}$ \\
\hline
\end{tabular}




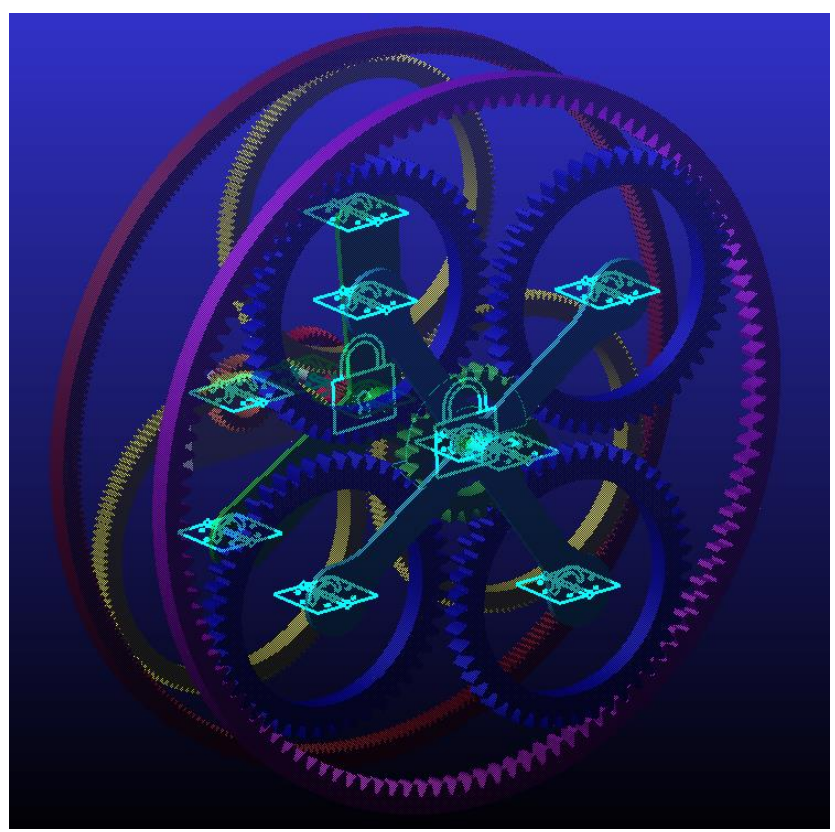

Figure 23. Adams model with joint locations

The elimination of the shafts on the model parts creates two different parts for the first stage sun gear and the second stage carrier as well as the second stage sun gear and the third stage input gear. In Table 10, it can be seen that a rigid joint is created in the method of a fixed joint to constraint the revolution of the first stage sun gear to the second stage carrier and the second stage carrier to the third stage input gear. The fix joint creates a ridged body constraint in between the two bodies, this can be similarly represented with a rigid shaft.

\subsection{SCALED MODEL MOTIVATION}

The scaled model requires a modification of any resistive torque or torque input into the system. The motivation for the scaled model is to reduce the effective computational time of the model analysis but represent similar stress patterns in the process. The scaling method used to estimate similar stress behavior is a static analysis of a cantilever beam similar to that performed to estimate the range of the stress to be expected in the gear root. The governing equation for beam bending stress is: 


$$
\sigma=\frac{M * y}{I}
$$

The bending moment $\mathrm{M}$ in $\mathrm{EQ}(\#)$, is representative of the tangential component of the contact force between the meshing teeth. For a gear at half the module the moment arm is reduced by half the original since the pitch radius is reduced by half. When analyzing for maximum stress $y$ is half the root thickness of the new gear tooth which is half the original thickness. The area moment of inertia for a beam is represented by:

$$
I=\frac{1}{12} * b * h^{3}
$$

The height of the beam is the root thickness of the tooth which is halved when the module is halved. Finally, $b$ represents the face width (gear body thickness). A relationship between the original model and a new beam model can be made while keeping the bending stress constant:

$$
\frac{F_{t 1} * r_{1} * y_{1}}{\frac{1}{12} * b_{1} h_{1}^{3}}=\frac{F_{t 2} * r_{2} * y_{2}}{\frac{1}{12} * b_{2} h_{2}^{3}}
$$

Substituting in for the relationship between the two models yields:

$$
\begin{gathered}
\frac{F_{t 1} * r_{1} * y_{1}}{\frac{1}{12} * b_{1} h_{1}^{3}}=\frac{F_{t 2} * \frac{r_{1}}{2} * \frac{y_{1}}{2}}{\frac{1}{12} * b_{2} *\left(\frac{h_{1}}{2}\right)^{3}} \\
F_{t 1}=\frac{2 F_{t 2} * b_{1}}{b_{2}}
\end{gathered}
$$

Converting the contact force into torque:

$$
\begin{aligned}
& F_{t 1} r_{p 1}=T_{1} \text { and } F_{t 2} r_{p 2}=T_{2} \\
& \frac{2 F_{t 2} * b_{1}}{b_{2}} * r_{p 1}=T_{1} \\
& \frac{\frac{2 T_{2}}{r_{p 2}} * b_{1}}{b_{2}} * r_{p 1}=T_{1}
\end{aligned}
$$




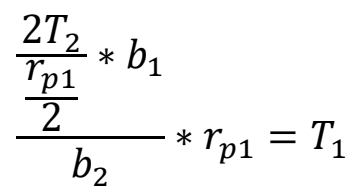

$$
\begin{aligned}
& 4 T_{2}\left(\frac{b_{1}}{b_{2}}\right)=T_{1}
\end{aligned}
$$

EQ (12)

The equation above shows an approximated torque scaling factor between a single fix axis gear mesh with and an undefined face width scale factor.

The scaled modal also eliminates a portion of material at the center of the gear. An analysis was performed on a slice of the gear body to determine the stress at the center of the gear as it undergoes a static analysis from an applied simulated contact force. This analysis is show in Figure 24.

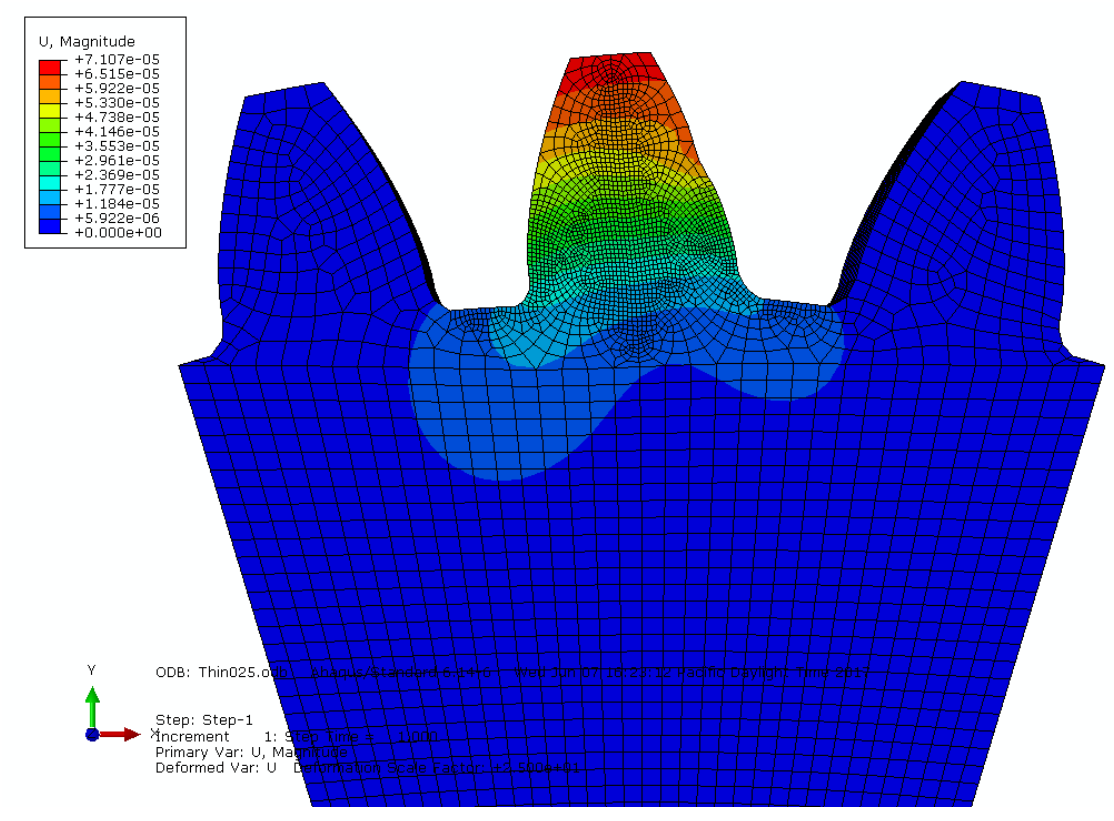

Figure 24. Gear stress contour plot

Figure 24 shows the stress profile of a gear tooth from a portion of the gear due an applied force at the contact patch. It can be seen that there is negligible or no deformation towards the center 
of the gear body. This allows for the modeling of the gear body while neglecting the center portion of the gear material. 


\section{CHAPTER 5. DEFECT MODEL}

\subsection{CRACK/NOTCH MODEL DEFECT}

Different models are created in preparation for different stage analysis of the system. The sun gear one the first stage is created with two different configurations; one representing a fully

healthy gear and the other with a defect at the root of the gear tooth. As a preliminary analysis of this type of defect, the defect is created to extend approximately a quarter of the root thickness of the gear. These two different configurations are shown in Figures 25 and 26 


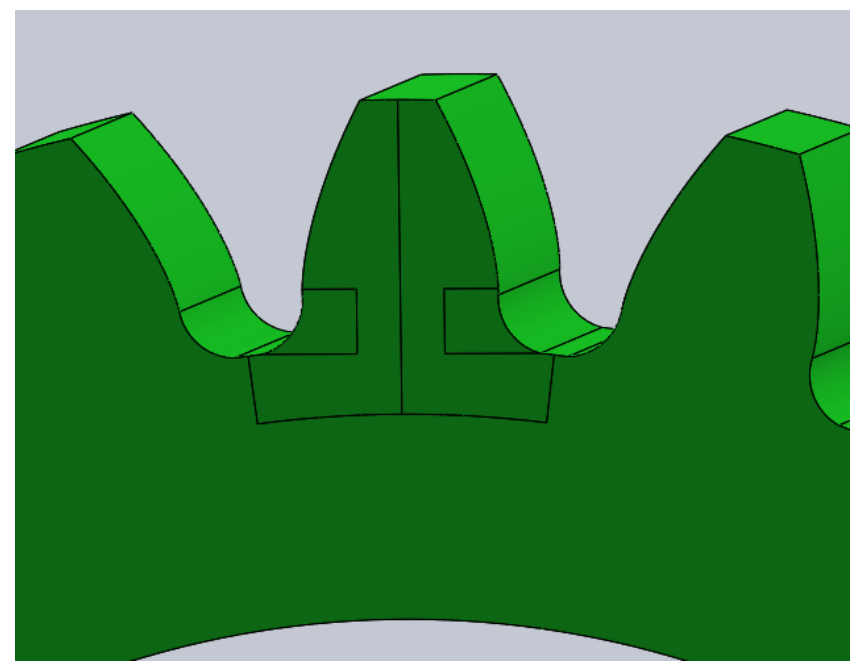

Figure 25. Configuration 1 - no defect

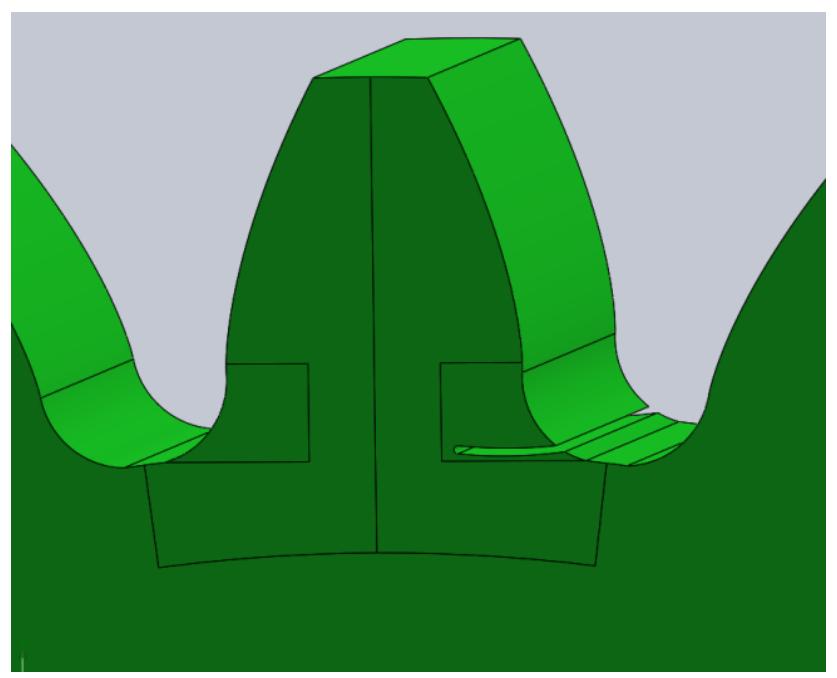

Figure 26. Configuration 2 - defect

The defect created remains within the split face partition created in Solidworks for a mesh refinement location. For increasing notch length the partition can be varied in order to create the necessary fine mesh at the internal radius of the defect in the gear tooth. This model is imported into Patran to develop a finite element model and generate a modal neutral file for Adams. The mesh development follows the same ideology as described in the previous section on Finite Element Model. The mesh on the defect tooth is show in Figure 27. 


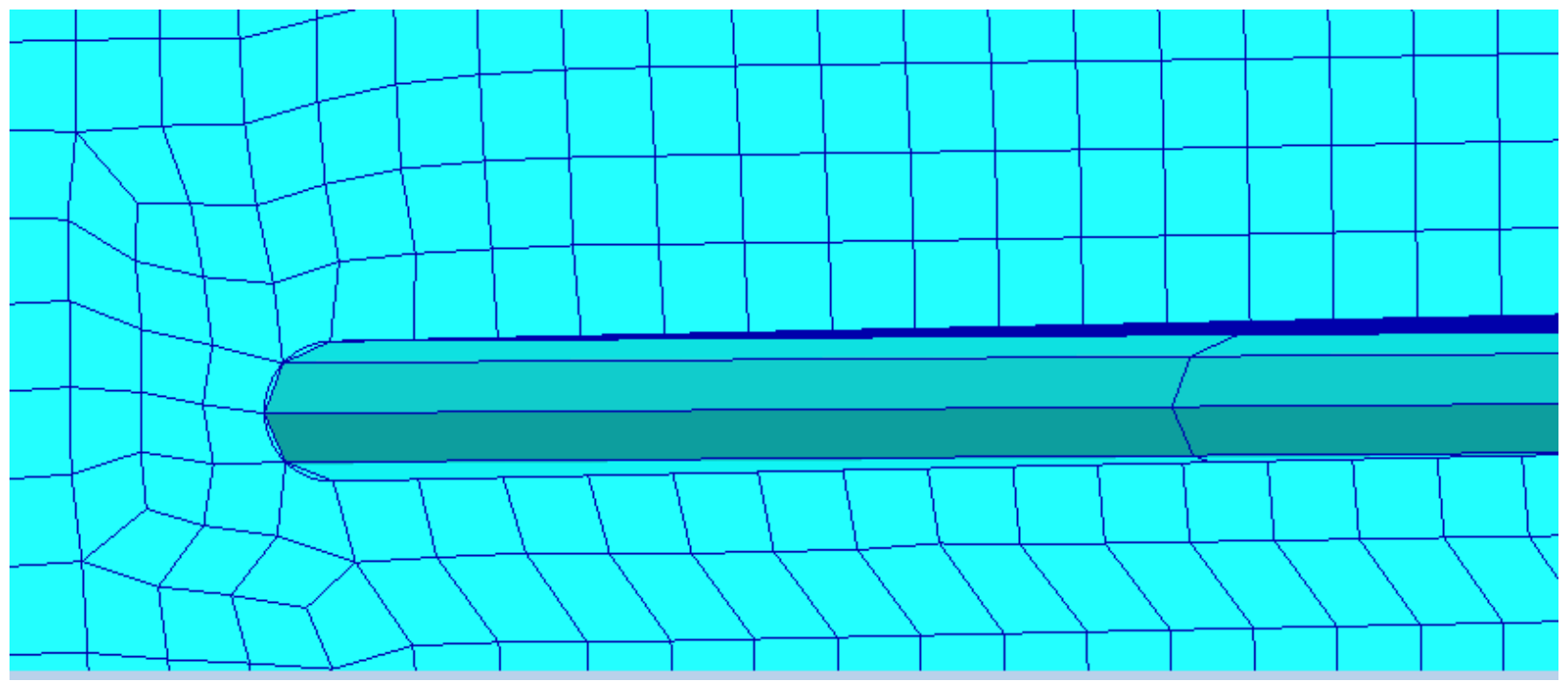

Figure 27. Notch radius mesh refinement at the sun gear of the first stage

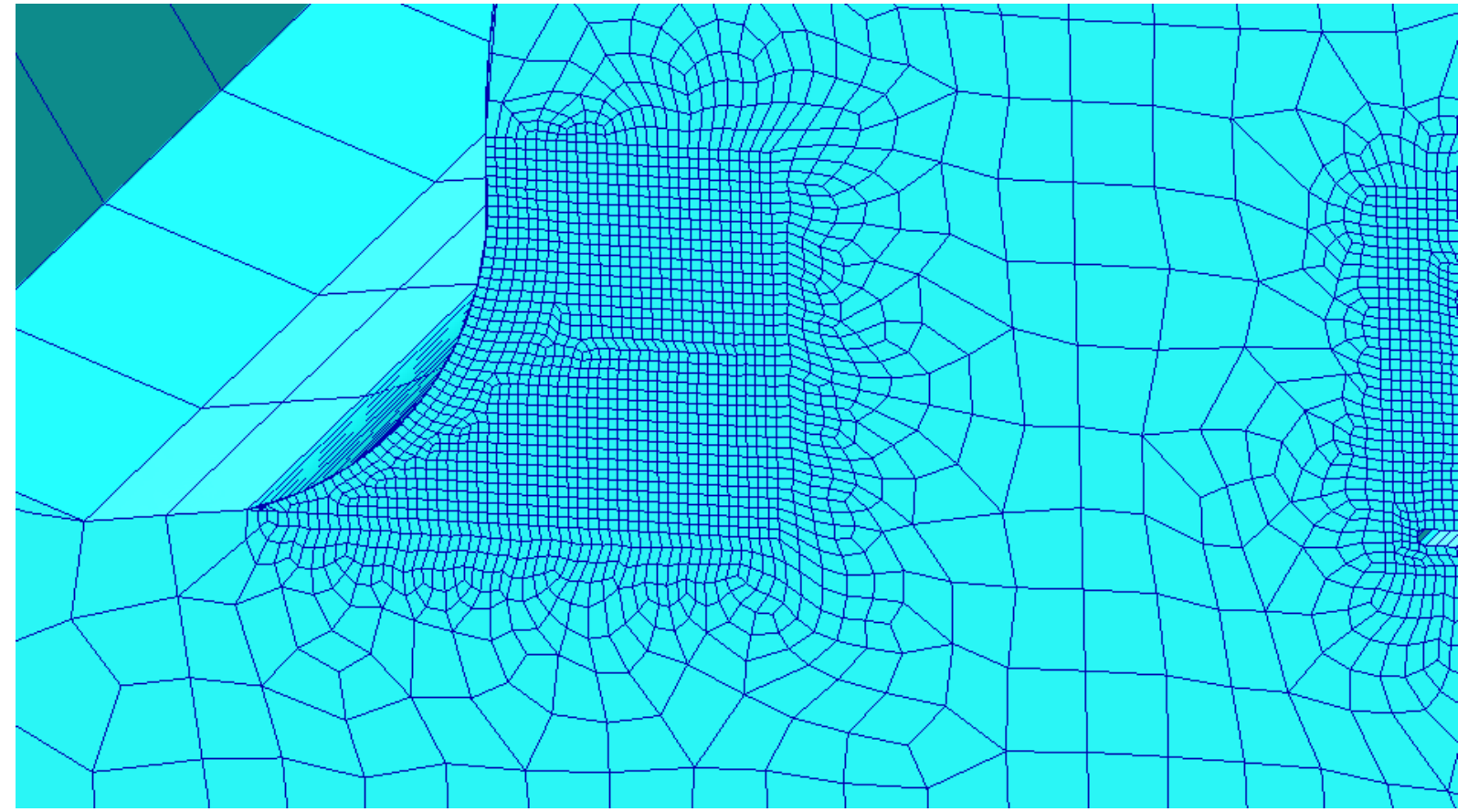

Figure 28. Opposite side root mesh for compressive stress analysis

Although, it is important to determine that the mesh has reached convergence to produce

accurate stress results, it must be noted at this point that a defect in the gear tooth is indicative of gear tooth failure which in turn would results yielding and fracture of the material at the gear root. This results in an increase stress profile at the notch radius location as the model does now 
allow for dynamic crack propagation and produce stresses which will exceed the allowable stress in the material. The resulting overall effect at this location is non-linear behavior as, realistically, the geometry change will exceed that which is within the assumption of small angle and small displacement for linear finite element analysis to remain accurate. This behavior, in reality, will cause the tooth to plastically deform and deflect further with each force application of force. The propagation of the crack across the thickness of the root will result in the reduction of the effective area moment of inertia at the root of the gear, resulting in higher stress.

With this in mind, meaningful data can still be extracted from the results, most significant of all the vibration patterns. The theory of modal superposition which is the theory behind the integration of the modal analysis in Patran/Nastran into Adams is a based on a linear superposition of different mode shapes and assumes a linear theory. This prevents crack propagation using a method in Patran called 'unglue elements' and 'break nodes' and application of non-linear material properties to capture the true non-linearity of the behavior. The method of 'unglue elements' and 'break nodes' is a different method to create cracks. Instead of manually removing material in the CAD model, these two methods create overlapping nodes in the same location which can behave independently. Different defect models are created with a defect applied at the sun gear on the first stage and creating an assembly with multiple stages to analyze the output. In this case, the three models under analysis includes a single first stage model, a two stage planetary model, and finally a full system model with the first stage sun gear defect. 


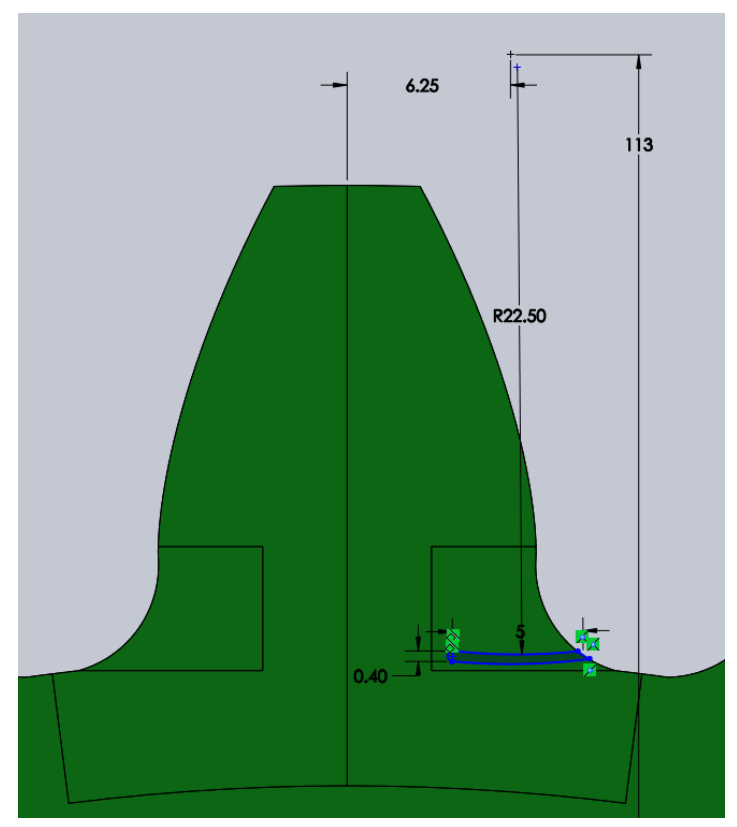

Figure 29. Defect parameters dimensions

Figure 29 shows the dimensions for the notch which is applied at the root of the gear tooth. This notch has length $25 \%$ of the root thickness of the tooth, in this case the root thickness is $20 \mathrm{~mm}$ and the notch length is $5 \mathrm{~mm}$. The notch has a slight curvature and is created with an offset inside of Solidworks. The thickness of the notch is made to be $10 \%$ of the length across the tooth face.

\subsection{ADAMS VIEW FLEX}

As mentioned in the Introduction, an Adams module is available for use which generates a flexible body without having to import a modal neutral file. Adams View Flex uses a basic internal meshing software which generates its own .mnf file through Nastran. View flex is used to general a flexible model to compare the results to Patran/Nastran. A full tutorial on the development of a flexible body through View Flex can been seen in Appendix 2.

View flex acts similarly as Patran does and can be treated as simply a different, simpler meshing software.

There are a number of limitations to the View Flex module in Adams. The overall basic meshing interface allows for the global element definition of the mesh to be defined. For gear model such 
as the one under analysis, there are two methods of meshing the part to obtain accurate stress representation; a biased mesh (the method that has been outline in Patran) or a fine mesh on the entire gear body. If high performance hardware were available to perform an analysis on a full body fine mesh on a gear tooth and body, this would not be a major concern. However, as an underlying goal in this analysis, developing a simplified model is meant to reduce computational time and increase analysis efficiency. Not only would the modal neutral file creation from View Flex take a significant amount of time, the dynamic simulation for a full stage assembly would also require a significant amount of time. The idea of meshing an entire gear body to such a refined mesh so as to match that required at the root of a tooth or the notch radius, would be highly inefficient. The meshing module for View Flex is shown in Figure 30. 


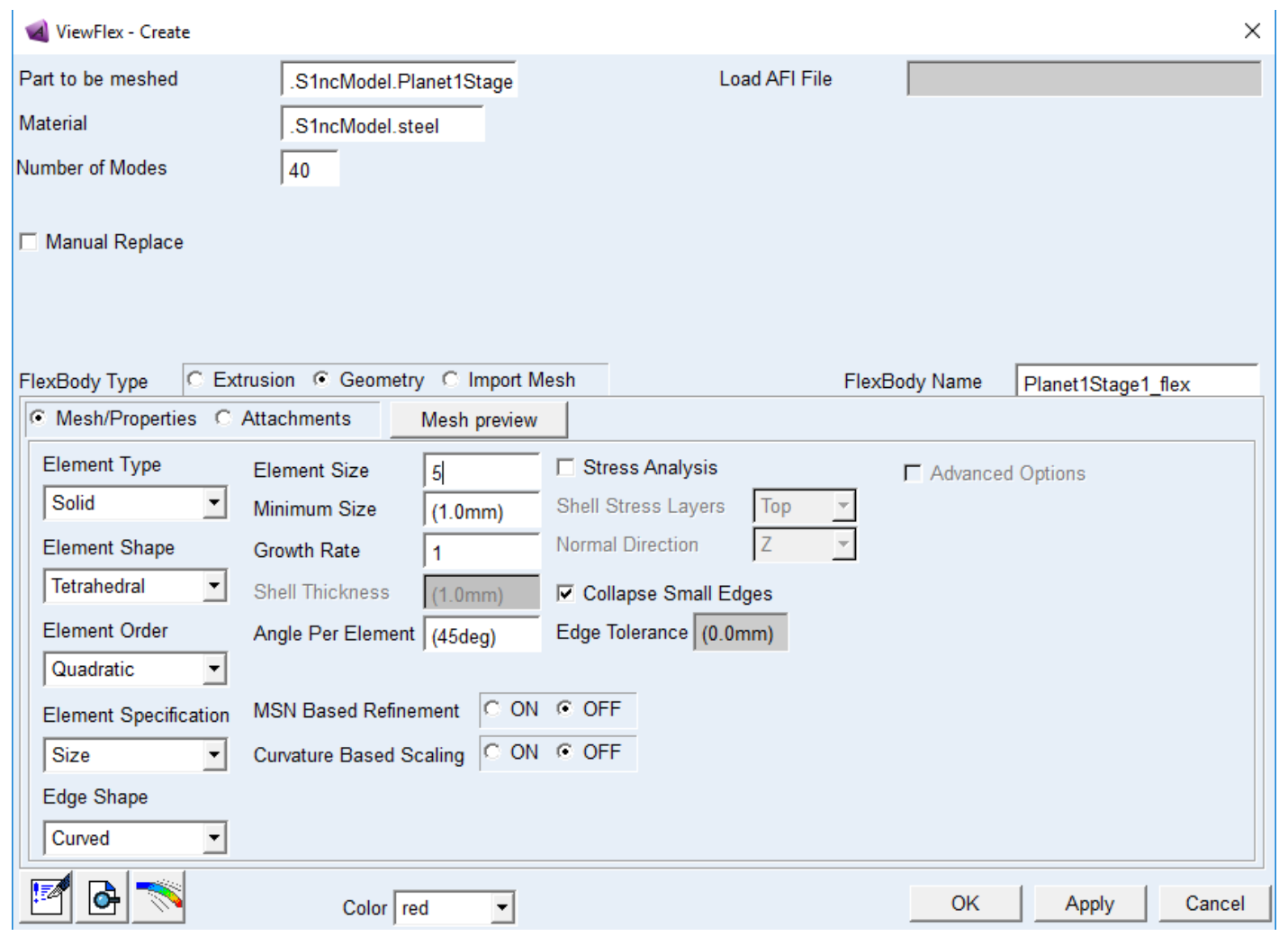

Figure 30. Adams Flex Control Module View

The meshing module includes the basic options to create a finite element model. As can be seen, the meshing module does not allow for the level of mesh refinement which can be achieve by a full FEA program. The element size selected was $5 \mathrm{~mm}$, a minimum size of $1 \mathrm{~mm}$ with a growth rate of $1 \mathrm{~mm}$. It is clear here that the minimum edge size is insufficient to capture the stress as previously analyzed by Abaqus convergence study.

For a more simplified geometry, View Flex can be ideal for obtaining a rough benchmark for analysis. This is similar to the meshing module for Solidworks. However, when a specific mesh is required with different partitions or seed biases a full FEA software is the better choice. With the right hardware resources however, View Flex may be able to generate a full body mesh to analyze. A full body mesh would allow for an accurate representation of the stress profile but it is an inefficient use of time and resources. Although the analysis of a simulation with View Flex 
will not be used to create a meaning full comparison with final results, a tutorial on View Flex can be found in Appendix 2. 


\section{CHAPTER 6. RESULTS}

\subsection{GEAR RATIO VERIFICATION}

The models generated in Adams allow for a multibody dynamic analysis of the system. In this case the gear system can be analyzed using a variety of parameters including a flexible body for stress analysis. The goal of the gear system analysis is to discover the vibration patterns from different stages of the planetary gearbox. The vibration pattern analyzed through a Fast Fourier Transform (FFT) plot will show different amplitudes and of certain vibrational frequencies resulting from a defect in a certain area of the gearbox.

The first test performed in Adams was to simply simulate the results of the predicted angular velocities of each critical component to determine if they match those predicted by the design parameters. Table 11 shows the predicted rotational velocity of each component.

Table 11. Calculated Component Velocities

\begin{tabular}{lccc} 
& Stage 1 & Stage 2 & Stage 3 \\
\hline Sun & 10.134 & 112.10 & - \\
Planet & 2.695 & 12.370 & - \\
Carrier & 1.76 & 10.134 & - \\
Ring & 0 & 0 & - \\
Gear & - & - & 112.08 \\
Pinion & - & - & 140.10 \\
\hline
\end{tabular}




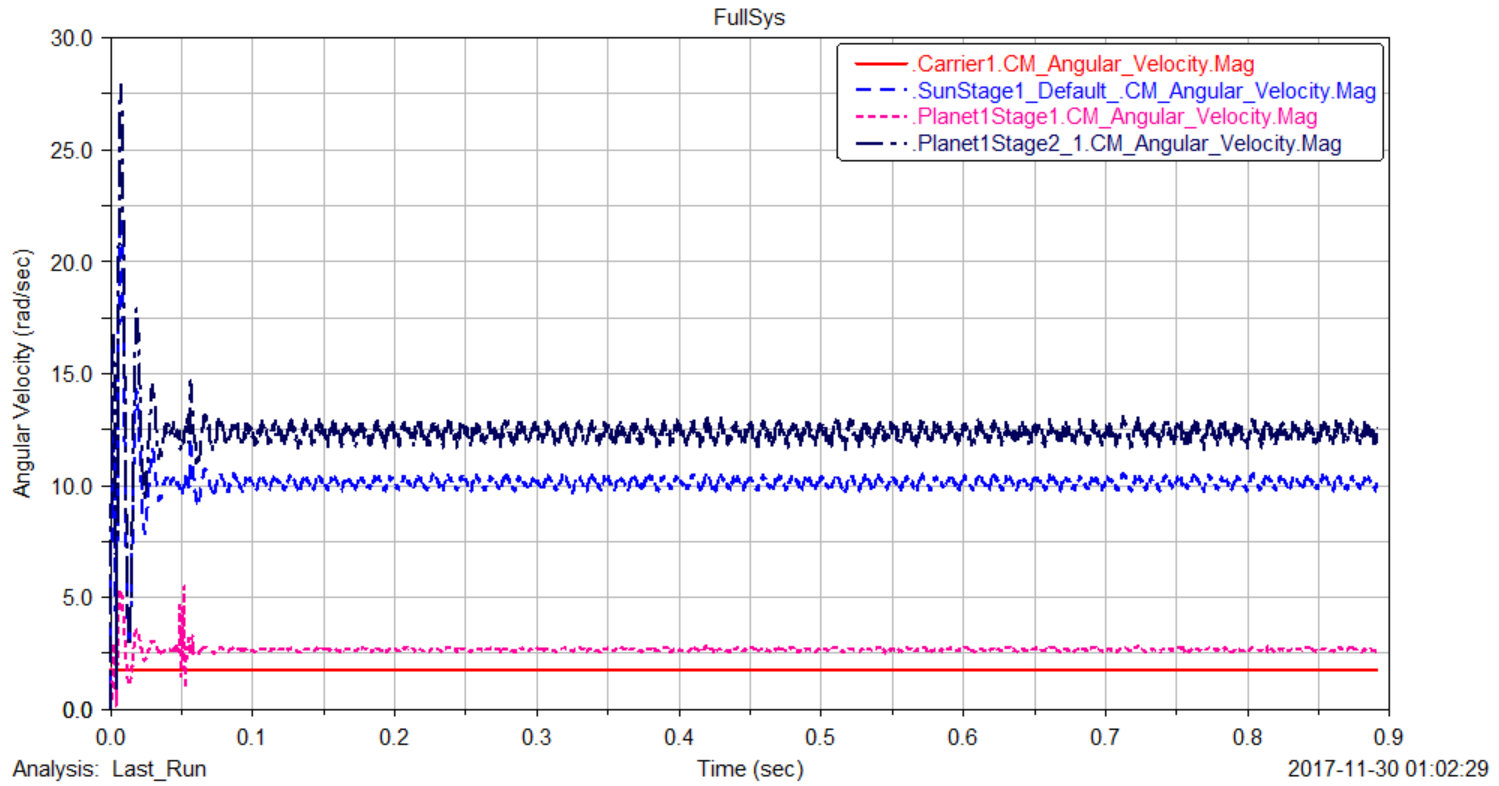

Figure 31. Stage 1 and Stage 2 Component Velocities

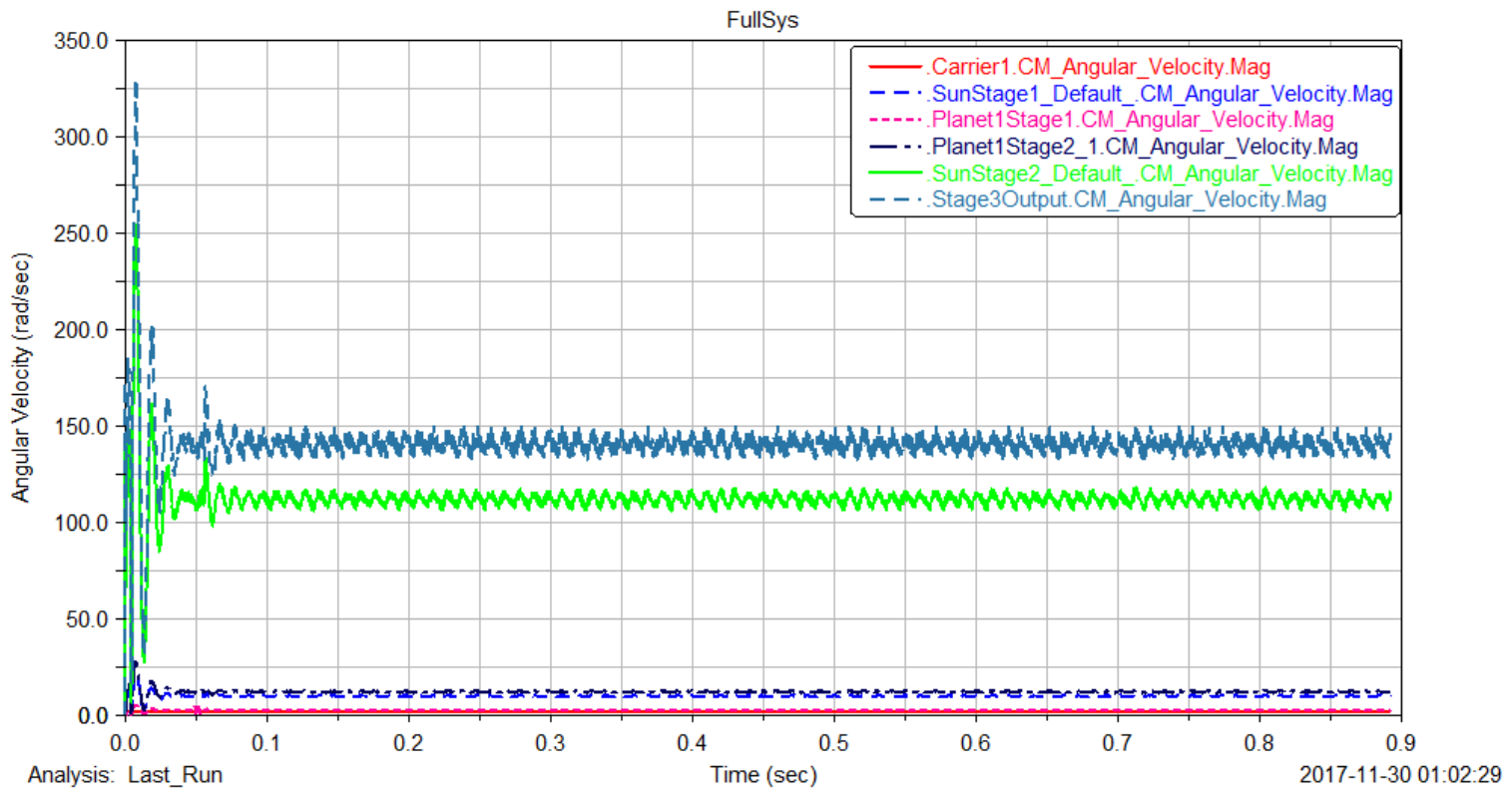

Figure 32. Three Stage velocities of all components

The two Figures 31 and 32 show that the angular velocity of each component matches those predicted by the model. These two plots establish a base line that the gearbox is functioning with the correct ratio between each stage to produce the required overall gear reduction. 


\subsection{STRESS ANALYSIS - STATIC}

As a preliminary stress results on the gear tooth were analyzed to capture a static behavior of a gear tooth under load with different defect sizes at the root. It must first be indicated that this analysis was performed in Abaqus incorporating non-linear material properties and behavior of the associated gear tooth. The non-linearity of the behavior of the material must be accounted for when analyzing failure from defects. The non-linear properties for the gear tooth material is presented in Figure 33.

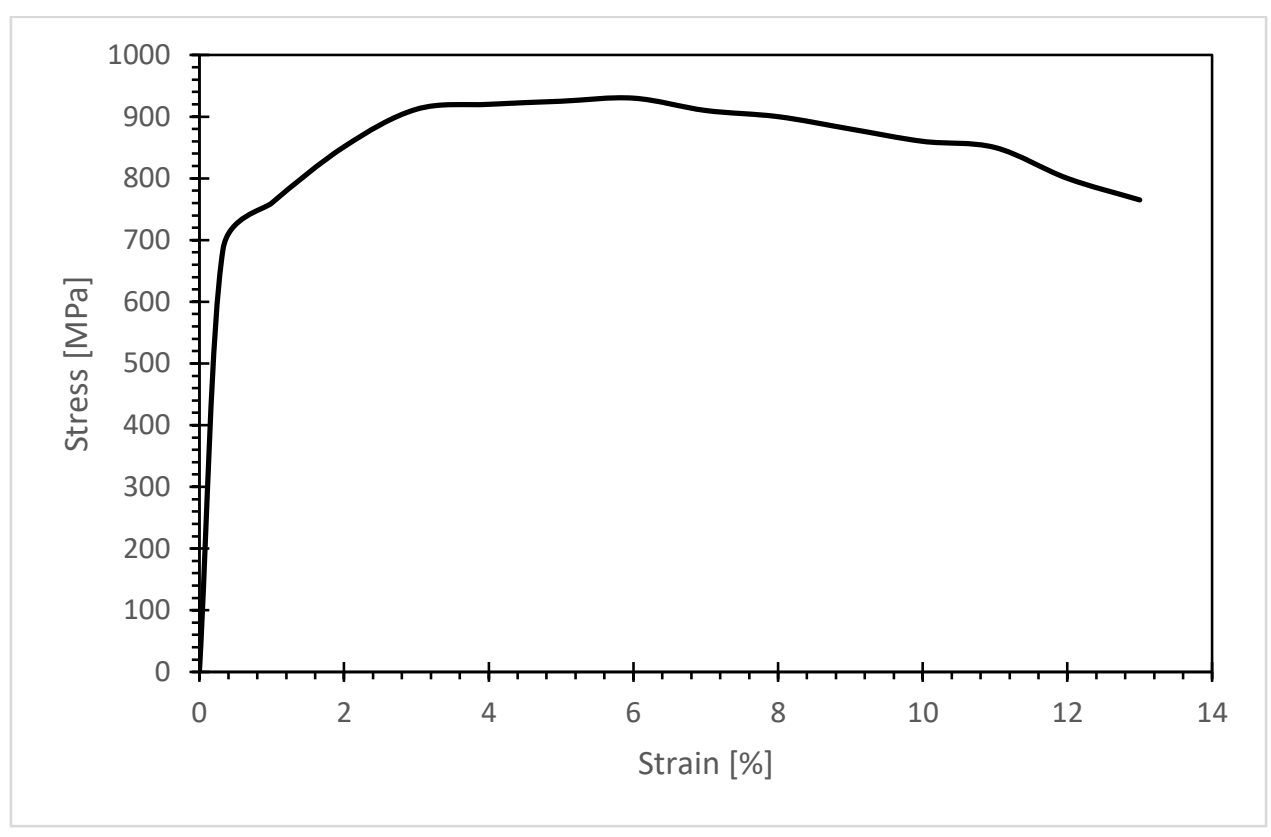

Figure 33. AISI 4820 Steel Estimated Non-Linear Stress vs. Strain Relationship The following plastic deformation properties were applied to the material in anticipation to capture material failure behavior. This plot was determined using the yield and ultimate stress as the critical locations with the combination of interpolation to other similar materials A set of static loading case was applied to the gear too with different notch lengths at the root. The following results are presented bellow in as Table 12 . 
Table 12. Static Analysis of a Sun Gear Tooth with varying defect size.

\begin{tabular}{ccccccc} 
Root Notch & Left & $\begin{array}{c}\text { Stress Location } \\
\text { Right }\end{array} \begin{array}{c}\text { Contact } \\
{[\%]}\end{array}$ & Notch & $\begin{array}{c}\text { Disp } \\
{[\mathrm{mm}]}\end{array}$ & Comments \\
\hline none & 361 & 312 & 366 & & 0.024 & $\begin{array}{c}\text { Elastic (operating } \\
\text { conditions) SF 1.25 } \\
\text { Yield at notch plastic } \\
\text { deformation. }\end{array}$ \\
25 & 455 & 140 & 302 & 726 & 0.035 & $\begin{array}{c}\text { Yield notch and left } \\
\text { fillet. }\end{array}$ \\
\hline \hline
\end{tabular}

In Table 12, the stress was measured using the Von Mises stress criteria at different locations on the gear tooth. These three locations include the left and right root of the gear tooth, the defect location, and the simulated "contact" location. The application of the contact load is applied as pressure over a contact patch. Figure 34 and 35 shows a depiction of one of the test cases.

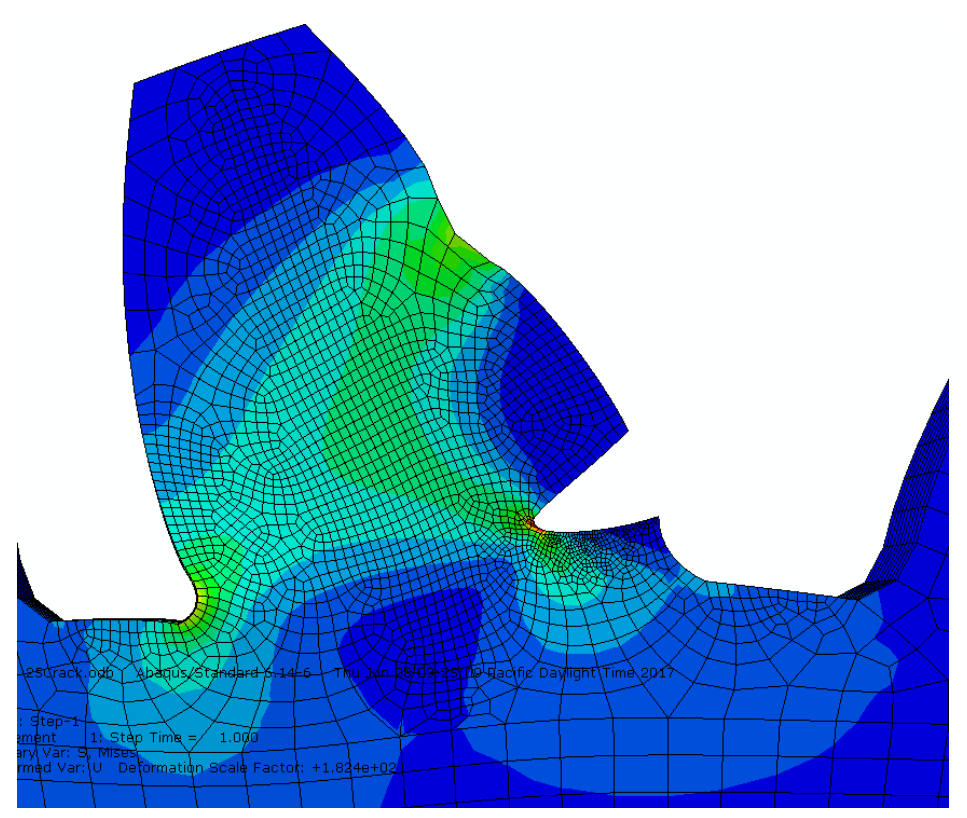

Figure 34. 25\% Root Notch Deformed Body Front View 


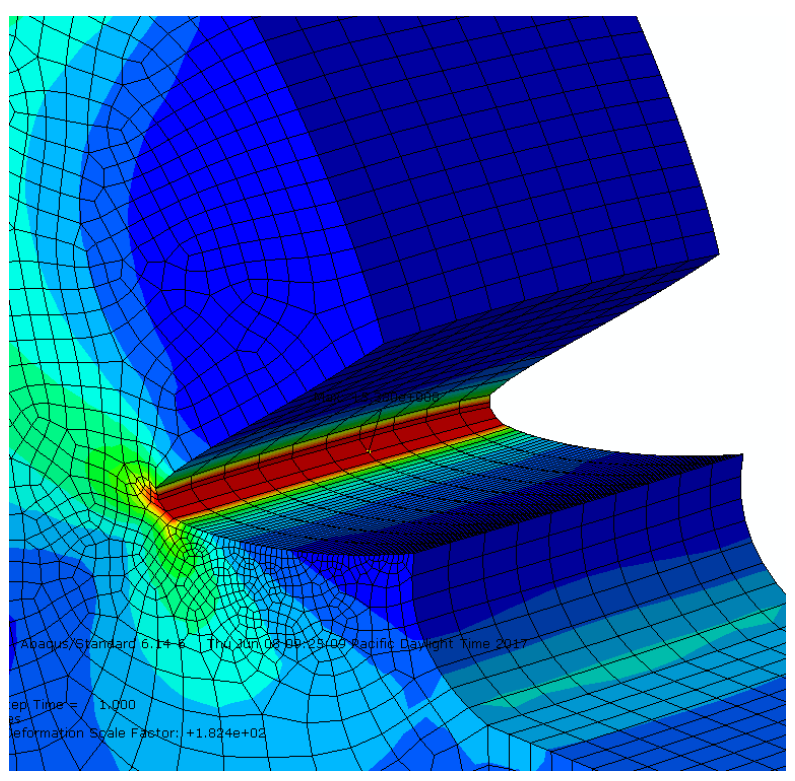

Figure 35. 25\% Root Notch Deformed Body Notch View

From Figures 34 and 35, the stress contour shows the three high stress location being the notch, the compression root location and the contact patch. It must be noted that the contact stress measurement must be read with caution. The FE program simulates a distributed pressure load as a series of point loads on a surface. The error associated with applied point loads is areas of infinite stress at the node of application. However, as the distance between the point of interest and the location of load application the solution converges rapidly [22]. Therefore, the true contact stress at the location may not be accurately capture by the FE model results.

From the first application of a defect at the root, the results show that material failure occurs at the notch location. TBC

\subsection{STRESS ANALYSIS - DYNAMIC}

The FE model creation was developed by Patran using the modal superposition model. The most significant factor in the stress analysis of the dynamic model is the non-representation of the non-linearity of the system. The static model is able to account for material non-linearity which 
is not available to modal superposition. Therefore, stresses measured in the dynamic model around or greater than the allowable yield stress must be taken with care.

The stress analysis at the root of the gear tooth can be performed under dynamic loading the gear tooth with the integration of the flexible body into Adams. The flexible body created was the sun gear on the first stage of the planetary system. The selection for one the stress location is shown in Figures 36 and 37.

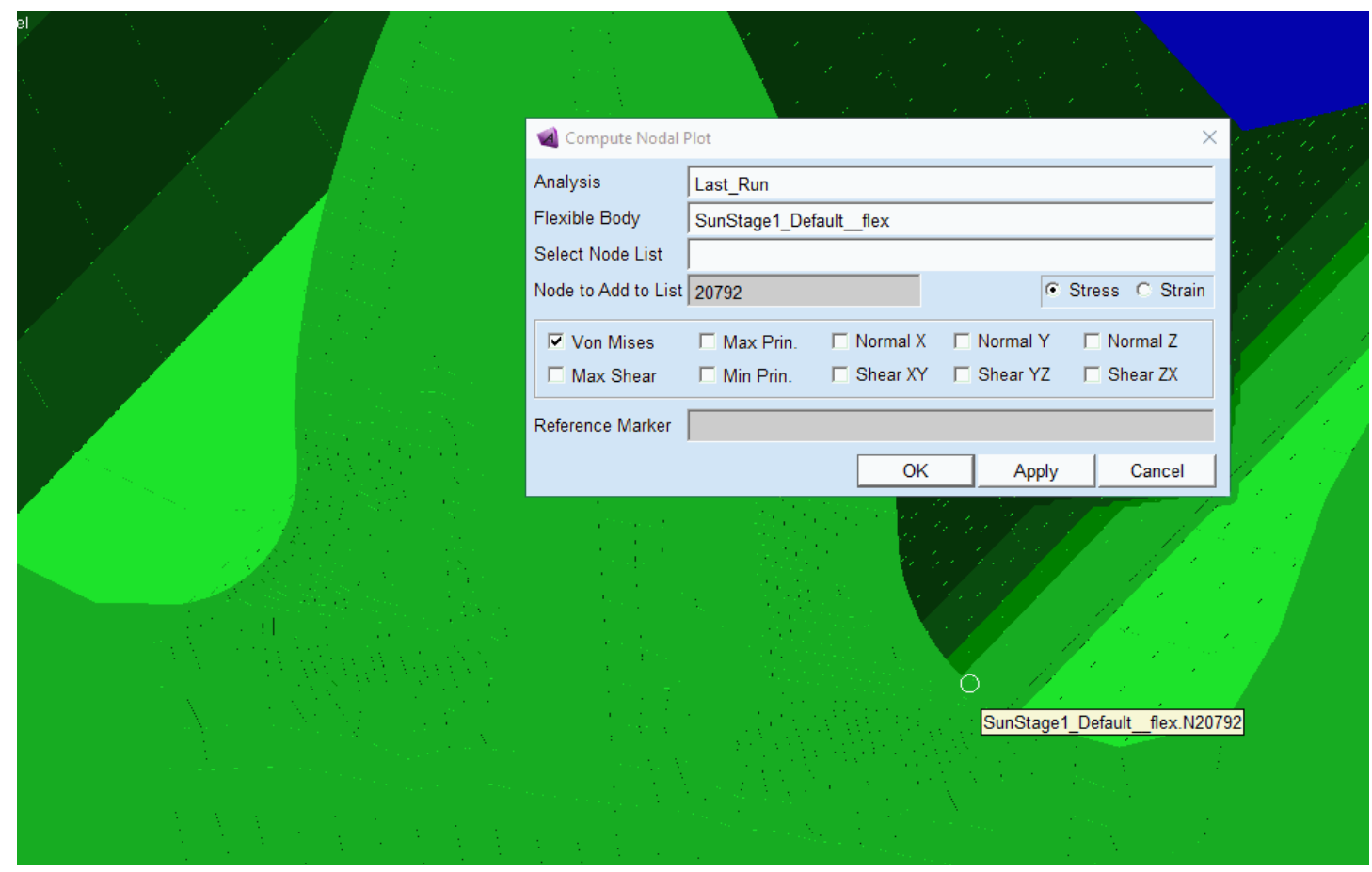

Figure 36. Stress node probing in Adams 


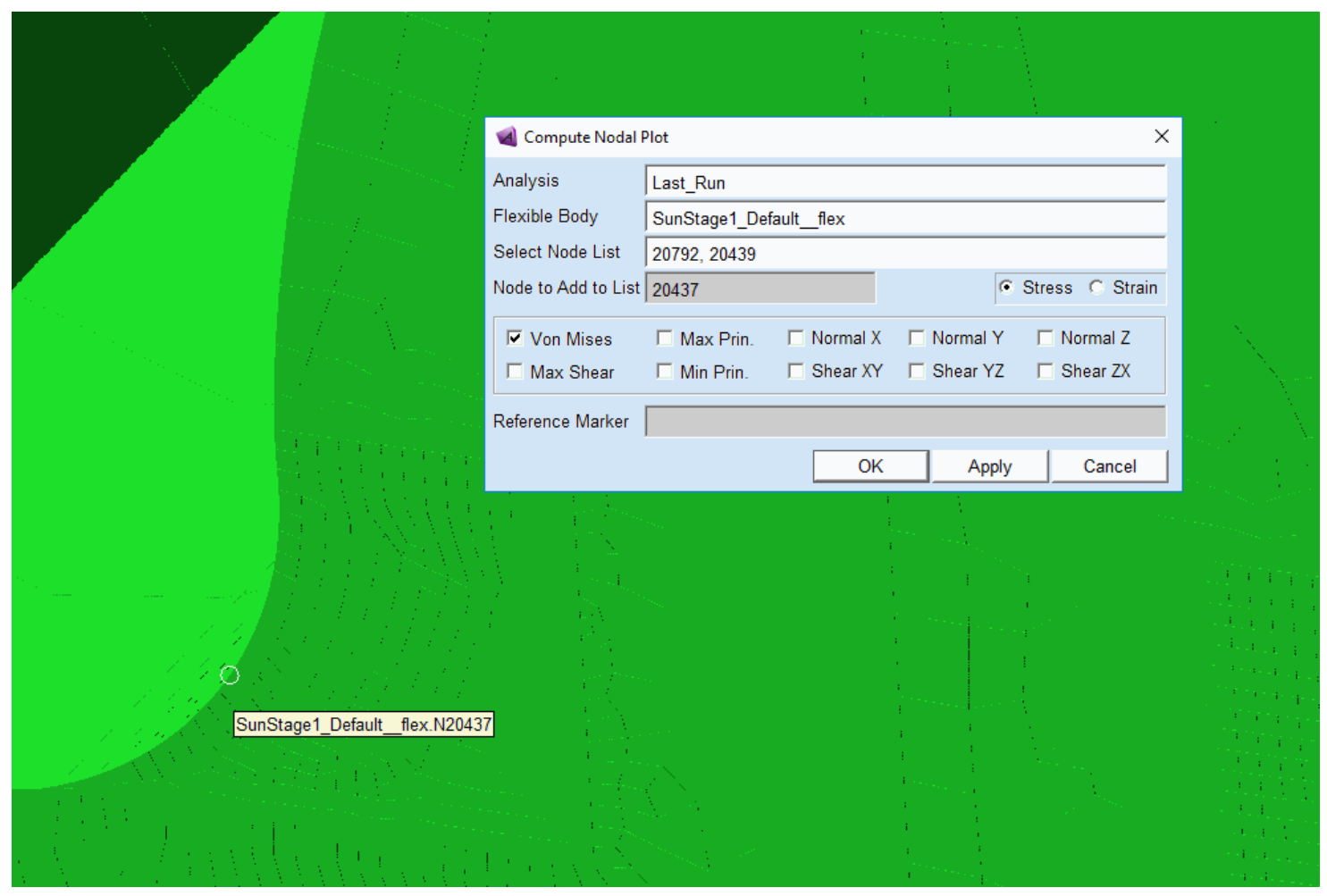

Figure 37. Stress node probe location 2

Using the Durability module in Adams, the stress locations can be probed in Adams and plotted as a function of time or another independent variable. The stress contour plot for a healthy tooth on the sun gear is shown in Figure 38. 
Max Prin. Stress (MPa)

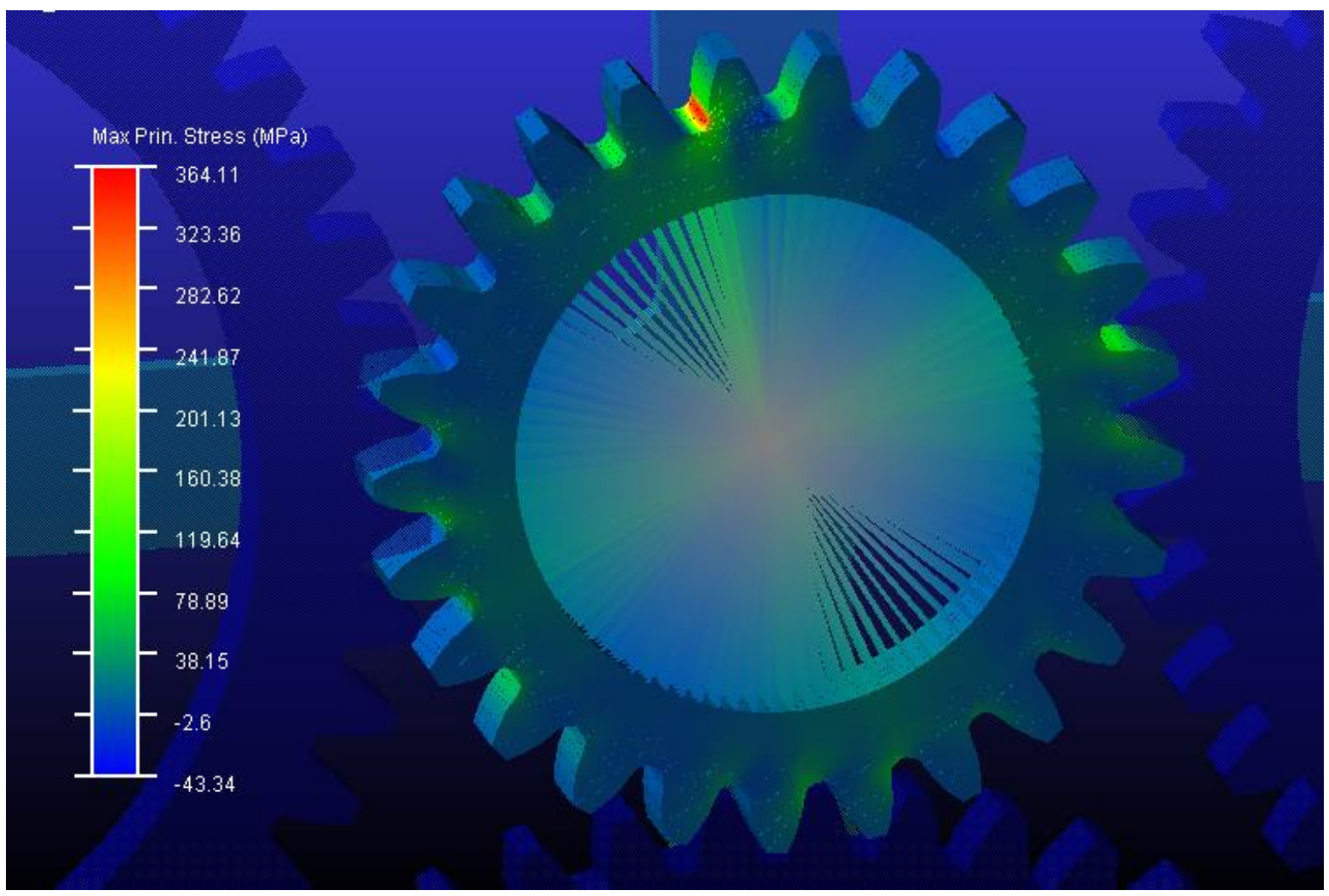

Figure 38. Stress contour plot in Adams

Figure 38 shows the plot for the maximum principle stress contour plot of the sun gear.

Displaying the maximum principle stress shows the tensile stress incurred on one of the roots of the gear. The tensile and compressive stress is expected to be different on the gear. The three stresses probed at these various locations are the maximum principle stress, minimum principle stress and the Von Mises stress criteria. A general deformation plot is shown in Figure 39. 


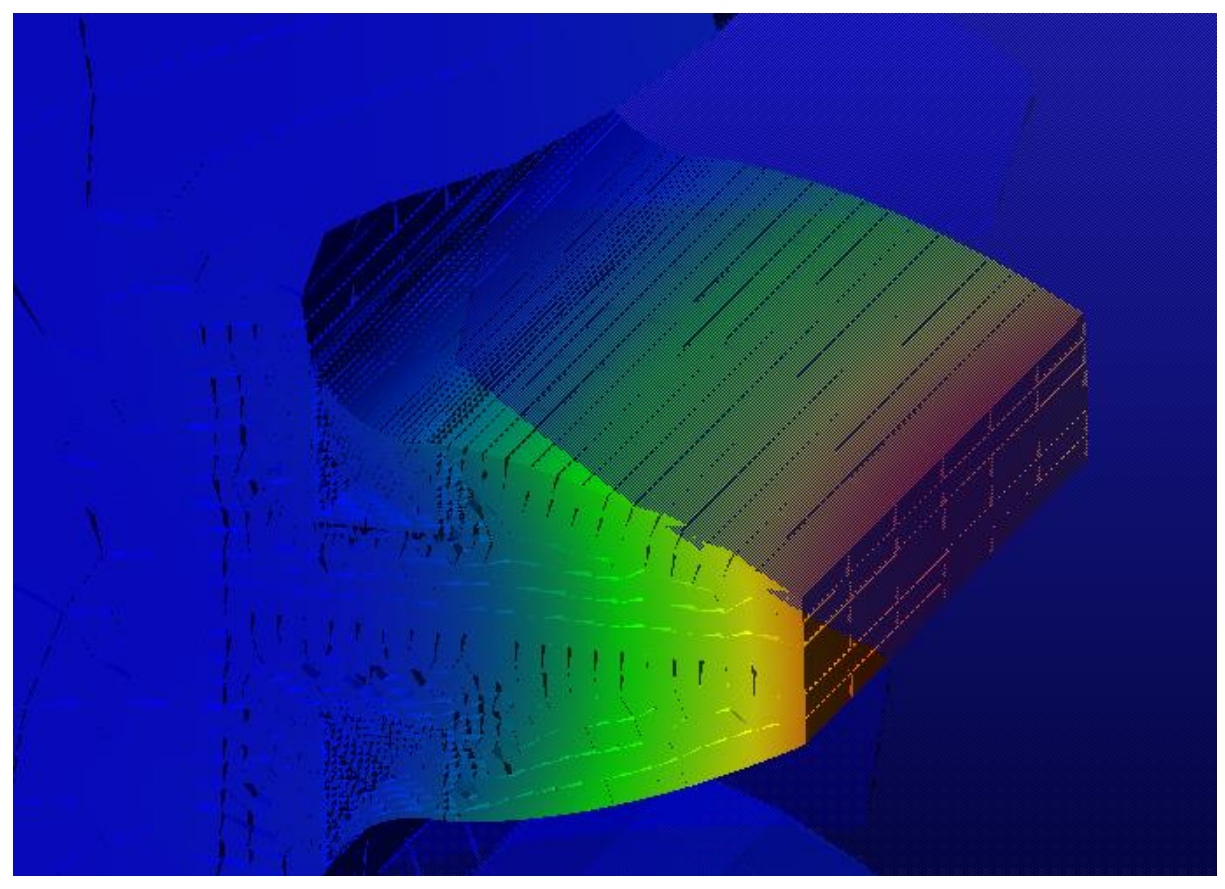

Figure 39. Stress contour plot of finely meshed tooth in Adams

Figure 39 stress plot shows the maximum principle stress at the root location for tensile stress.

The stress profile generated at the tooth shows the expected results. The area which exhibits the maximum stress point occurs at the root of the tooth. A series of nodes were probed to determine the stress at the root of the tooth. It can be seen that, as the stress not only varies across the height of the tooth, from base to tip, but also across the face width. This stress gradient is shown in Table 13. The general deformation gradient shown in Figure 39 shows the deflection at the tip of the tooth as it rotates through the mesh between each planet. The deformation gradient acts as predicted. The limitation to this plot is the lack of a contour gradient indicator. This is due to the nature of the interface methodology between the two programs Adams and Patran/Nastran and the content of the .mnf file link. 
Table 13. Stresses at six node locations of the gear tooth from the center to the face

\begin{tabular}{c|ccc}
$\begin{array}{c}\text { Node } \\
\text { Location }\end{array}$ & $\begin{array}{c}\text { Von } \\
\text { Mises }\end{array}$ & $\begin{array}{c}\text { Max Pri. } \\
{\left[\mathrm{N} / \mathrm{mm}^{\wedge} 2\right]}\end{array}$ & Min Pri. \\
\hline 10706 & 312.3 & -9.87 & -351.9 \\
18266 & 311.8 & -9.26 & -340.9 \\
20792 & 239.8 & -112.3 & -258.2 \\
10355 & 322.8 & 361.6 & 8.27 \\
12869 & 298.0 & 333.2 & 8.45 \\
20437 & 274.0 & 295.6 & 35.29 \\
\hline \hline
\end{tabular}

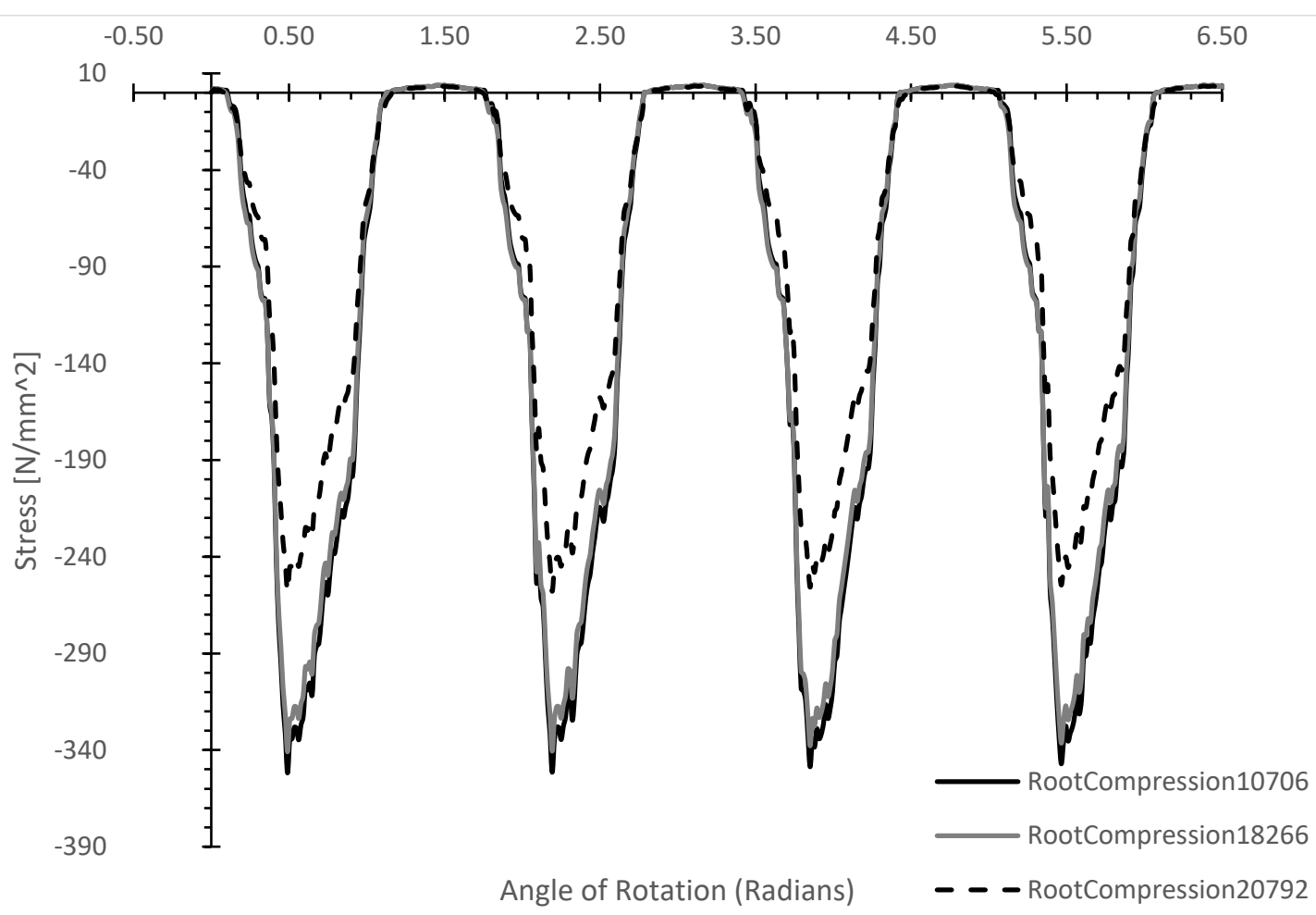

Figure 40. Minimum principal, compressive, stress from the root center to the face 


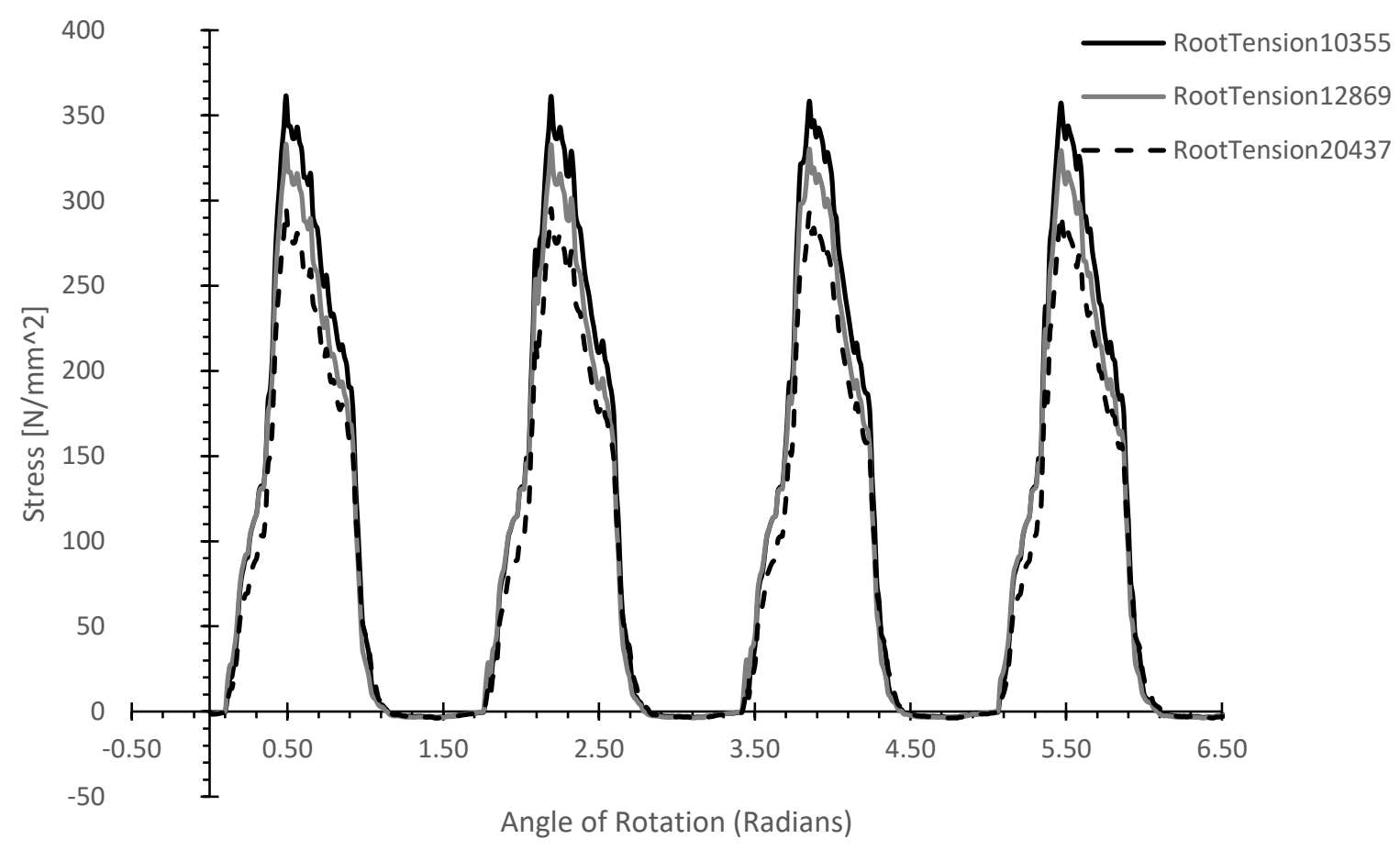

Figure 41.Maximum principal, tensile, stress from the root center to the face Table 13 indicates nodes with their associated stress probe values measure at an instant in time at the point of gear teeth contact. The nodes selected were located at the center of the root (10706, 10355), between the face and the center $(12869,18266)$, and at the edge of the root and the face (20437, 20792). Figures 40 and 41 shows the stress at the roots of the gear tooth as a single tooth rotates around one revolution. The force indicated is the maximum and minimum principle stresses at the root face of the gear, showing root tensile stress and root compression on the opposite side. It can be seen that, the stress increases as the tooth rotates into contact with each planet and stress decreases when moving away from the center of the root. The tensile stress is seen to be slightly greater in magnitude than the compressive stress on the gear root. From the contour plot off the maximum and minimum principle stress at the root can be observed. The maximum principle stress at the root is $364.1 \mathrm{MPa}$ and the minimum principle stress at the 
opposite side is $-362.4 \mathrm{MPa}$. The maximum principle stress and the minimum are shown in Figures 42 and 43.

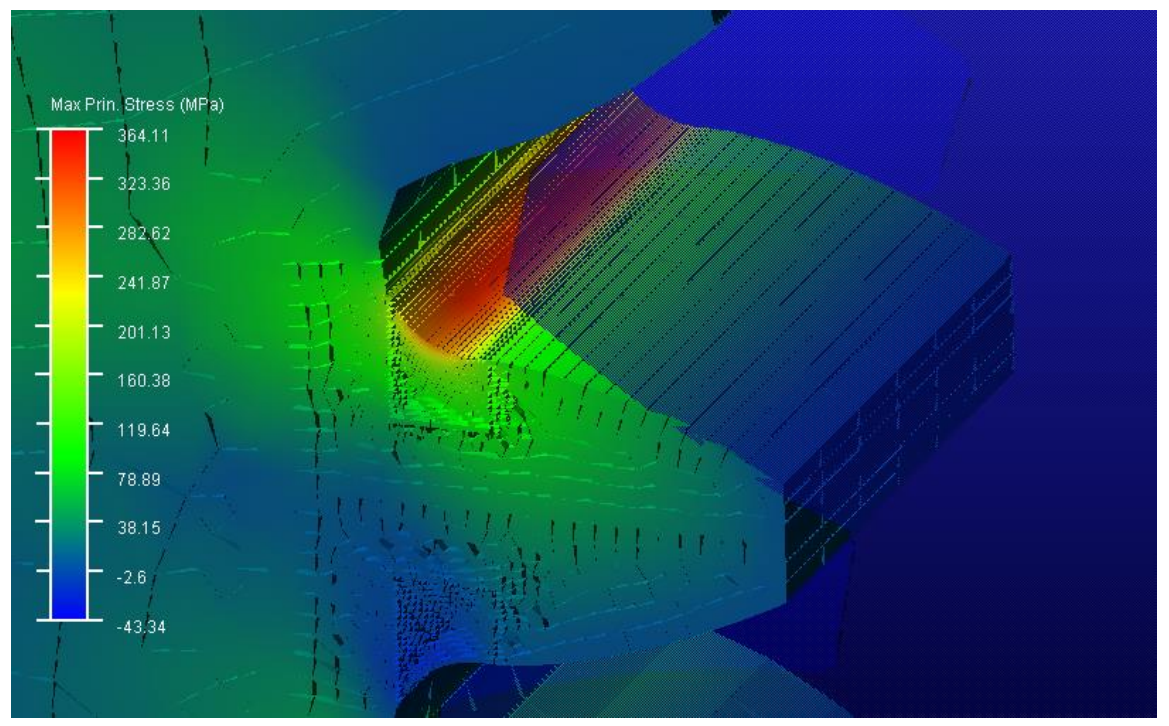

Figure 42. Maximum principal stress contour plo

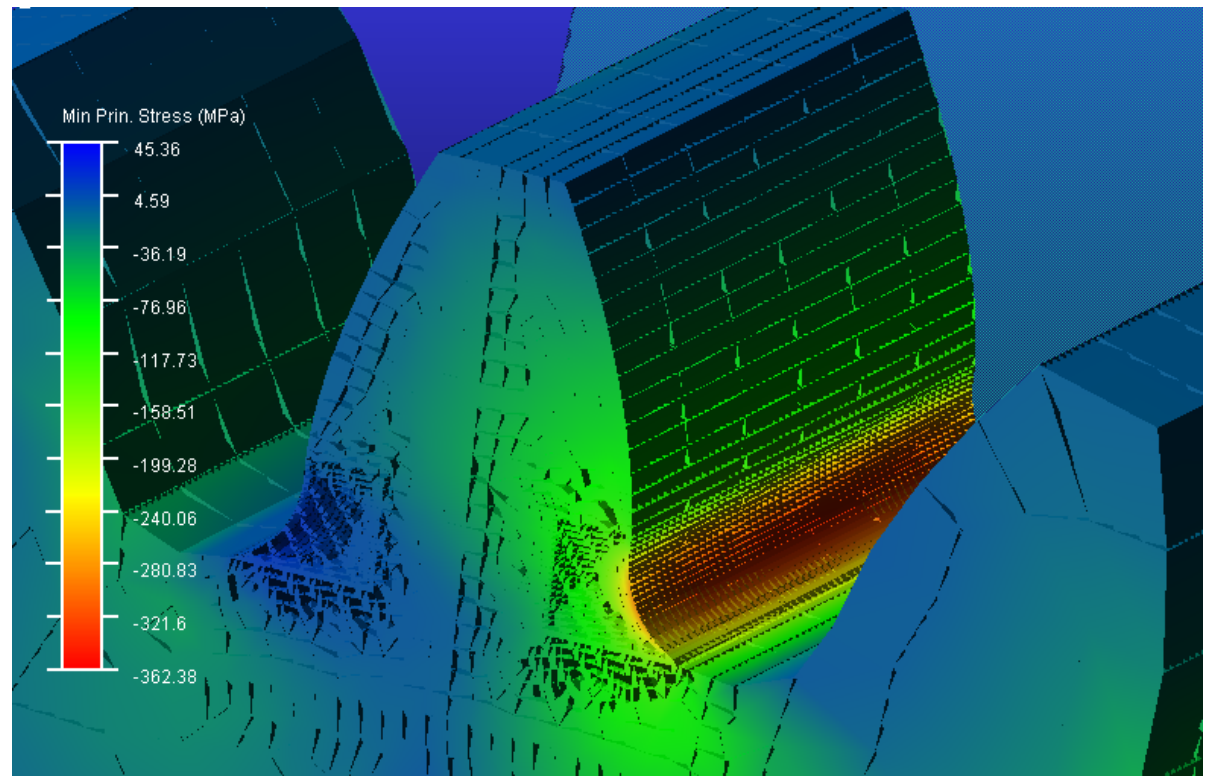

Figure 43. Minimum principal stress contour plot

\subsection{CONTACT FORCE}

The contact force was plotted the series of gear contact locations. These contact locations

includes all contact definition within the Adams model created. The contact force between the 
gear teeth is the dynamic force model by the simulation created in Adams. The advantage of this model allows for the analysis of the system according to what is expected to be the dynamic behavior of the system instead of using a simple static system approximation.

The contact force was compared between a fully rigid model and a model with a flexible body at the sun gear in the first stage. The contact force shows an interesting phenomenon when plotted with a flexible body. The contact force plot is shown in Figure 44.

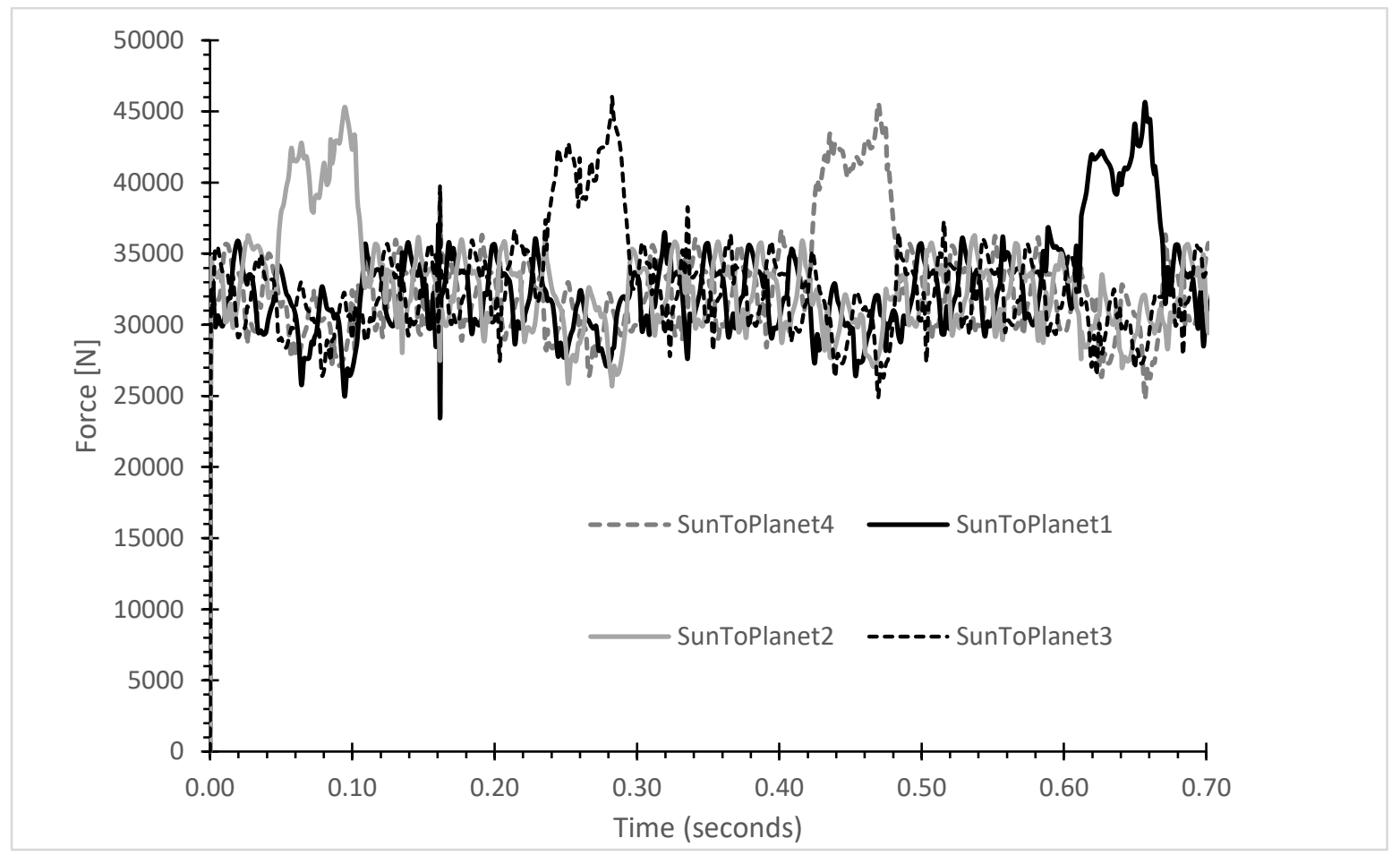

Figure 44. Gear Contact Force Stage 1 Sun to Planets with Flexible Body.

The contact force shown averages around $32.5 \mathrm{kN}$. Figure 44 show is the contact force over one revolution of the sun gear in the first stage with the flexible body. The finite element body affects the contact force between the flexible body and the rigid body. The four peaks shown occur when the finely meshed gear tooth comes into contact with the other rigid planets. As the tooth meshes, the contact force increases between the sun gear and that particular planet but the contact force on the other three mesh location decreases. The overall result creates an average 
force between the four contact force to be the relatively the same within the accuracy of the force measurement. Without a flexible body the force profile is steady and consistent through a full rotation. This is show in Figure 45.

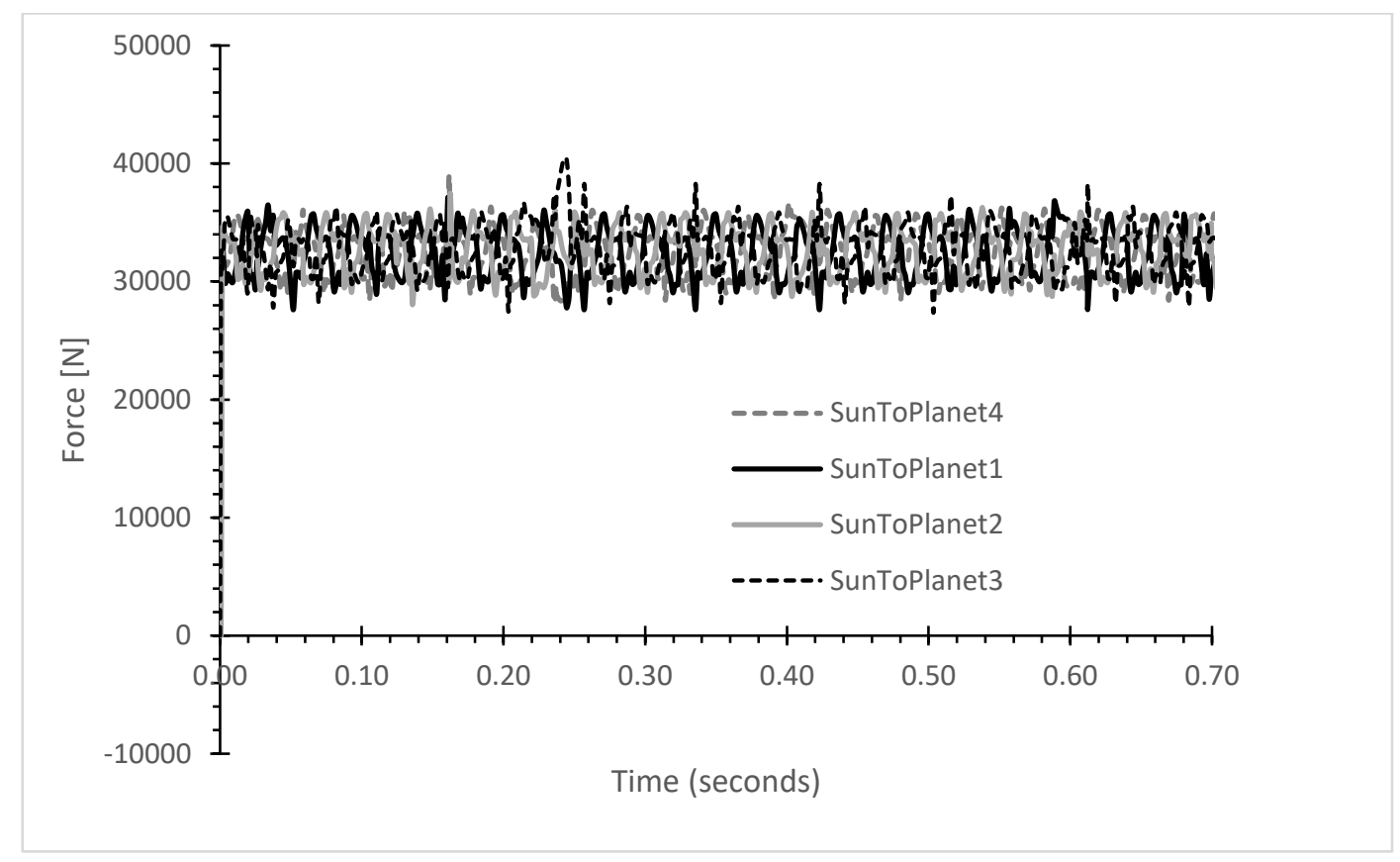

Figure 45. Gear Contact Force Stage 1 Sun to Planets without Flexible Body.

\subsection{FAST FOURIER TRANSFORM}

Fast Fourier Transform, FFT, is a method of signal analysis which filters an input signal and outputs its frequency components. An FFT takes an incoming signal, usually a signal in time, and uses a mathematical algorithm to isolate individual sinusoidal signals and display them in frequency space. A diagram of this action is shown in Figure 46. 


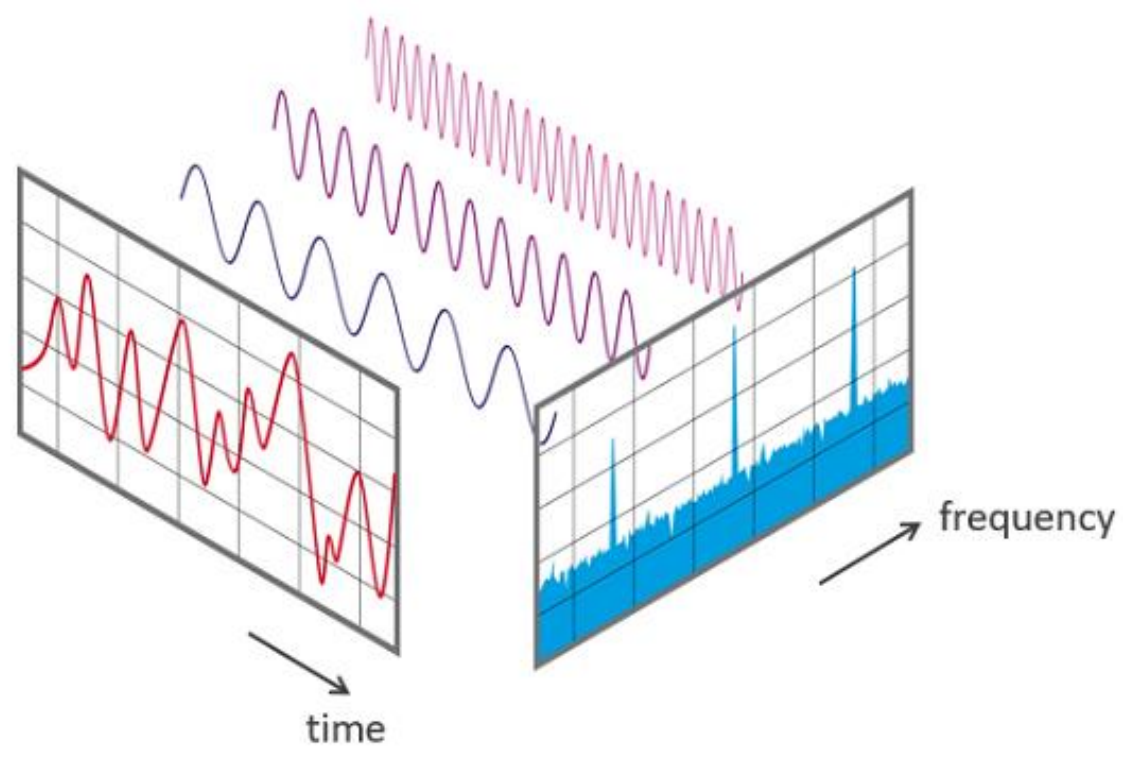

Figure 46. A signal viewed in frequency and time domain [23]

The FFT plot is often used to analyze faults, quality control and condition monitoring of machines and systems [23]. As can be seen in Figure 46, an arbitrary signal with three superimposed sinusoidal waves is measured over a period of time shown in red on the left. These three signals are associated with a specific amplitude and frequency. Using an FFT function on a processing software the three signals are isolated to produce three individual peaks on a frequency versus amplitude plot shown as the plot in blue on the right. Peaks at higher frequencies on a FFT plot can occur at integer multiples of the primary frequency on an FFT, any other frequencies are considered noise or non-periodic behavior.

The usage of FFT in gear health analysis can be significant since gear contact occurs at specific frequencies unique to the gear system designed. The frequencies at which gears mesh is defined as the Gear Mesh Frequency, GMF. The gear mesh frequency can be calculated for each meshing gear pair as the rotational velocity multiplied by the number of teeth of the gear for a standard fix axis gear mesh pair. For a planetary gear system, the GMF is defined by the carrier 
speed multiplied by the number of teeth on the ring gear. There is one specific GMF for each gear contact which can be calculated from either the pinion or the gear in a fixed axis gear mesh. In the simulations used for the following analyses, the FFT plot is created using the contact force between the gears a specified time period. The FFT is used to distinguish a unique vibration pattern for the planetary gearbox. Frequencies are expected to develop at each specific GMF or an integer multiple of the GMF, $n *$ GMF. A realistic measurement of gearbox vibration frequency is to place an accelerometer at each stage on the housing of the system. This allows for a measurement of the acceleration of the system over time which can then be input to generate an FFT since measure the contact force directly in a planetary gear system would not be practical. Gearbox vibration frequency FFTs can indicate defects in the system through a difference in amplitudes, modulation of the primary GMF, or, but not limited to, the occurrence of subharmonics/super-harmonic frequency peaks. Each different frequency occurrence is associated with a particular defect which can include, but are not limited to, bearing defect, gear eccentricity, shaft defect, or gear teeth defect.

\subsection{VIBRATION ANALYSIS THROUGH FFT}

The first model to be observed is a first stage model with rigid bodies. The model was simulated for one quarter turn on the input over 2000 steps with an input speed of $1.76 \mathrm{rad} / \mathrm{sec}$. This input speed corresponds to $16.8 \mathrm{RPM}$ which is remains on the high-side of the N90's operating range during its low speed operations. This generates over one full revolution on the sun gear output. The output force was plotted and used to generate the FFT for force in the frequency domain. This plot is shown as Figure 47 . 


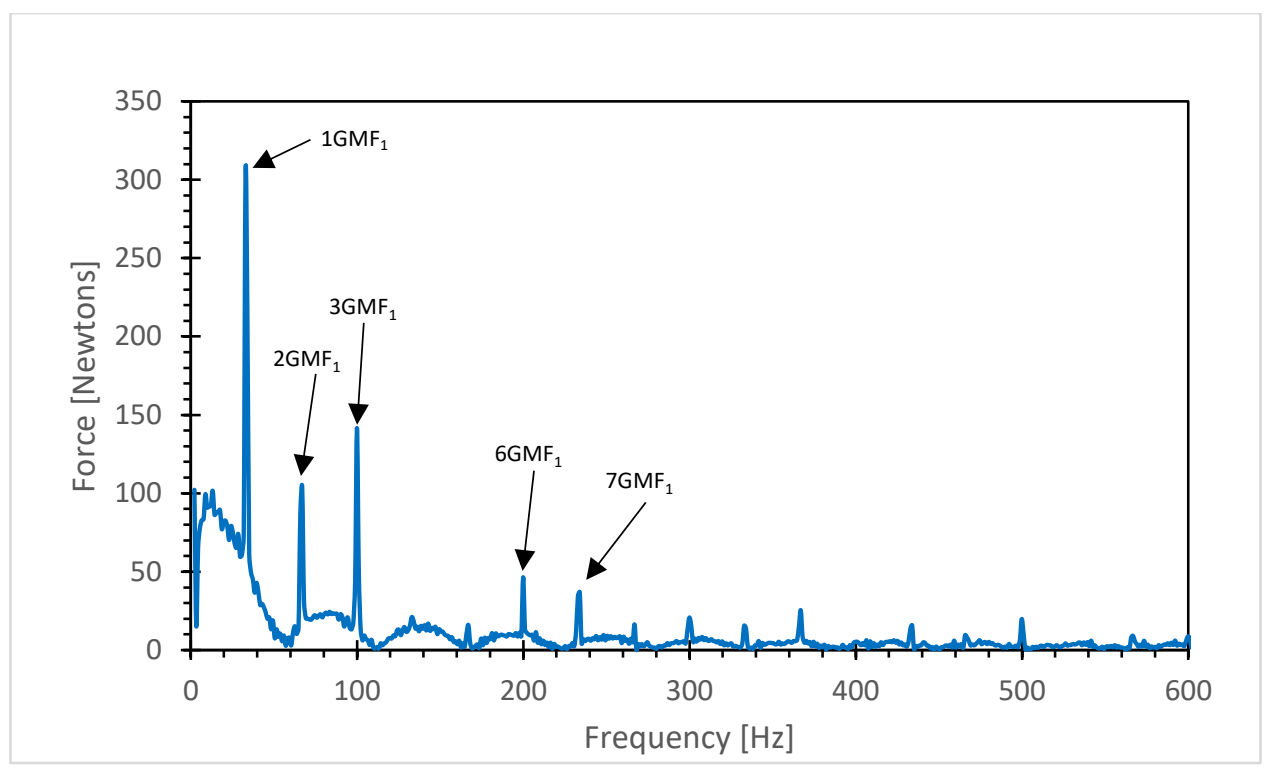

Figure 47. Contact force FFT sun to planet Stage 1

It can be seen in Figure 47 that the FFT exhibits peaks at very specific locations throughout the frequency band ranging from 0 to $600 \mathrm{~Hz}$. The larges peak occurs that the expected GMF frequency of $33.3 \mathrm{~Hz}$. This is calculated from the carrier input speed of $1.76 \mathrm{rad} / \mathrm{sec}$ multiplied by the number of teeth on the ring gear, 119 teeth, for a planetary gear system. All remained frequencies occur are super-harmonic frequencies of $\mathrm{GMF}(2 * \mathrm{GMF}, 3 * \mathrm{GMF} \ldots)$. A peak amplitude table is listed in Table 14 of the FFT.

Table 14. FFT Stage 1 contact force between sun and planet gear frequency amplitudes

\begin{tabular}{crr}
$\begin{array}{c}\text { Frequency } \\
\text { [Hz] }\end{array}$ & $\begin{array}{c}\text { Amplitude } \\
\text { [Newton] }\end{array}$ & $\mathrm{n}^{*} \mathrm{GMF}$ \\
\hline 32.96 & 305.29 & 1 \\
67.01 & 104.2 & 2 \\
99.97 & 141.62 & 3 \\
134.02 & 18.71 & 4 \\
166.98 & 15.98 & 5 \\
199.94 & 46.46 & 6 \\
232.90 & 34.31 & 7 \\
266.95 & 16.42 & 8 \\
\hline
\end{tabular}


The frequencies demonstrate the vibration pattern generated by such a system at the first stage of the gearbox. Figure 47 contains points which indicate peaks and its associated GMF harmonic which corresponds to values in Table 14 .

A defect is applied to one of the teeth at the stage one sun gear. The sun gear has been known to be the most common source of defect occurrence in wind turbine gearboxes. The defect is imposed at $25 \%$ of the root thickness. The same simulation is conducted. The contact force between the sun gear and the planet gear is taken to generate an FFT plot.

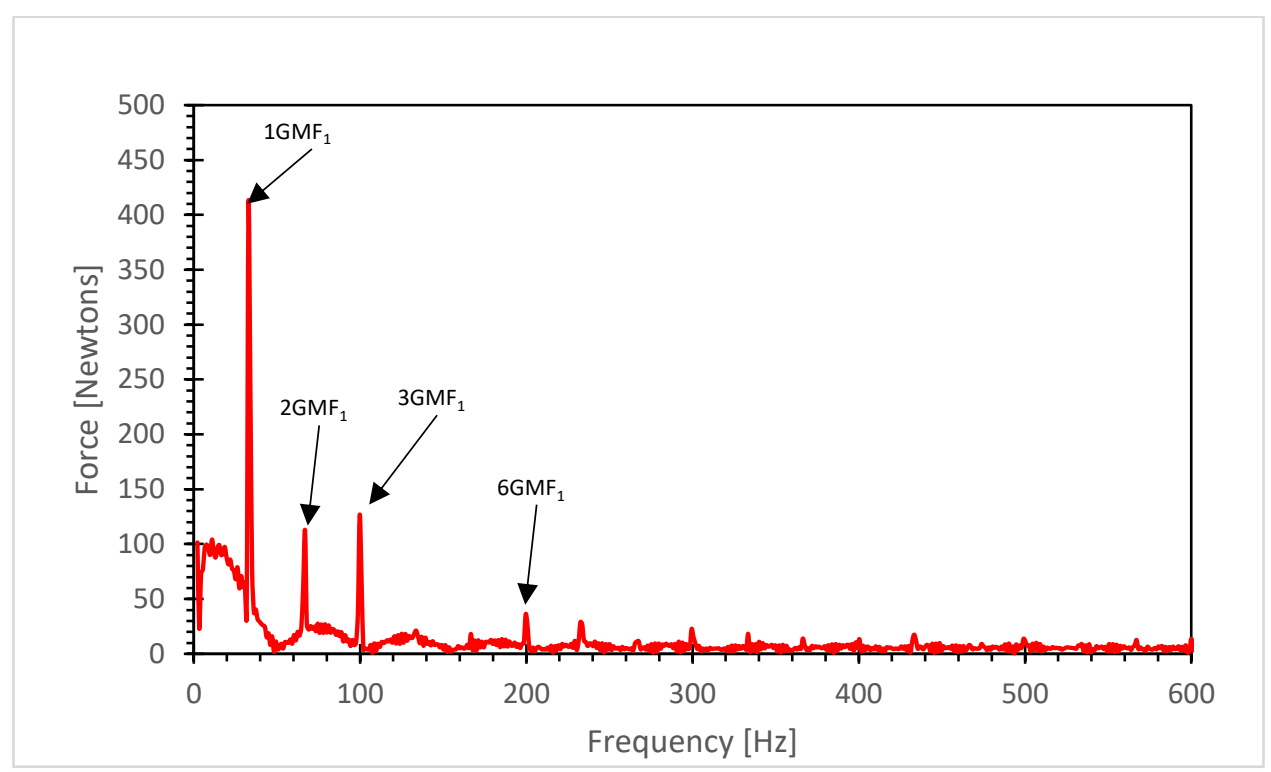

Figure 48. Stage 1 contact force FFT between sun and planet gears with a defect on the sun gear tooth. 


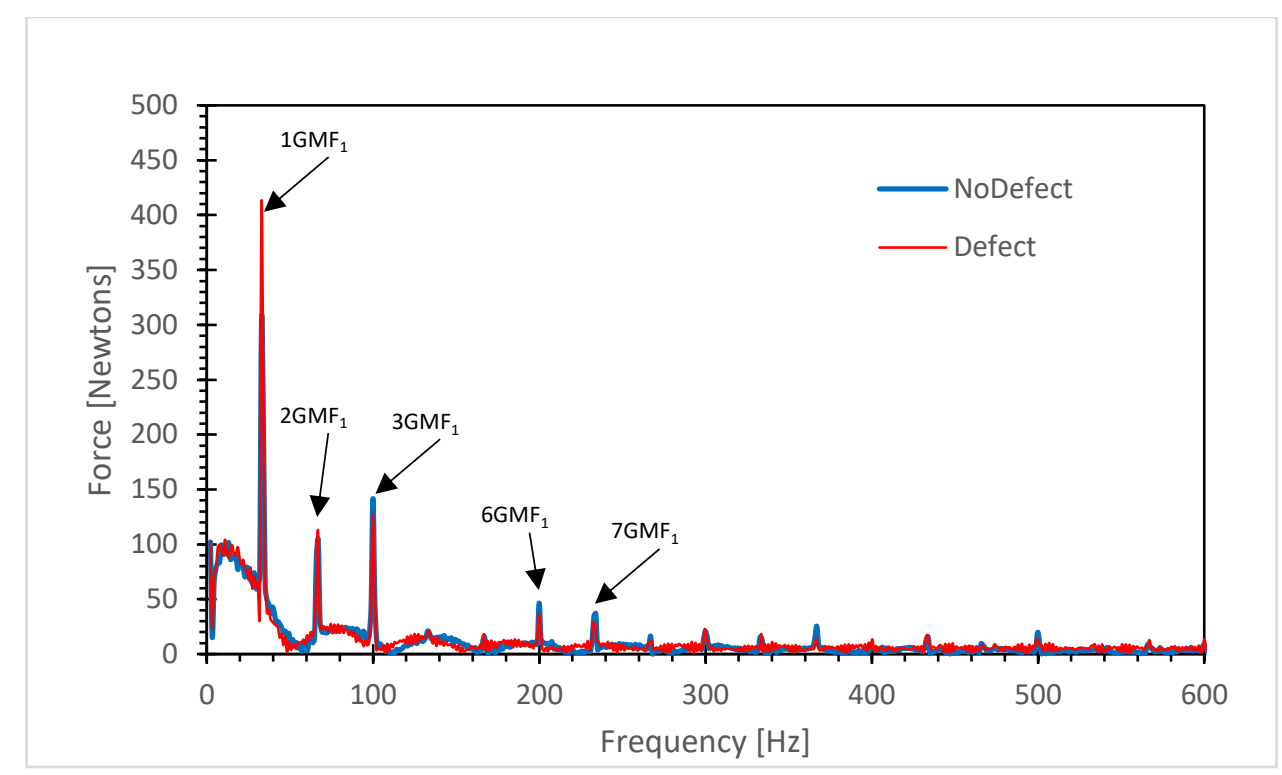

Figure 49. Stage 1 superposition comparison FFT of the contact force between the sun and planet gear with and without a defect at the sun gear tooth.

Figure 48 shows the vibration pattern generated by the first stage model with a defect at the first stage sun gear, points are present to indicate the GMF peaks which are present in the pattern.

Figure 49 represents the superposition of the vibration pattern for the first stage with and without a defect that the sun gear tooth. The healthy gear FFT is represented by the blue curve and the defective tooth FFT is represented by the red curve. Table 15 shows the amplitudes of each FFT plot at the various frequency peaks.

Table 15. Comparison between amplitudes at the GMF harmonics of Stage 1 with and without a defect at the sun gear tooth.

\begin{tabular}{crrrr}
$\begin{array}{c}\text { Frequency } \\
{[\mathrm{Hz}]}\end{array}$ & GMF & \multicolumn{2}{c}{ Amp [N] } & \% Change \\
\hline 32.96 & 1 & 305.29 & 408.14 & 33.69 \\
67.01 & 2 & 104.2 & 112.57 & 8.03 \\
99.97 & 3 & 141.62 & 126.59 & -10.61 \\
134.02 & 4 & 18.71 & 21.12 & 12.88 \\
166.98 & 5 & 15.98 & 18.19 & 13.83 \\
199.94 & 6 & 46.46 & 35.98 & -22.56 \\
232.9 & 7 & 34.31 & 26.89 & -21.63 \\
\hline
\end{tabular}


From both Figure 49 and Table 15, it is shown that there are changes in amplitude at each GMF on between the healthy and defective models. The most significant impact that can be seen visually in Figure 49 corresponds to the amplitude change at the primary GMF, 33Hz. This is the location of the largest peak as well as a $33.7 \%$ change in amplitude between the two models. Although, there are other peaks which exhibit a large amplitude difference, the overall magnitude of the change is quite small compared to the change at the first GMF.

A similar analysis was performed on the models with a flexible body present as the sun gear with both healthy and defective tooth. The exact same base model was used, and the meshing was kept consistent between the two models, however as with different geometry when there is a defect present versus no defect the meshed model cannot be made the same. The comparison is show in Figure 50.

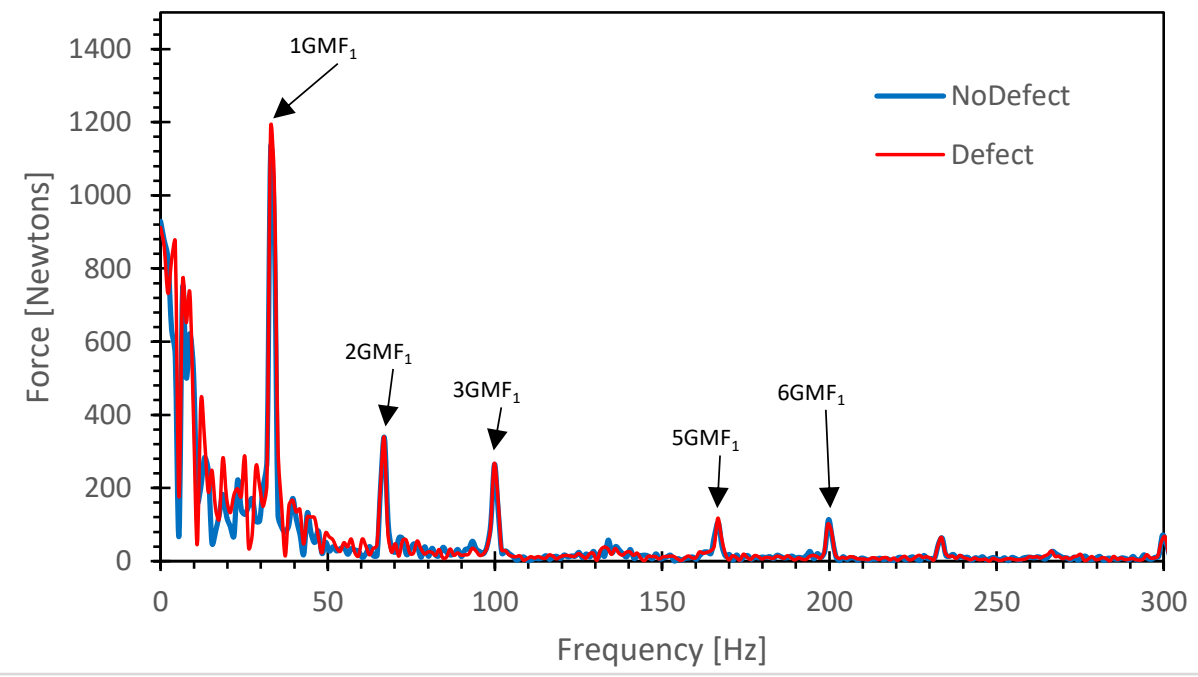

Figure 50. Stage 1 superposition comparison FFT of the contact force between the sun and planet gear with and without a defect at the sun gear tooth using flexible bodies. 
Table 16. Comparison between amplitudes at the GMF harmonics of Stage 1 with and without a defect at the sun gear tooth using flexible bodies.

\begin{tabular}{crrrr}
$\begin{array}{c}\text { Frequency } \\
{[\mathrm{Hz}]}\end{array}$ & GMF & \multicolumn{2}{c}{ Amp $[\mathrm{N}]$} & $\%$ \\
\hline 32.96 & 1 & 1113.51 & 1176.7 & 5.67 \\
66.95 & 2 & 336.87 & 337.12 & 0.07 \\
99.87 & 3 & 264.57 & 265.84 & 0.48 \\
166.98 & 5 & 105.03 & 117.44 & 11.82 \\
199.94 & 6 & 113.09 & 100.65 & -11.00 \\
\hline
\end{tabular}

A similar trend is presented in Figure 50 and Table 16. It is seen that there is a noticeable amplitude change at the primary GMF frequency. The presence of a defect induces a change in the vibration as can be seen in the FFT of a noticeable magnitude change primarily at the first GMF for a single stage planetary gear system. In the case of the just analyzing the first stage of the planetary system by itself, the differences between a healthy and defective gearbox presents itself through a change in amplitude.

\subsection{MULTISTAGE ANALYSIS}

The FFT analysis was performed on the system as a whole, involving the three stages put together as a full assembly. The full system analysis is expected to provide a more realistic representation of such a system as the N90. This system was simulated with a carrier speed of $1.76 \mathrm{rad} / \mathrm{sec}(16.8 \mathrm{RPM})$. 


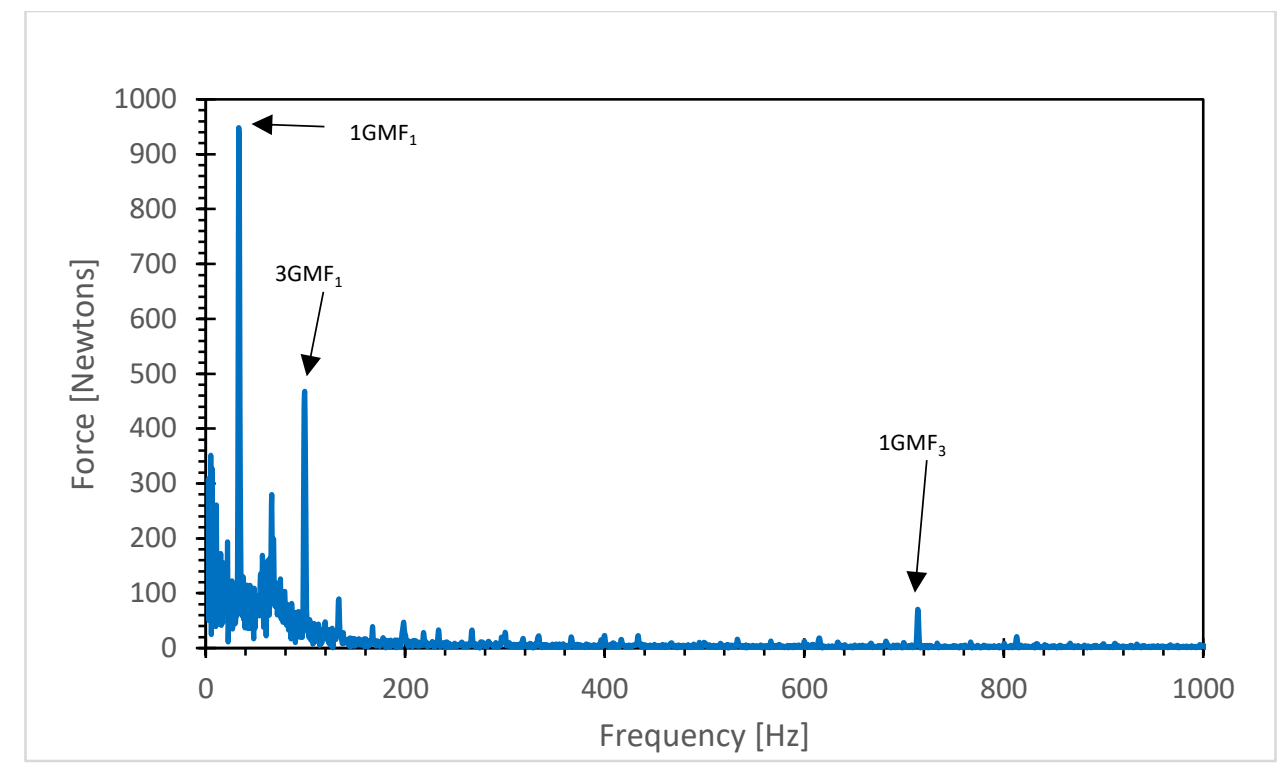

Figure 51. FFT of Stage 1 contact force between sun and planet gear in a full three stage assembly.

In Figure 51, the contact force at the first stage sun gear was plotted to generate a FFT. The FFT was generated based on a fully assembled model of the planetary gearbox. The frequencies present indicated the GMF at the first stage and other super-harmonics with lower amplitude peaks. However, at $714 \mathrm{~Hz}$, there is an amplitude peak present. This peak represents the $\mathrm{GMF}_{3}$ which is the output to the generator. 


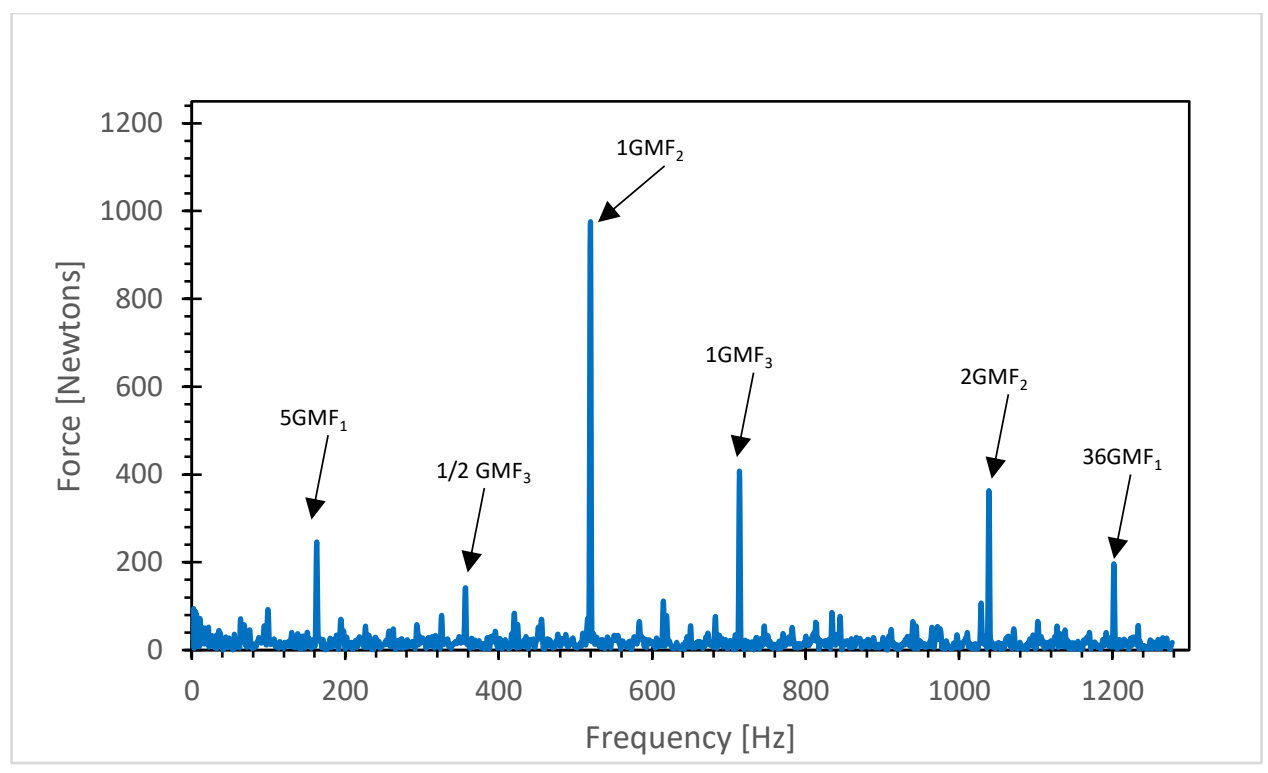

Figure 52. FFT of Stage 2 contact force between sun and planet gear in a full three stage assembly.

The second stage FFT, Figure 52, exhibits multiple peaks at a variety of frequencies not unique to its own stage. The frequency spectrum is dominated by $\mathrm{GMF}_{2}$ however harmonics of $\mathrm{GMF}_{1}$ and $\mathrm{GMF}_{3}$ are represented in the spectrum. The pointers in Figure 52 shows the representations of these GMF's.

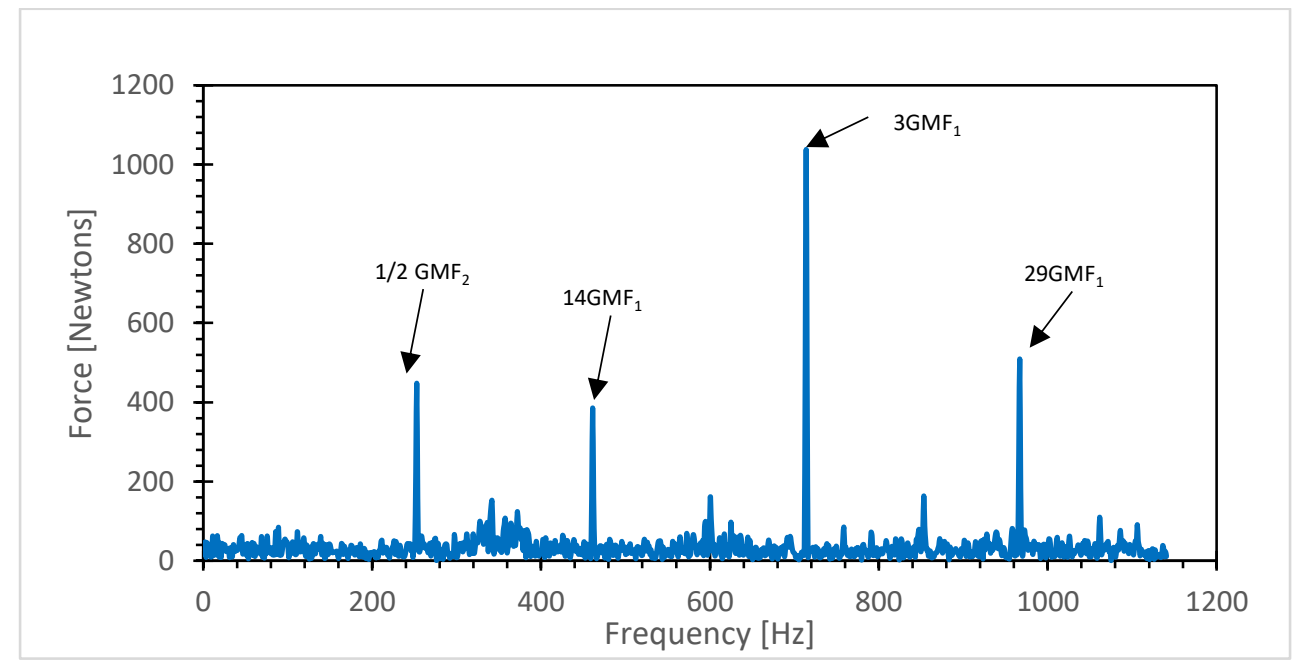

Figure 53. FFT of Stage 3 contact force between sun and planet gear in a full three stage assembly. 
Figure 53 shows the FFT produced from the contact force at stage three output. An interesting pattern can be seen in this plot as there are peaks from harmonic frequencies from the other two stages. All three stages are represented in this FFT by harmonics of their respective GMF's illustrated by the pointers in Figure 53. As seen previously in the other stages, the FFT is dominated by the $\mathrm{GMF}_{3}$.

For further vibration analysis of the full system gearbox, a defect was imposed at the sun gear in the first stage. The system model was simulated. At each stage the contact force was plotted to generate an FFT representing the vibration pattern at each stage. It is expected that there will be the existence of additional frequencies in each stage not present if each were to be analyzed separately. This shows the complex nature of the gear system where each stage can influence the behavior in the other stages.

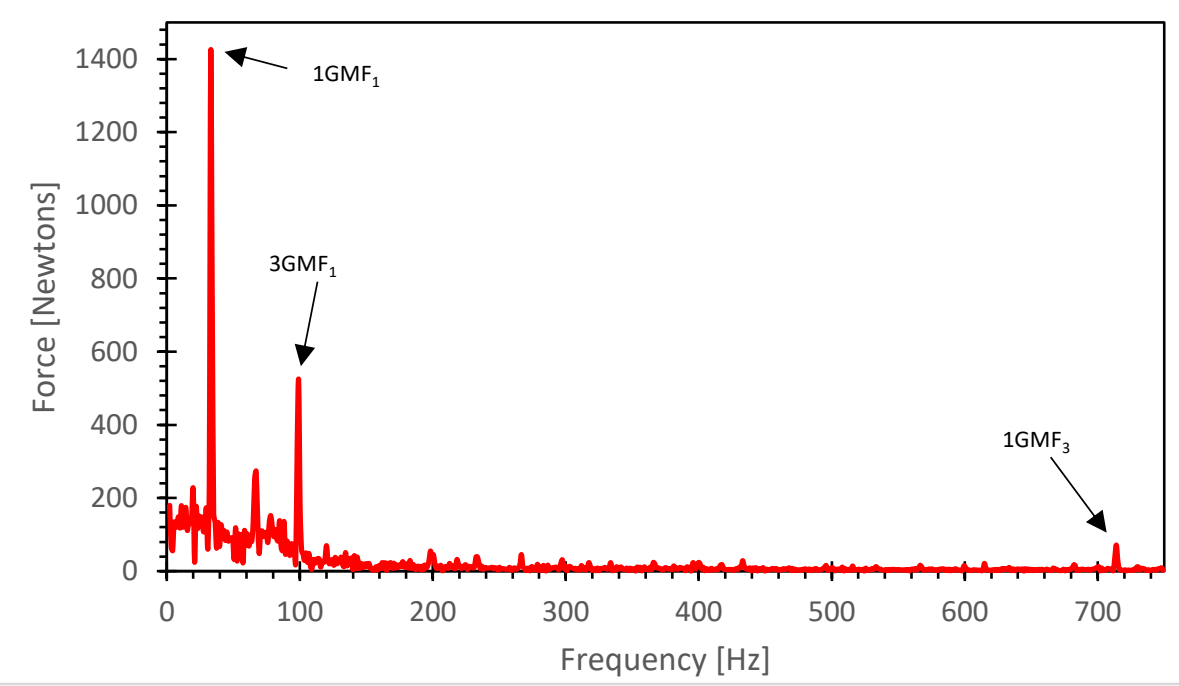

Figure 54. FFT of Stage 1 contact force between sun and planet gear in a full three stage assembly with a defect at first stage sun gear. 


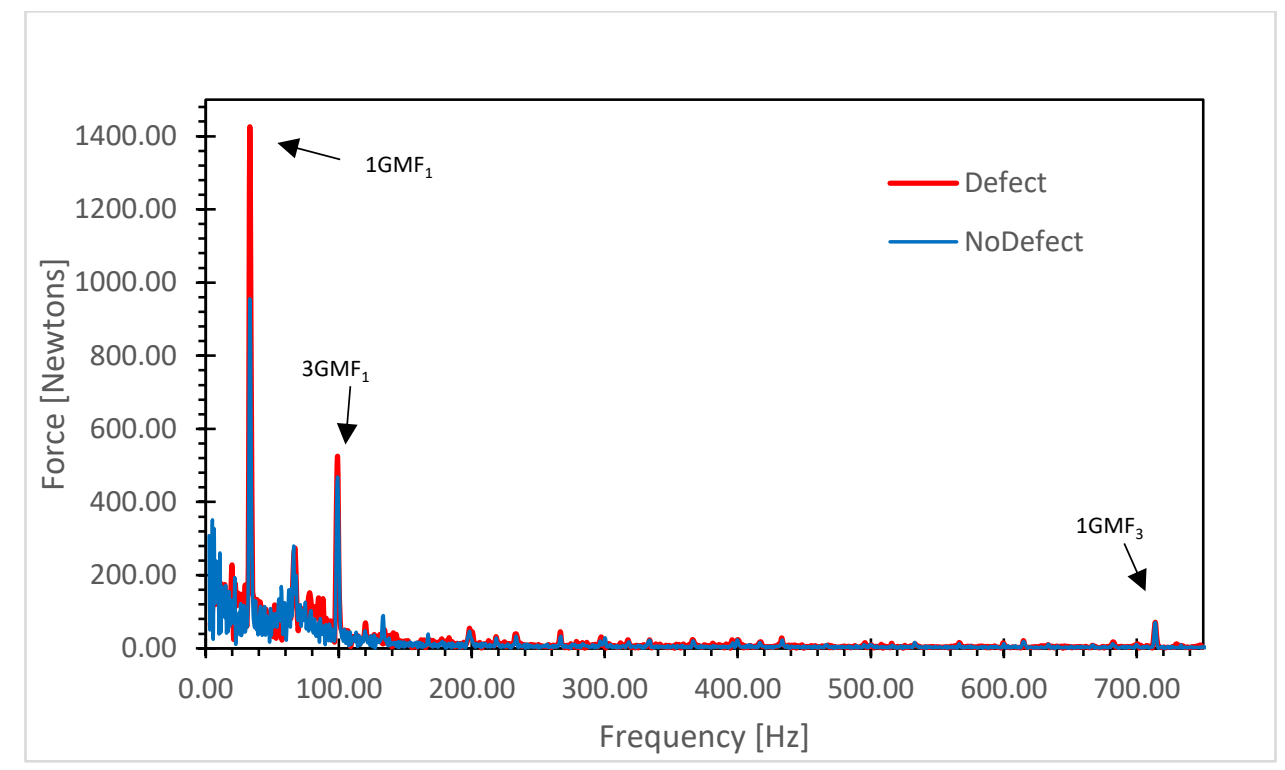

Figure 55. Stage 1 superposition comparison FFT of the contact force between the sun and planet gear with and without a defect at the sun gear tooth.

Figure 55 shows the FFT plot for the contact force at the first stage of the gearbox. Similarly to the isolated analysis of the first stage, it is seen that the presence of a defect appears to only affect the magnitude of the peaks in the FFT. The comparison between the stages with no defect versus a defect at the sun gear can be seen at primarily at the $\mathrm{GMF}_{1}$. Table 17 compares the amplitudes at each frequency.

Table 17. Comparison between amplitudes at the GMF harmonics of Stage 1 with and without a defect at the sun gear tooth.

\begin{tabular}{ccrrr}
$\begin{array}{c}\text { Frequency } \\
{[\mathrm{Hz}]}\end{array}$ & GMF & \multicolumn{2}{c}{ Amp $[\mathrm{N}]$} & $\%$ \\
\hline 32.96 & $1 * \mathrm{GMF}_{1}$ & 948.5 & 1415.4 & 49.23 \\
99.87 & $3 * \mathrm{GMF}_{1}$ & 442.4 & 521.2 & 17.81 \\
714.4 & $1 * \mathrm{GMF}_{3}$ & 60.43 & 71.44 & 18.22 \\
\hline
\end{tabular}

The two changes observed are at $33 \mathrm{~Hz}$ and $99 \mathrm{~Hz}$. This represents behavior at the first stage as a result of the crack at the first stage sun gear. The other frequency present is the $\mathrm{GMF}_{3}$ seen at 
$714 \mathrm{~Hz}$. It is not expected that there be significant changes to the amplitude at $714 \mathrm{~Hz}$. However, if changes to the third stage GMF were to occur it is unlikely to be seen as significant compared to the GMF and 3GMF at Stage 1.

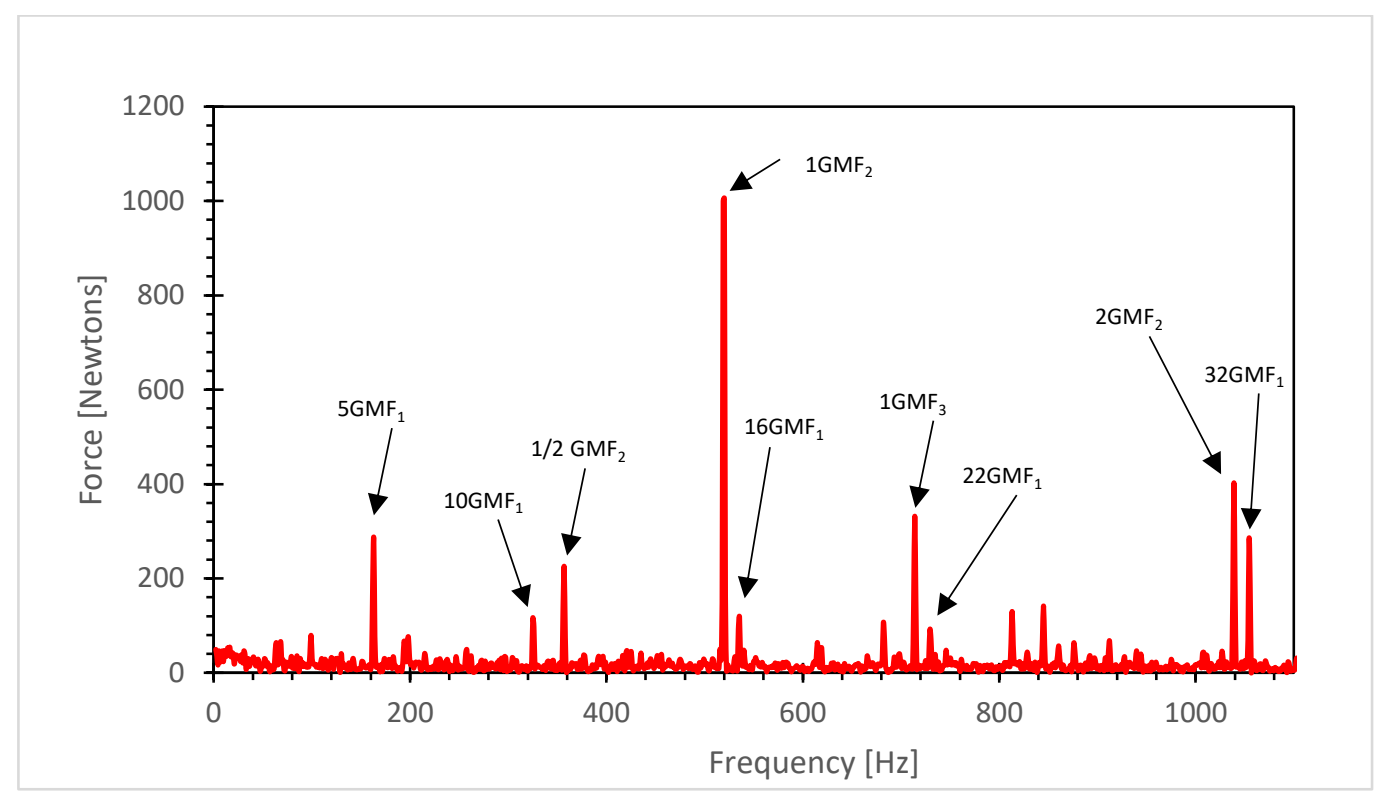

Figure 56. FFT of Stage 2 contact force between sun and planet gear in a full three stage assembly with a defect at first stage sun gear. 


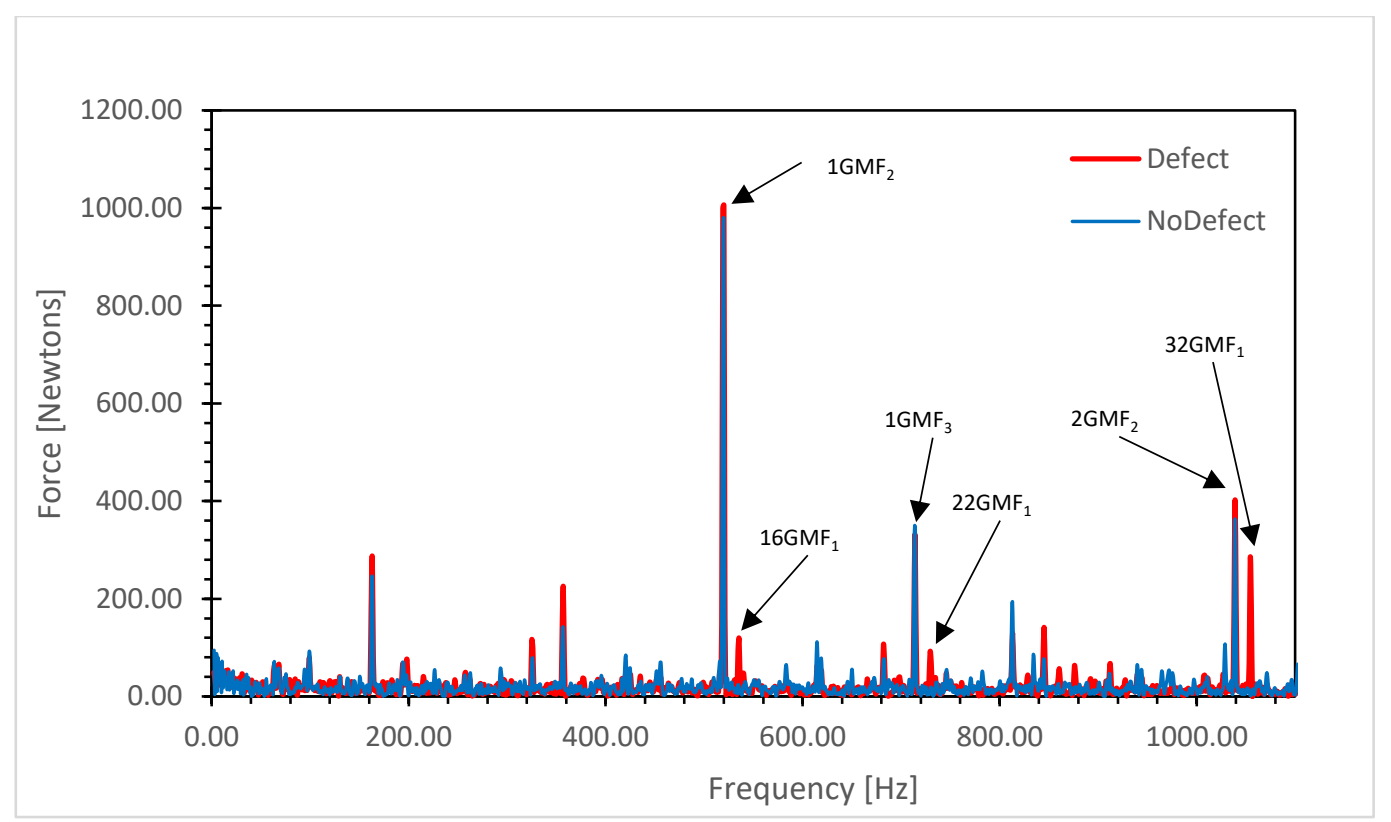

Figure 57. Stage 2 superposition comparison FFT of the contact force between the sun and planet gear with and without a defect at the sun gear tooth.

Figure 56 shows the FFT plot generated from the contact force at stage two between the sun gear and the planet gear with a defect at the first stage sun gear. Figure 57 shows the superposition of the FFT with no defect (blue) and a defect at the first stage sun gear (red). Pointers in Figure 57 highlight appearances of certain frequencies in the defective gearbox not seen in the nondefective assembly. In Figure 57, the three indicated peaks representing harmonics of $\mathrm{GMF}_{2}$ and $\mathrm{GMF}_{3}$ are modulated by higher harmonics of $\mathrm{GMF}_{1}$. The frequency difference is expressed in Table 18.

Table 18. Comparison between amplitudes at the GMF harmonics of Stage 2 with and without a defect at the sun gear tooth.

\begin{tabular}{rcrrr}
$\begin{array}{c}\text { Frequency } \\
{[\mathrm{Hz}]}\end{array}$ & GMF & \multicolumn{2}{c}{ Amp [N] } & $\%$ \\
\hline 519.8 & $1 \mathrm{GMF}_{2}$ & 925.7 & 1005.6 & 8.63 \\
535.0 & $16 \mathrm{GMF}_{1}$ & 15.1 & 118.3 & 683.4 \\
714.1 & $\mathrm{CGMF}_{3}$ & 350.1 & 331.7 & -5.26 \\
732.4 & $22 \mathrm{GMF}_{1}$ & 18.78 & 91.6 & 387.5 \\
1038.0 & $2 \mathrm{GMF}_{2}$ & 363.2 & 402.5 & 10.8 \\
1064.4 & $32 \mathrm{GMF}_{1}$ & 9.43 & 283.3 & 2904 \\
\hline
\end{tabular}


Table 18 shows the three large peaks of $\mathrm{GMF}_{2}$ and $\mathrm{GMF}_{3}$ modulated by $\mathrm{GMF}_{1}$ harmonics. In this case, it can be suspected that this maybe the indication of a vibration signature of a defect at the first stage sun gear represented through the second stage FFT analysis, the development of a vibration pattern will be discussed further in the next section.

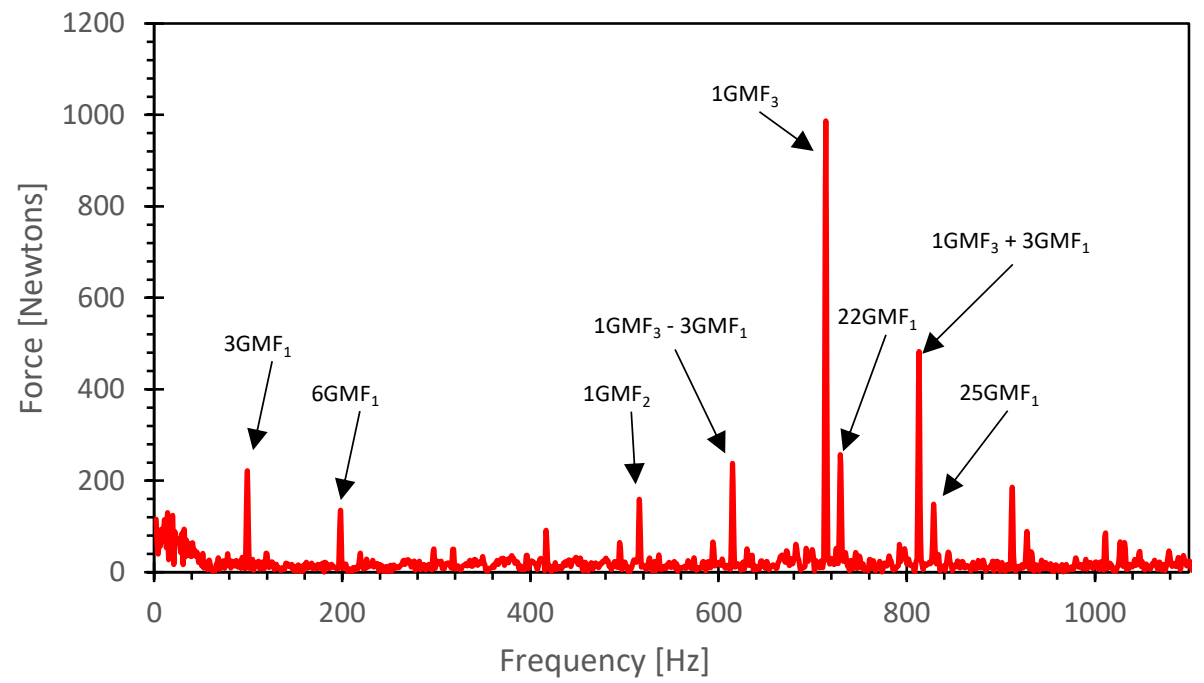

Figure 58. FFT of Stage 3 contact force between sun and planet gear in a full three stage assembly with a defect at first stage sun gear.

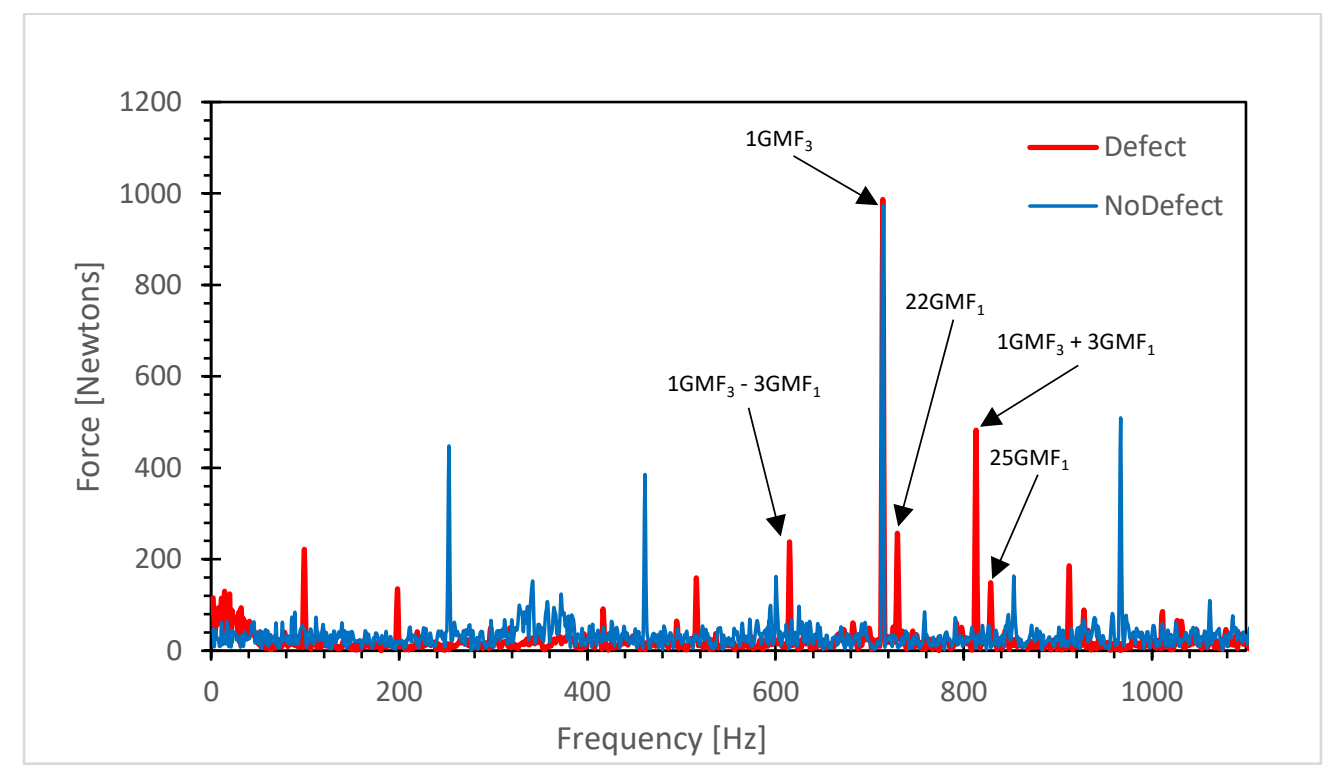


Figure 59. Stage 3 superposition comparison FFT of the contact force between the sun and planet gear with and without a defect at the sun gear tooth.

Figure 58 shows the FFT vibration pattern for the third stage with a defect at the first stage sun gear. Figure 59 shows the superposition of the FFT from both a defective and a non-defective gearbox. The third stage FFT shows significant changes in the frequency patterns between the two gearboxes. Figure 58 shows the variation of GMF's between all three stages which are present in the FFT. Figure 59 highlights the significant occurrences of the frequencies which appear due to the defect. It can be seen that sideband frequencies appear at both a higher and lower frequency to the dominant $\mathrm{GMF}_{3}$. Sideband frequencies occur at $1 \mathrm{GMF}_{3}-3 \mathrm{GMF}_{1}$ and $1 \mathrm{GMF}_{3}+3 \mathrm{GMF}_{1}$. Along with the sideband frequencies, modulation of these $\mathrm{GMF}_{3}$ and the sidebands by harmonics of the $\mathrm{GMF}_{1}$ can be seen. It can also be noted that the sideband frequency of $1 \mathrm{GMF}_{3}$ occurs at $1 \mathrm{GMF}_{3}$ plus the second dominant frequency, $3 \mathrm{GMF}_{1}$, seen at the first stage FFT. These significant GMF are presented in Table 19.

Table 19. Comparison between amplitudes at the GMF harmonics of Stage 3 with and without a defect at the sun gear tooth

\begin{tabular}{ccrrr}
$\begin{array}{c}\text { Frequency } \\
{[\mathrm{Hz}]}\end{array}$ & \multicolumn{1}{c}{ GMF } & \multicolumn{2}{c}{ Amp [N] } & \multicolumn{1}{c}{$\%$} \\
\hline 614.8 & Healthy $\mathrm{GMF}_{3}-3 \mathrm{GMF}_{1}$ & 48.55 & 221.9 & 357.1 \\
714.1 & $1 * \mathrm{GMF}_{3}$ & 974.3 & 987.6 & 1.37 \\
731.4 & $22 * \mathrm{GMF}_{1}$ & 27.3 & 257.1 & 541.8 \\
& $1 \mathrm{GMF}_{3}+$ & & & \\
813.2 & $3 \mathrm{GMF}_{1}$ & 18.31 & 482.6 & 2530 \\
831.6 & $25^{*} \mathrm{GMF}_{1}$ & 46.9 & 628.6 & 1667 \\
\hline
\end{tabular}




\subsection{VARYING INPUT SPEED VIBRATION TESTS}

The first set of simulations in Adams were performed at steady state conditions using an input velocity at the first stage of $1.76 \mathrm{rad} / \mathrm{sec}$. The same analysis was performed on the model at two different speeds above and below $1.76 \mathrm{rad} / \mathrm{sec}$. The speeds chosen were $1.5 \mathrm{rad} / \mathrm{sec}$ and 2.0 $\mathrm{rad} / \mathrm{sec}$. As a result of the change of input speed the GMF of each system will change accordingly the expected base GMF for both systems are shown in Table 20.

Table 20. Base Gear Mesh Frequencies for different speed input

\begin{tabular}{cccc} 
Speed & \multicolumn{3}{c}{$\begin{array}{c}\text { GMF } \\
{[\mathrm{Hz}]}\end{array}$} \\
{$[\mathrm{rad} / \mathrm{sec}]$} & $\mathrm{S} 1$ & $\mathrm{~S} 2$ & $\mathrm{~S} 3$ \\
\hline 1.5 & 28.4 & 443 & 608 \\
2 & 37.9 & 590 & 811 \\
\hline
\end{tabular}

The speed variation is performed so that the vibration signature pattern can be determined for a defect at the first stage sun gear. The speeds selected for these two tests are within the range of operation of the N90. A speed of $1.50 \mathrm{rad} / \mathrm{sec}$ is equivalent to $14.3 \mathrm{RPM}$, this is in the mid operating range of the N90. An input speed of $2.0 \mathrm{rad} / \mathrm{sec}$ corresponds to $19.1 \mathrm{RMP}$ which is with in one the high end of the operating range during high speed operations of the N90.

The following figures, Figure 60, Figure 61 and Figure 62 show the FFT for at Stage 1 of the contact force between the sun and the planet gear with and without a defect at the first stage sun gear tooth for an input speed of $1.5 \mathrm{rad} / \mathrm{sec}(14.3 \mathrm{RPM})$. 


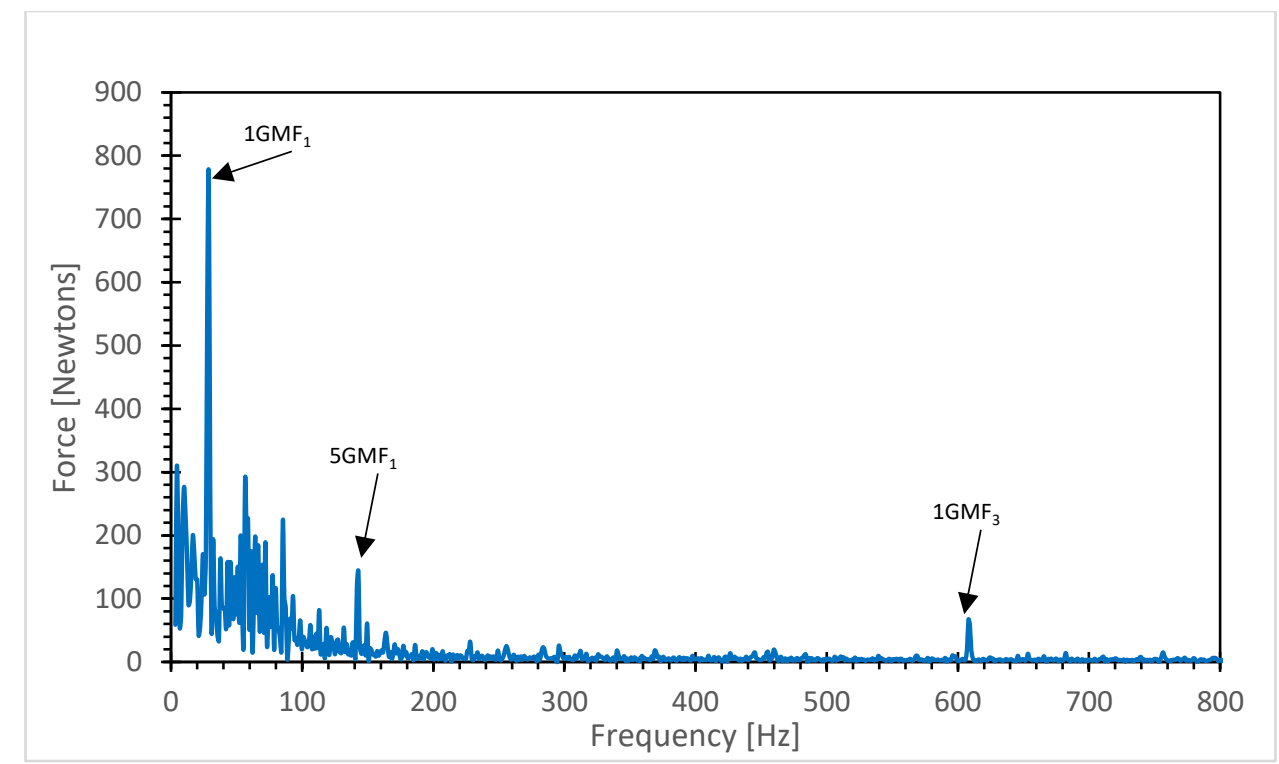

Figure 60. FFT of Stage 1 contact force between sun and planet gear in a full three stage assembly with input speed $1.5 \mathrm{rad} / \mathrm{sec}$.

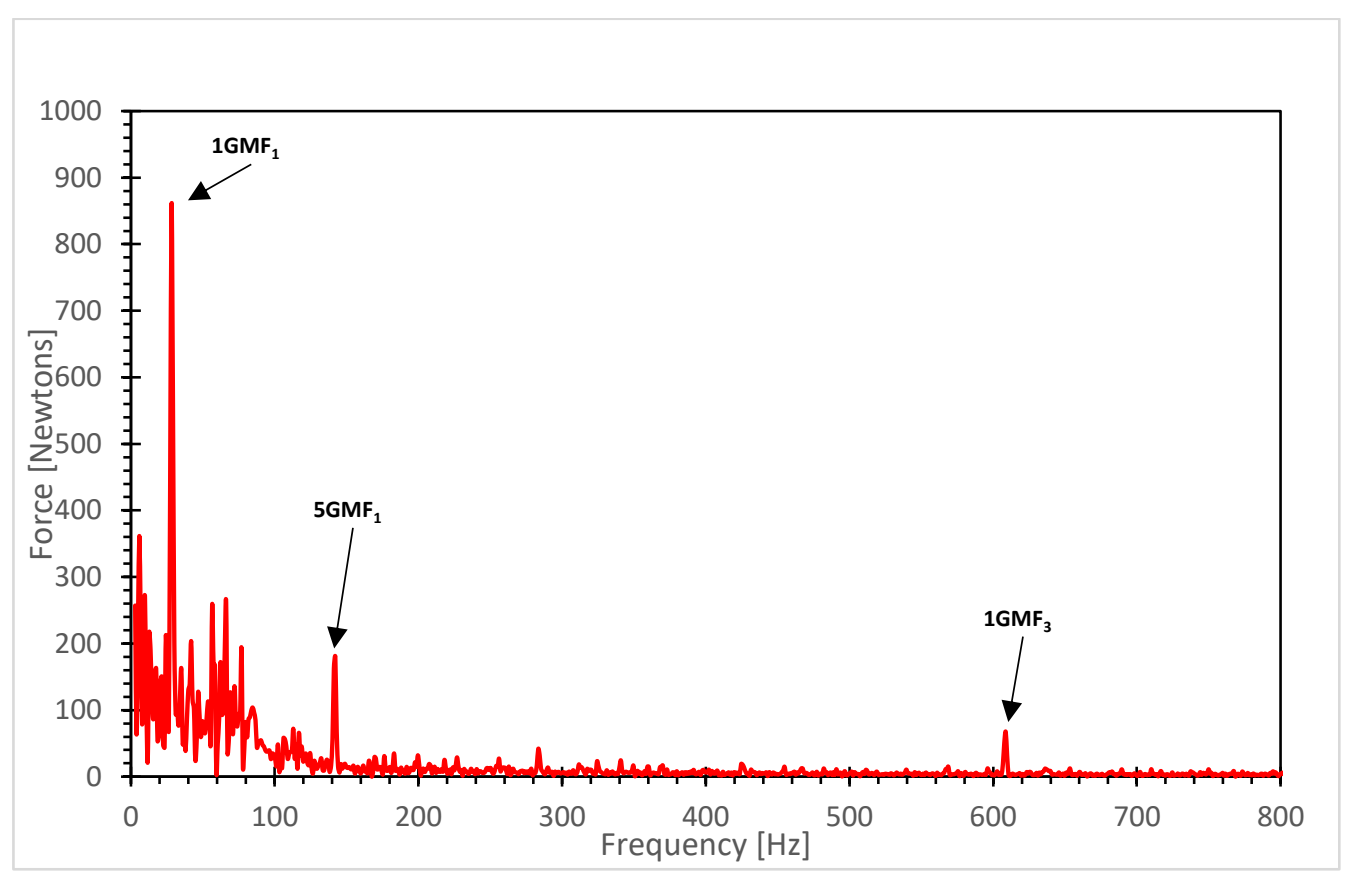

Figure 61. FFT of Stage 1 contact force between sun and planet gear in a full three stage assembly with a defect at first stage sun gear with input speed $1.5 \mathrm{rad} / \mathrm{sec}$. 


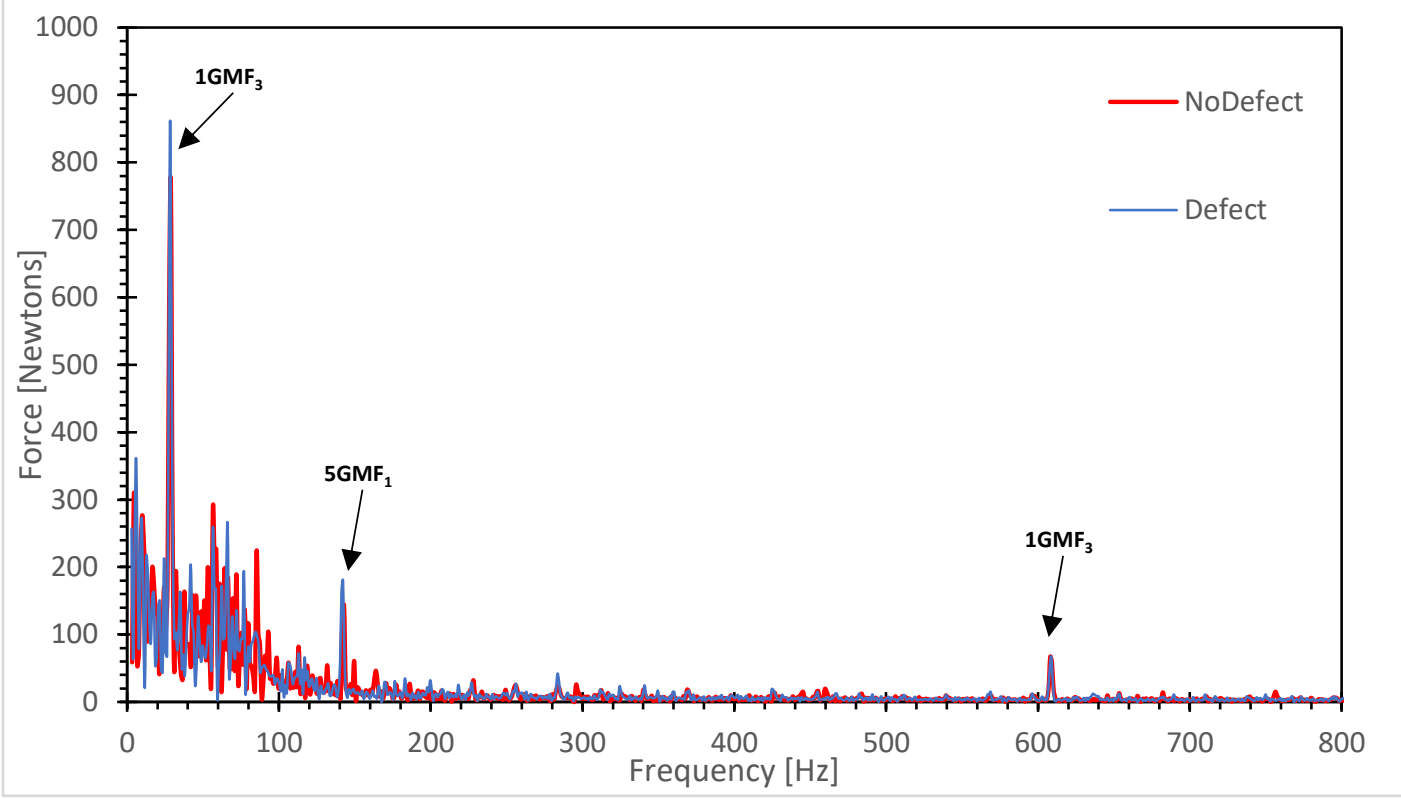

Figure 62. Stage 1 superposition comparison FFT of the contact force between the sun and planet gear with and without a defect at the sun gear tooth with input speed $1.5 \mathrm{rad} / \mathrm{sec}$.

Figures 60, 61, and 62 illustrate the first stage FFT of the system for both defective and nondefective gearboxes at 1.50rad/sec (14.3RPM) input operating speed. A similar behavior of the FFT is shown when compared previously to the system at $1.76 \mathrm{rad} / \mathrm{sec}$. At the first stage, three significant peaks can be seen. These occur at two harmonics of $\mathrm{GMF}_{1}, 5 \mathrm{GMF}_{1}$ and $1 \mathrm{GMF}_{1}$ as well as $1 \mathrm{GMF}_{3}$.

An FFT analysis was performed on the second stage of the assembly the same input speed, 1.50 $\mathrm{rad} / \mathrm{sec}$ The following figures, Figure 63, Figure 64 and Figure 65 show the FFT for at Stage 2 of the contact force between the sun and the planet gear with and without a defect at the first stage sun gear tooth for an input speed of $1.5 \mathrm{rad} / \mathrm{sec}$. 


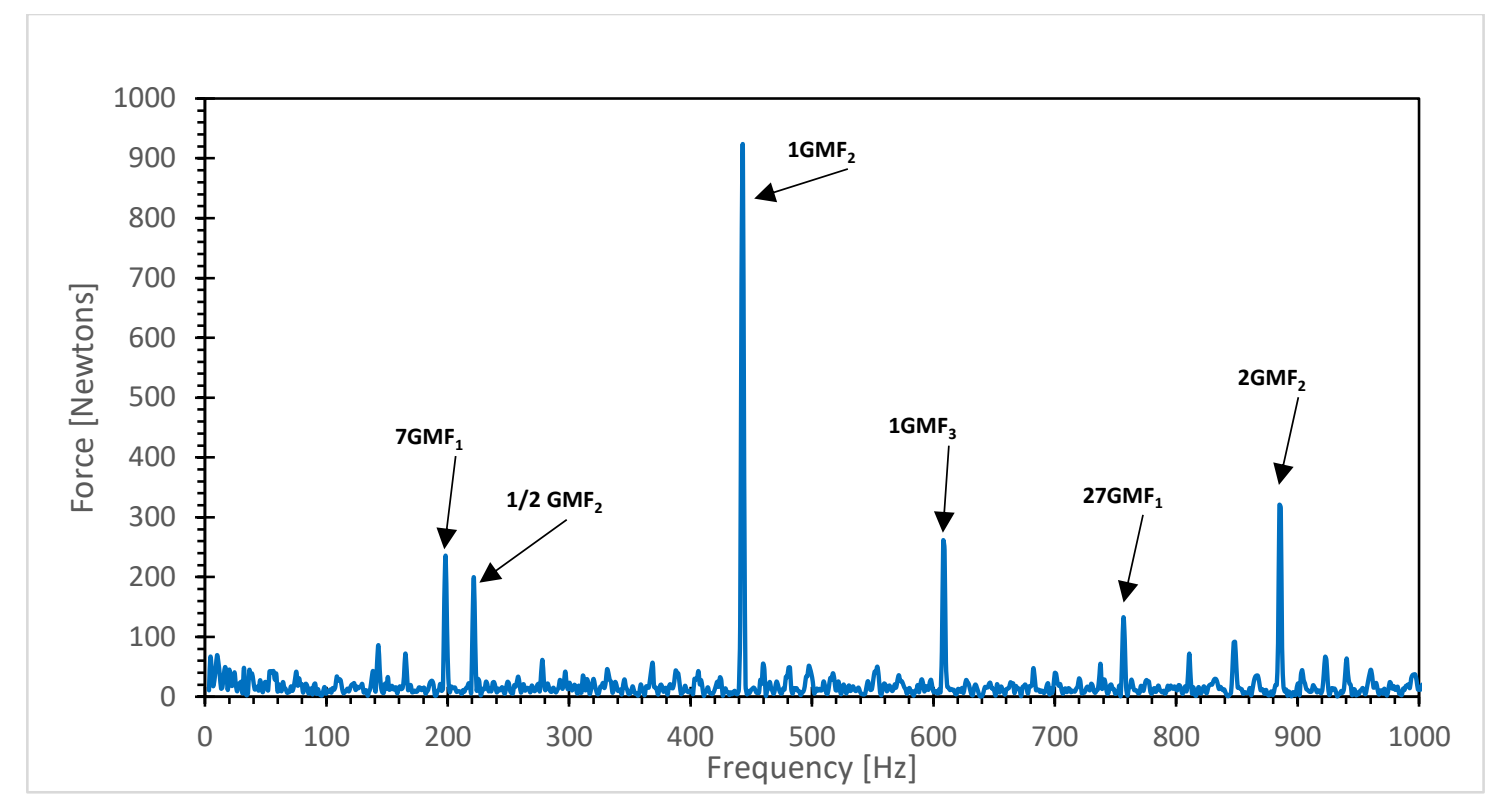

Figure 63. FFT of Stage 2 contact force between sun and planet gear in a full three stage assembly with input speed $1.5 \mathrm{rad} / \mathrm{sec}$.

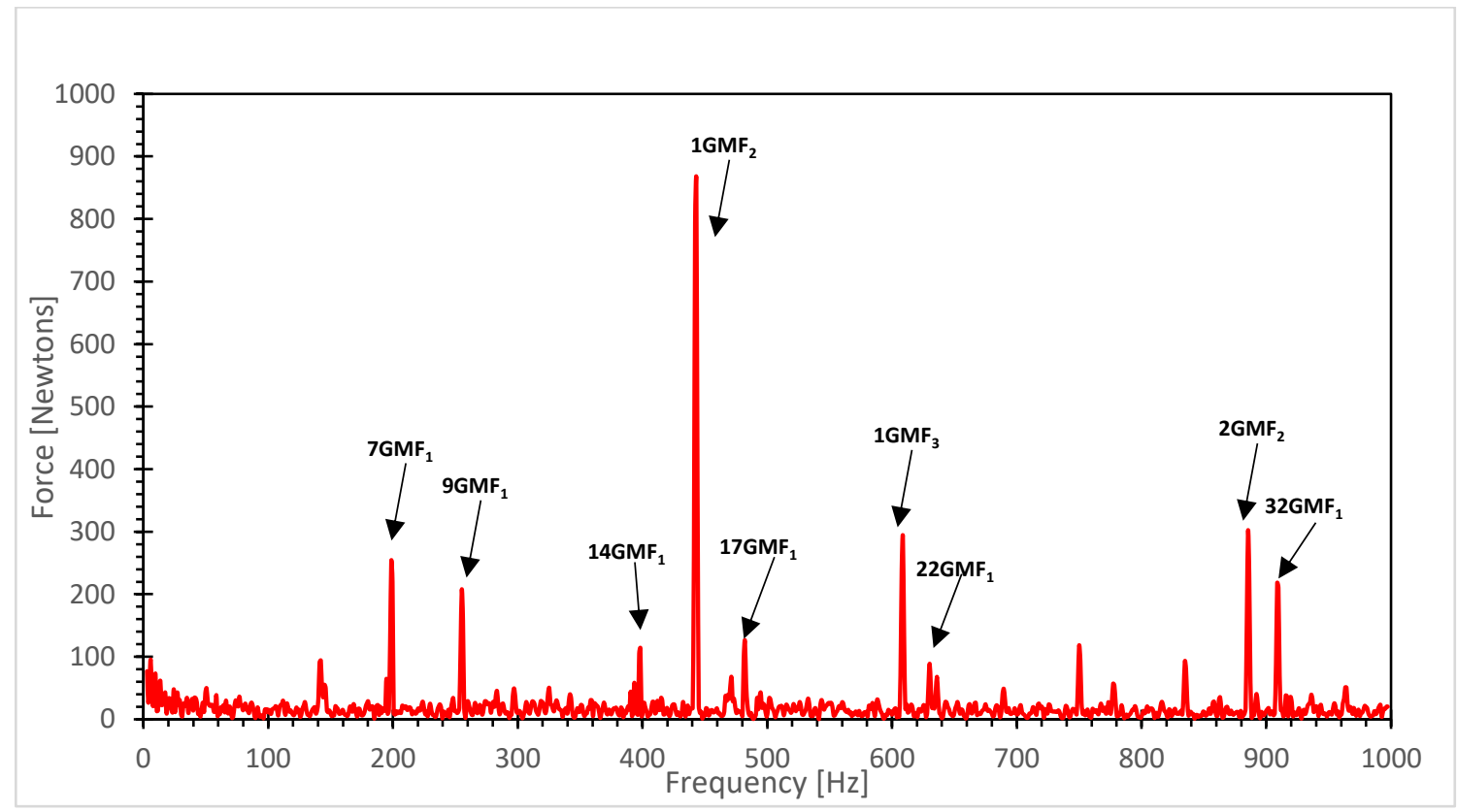

Figure 64. FFT of Stage 2 contact force between sun and planet gear in a full three stage assembly with a defect at first stage sun gear with input speed $1.5 \mathrm{rad} / \mathrm{sec}$. 


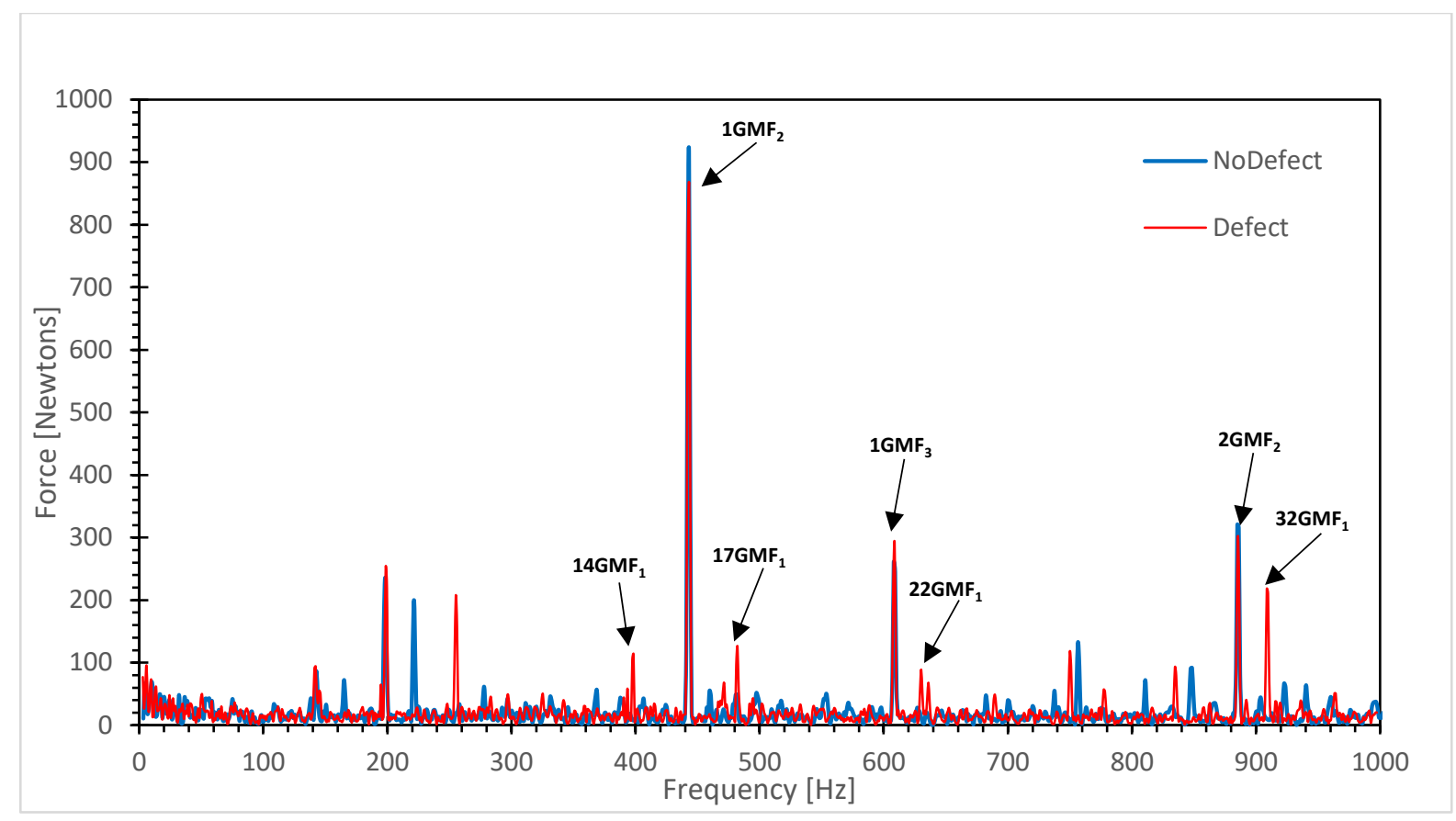

Figure 65. Stage 2 superposition comparison FFT of the contact force between the sun and planet gear with and without a defect at the sun gear tooth with input speed $1.5 \mathrm{rad} / \mathrm{sec}$.

Figures 63, 64, and 65 show the FFT at the second stage with and without a defect at the first stage sun gear. In Figure 65, the comparison between the two FFT's shows modulation of $1 \mathrm{GMF}_{2}, 2 \mathrm{GMF}_{2}$ and $1 \mathrm{GMF}_{3}$ by harmonics of $\mathrm{GMF}_{1}$. Two first stage harmonics modulate the dominate $1 \mathrm{GMF}_{2}$ and one $\mathrm{GMF}_{1}$ harmonic modulates $1 \mathrm{GMF}_{3}$ and $2 \mathrm{GMF}_{2}$. The similar occurrence to the system at $1.76 \mathrm{rad} / \mathrm{sec}$ is the modulation of $1 \mathrm{GMF}_{2}$ and $1 \mathrm{GMF}_{3}$. This can be determined as possible vibration signature to the defect.

The following figures, Figure 66, Figure 67 and Figure 68 show the FFT for at Stage 3 of the contact force between the sun and the planet gear with and without a defect at the first stage sun gear tooth for an input speed of $1.5 \mathrm{rad} / \mathrm{sec}$. 


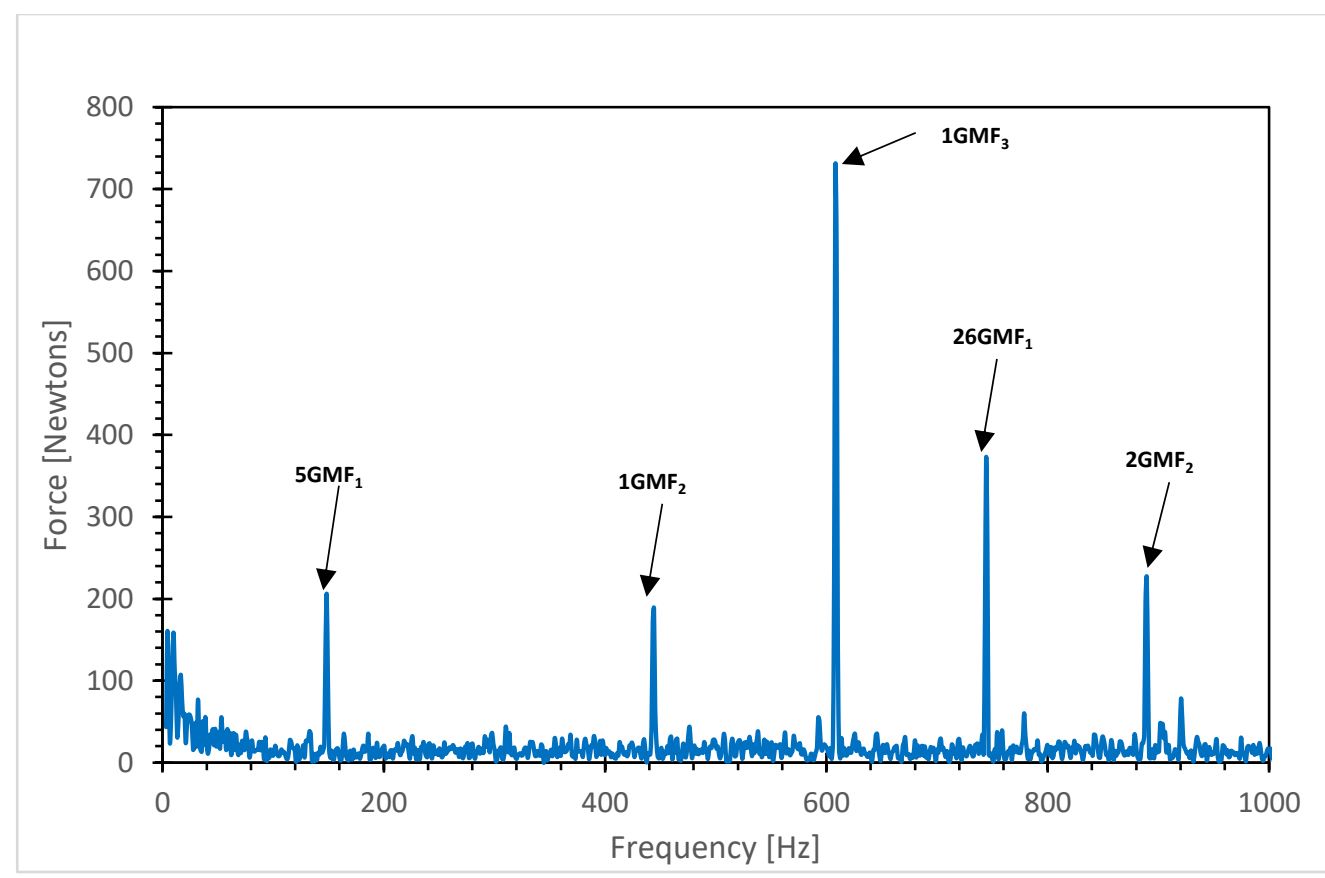

Figure 66. FFT of Stage 3 contact force between pinion and gear in a full three stage assembly with input speed $1.5 \mathrm{rad} / \mathrm{sec}$.

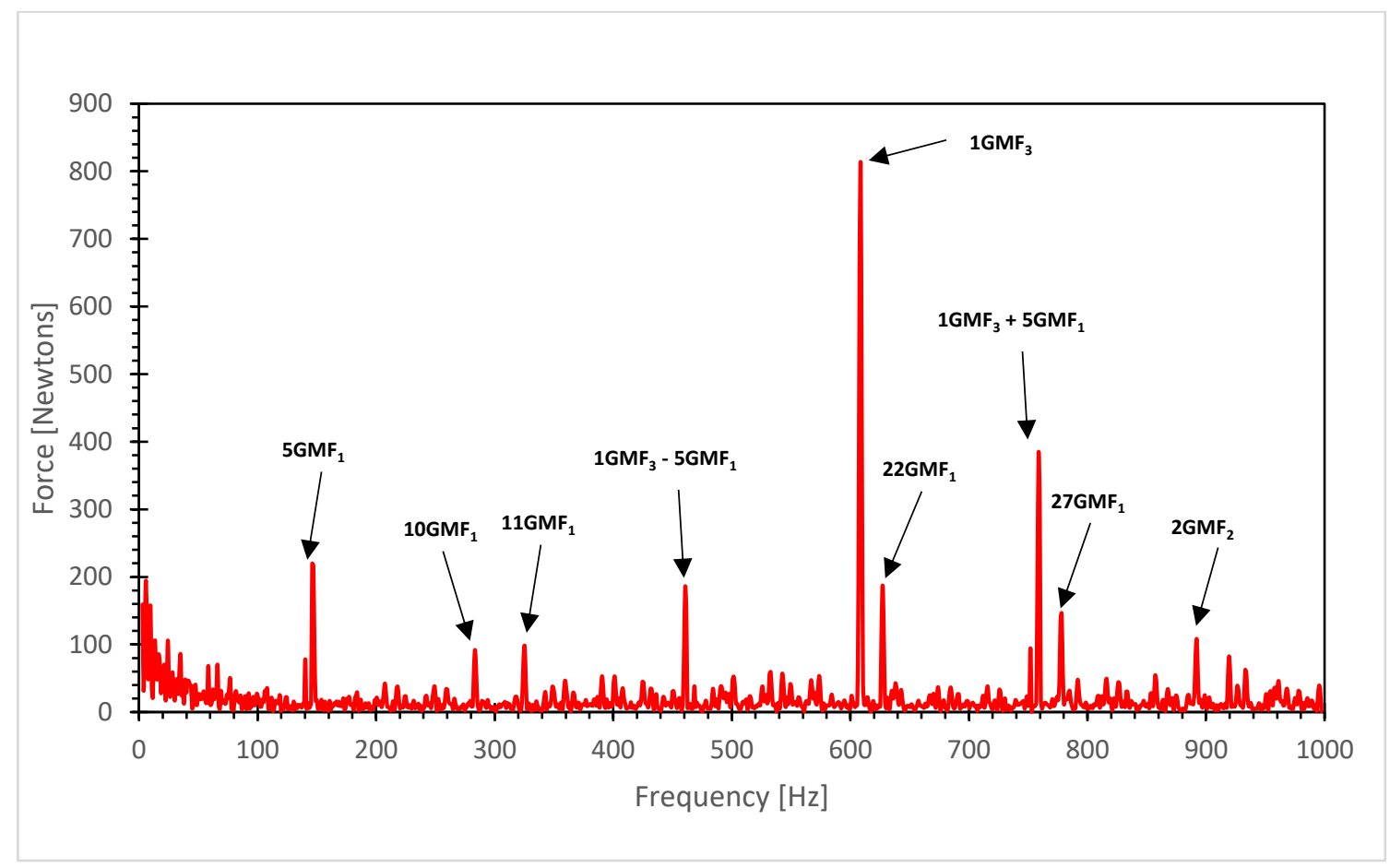

Figure 67. FFT of Stage 3 contact force between pinion and gear in a full three stage assembly with a defect at first stage sun gear with input speed $1.5 \mathrm{rad} / \mathrm{sec}$. 


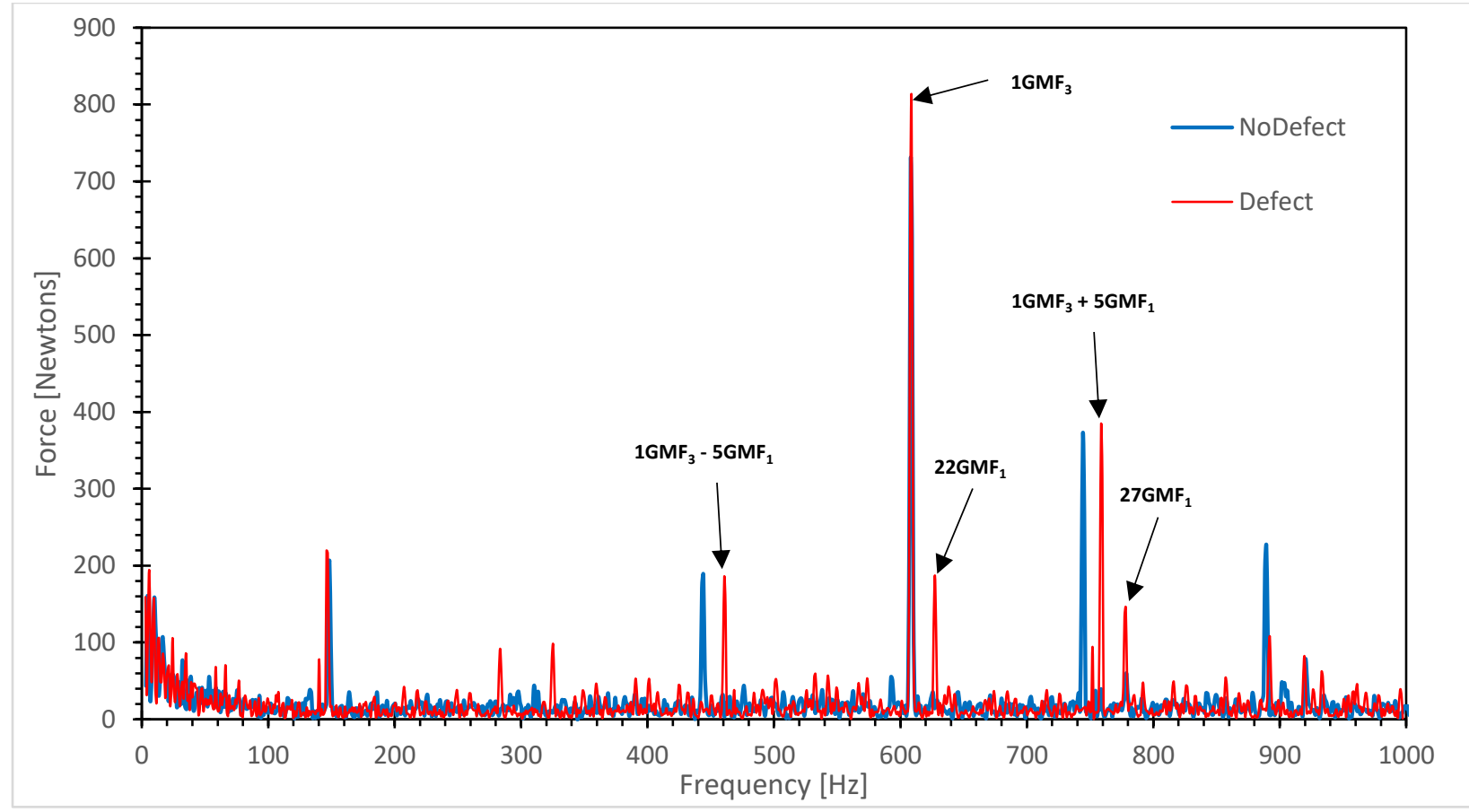

Figure 68. Stage 3 superposition comparison FFT of the contact force between the pinion and gear with and without a defect at the sun gear tooth with input speed $1.5 \mathrm{rad} / \mathrm{sec}$.

Figures 66, 67, and 68 show the FFT vibration pattern at the third stage with and without the defect at stage one sun gear. The superposition plot, Figure 68, highlights the significant peaks seen as the defect is imposed on the first stage sun gear. At the third stage FFT, it can be seen that sideband frequencies occur from the dominant $1 \mathrm{GMF}_{3}$ at both a lower and higher frequency. The sideband frequencies which are present are $1 \mathrm{GMF}_{3}-5 \mathrm{GMF}_{1}$ and $1 \mathrm{GMF}_{3}+5 \mathrm{GMF}_{1}$. Frequency modulation of the dominant $1 \mathrm{GMF}_{3}$ and the higher sideband, $1 \mathrm{GMF}_{3}+5 \mathrm{GMF}_{1}$, by harmonics of $\mathrm{GMF}_{1}$ is seen. It can also be noted that the sideband frequencies of $1 \mathrm{GMF}_{3}$ occurs at $1 \mathrm{GMF}_{3}$ plus/minus the second dominant frequency, $5 \mathrm{GMF}_{1}$, seen at the first stage FFT. This pattern is similar to the system at $1.76 \mathrm{rad} / \mathrm{sec}$.

The same simulation was performed on the system with a higher input speed at $2.0 \mathrm{rad} / \mathrm{sec}$. The FFT at the three stages were generated to define a vibration signature for this particular tooth defect at the first stage sun gear. The following figures, Figure 69, Figure 70 and Figure 71 show 
the FFT for at Stage 1 of the contact force between the sun and the planet gear with and without a defect at the first stage sun gear tooth for an input speed of $2.0 \mathrm{rad} / \mathrm{sec}(19.1 \mathrm{RPM})$.

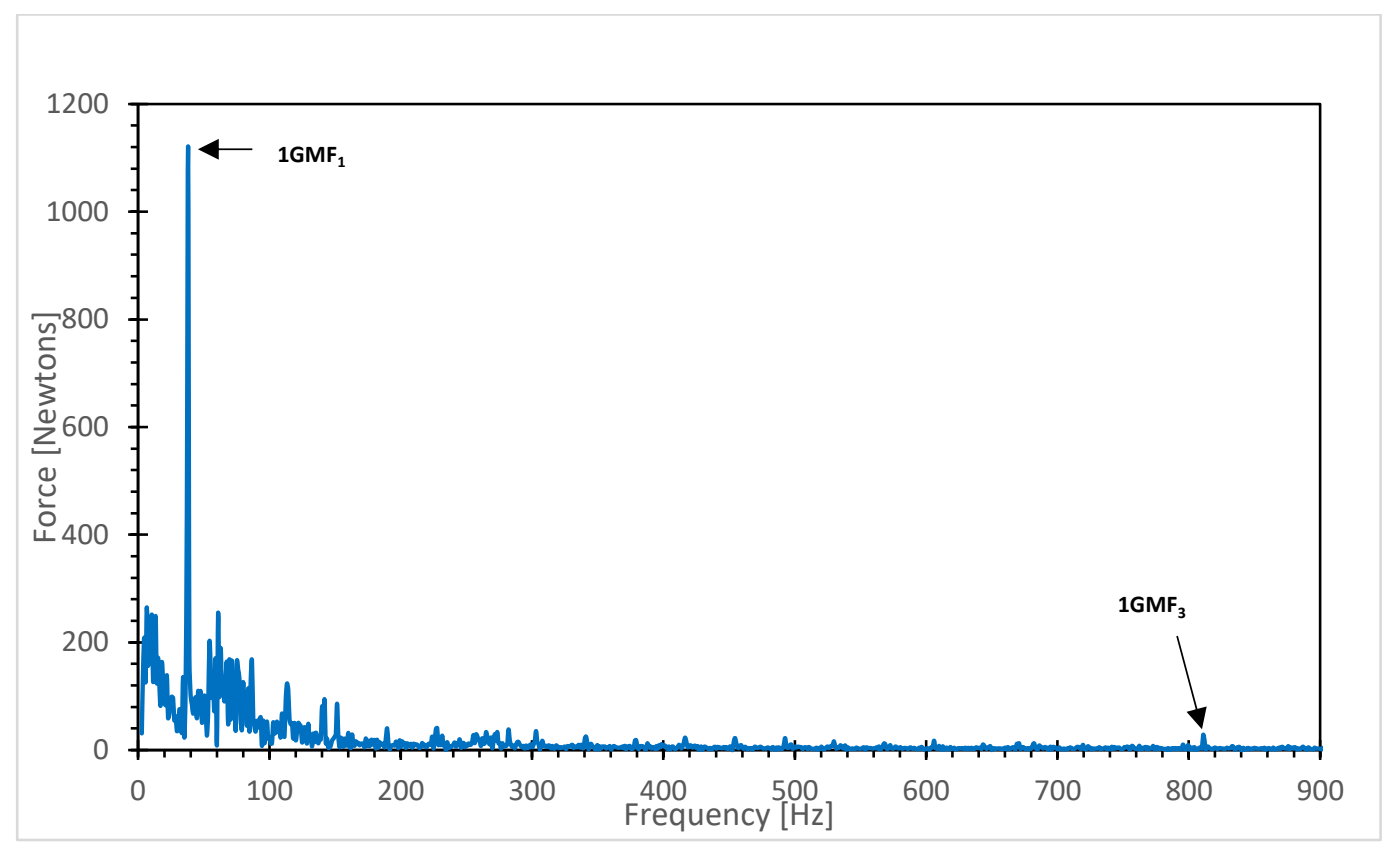

Figure 69. FFT of Stage 1 contact force between sun and planet gear in a full three stage assembly with input speed $2.0 \mathrm{rad} / \mathrm{sec}$. 


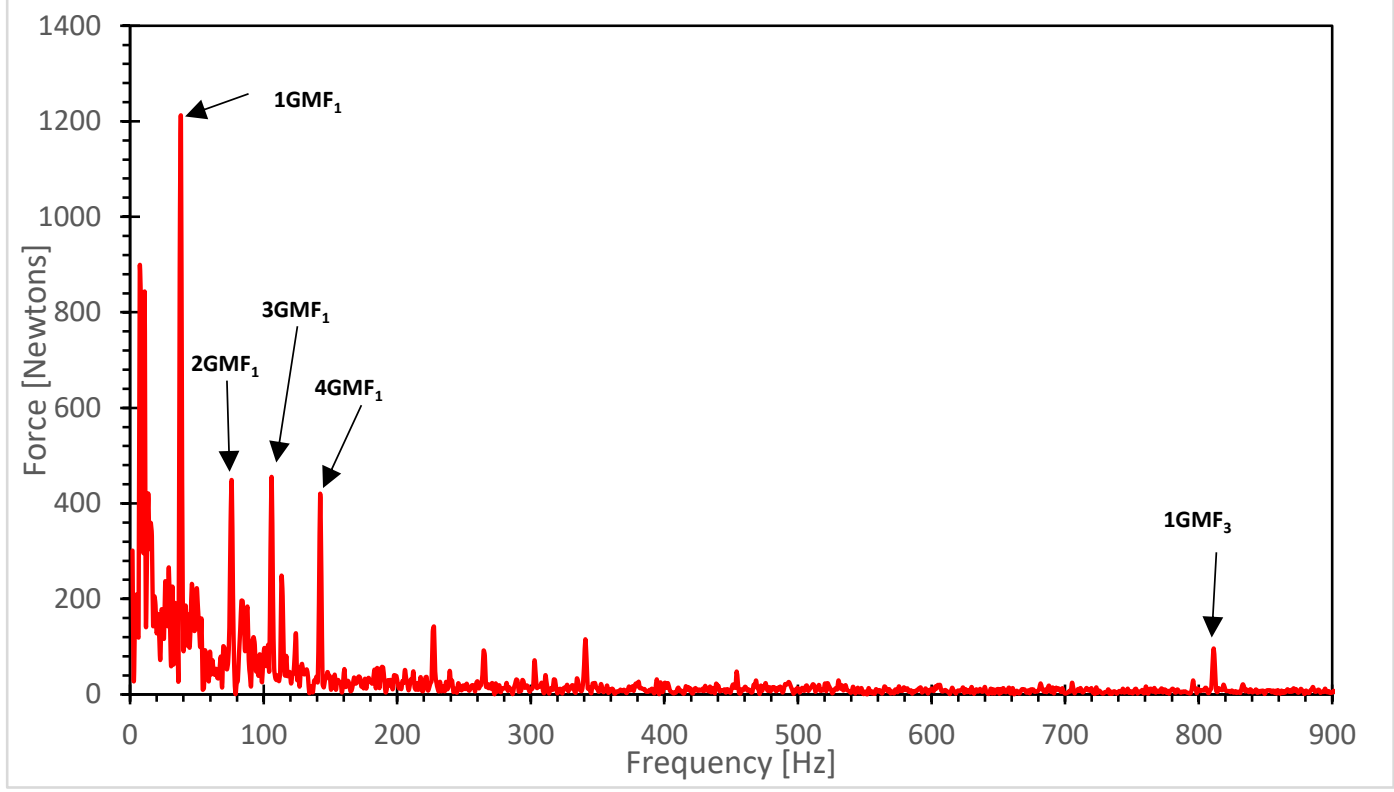

Figure 70. FFT of Stage 1 contact force between sun and planet gear in a full three stage assembly with a defect at first stage sun gear with input speed $2.0 \mathrm{rad} / \mathrm{sec}$.

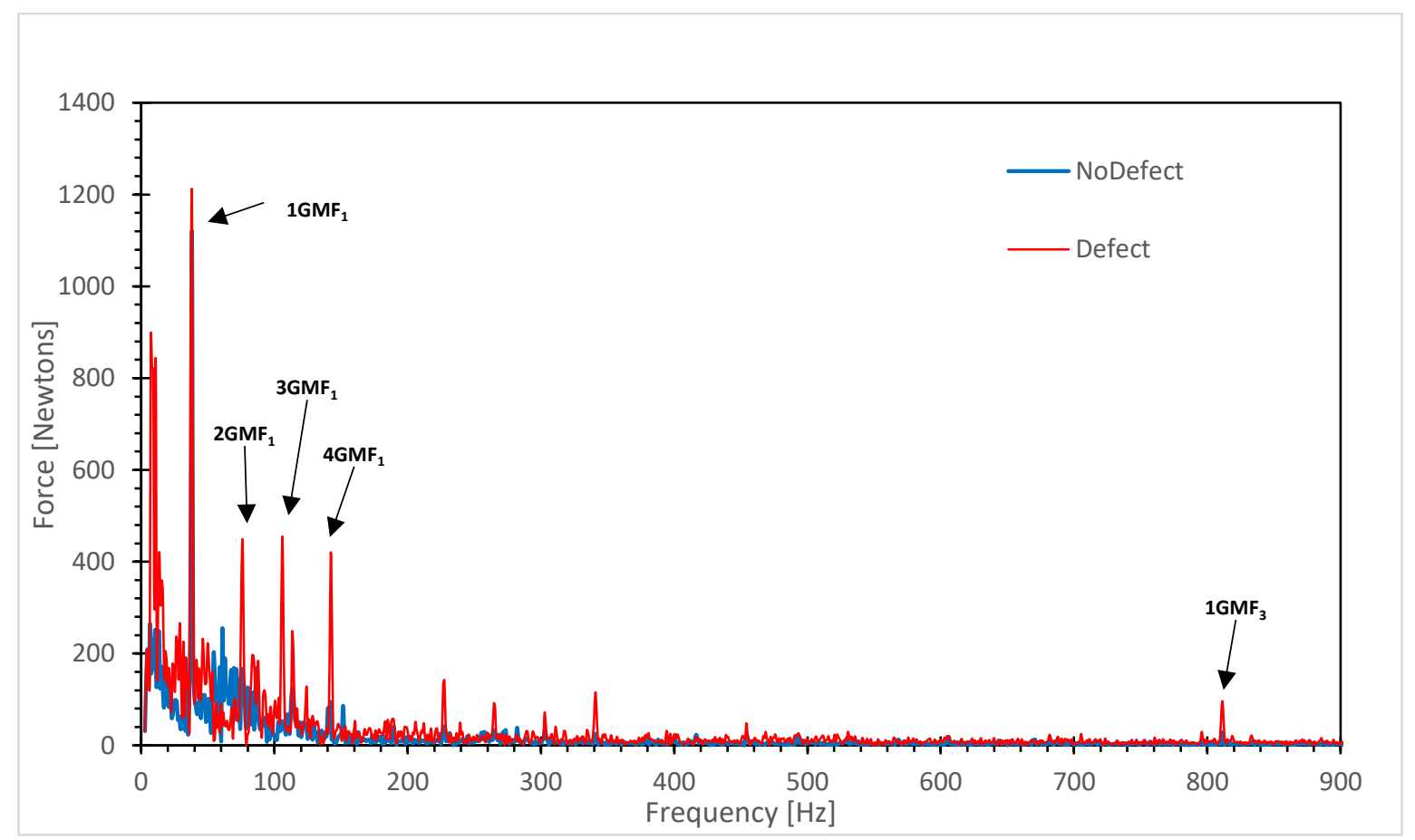

Figure 71. Stage 1 superposition comparison FFT of the contact force between the sun and planet gear with and without a defect at the sun gear tooth with input speed $2.0 \mathrm{rad} / \mathrm{sec}$. 
Figures 69, 70, and 71 shows the first stage FFT of the system with and without a defect at the first stage sun gear at $2.0 \mathrm{rad} / \mathrm{sec}(19.1 \mathrm{RPM})$ input operating speed. A similar behavior of the FFT is shown when compared previously to the system at $1.76 \mathrm{rad} / \mathrm{sec}$. At the first stage, two significant peaks can be seen on a non-defective gearbox at $1 \mathrm{GMF}_{1}$ and $1 \mathrm{GMF}_{3}$. On the defective gearbox more significant peaks occur at four harmonics of $1 \mathrm{GMF}_{1}, 2 \mathrm{GMF}_{1}, 3 \mathrm{GMF}_{1}$, $4 \mathrm{GMF}_{1}$ and $1 \mathrm{GMF}_{3}$ as well as other $\mathrm{GMF}_{1}$ harmonics at a much lower amplitude when compared to the first four of $\mathrm{GMF}_{1}$. The superposition plot, Figure 71, shows these significant peaks.

As with the other two operating speeds, the second stage FFT is generated. The following figures, Figure 72, Figure 73 and Figure 74 show the FFT for at Stage 2 of the contact force between the sun and the planet gear with and without a defect at the first stage sun gear tooth for an input speed of $2.0 \mathrm{rad} / \mathrm{sec}$. 


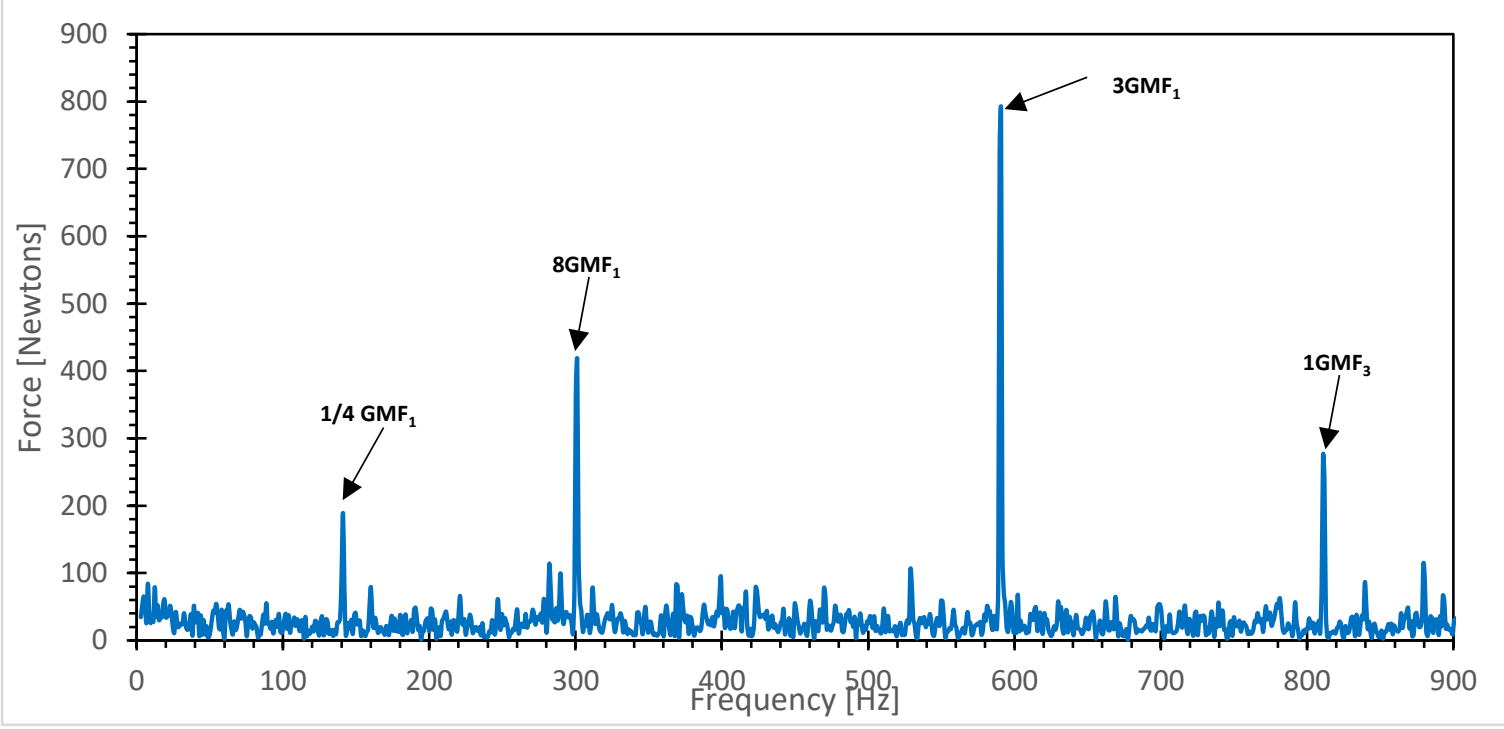

Figure 72. FFT of Stage 2 contact force between sun and planet gear in a full three stage assembly with input speed $2.0 \mathrm{rad} / \mathrm{sec}$.

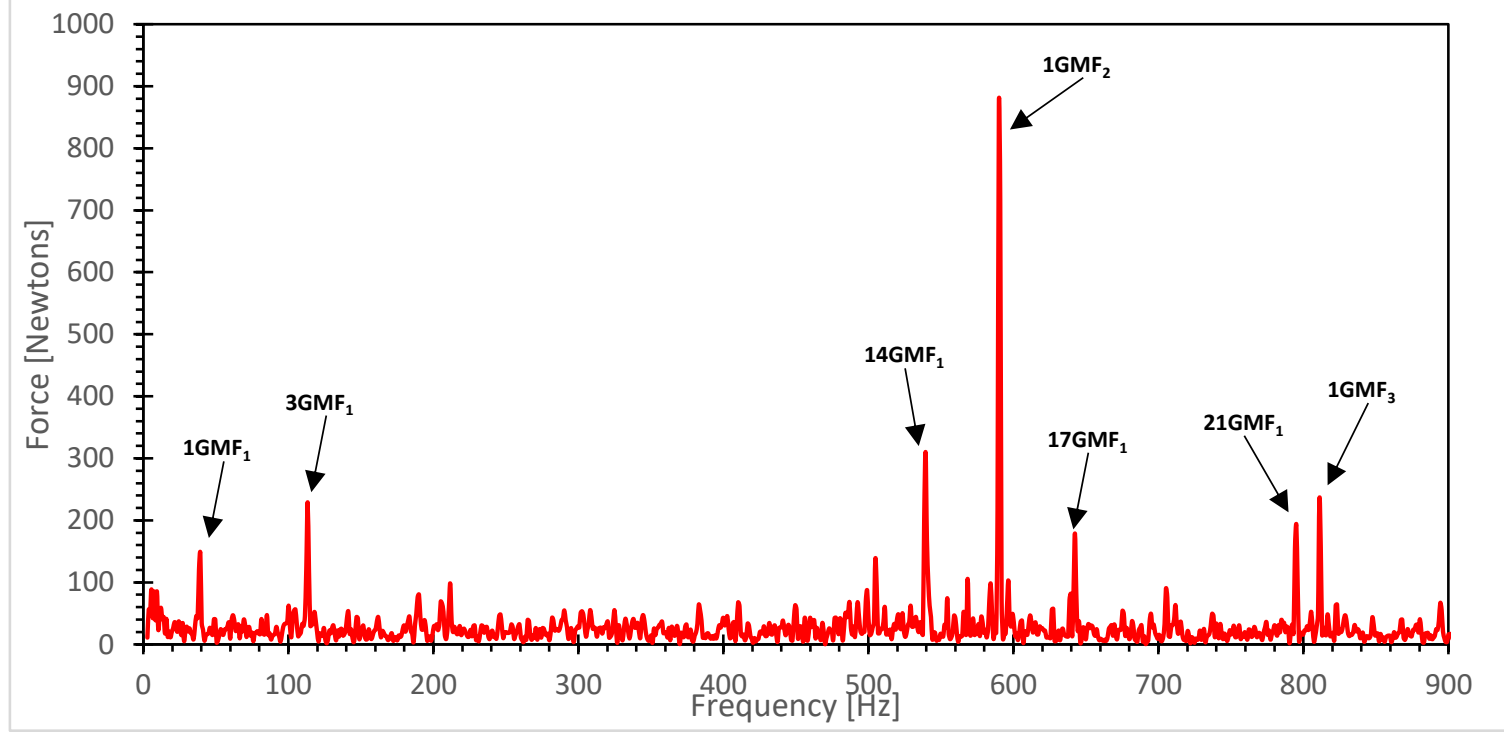

Figure 73. FFT of Stage 2 contact force between sun and planet gear in a full three stage assembly with a defect at first stage sun gear with input speed $2.0 \mathrm{rad} / \mathrm{sec}$. 


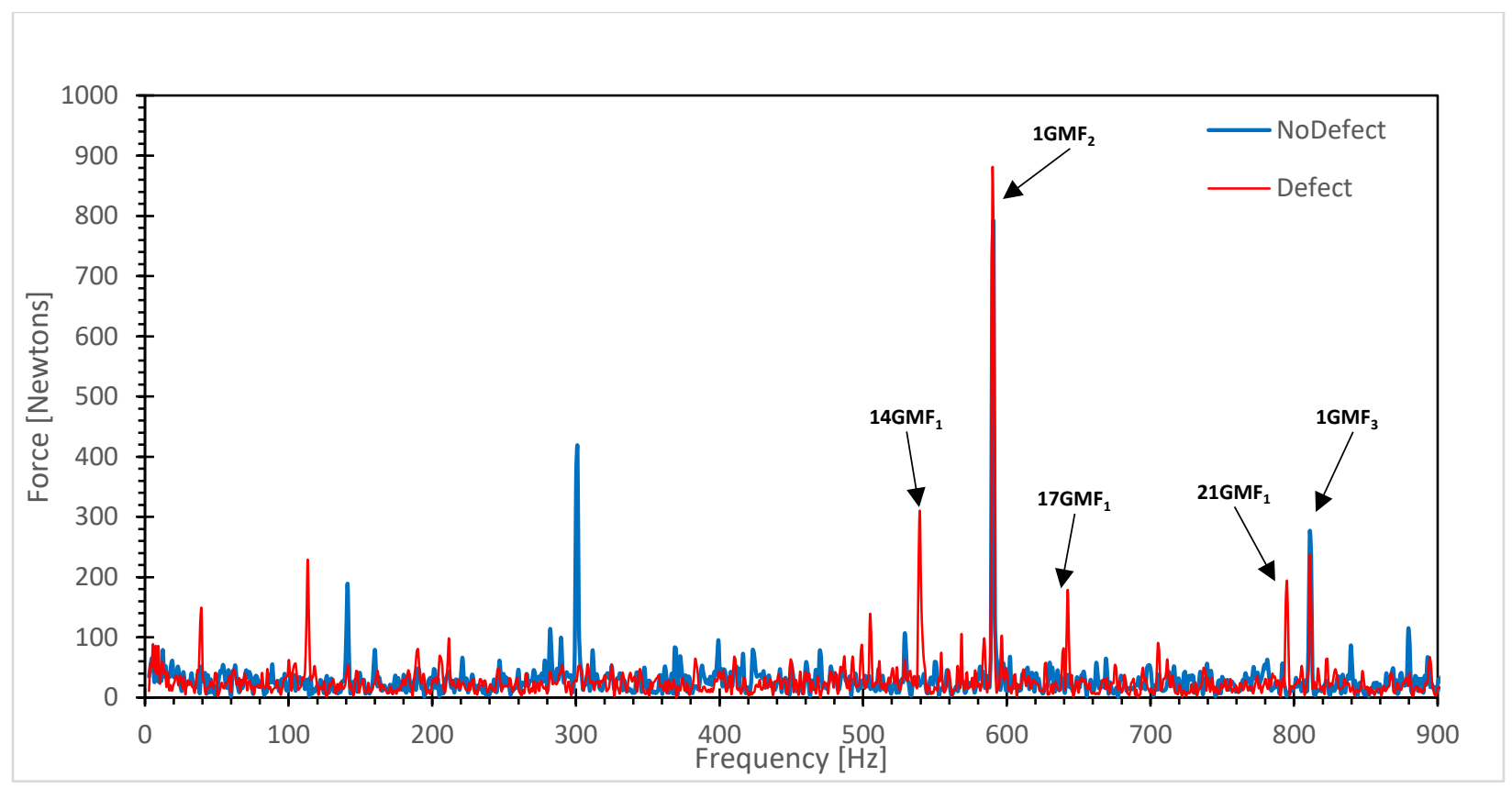

Figure 74. Stage 2 superposition comparison FFT of the contact force between the sun and planet gear with and without a defect at the sun gear tooth with input speed $2.0 \mathrm{rad} / \mathrm{sec}$.

Figures 72, 73, and 74 show the FFT at the second stage with and without a defect at the first stage sun gear. In Figure 74, the comparison between the two FFT's shows modulation of $1 \mathrm{GMF}_{2}$ and $1 \mathrm{GMF}_{3}$ by harmonics of $\mathrm{GMF}_{1}$. The dominant peak, $1 \mathrm{GMF}_{2}$ is modulated by two $\mathrm{GMF}_{1}$ harmonics, $14 \mathrm{GMF}_{1}$ and $17 \mathrm{GMF}_{1}$. The third stage $\mathrm{GMF}, 1 \mathrm{GMF}_{3}$, is modulated by $21 \mathrm{GMF}_{1}$. The similar occurrence to the system at $1.50 \mathrm{rad} / \mathrm{sec}$ and $1.76 \mathrm{rad} / \mathrm{sec}$ is the modulation of $1 \mathrm{GMF}_{2}$ and $1 \mathrm{GMF}_{3}$ by harmonics of $\mathrm{GMF}_{1}$. From the three different speed test, the modulation of $1 \mathrm{GMF}_{2}$ and $1 \mathrm{GMF}_{3}$ by harmonics of $\mathrm{GMF}_{1}$ can be determined as a vibration signature at the second stage of this three stage system with a defect on the tooth of the first stage sun gear.

As with the other system speed tests, this system is simulated at $2.0 \mathrm{rad} / \mathrm{sec}$ and the third stage FFT is developed. The following figures, Figure 75, Figure 76 and Figure 77 show the FFT for at 
Stage 3 of the contact force between the pinion and the gear with and without a defect at the first stage sun gear tooth for an input speed of $2.0 \mathrm{rad} / \mathrm{sec}$.

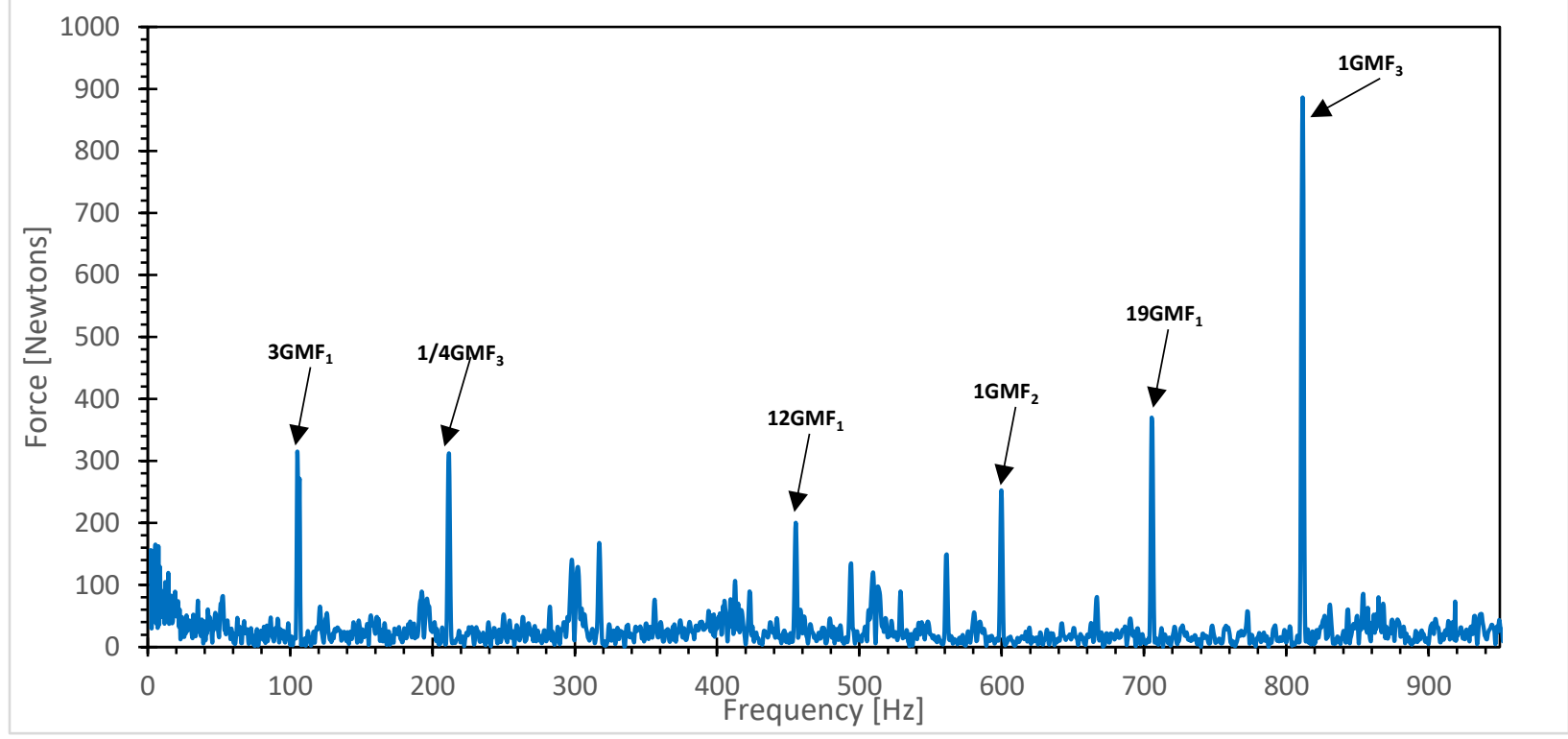

Figure 75. FFT of Stage 3 contact force between pinion and gear in a full three stage assembly with input speed $2.0 \mathrm{rad} / \mathrm{sec}$. 


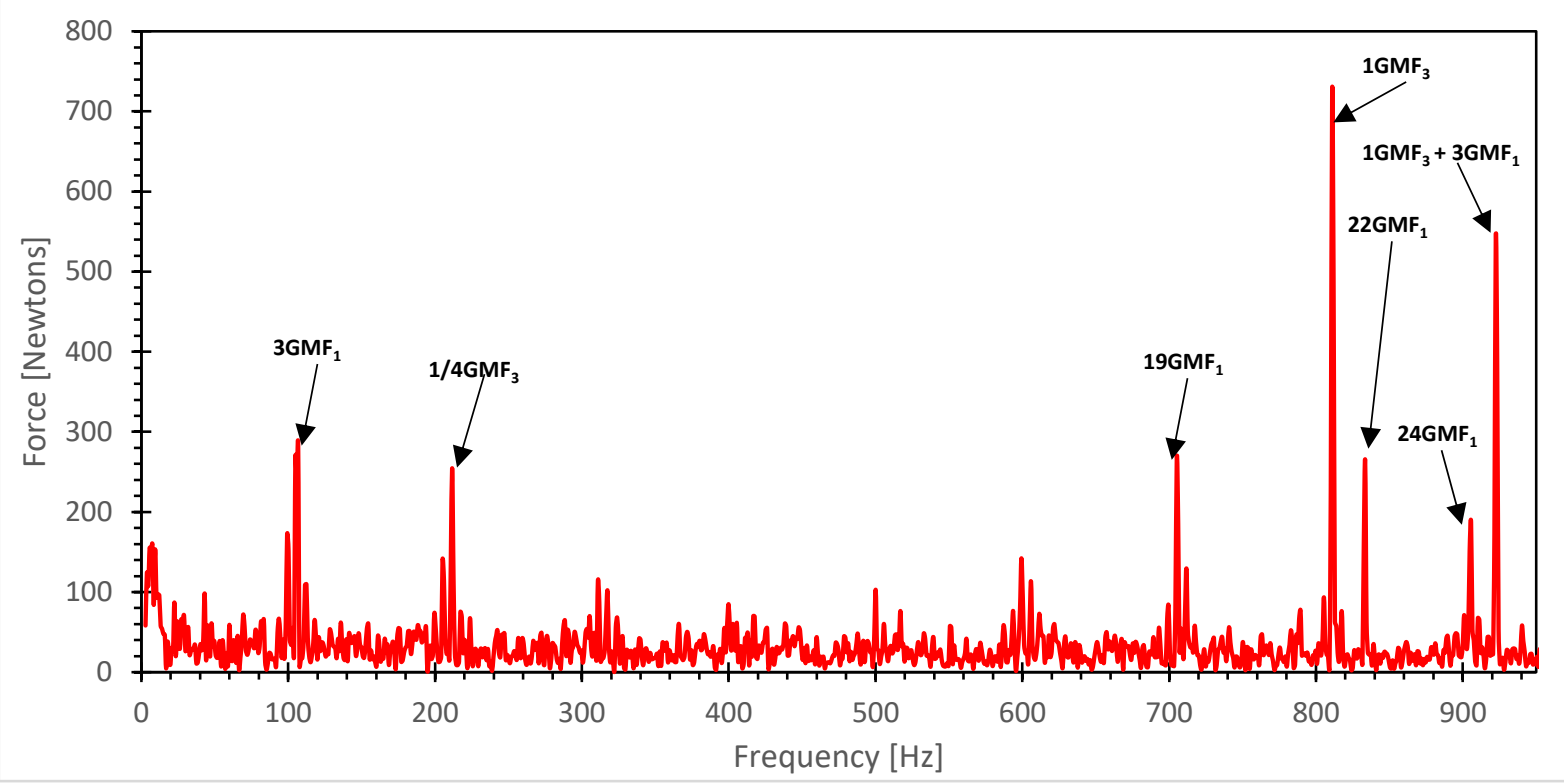

Figure 76. FFT of Stage 3 contact force between pinion and gear in a full three stage assembly with a defect at first stage sun gear with input speed $2.0 \mathrm{rad} / \mathrm{sec}$.

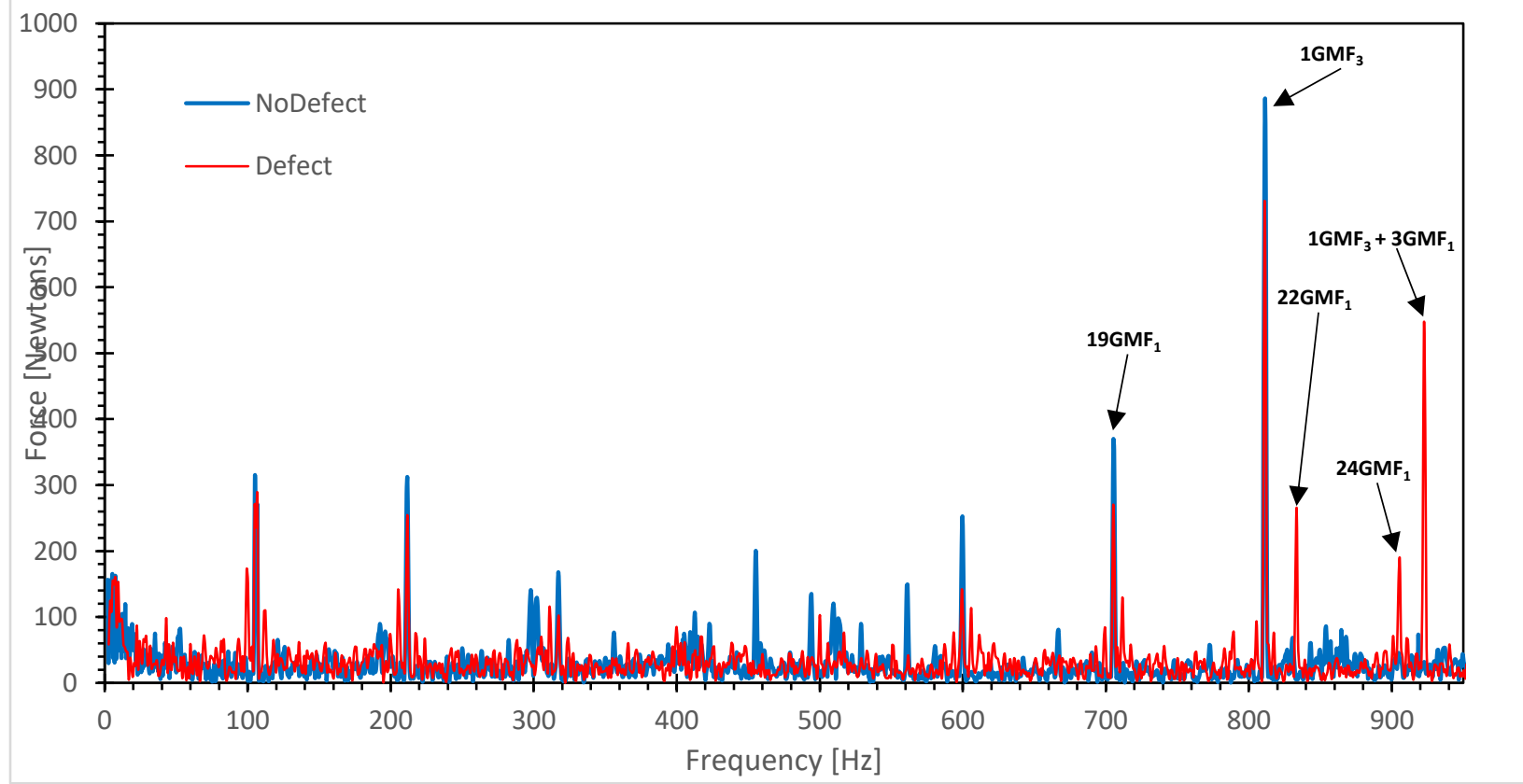

Figure 77. Stage 3 superposition comparison FFT of the contact force between the pinion and gear with and without a defect at the sun gear tooth with input speed $2.0 \mathrm{rad} / \mathrm{sec}$. 
Figures 75,76 , and 77 show the FFT vibration pattern at the third stage with and without the defect at stage one sun gear. The superposition plot, Figure 77, highlights the significant peaks seen as the defect is imposed on the first stage sun gear. A sideband frequency occurs from the dominant $1 \mathrm{GMF}_{3}$ at a higher frequency. The sideband frequency which is present is $1 \mathrm{GMF}_{3}+$ $3 \mathrm{GMF}_{1}$. Frequency modulation of the dominant $1 \mathrm{GMF}_{3}$ and the higher sideband, $1 \mathrm{GMF}_{3}+$ $3 \mathrm{GMF}_{1}$, by harmonics of $\mathrm{GMF}_{1}$ can be seen to occur. The sideband frequency present in this FFT, occurs at $1 \mathrm{GMF}_{3}+3 \mathrm{GMF}_{1}, 3 \mathrm{GMF}_{1}$ is one of the significant frequencies which occurs at when looking the first stage FFT of the system. This pattern is similar to the system at both 1.50 $\mathrm{rad} / \mathrm{sec}$ and $1.76 \mathrm{rad} / \mathrm{sec}$. This pattern can be determined as vibration signature seen at the third stage FFT of a full system assembly due to a defect at the first stage sun gear. 


\section{CHAPTER 7. CONCLUSION}

The vibration signature for a three stage wind turbine gearbox representing the Nordex N90 with a defect was developed. With the methods outlined in this these for incorporating a flexible body through the use of MSC Patran/Nastran into Adams. The stress at a desired location can be acquired under the dynamic operations of the system which is more realistic as to the more classical static stress analysis or impact analysis in the classical FEA software. The use MSC Patran/Nastran allows for the ease of integration between the finite element model into a dynamic system. The method of development for the vibration characteristics of this system developed in this thesis can be used to further study and simulated a variety of cases and defects which can occur in a wind turbine gearbox.

The strength MSC Adams is highlighted by the ability to simulate dynamic interaction between bodies and generate vibration results and stress results due to the integration of a flexible body. This allows for the first steps in a development of a health monitoring method for a gearbox. In this case, the targeted gearbox is the Nordex N90 wind turbine. With the availability of the working hardware, a significant limitation is the computational time required for simulations. Model scale reduction was used for computational efficiency purposes in the simulations.

Limitations to stress analysis to defects such as the one investigated in this thesis is the method in which the flexible body is generated for integration into MSC Adams from MSC Patran. The Craig-Bampton modal analysis used on the body is a linear superposition of modes. This method only captures the linear behavior of the system and cannot be used with non-linear analysis methods, as confirmed when discussing with a Patran expert at MSC. This means that non-linear geometry or material behavior cannot be captured through this method of dynamic stress

analysis. In terms of defects at the gear teeth root, the defect model represented in this research is 
a notch model created at the root of the tooth. This can be representative of a crack which has occurred at the root. MSC Patran has the capability to perform crack propagation methods which would generally be ideal for such an analysis. However, crack propagation through ungluing elements, as it is referred to in Patran, is a non-linear method and is not compatible with the Craig-Bampton method.

With the methods developed in this thesis, it is hopeful that further research can be conducted into gearbox health monitoring with a variety of different types of defects and integration of different elements seen in a typical system. The model for the N90 gearbox is developed based on previous research on this wind turbine as with limited information provided by Nordex themselves, in the hopes of creating a model which can be representative of the true N90 system. However, without a schematic provided by Nordex, it would be impossible to create a perfect representation.

There are certain aspects with both MSC Adams and MSC Patran that may defer other uses from utilizing the full strength of the software. The most challenging aspect to navigate around is the user interface (UI) of both software. A tutorial on gear flexible body development is provided with this document with the hopes that it will significantly reduce the time to develop a working system model for further research. Certain aspects of the Adams software are inefficient and confusing such as developing a working model. With different changes and improvements to the UI, different processes used in this research can be performed with ease. Both MSC Adams and Patran are very powerful software especially with multibody dynamic analyses however, a lot of its capabilities are hidden behind what maybe classified as an outdated user interface when compared to those of ANSYS, Abaqus, Solidworks, or Creo Direct. 


\section{REFERENCES}

[1] "Nordex N90 Specification Sheet" Nordex SE

[2] Sheng S. "Wind Turbine Gearbox Condition Monitoring Round Robin Study - Vibration Analysis”, National Renewable Energy Laboratory, 2012.

[3] "Wind Turbine Gear Failure Modes - A Brief”, National Renewable Energy Laboratory ASME/STLE 2011 International.

[4] Puigcorbe J., and De-Beaumont A., 2010, "WindTurbine Gearbox Reliability: The impact of rotor support,” Renewable Energy World Magazine.

[5] Norton, Robert L. Machine Design an Integrated Approach. Prentice Hall, 2014.

[6] D. W. Aitken, “Transitioning to A Renewable Energy Future”, ISES White Paper, 2003.

[7] Ribrant J., and Bertling L. M., 2007, "Survey ofFailures in Wind Power Systems With Focus on Swedish Wind Power Plants During 1997-2005,” IEEE Transactions on Energy Conversion.

[8] Alemayehu, Fisseha M. and Ekwaro-Osire, Stephen "Loading And Design Parameter Uncertainty In The Dynamics And Performance Of High-Speed-Parallel-Helical Stage Of A Wind Turbine Gearbox"

[9] Özgüven and Houser, "Mathematical Models used in Gear Dynamics - A Review," Journal of Sound and Vibration, no. 121(3).

[10] Smolders K., Long H., Feng Y., and Tavner P., 2010,"Reliability Analysis and Prediction of Wind Turbine Gearboxes,” European Wind Energy Conference,Warsaw, Poland. 
[11] Sawatzky, Rene P. D. "Vibration Based Planetary Gear Analysis and Damage Detection"

[12] A. Milburn, "Wind Turbine Gearbox Wear and Failure Modes and Detection Methods," 2011.

[13] Musial W., Butterfield S., and McNiff B., 2007,“Improving wind turbine gearbox reliability," European Wind Energy Conference, Milan, Italy.

[14] Kong, Meagher, Xu, Wu and Wu, "Nonlinear Contact Analysis of Gear Teeth for Malfunction Diagnostics," IMAC XXVI a Conference on Structural Dynamics, Society for Experimental Mechanics, 2008.

[15] J. Giesbers, "CONTACT MECHANICS IN MSC ADAMS," University of Twenty, 2012.

[16] Wu, Meagher and Sommer, "A Differential Planetary Gear Model with Backlash and Teeth Damage," IMAC XXIX a Conference and Exposition on Structural Dynamics, Society for Experimental Mechanics, 2011.

[17] Bradaric, G. "Dynamic analysis of connecting rods usinc msc adams modal synthesis method for flexible bodies" 2017

[18] Sommer, Meagher and Wu, "Gear Defect Modeling of a Multiple-Stage Gear Train," Modellingand Simulation in Engineering, 2011.

[19] Lin and Parker, "Analytical Characterization of the Unique Properties of Planetary Gear Free Vibration," ASME Journal of Vibration and Acoustics, 1999.

[20] K. L. Johnson, Contact mechanics, Cambridge: Press Syndicate of the University of Cambridge, 2004. 
[21] Verheul, Chris “ADAMS Methodology: Contact Modeling” MSC Software, SayField International, Lecture.

[22] Spencer, A. J. M. Continuum mechanics. Dover Publications, 2014.

[23] Audio, NTi. "How an FFT works". www.nti-audio.com.

[24] Craig, Roy, and Mervyn Bampton. "Coupling of substructures for dynamic analyses." AIAA journal 6.7 (1968): 1313-1319.

[25] Alshabatat, Nabeel \& Naghshineh, Koorosh. (2014). Optimization of Natural Frequencies and Sound Power of Beams Using Functionally Graded Material. Advances in Acoustics and Vibration. 2014. 1-10. 10.1155/2014/752361.

[26] Fundamentals of Structural Dynamics Roy R. Craig... (517 Textbook)

[27] MSC Software Corporation. "Adams Flex Help (for Adams 2017.1).” 23 Mar. 2017.

[28] Ottarson, Gisli, Greg Moore, and Diego Minen. "MDI/ADAMS-MSC/NASTRAN Integration Using Component Mode Synthesis." (1998).

[29] Budynas Richard G, Nisbett Keith J. "Shigley's Mechanical Engineering Design.”, McGraw-Hill Education, 1 Jan. 1970. 


\title{
APPENDICES
}

APPENDIX A - PATRAN TUTORIAL

\section{MSC Patran to Adams Flexible Body Integration for an Output Gear in a Planetary Gear System Assembly: A Tutorial}

\author{
Tananant (Mel) Boonya-ananta \\ tboonyaa@calpoly.edu \\ Mechanical Engineering Department \\ California Polytechnic State University \\ San Luis Obispo, CA 93407
}

Disclaimer: This tutorial assumes basic knowledge of Solidwork or other CAD software and Adams View. This document will walk through the process of creating a flexible body in MSC Patran and a quick note on system integration into MSC Adams.

This tutorial is developed from the Youtube video referenced bellow which creates a similar analysis using a crank/slider system.

"Flexible Body Integration Using Adams and MSC Nastran"

https://www.youtube.com/watch?v=dd9rjSe27rU 


\section{Tutorial}

Prepare a model in Solidworks or other CAD programs and save as a Parasolid file. For the purposes of this tutorial a model has been provided in the same directory named "Stage1NoCrack.x_t". Note that .x_t and .x_b file types are both Parasolid files and will work for this tutorial.

The part is prepartitioned in Soldiworks using the split face tool to generate independent surfaces for meshing. This is show below.

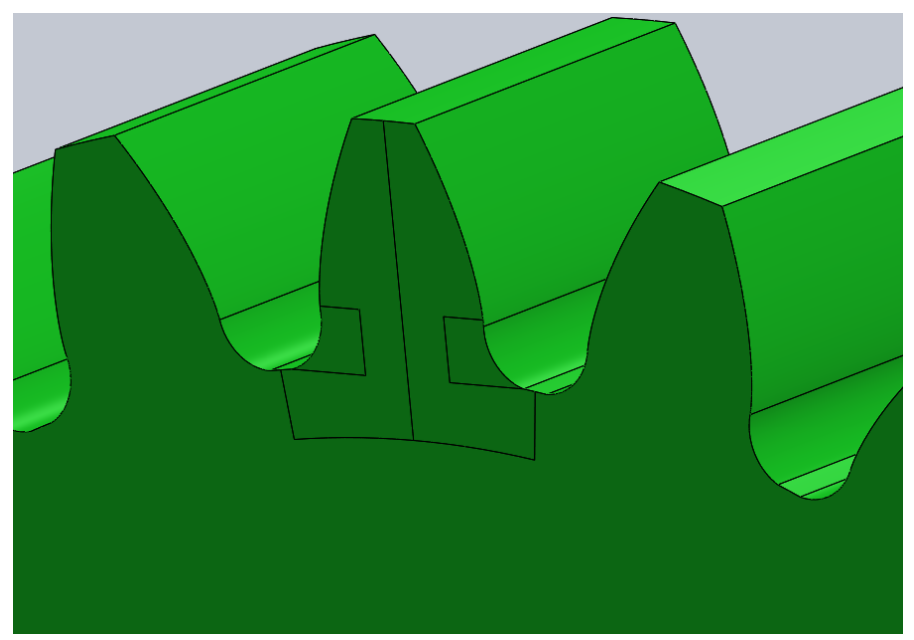

Start by opening MSC Patran. The icon will look similar, if not exactly the same as this.

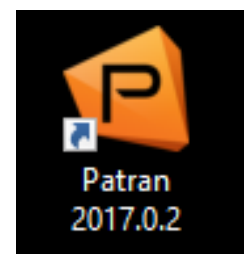

Before creating the database for the analysis, it is recommended that the Parasolid file is in the same working directory (preferably a newly created one), this will ensure that all related files are created and remain in the same location. This Parasolid file will also be the same file that will be used to create the model in Adams.

Once Patran is open, navigate to the Home tab and click the "New Database" icon. Name and create the new database in the working directory. Leave default templates. Click "OK". 

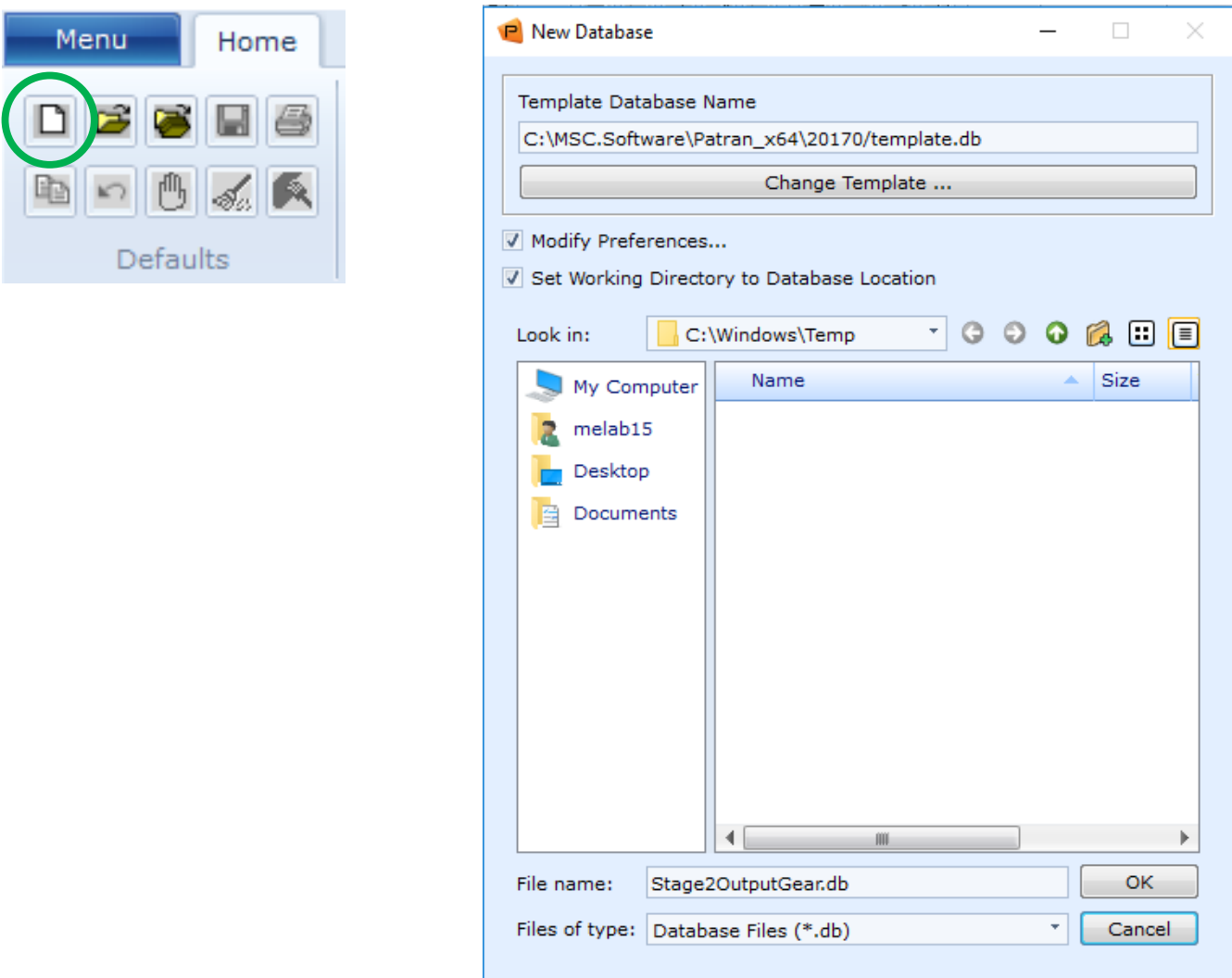
A right hand side (RHS) window will pop up, accept default settings click "OK".

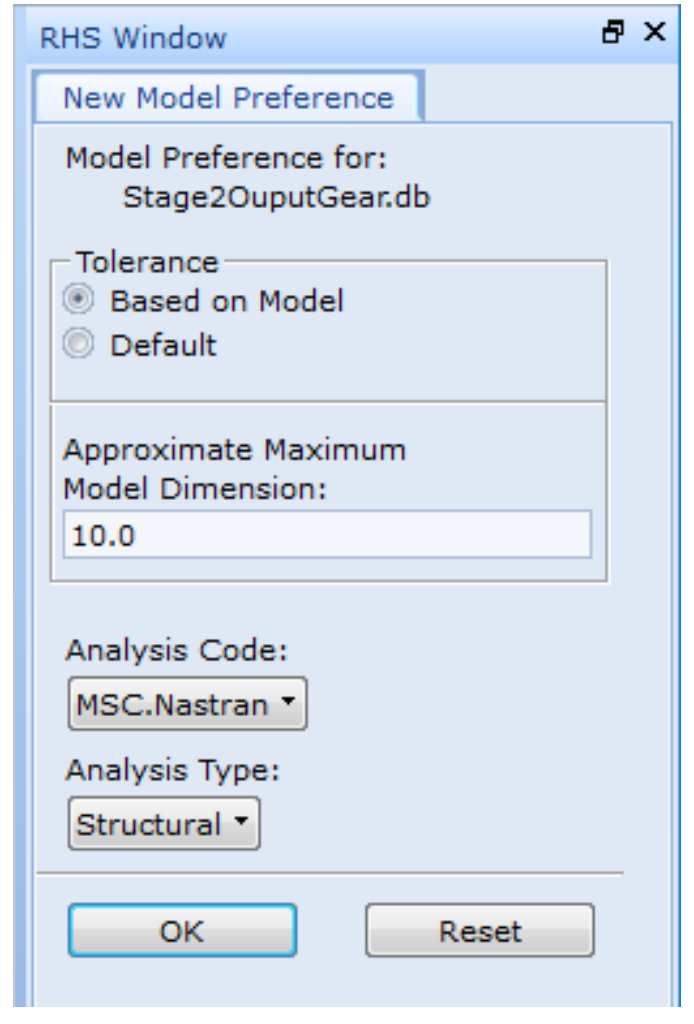

Navigate

appear. Mouse over "File" and click "Import".

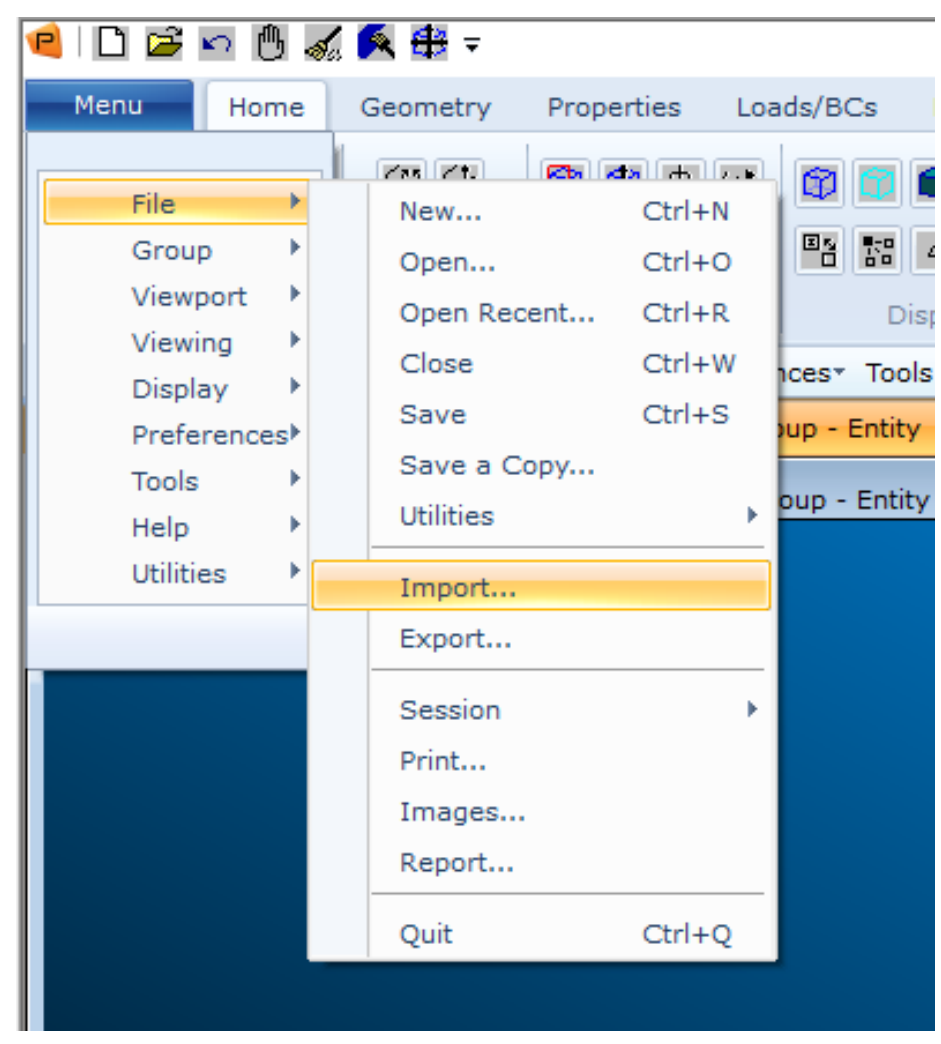


A new window will pop up. Select "Stage1NoCrack.x_t". This model is created in Metric units under MMKS unit convention. This indicates that the main unit of length is MILLIMETERS. On the right side of the import window, click "Parasolid xmt Options...". A RHS menu will open select "Model

Units..."
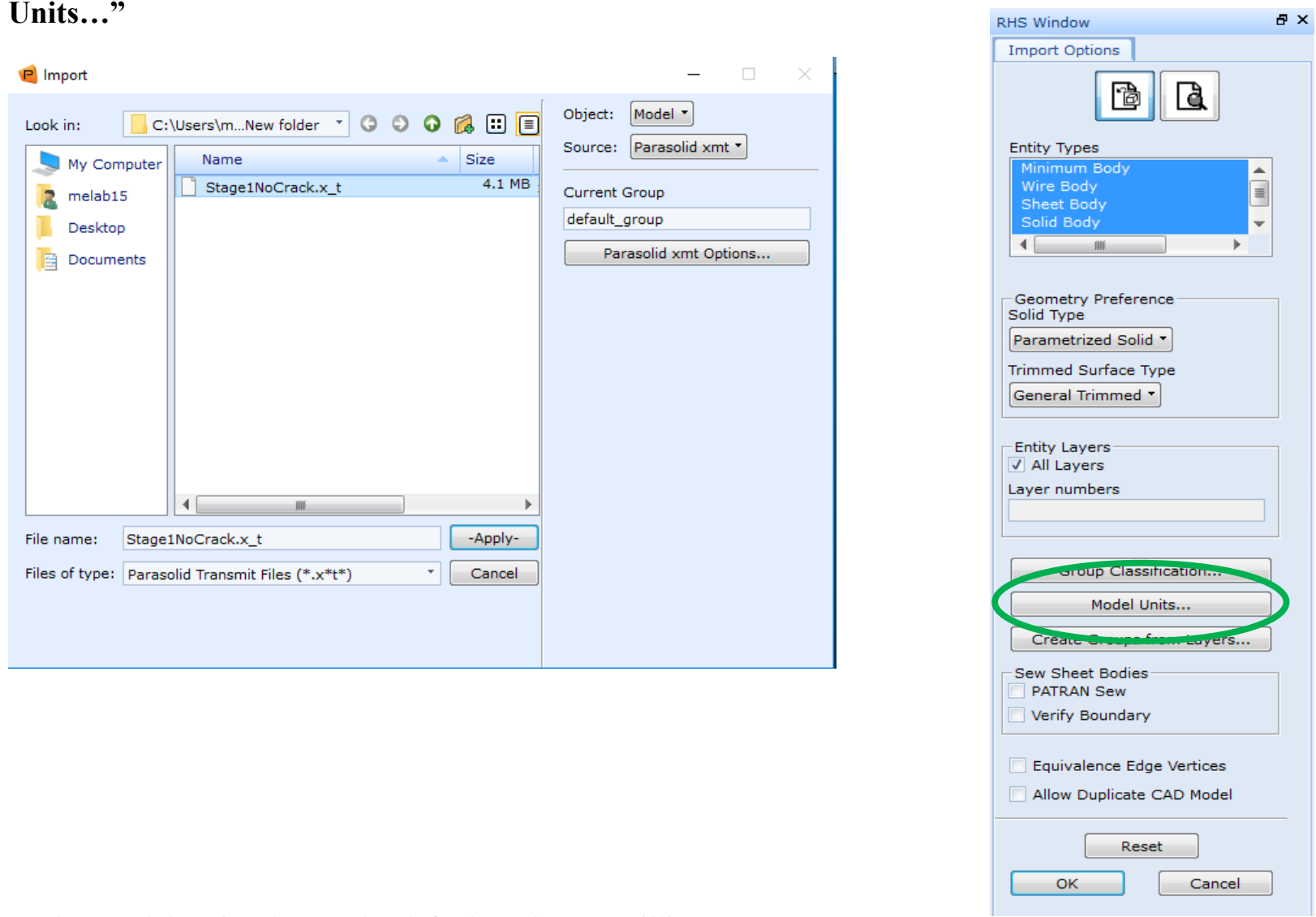

Under Model Units change the default Inches to Millimeters

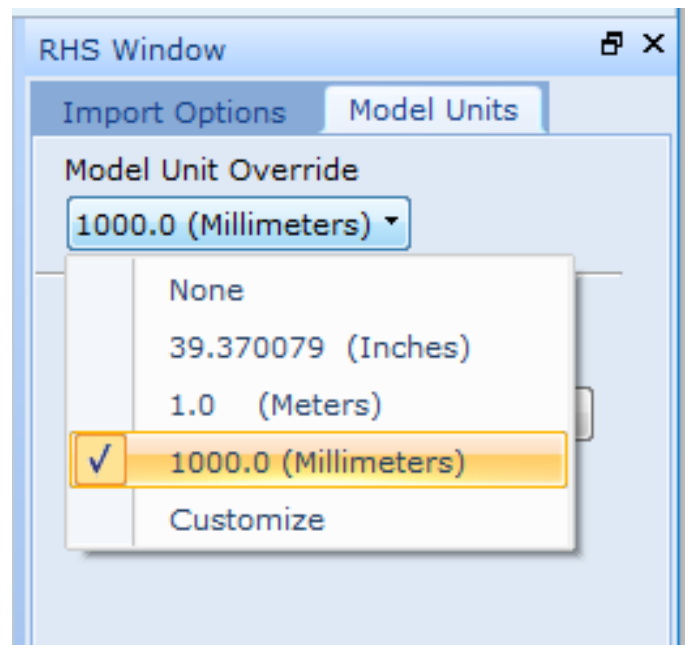

Click "OK" this will bring up a question to confirm the change from inches to millimeters, click "Yes" to return to the Import options window and click "OK" then "Apply". This will import in the parasolid assembly that we have made. 
Once the model is import, a confirmation window will pop up. Click "OK" to accept the imported assembly.

The model will import as a wireframe model. Navigate to the top ribbon under the "Display" section change the view to "Smooth Shaded".
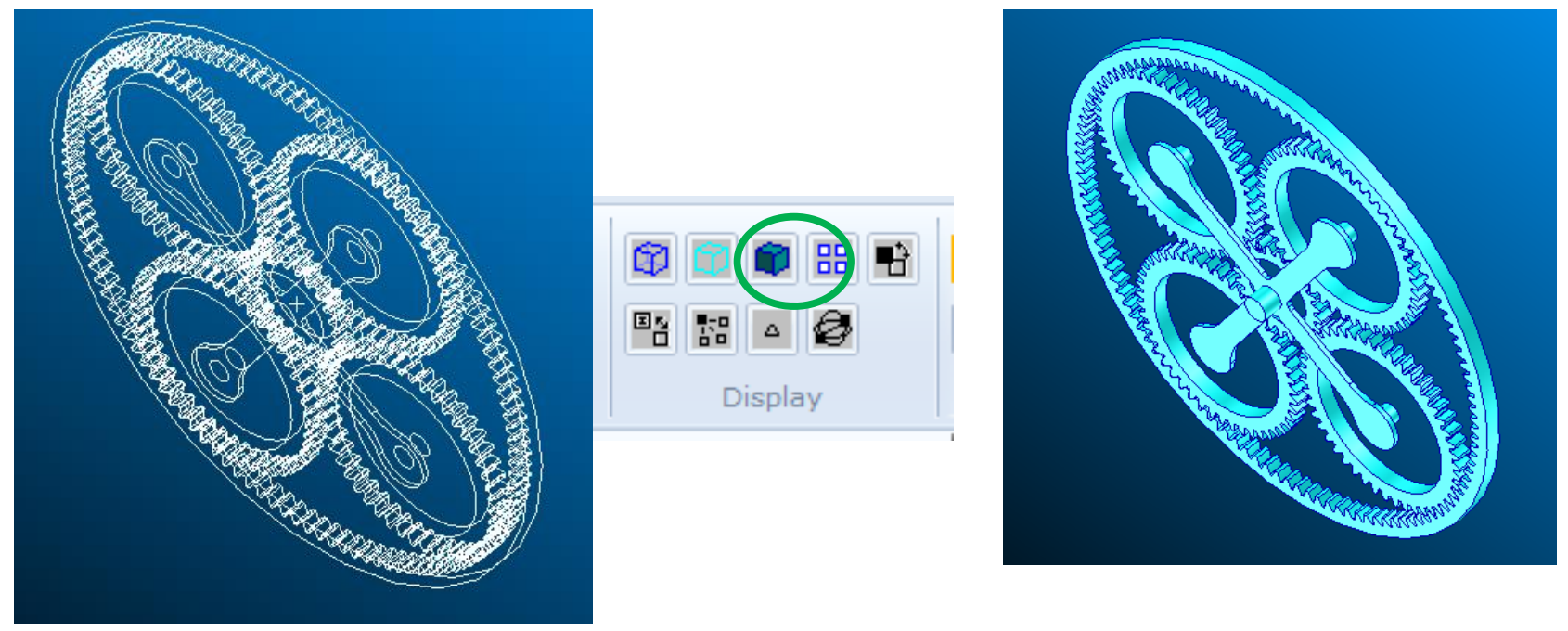

From here click the "Model Tree" button on the top ribbon to bring up the model tree on the left hand side (LHS) of the main view window. Under the "Groups" family in the model tree expend the group to see the different bodies in the model. Uncheck "default_group" and check "SunStage2"

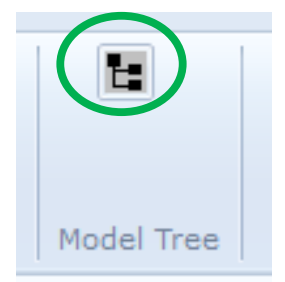

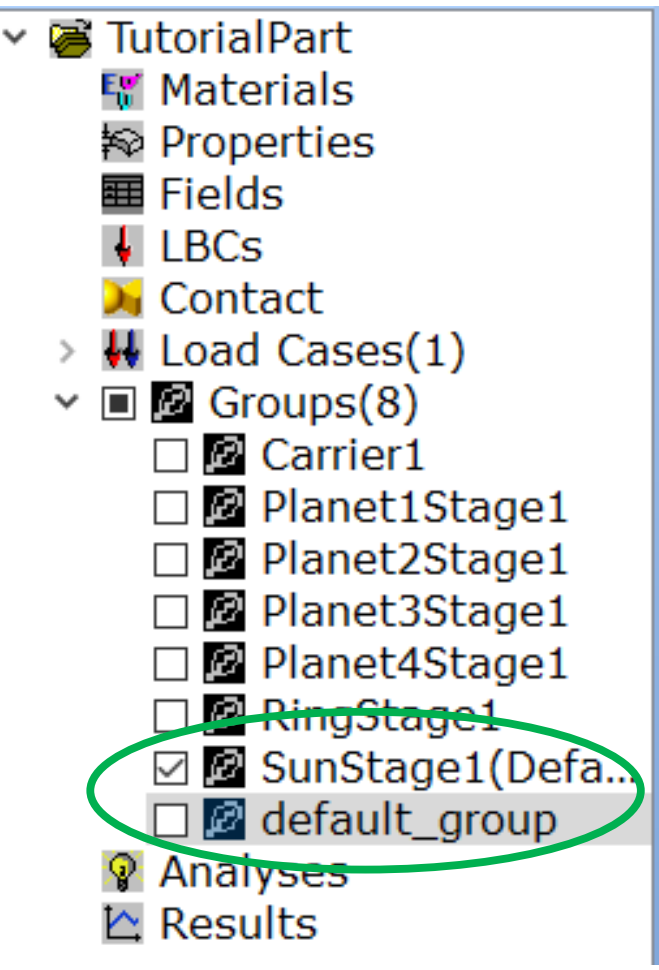


This will isolate only the SunStage1 gear. The model viewer should now only have one part in selected and in view.

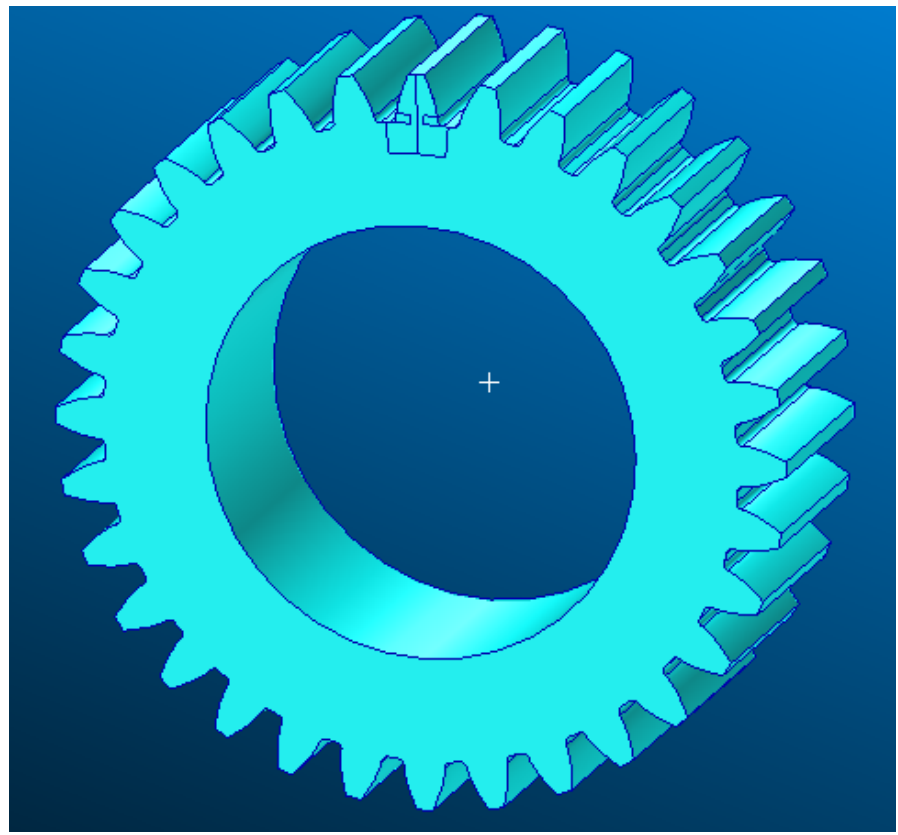

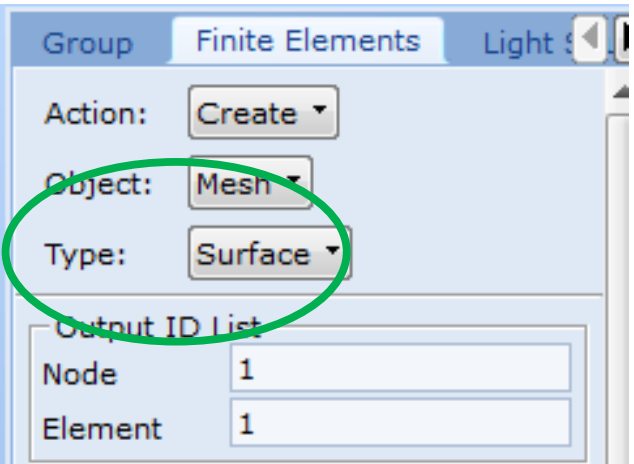
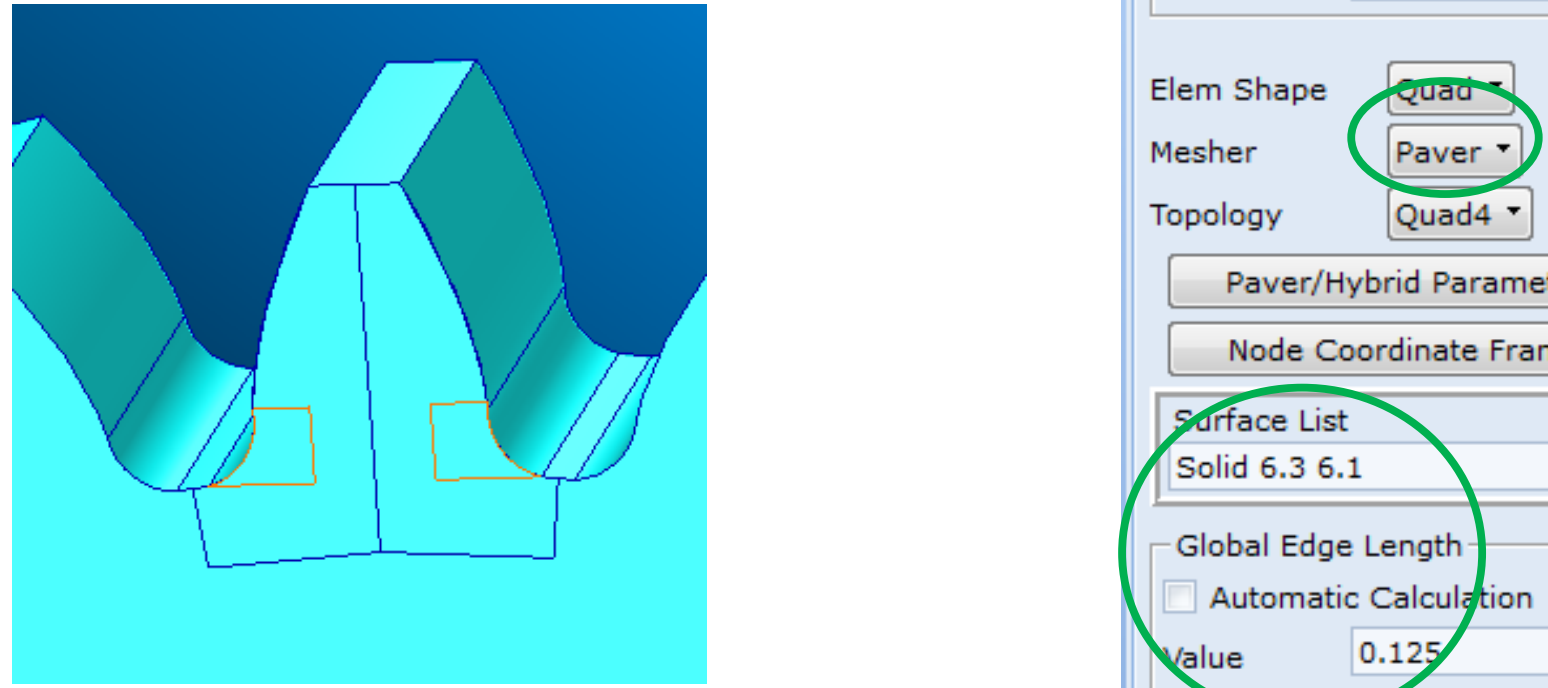

Paver/Hybrid Parameters...

Node Coordinate Frames...

To create the Mesh for the part, at the top ribbon select "Meshing". The Finite Elements menu will appear in the RHS. In this menu select, "Mesh" and "Surface" under the Object and Type drop down menu.

Move down to "Surface List" and select the partitioned root face in the viewport by using shift click to select multiple surfaces. "Solid 6.3 6.1" should show up in the input box. Under "Global Edge Length" uncheck Automatic Calculation and change the Value to “0.125”. Click “Apply". 


\begin{tabular}{cccc} 
Element Type & $\begin{array}{c}\text { Element Shape } \\
\text { Function }\end{array}$ & Location & $\begin{array}{c}\text { Seed Size } \\
{[\mathrm{mm}]}\end{array}$ \\
\hline & & Root & 0.125 \\
Quadrilateral & Linear & Tooth Body & 0.5 \\
$(2$ Dimensional $)$ & $($ Quad4) & Gear Body & 4 \\
& & Thickness & 5 \\
& & Extrusion & \\
\hline \hline
\end{tabular}

Perform this action for all the front faces of the gear according to the table shown above for mesh parameters.

The meshed is shown below:

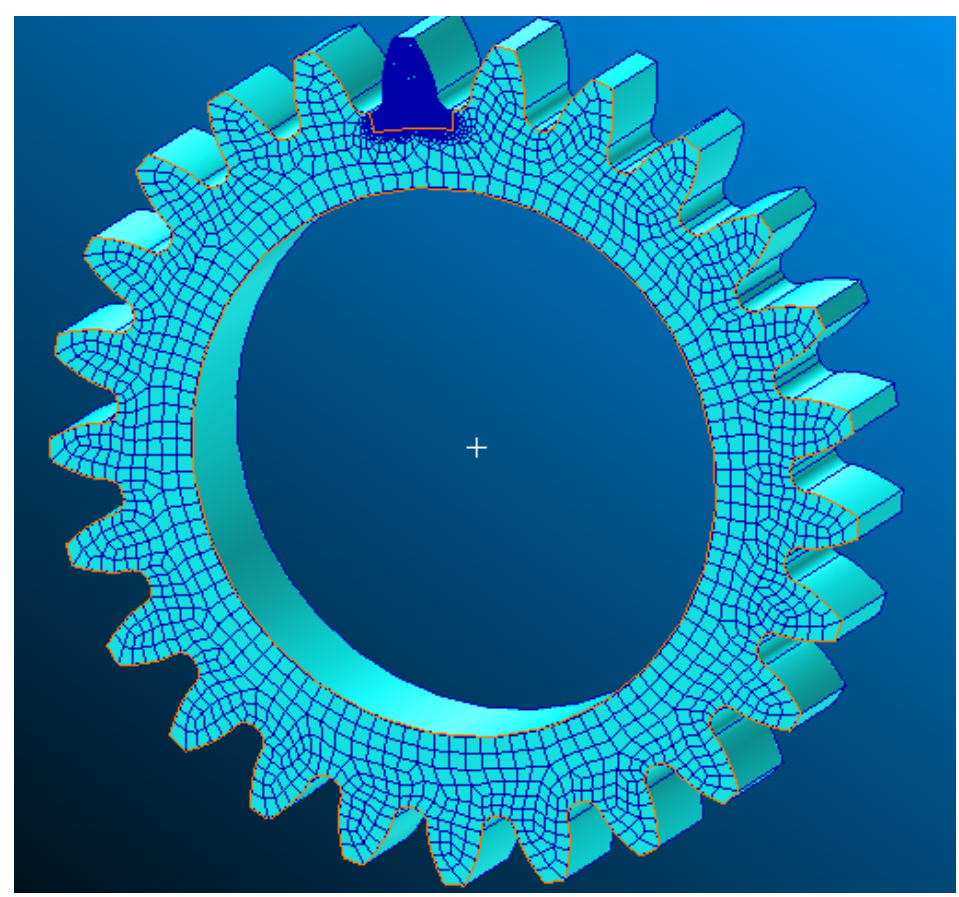

To extrude the mesh to the rest of the body under "Action" select "Sweep" and under "Method" select "Extrude". The direction vector to "<0 0 -1>" and the extrude distance to "5" with and offset of "0.0". 


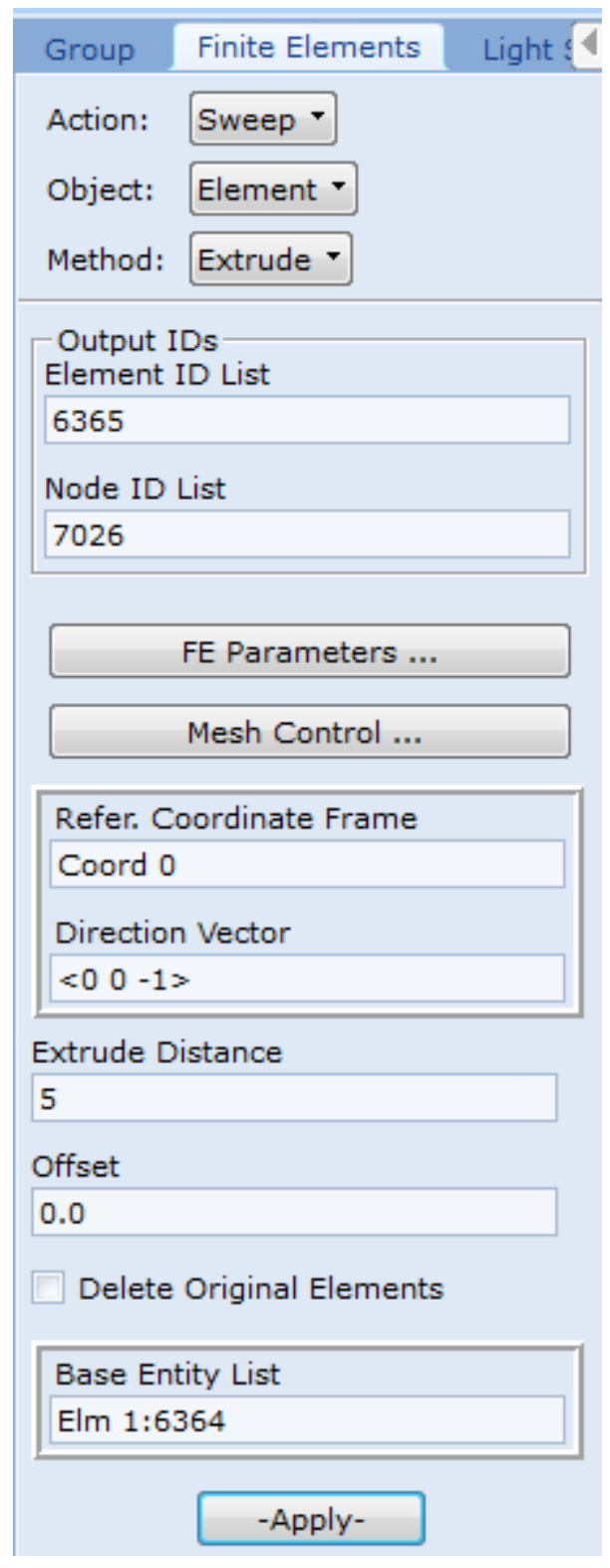

Change the view port to the model front view and highlight all the elements on the face that was just created with the surface mesh. And Click "Apply".

Repeat this method by changing the offset to $5,10,15 \ldots$ etc until the elements are extruded the width of the part. 


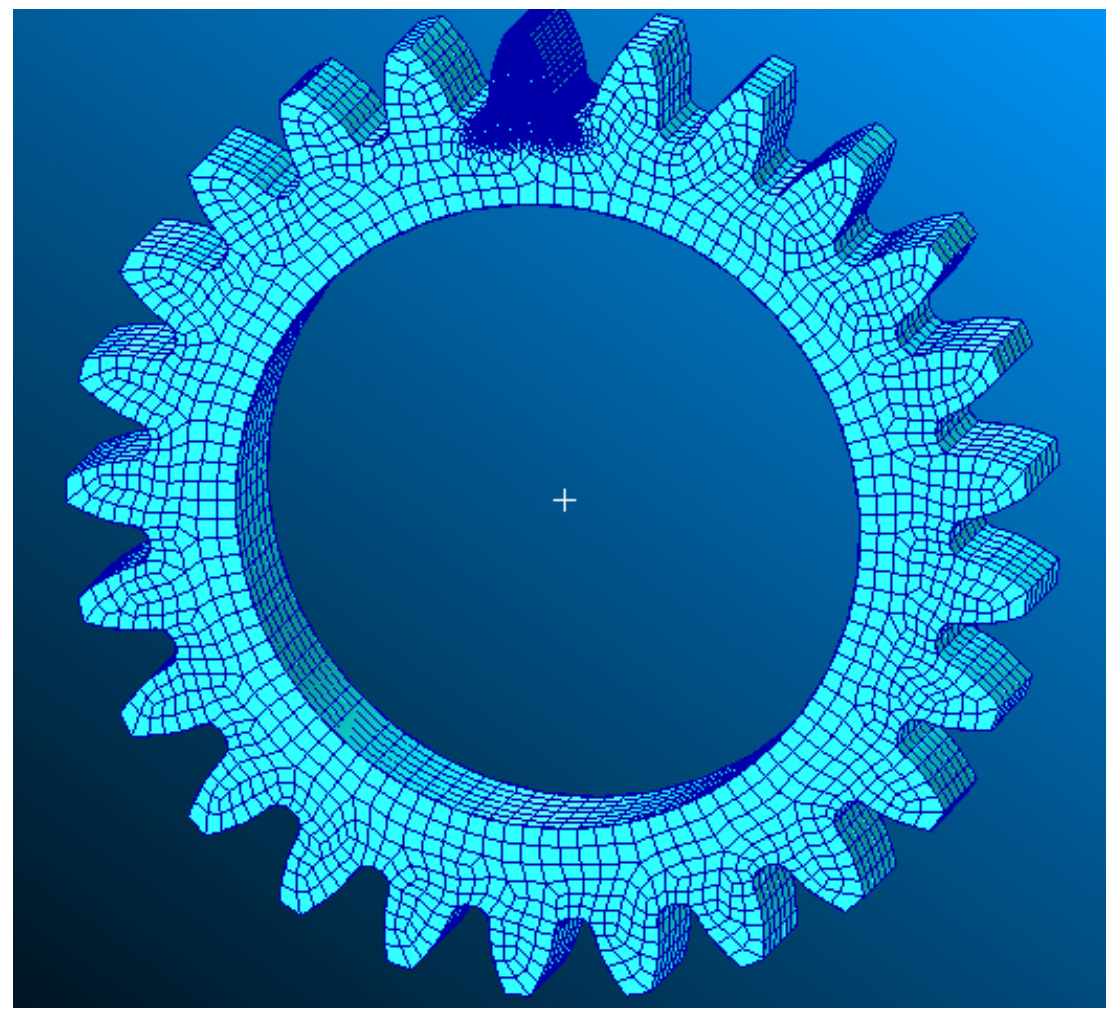

Next, under "Action" pick "Equivalence". Equivalence will eliminate duplicate nodes created in the meshing process.

Leave all the defaults and click "Apply". In the Command Output window a line will appear that indicates the number of deleted nodes.

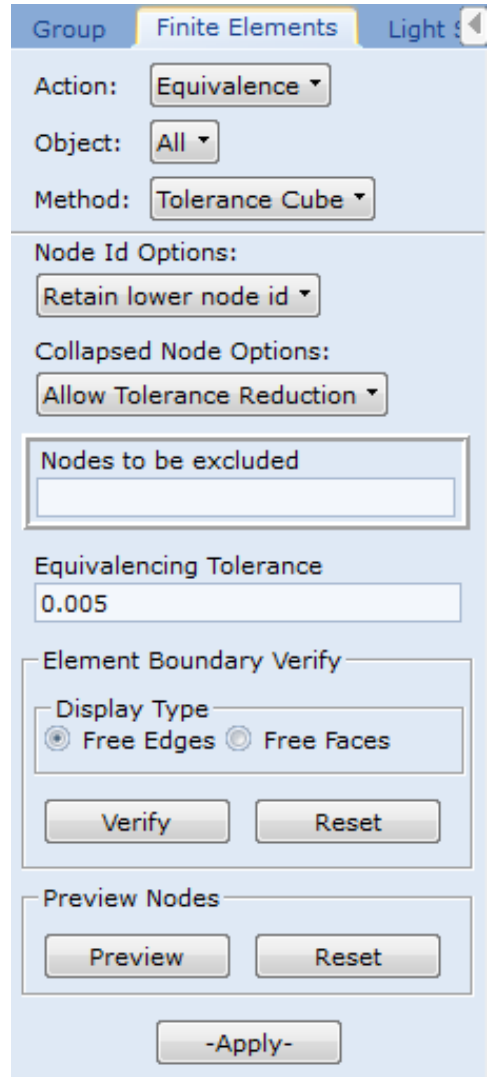

* valauase reallymilmil cumpleteu.

\$\# Geometric equivalencing completed. 16500 nodes deleted.

Next, we will define the material for the part. Navigate to the "Properties" tab at the top ribbon and select "Isotropic" material.

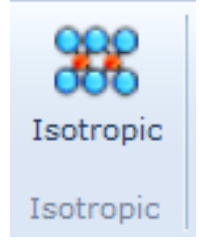

An RHS menu will appear. Under the "Material Name" enter "Steel". This can be the name of the material of the part instead but it is not necessary since we will only be creating one material, any name will work. Then click on "Input Properties..." 


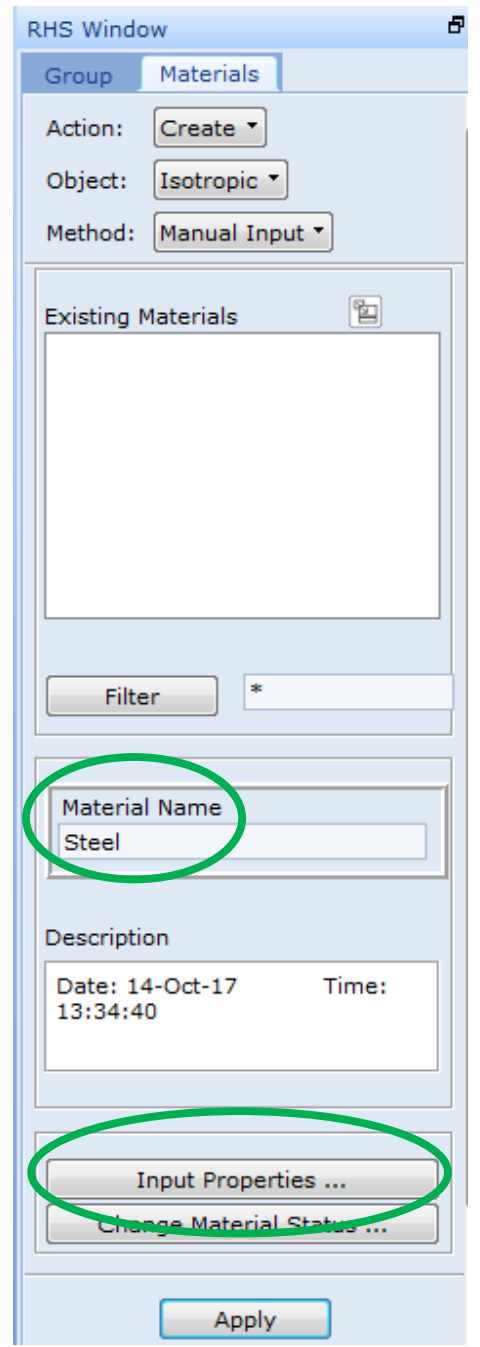

A new window will pop up named Input Options. Here we will enter the material properties of our gear steel. Note that the units must remain consistent. In this case, MMKS. Click "OK". To return to the Materials RHS menu then click "Apply". 
Input Uptions

Constitutive Model:
\begin{tabular}{|l|l|}
\hline Property Name & Linear Elastic - Value \\
\hline Elastic Modulus $=$ & 210000 \\
Poisson Ratio $=$ & \\
Shear Modulus $=$ & \\
Density $=$ \\
Thermal Expan. Coeff $=$ \\
Structural Damping Coeff $=$ \\
Reference Temperature $=$ \\
\hline
\end{tabular}

Temperature Dep/Model Variable Fields:

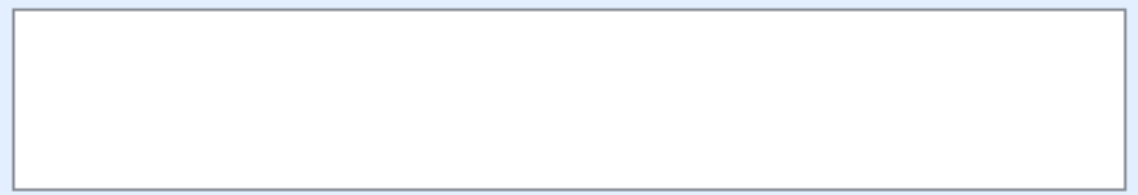

Current Constitutive Models:

\section{OK}

Clear

Cancel

Once the material has been created the material named "Steel" should show up in the LHS model tree.

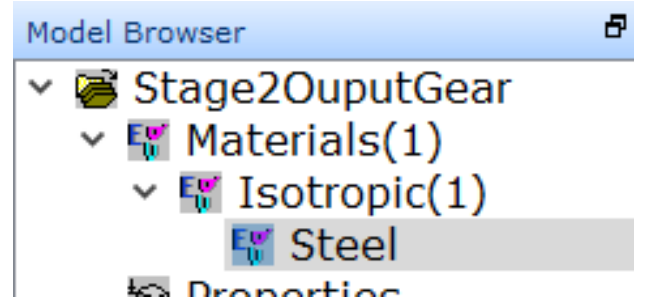

Now we will apply the material to the body. Under the same Properties tab in the top ribbon click "Solid". 


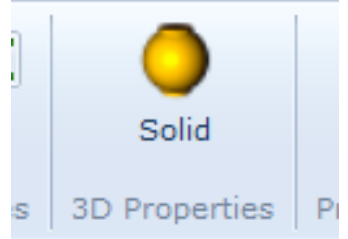

A RHS menu will pop up named Element Properties. Enter a property set name "gearProp" then click on Input Properties.

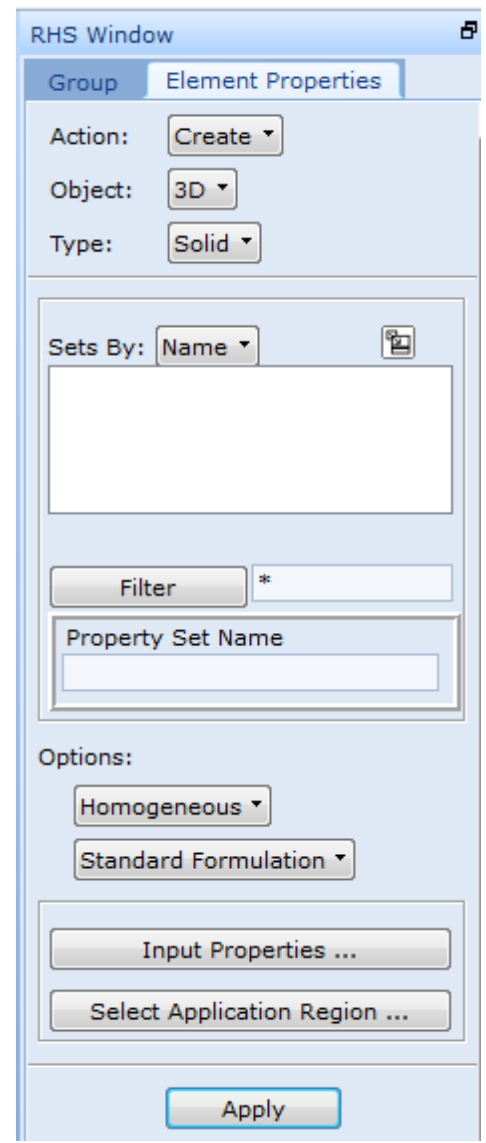

A new window will appear: 


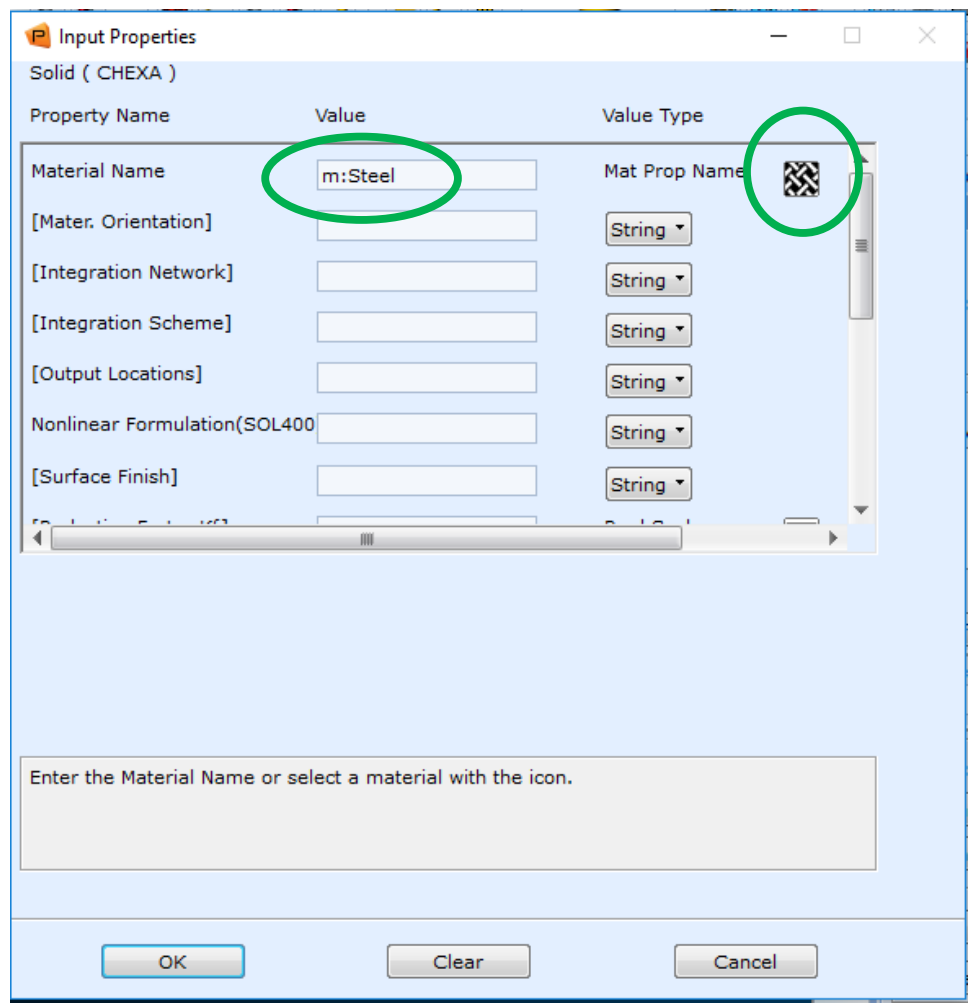

Click on the square symbol marked in the right green circle and select the material we created "Steel". Under Material Name, the box should be filled with "m:Steel" as shown above with the left green circle. Click "OK". This will return the the RHS Element Properties menu. At the bottom click "Select Application Region...".

On the Right tab of the viewport, select "View Solid Elements" .

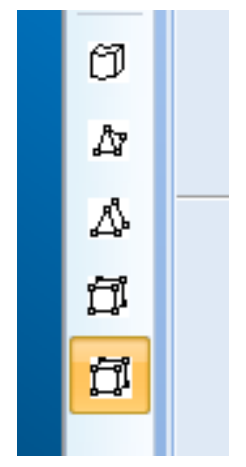


In the new RHS window under select members click the gear in the viewport and Elm 6365:44548 should appear in the text box. Click "Add" this will add it to the Application Region window below then Click "OK" then "Apply".

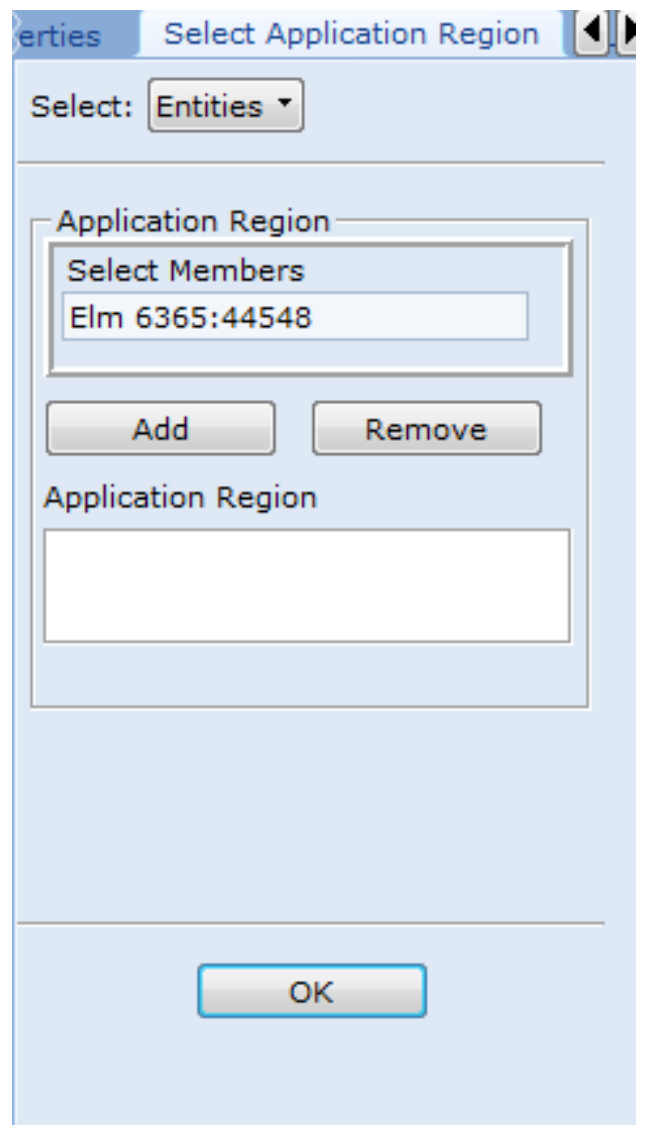

"gearProp" will appear in the Model Tree on the LHS under Properties:

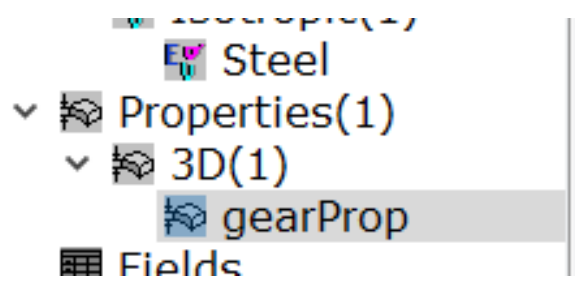

Now we will apply the nodes where this gear is to be constrained, the points where bearings will ideally exist. Under the Home tab on the top ribbon, a wireframe view, erased FEM and large point size will make this process easier.

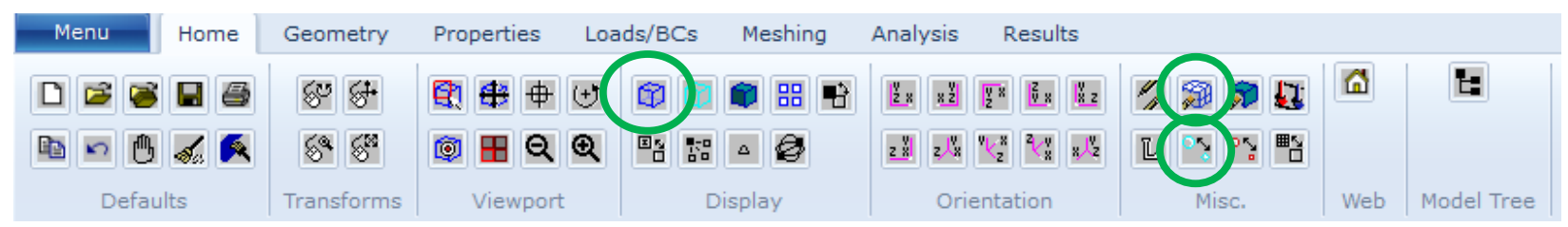

The model in the viewport should look like this after those 3 changes: 


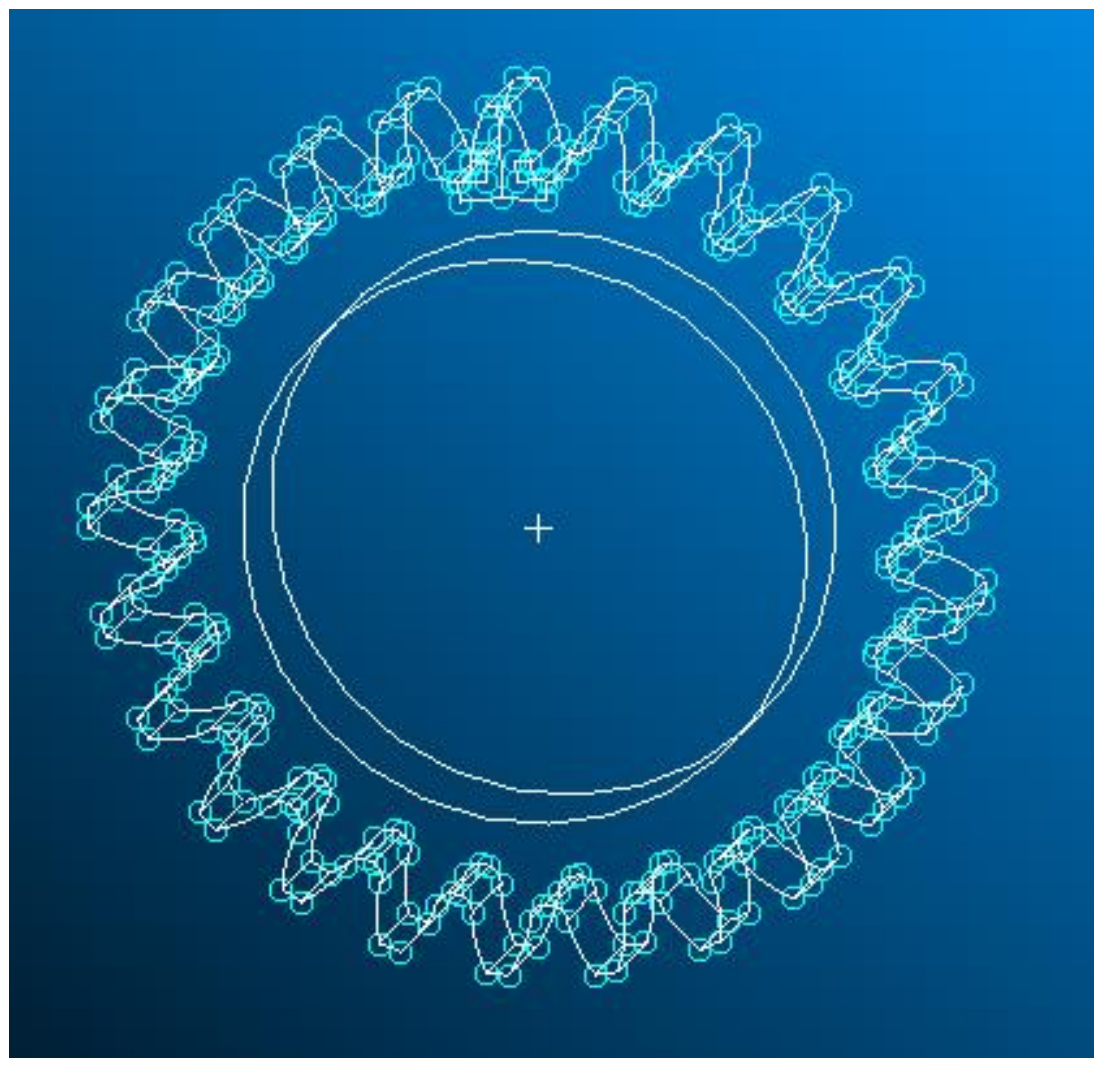

Navigate to the Geometry tab at the top ribbon, a new RHS menu will pop up. Under "Method" change XYZ to "ArcCenter". With Auto Execute checked click on the curve that represents the inside edge of the gear.

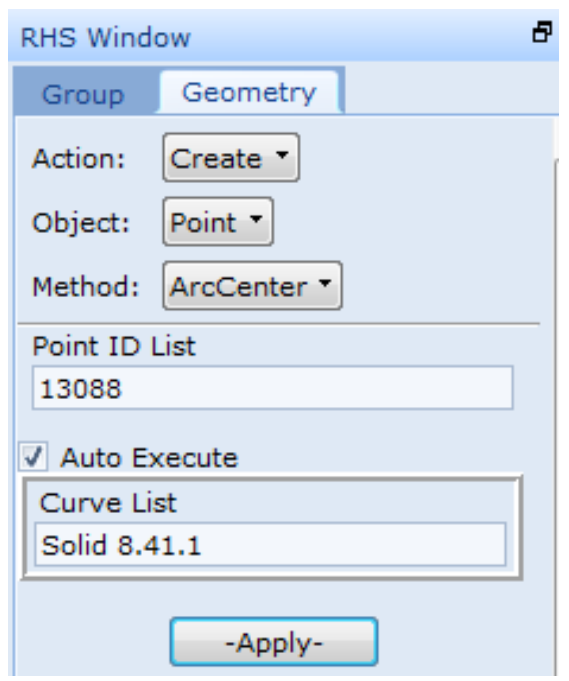

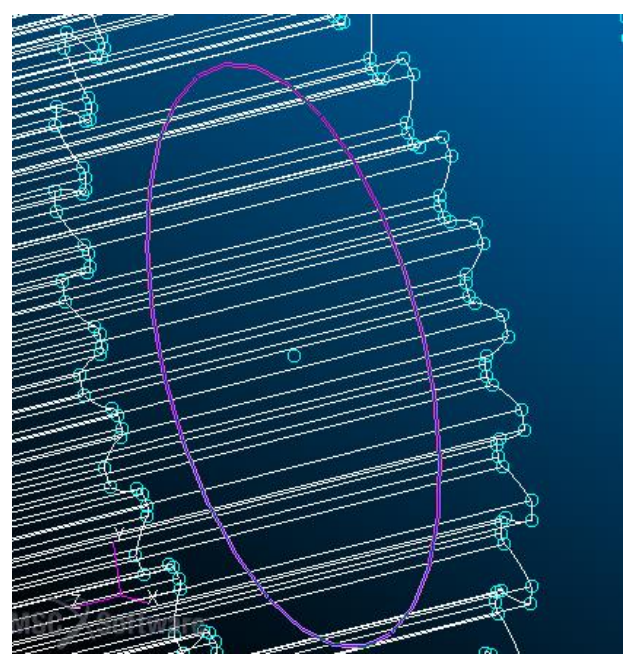

Repeat this step for 3 other locations. The final product should look like this: 


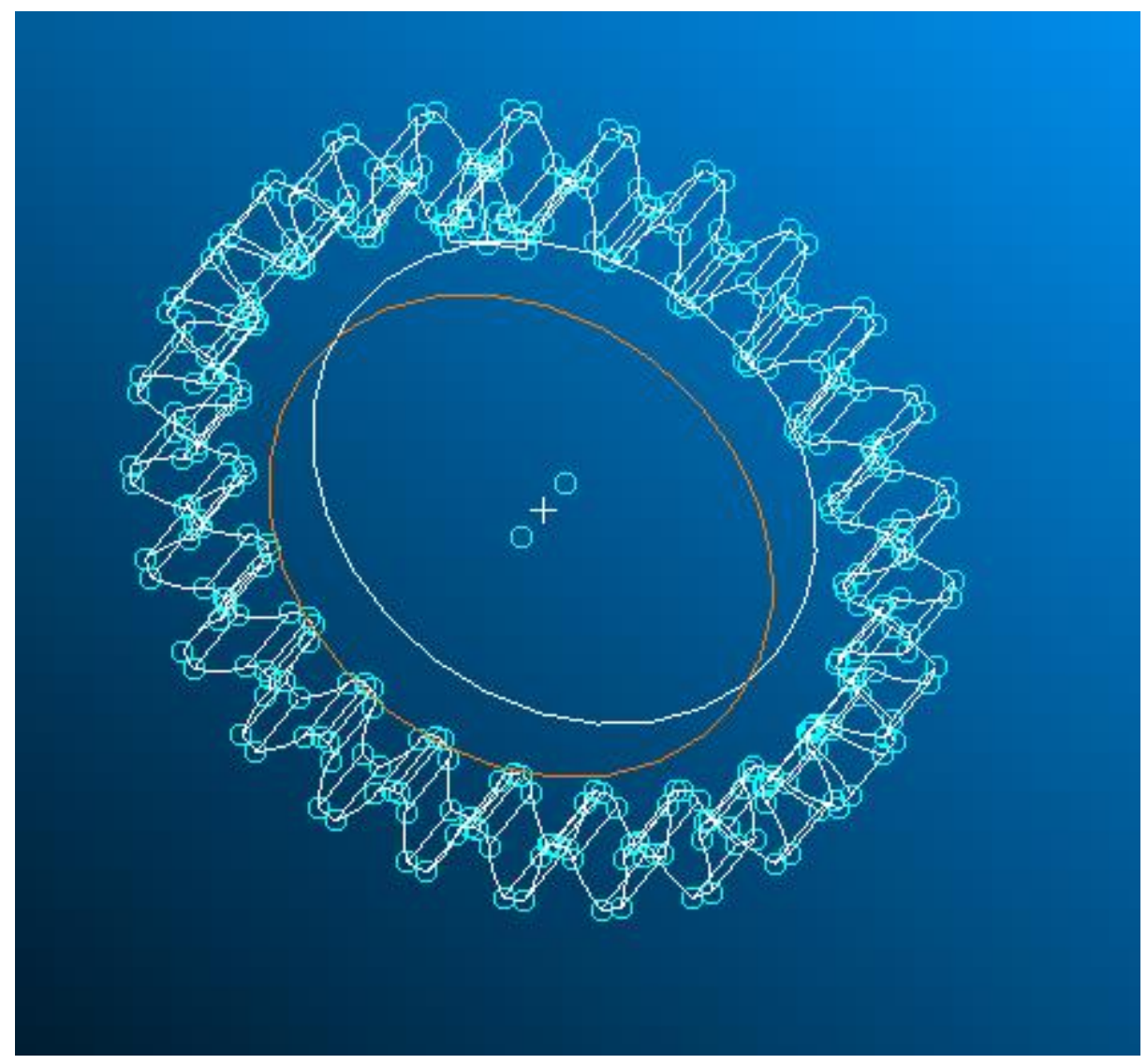

Now, we will interpolate between the points to create a point at the center of the gear. Under "Method" change ArcCenter to "Interpolate". At the bottom, select two of the points created to interpolate between them. 


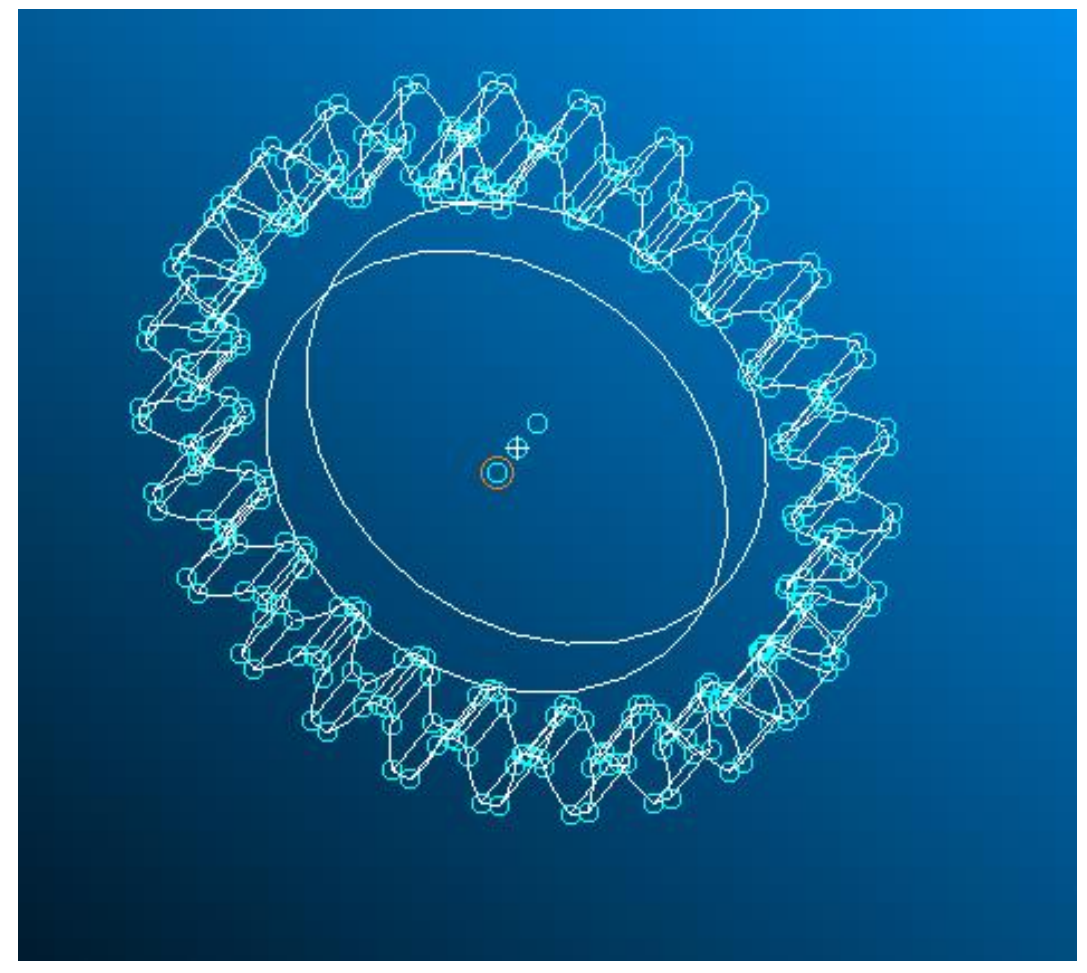

\begin{tabular}{l} 
Group Geometry Light Sources \\
Action: Create \\
Object: Point \\
Method: Interpolate \\
\hline Point ID List \\
\hline 3588 \\
Option: Point \\
Number of Interior Points \\
\hline 1 \\
\hline Point Spacing Method \\
\hline Uniform \\
Nonuniform \\
\hline Point 3585 \\
\hline Manifold to Surface \\
\hline Point 1 List \\
\hline Point 3586 \\
\hline
\end{tabular}


These points will act as reference points. Now that they have been created, under "Menu" $\rightarrow$ "Utilities" select "FEM-General" $\rightarrow$ "RBE2/RBE3 Spider ..."

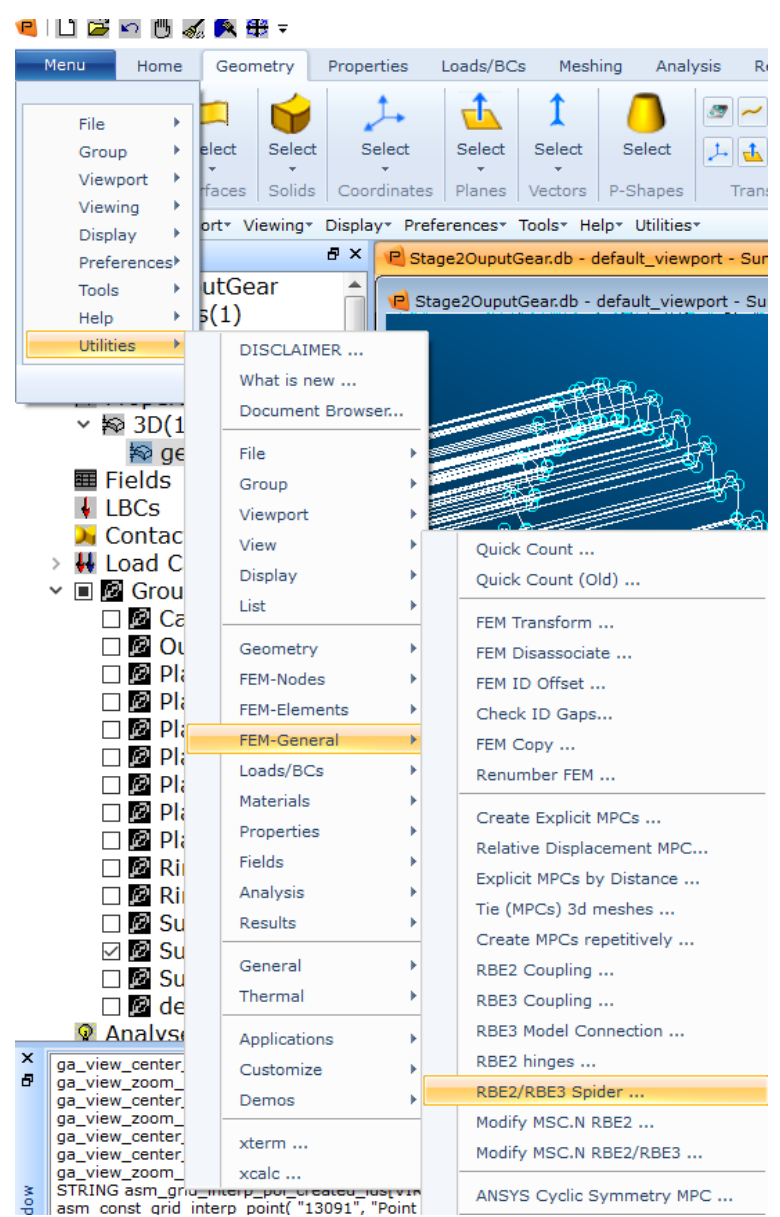


RBE2/RBE3 Spider Menu will pop up on the RHS menu. Here we will select all Dependent DoF. Turn on Plot FEM to see the elements. Under "Method" select "Nodes". At the bottom uncheck "Auto Create Reference Node". Select the text box "Ref Node Location" click on the center point of the gear. Then select the "Nodes" text box and control click to highlight all the inside nodes of the gear body, as shown below. Click "Apply" to generate the RBE2 Spider from the inside nodes to the center of the gear.

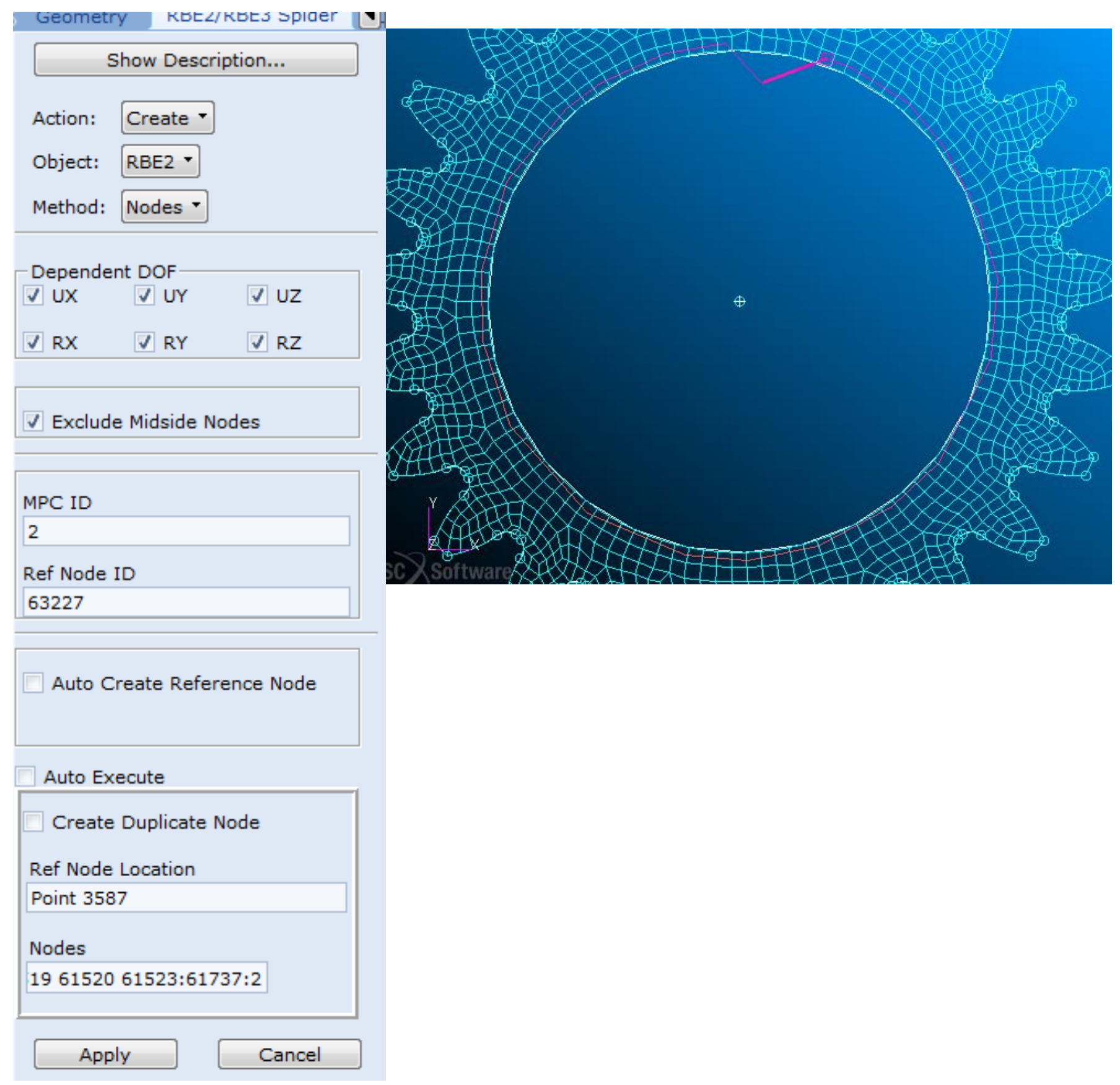


Once completed the model should look like this:

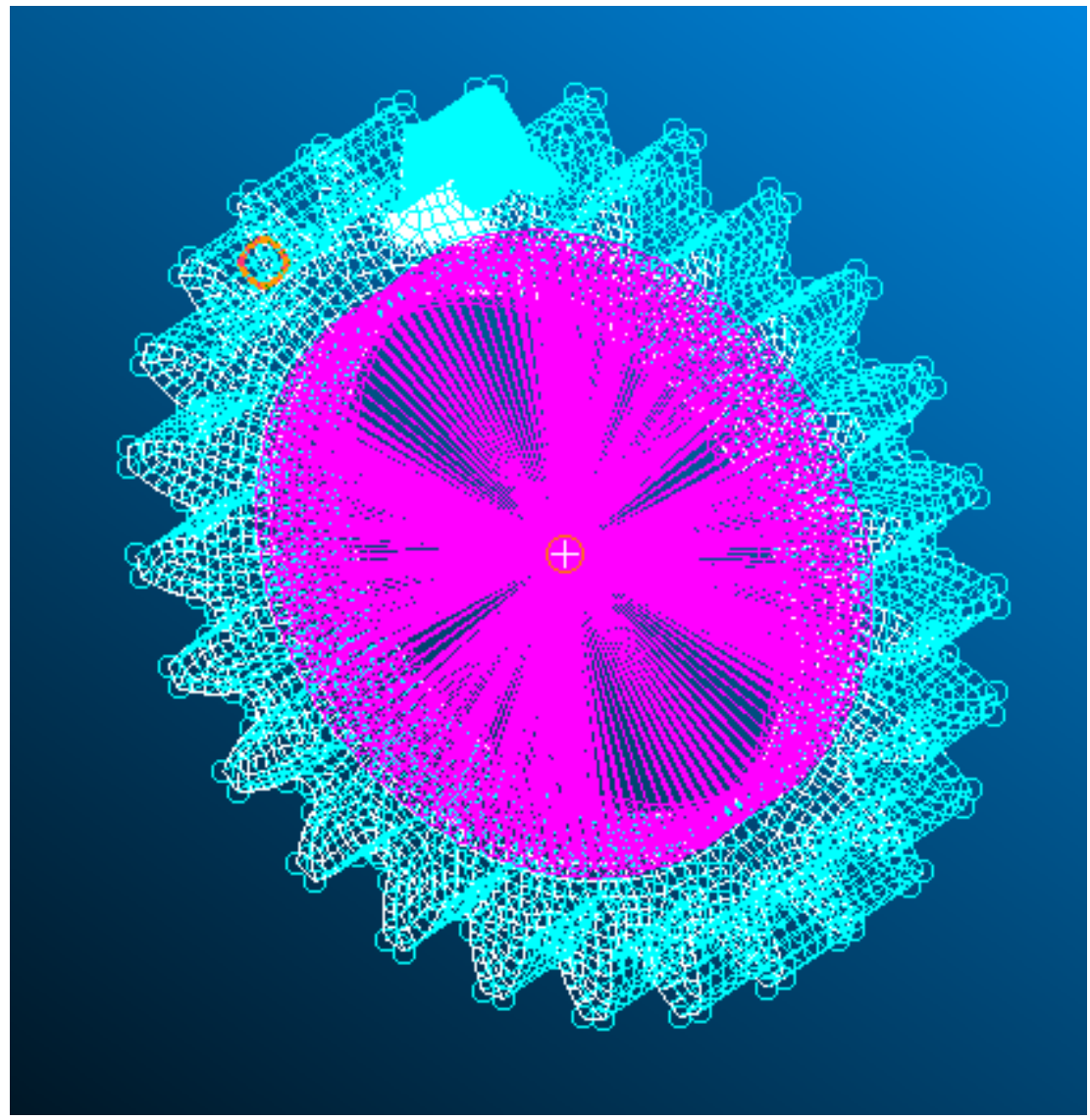

Now we must create a Degree of Freedom list for each of our reference points. Defining DoF for these references point will indicate to the Modal Neutral File (.mnf) file that these points are attachment points. This is called creating a super element in Adams with these nodes as super elements nodes.

Navigate to the "Meshing" tab at the top ribbon. Under the RHS Finite Elements Menu, change the "Object" from Mesh to "DOF list". Under the "DoF List Name" text box type in "gearDoF". This will be the name of our DoF List. Click on "Define Terms..." 

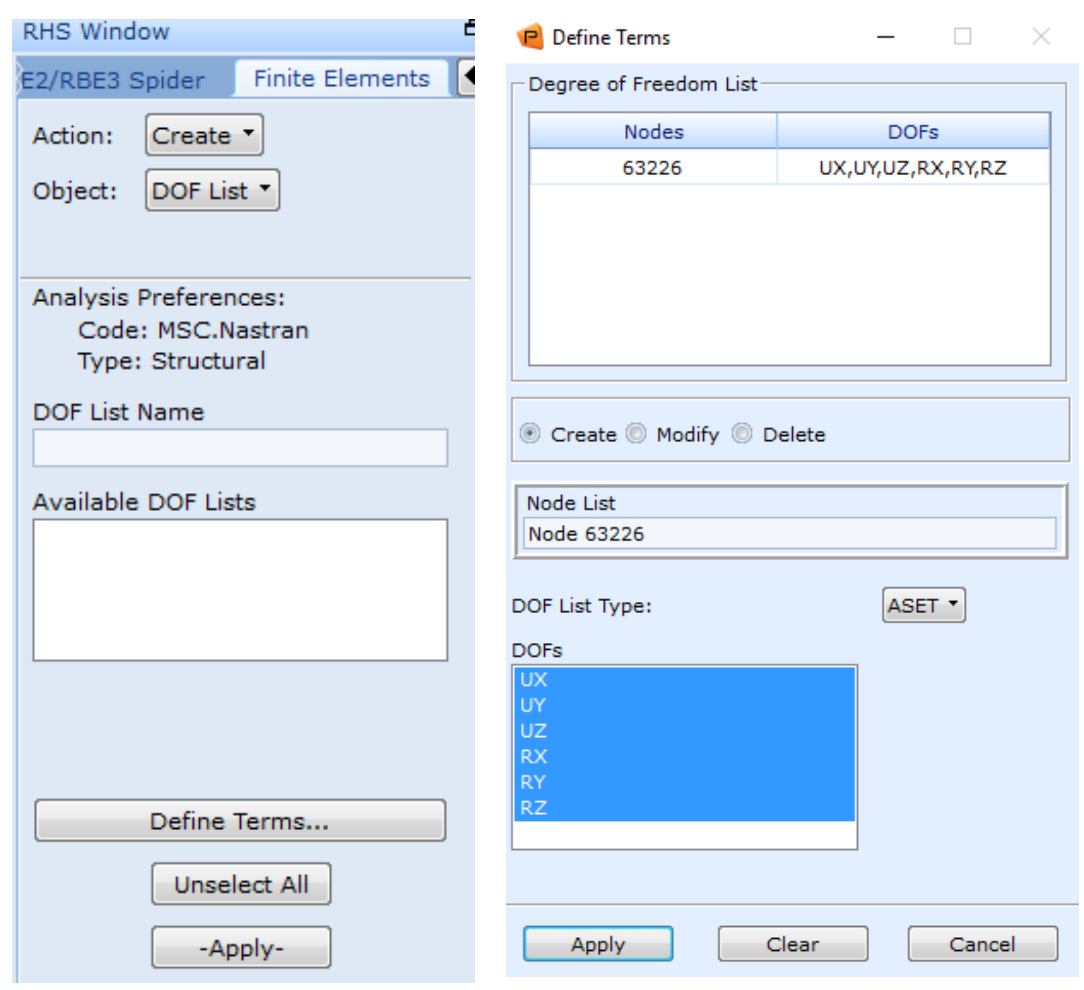

A new window will pop up called Define Terms. Here, we will input one of our two nodes under the "Node List" text box and highlight all DOFs and click "Apply". Repeat this process for the other node. These two nodes will appear in the top box.

Once this step is complete the model set up is finished. Now the output and analysis parameters have to be changed so that the solution can be made compatible with Adams.

Return back to the top ribbon and select on "Analysis". 
Under the Analysis tab, click on "Solution Type". For the output, we will want the normal modes of the gear. Select "Normal Modes" click on "Solution Parameters". A new window should pop up. In this window, click on "ADAMS Preparation".

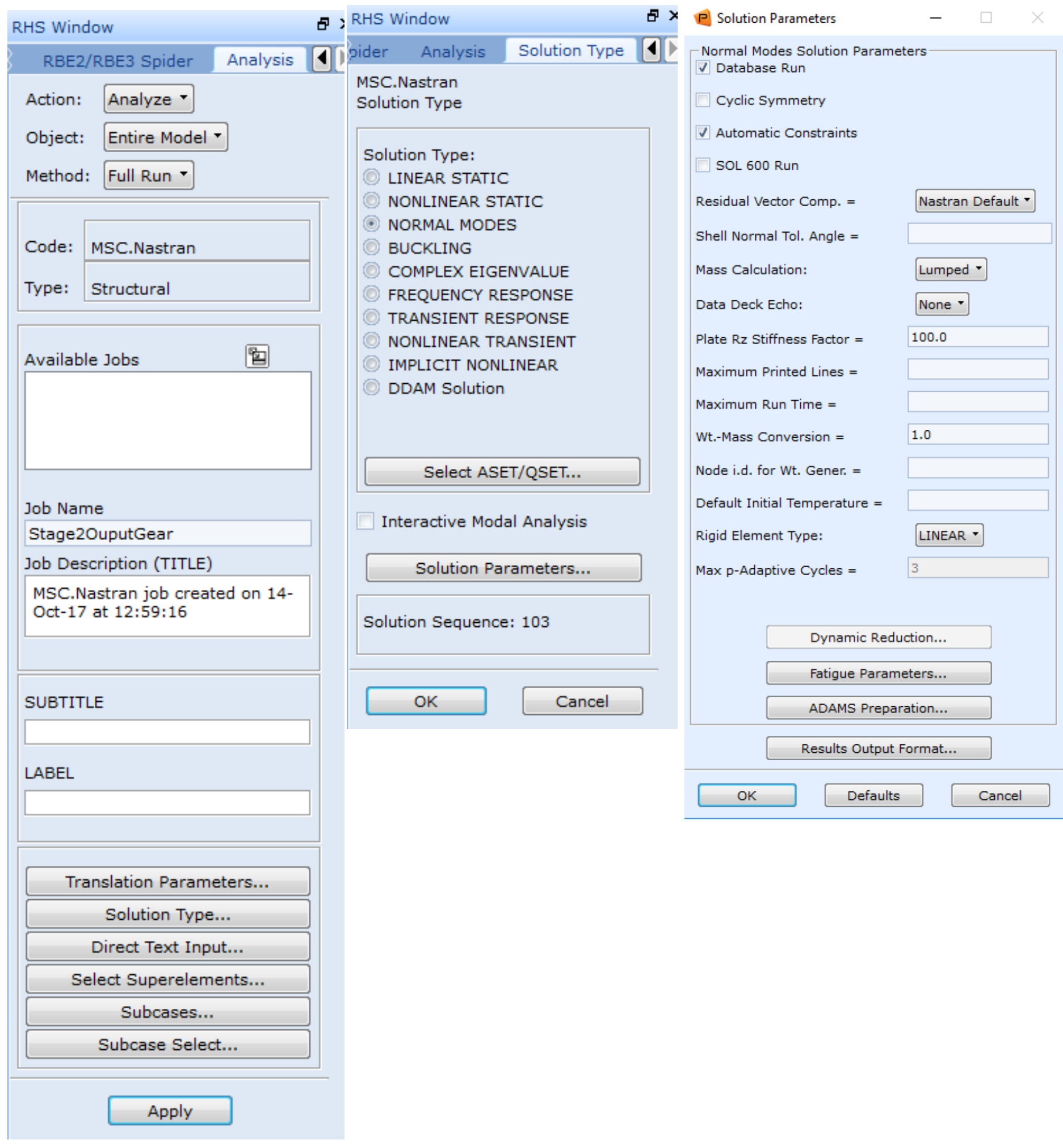


Once inside of ADAMS Preparation, use the drop down menu to change the Adams Output from None to "Full Run+ MNF". The MNF only option is also applicable since this is the file that is required to integrate into ADAMS however MNF only will not allow for stress calculations. Click on "Units..." to set the units of the output file to ADAMS. In this case, we will set these units to be millimeters, kilograms, newtons, seconds. Change the "Num. Shapes to Adams" to "40" Note that this does not set the units for the part in Patran. If importing flexible bodies into ADAMS causes scale of parts then refer back to the beginning of the tutorial for import parasolids into Patran. Note that some types of models me require more mode shapes than others to describe the whole story of the part.

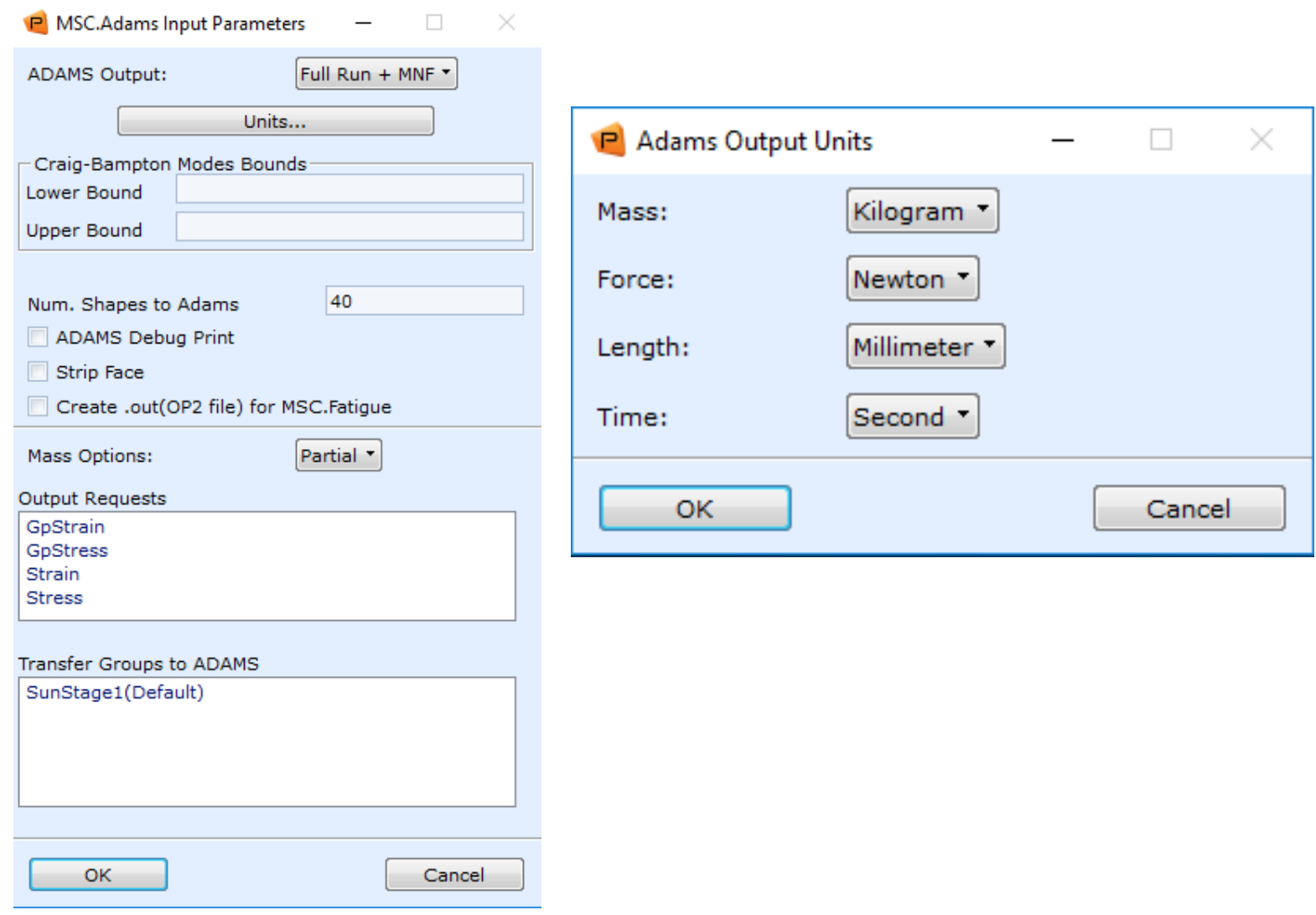

Accept the settings by clicking “OK", “OK” accept pop up messages window by click "OK”, “OK” then "OK", "OK" once more to the Analysis tab.

In the Analysis tab, we will then go to the "Subcases..." menu. Then click "Subcase Parameters...". Under Subcase Parameters change the "Number of Desired Roots =" to match the number to ADAMS output, 40 roots. Once done click “OK”. 
Subcases

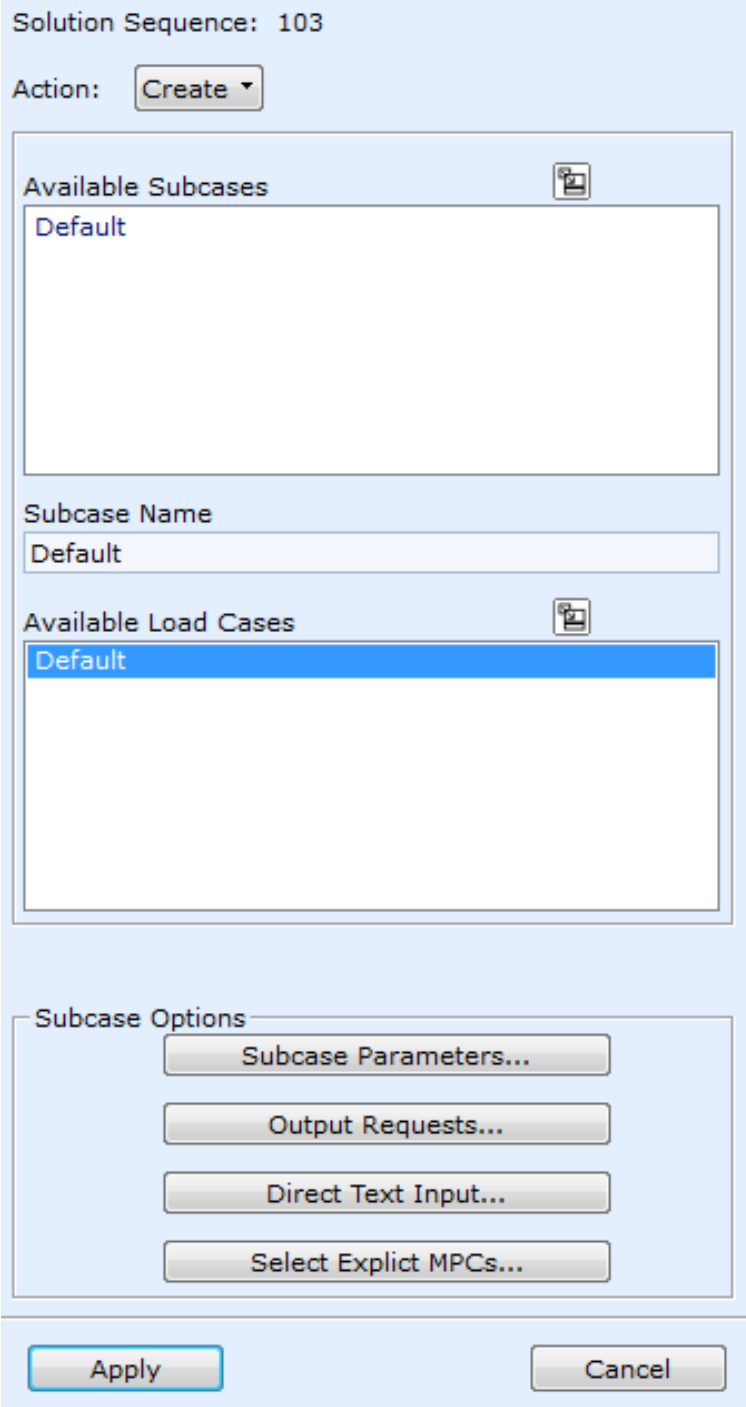

Subcase Parameters

REAL EIGENVALUE EXTRACTION

Extraction Method: Lanczos -

Frequency Range of Interest

Lower $=$

Upper =

Estimated Number of Roots =

100

Number of Desired Roots =

40

Diagnostic Output Level:

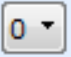

-Results Normalization Normalization Method:

Mass -

Normalization Point $=$

Normalization Component:

$1+$

Number of Modes in Error Analysis =

10

Default Load Temperature =

Use Contact Table

\section{Contact Table...}

\section{OK}

Cancel

For stress outputs in ADAMS click on "Output" request and select "Grid Point Stresses" in the Select Result Type. 


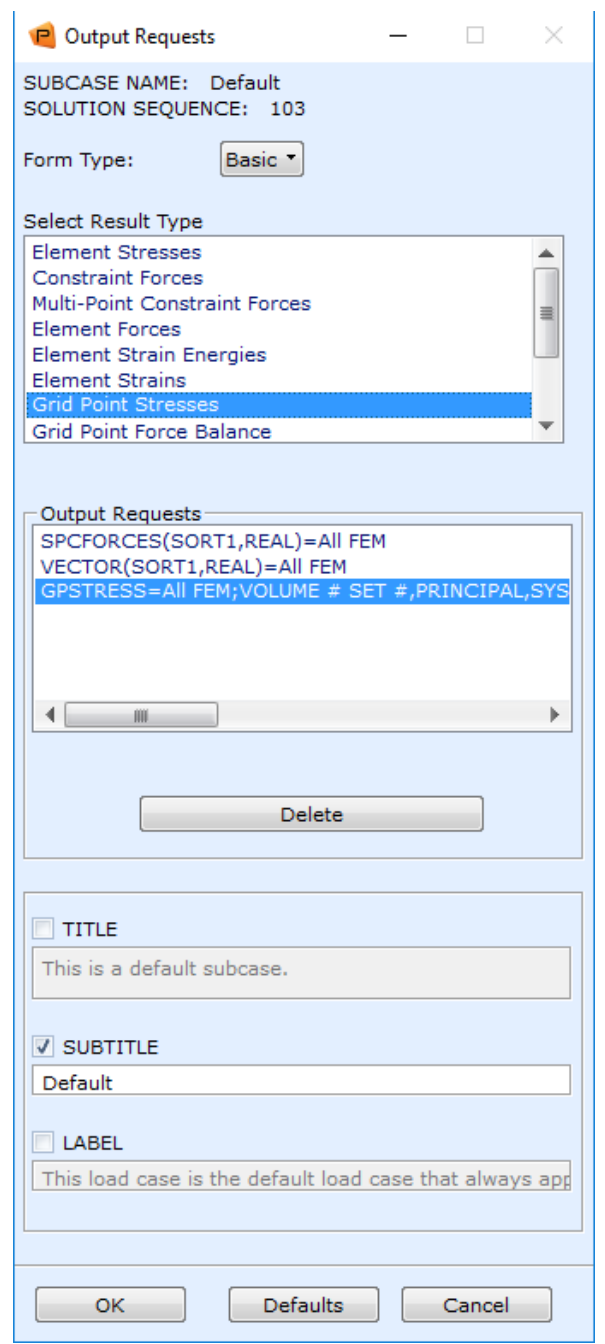

Click "OK" then "Apply" then "Cancel" to exit out of subcase menu.

Once this is complete the model is ready for analysis. Click "Apply" to run the model. A warning message will appear, merely click "Yes".

P Message

Question from application APP INTERFACES

Some elements in the model have no types or properties defined. Do you wish to continue with the translation?

A command window should pop up indicating that the database has been sent to Nastran for analysis. Once the analysis is complete the command window will close. 


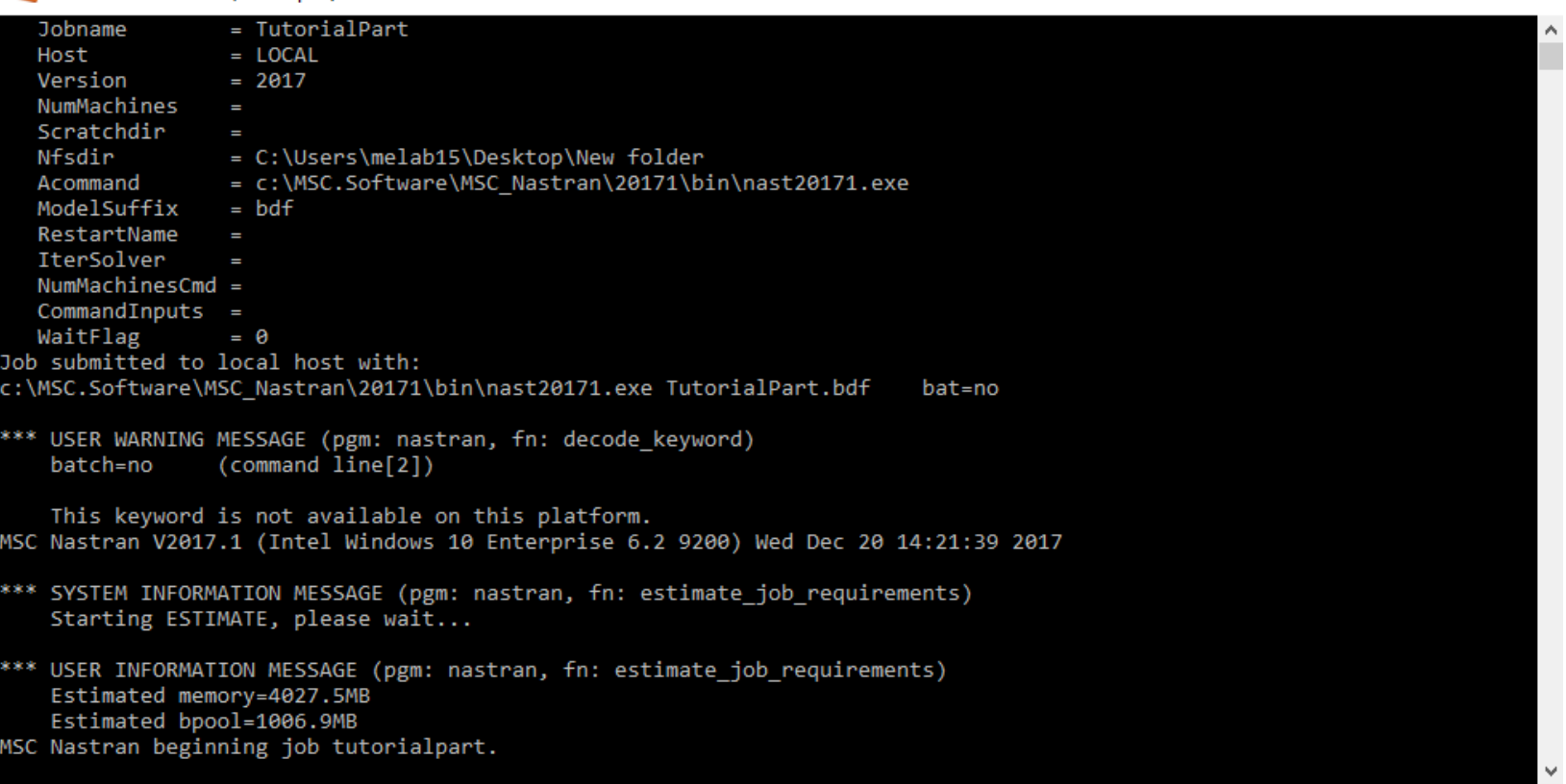

Once the window closes, navigate back to the working directory in windows explorer. There should be a number of files created with the name of the specified job, the one we're looking for is the .mnf file. This is the Patran output file required for ADAMS integration.

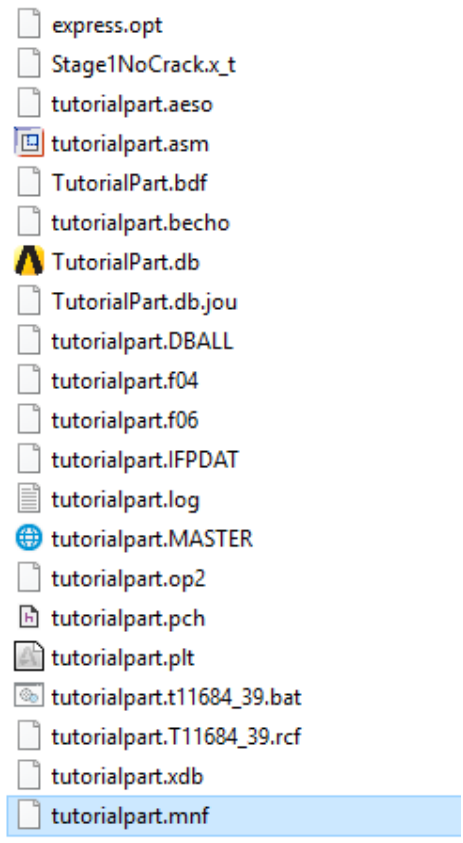

Copy the .mnf file and the parasolid model into a new directory where the ADAMS model will be created. This, again, ensures that all associated files for the system will remain together in one folder.

Start up ADAMS view and select the option to create new a "New Model". Enter a model name, select "No Gravity", set the unit system to "MMKS" (these units should be as whatever was set for ADAMS 
output in Patran). Note it is important to always keep units consistent throughout all the models. Finally select the working directory which was just created with the .mnf file and parasolid assembly.

Create New Model

$\times$

\section{Create New Model}

Model Name Flexible_Model|

Gravity No Gravity

Units MMKS - mm,kg, $\mathrm{N}, \mathrm{s}, \mathrm{deg} \quad$

Working Directory $\longdiv { C \text { :IUsers Imelab15\DesktoplAdamsForTu } }$

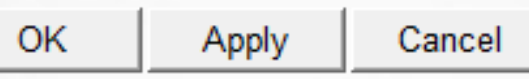

Once the new model has been created. Go to the "Settings" menu and select "Units". This is another check to ensure that we are working in the correct units for our model. Change "Angle" and "Frequency" to "Radian" and "Radian/Second" from Degrees and Hertz.

\begin{tabular}{|c|c|c|c|c|}
\hline \multicolumn{4}{|c|}{4 Units Settings } & \\
\hline Length & \multicolumn{3}{|c|}{ Millimeter } & $\overrightarrow{7}$ \\
\hline Mass & \multicolumn{3}{|c|}{ Kilogram } & \\
\hline Force & \multicolumn{3}{|c|}{ Newton } & $\vec{v}$ \\
\hline Time & \multicolumn{3}{|c|}{ Second } & \\
\hline Angle & \multicolumn{3}{|c|}{ Radian } & \\
\hline Frequency & \multicolumn{4}{|c|}{ Radians/Second } \\
\hline \multirow[t]{2}{*}{ MMKS } & MKS & CGS & & \\
\hline & \multicolumn{3}{|c|}{ OK } & \\
\hline
\end{tabular}

Add a point to ground at the origin. To verify that this point is at ground right click and "Modify". The point should have coordinates $(0,0,0)$.

To import the model into ADAMS, go to "File" $\rightarrow$ "Import". Here we will select the same model that was used to create the flexible body in Patran. Change "File Type" to "Parasolid". In the text box for "File to Read" right click and browse for the parasolid file. This should be the only one in the working directory. Give the model a name and click "OK". 


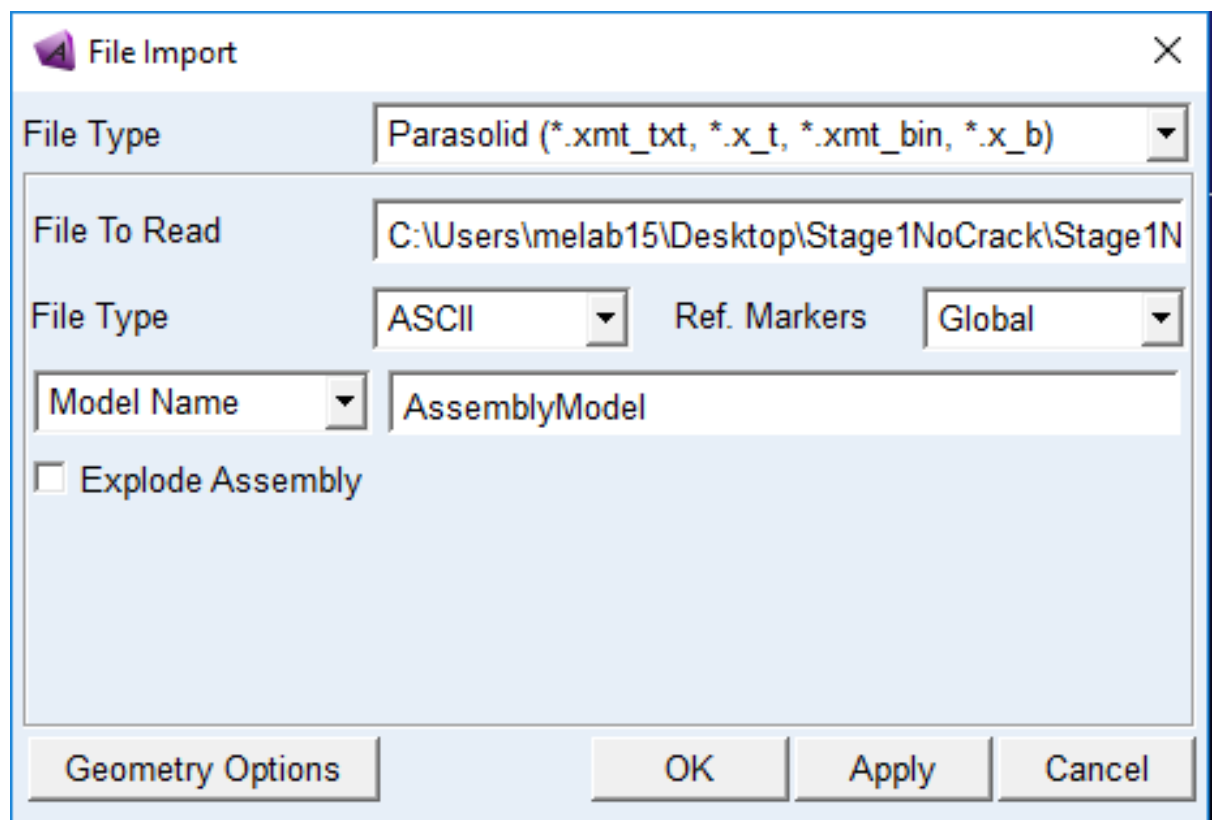

This will import the full model assembly that was created for all three stages in Solidworks. All the unnecessary parts can be removed by going to the model tree on the left and deleting them. Give the rest of the parts a density $\left(7.77 \mathrm{E}-6 \mathrm{~kg} / \mathrm{mm}^{\wedge} 3\right)$. Set initial velocity about the $\mathrm{z}$ axis to 0 .

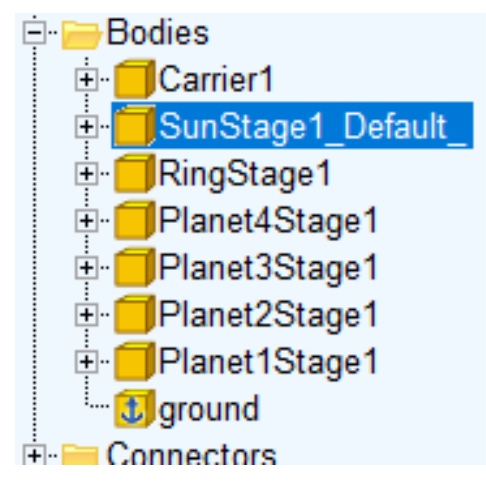

The flexible body in this model will be the SunStage1 which we have just put through Patran. To create flexible model, right click the part on the model tree and click "Make Flexible" and import to import the mnf file for the gear. 


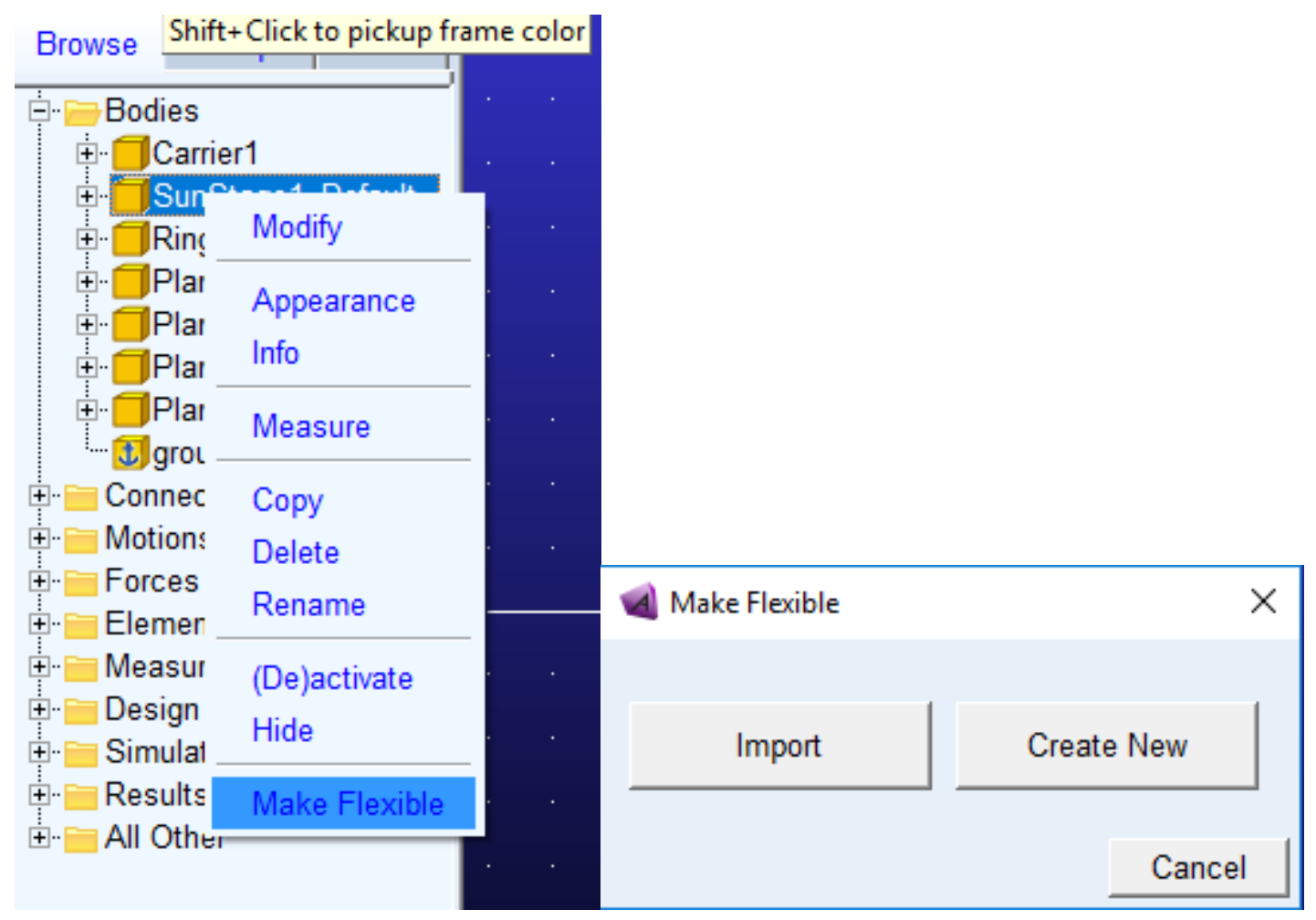

A new window will pop up where the mnf file can be imported into ADAMS. Right click on the blank text box next to the MNF File Drop down menu and click "Browse". Select the .mnf file desired to use for analysis in this case there is only one.

There are options at this point to reposition the new flexible model of SunStage2. However, the part will orient itself in the same location when using the same parasolid model for both ADAMS and Patran. In these case, nothing needs to be done to reposition the part. Click "OK" to import the flexible part.
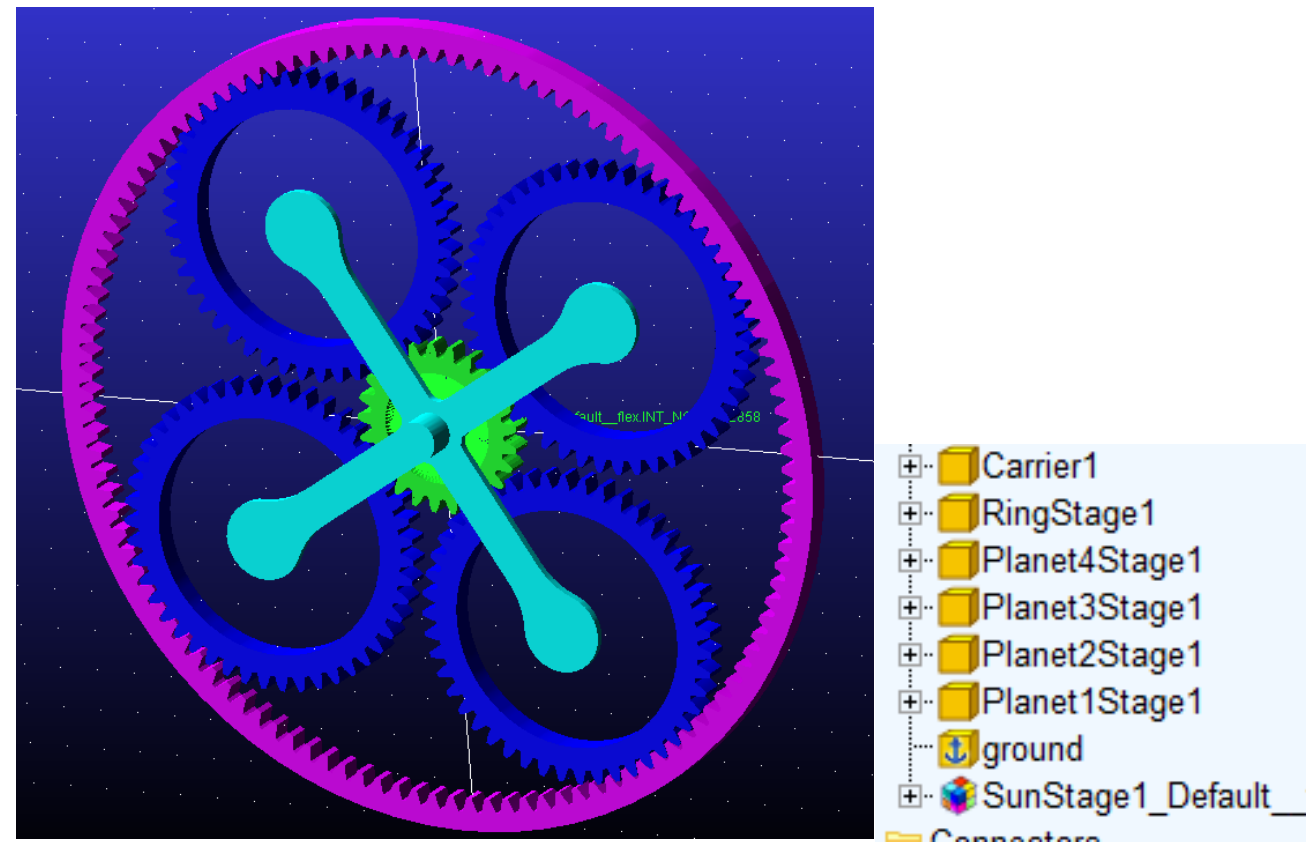
Notice that the model tree has changed with the new part.

Now the model is fully integrated. Add joints to each respective part. Add the appropriate joints for part function.

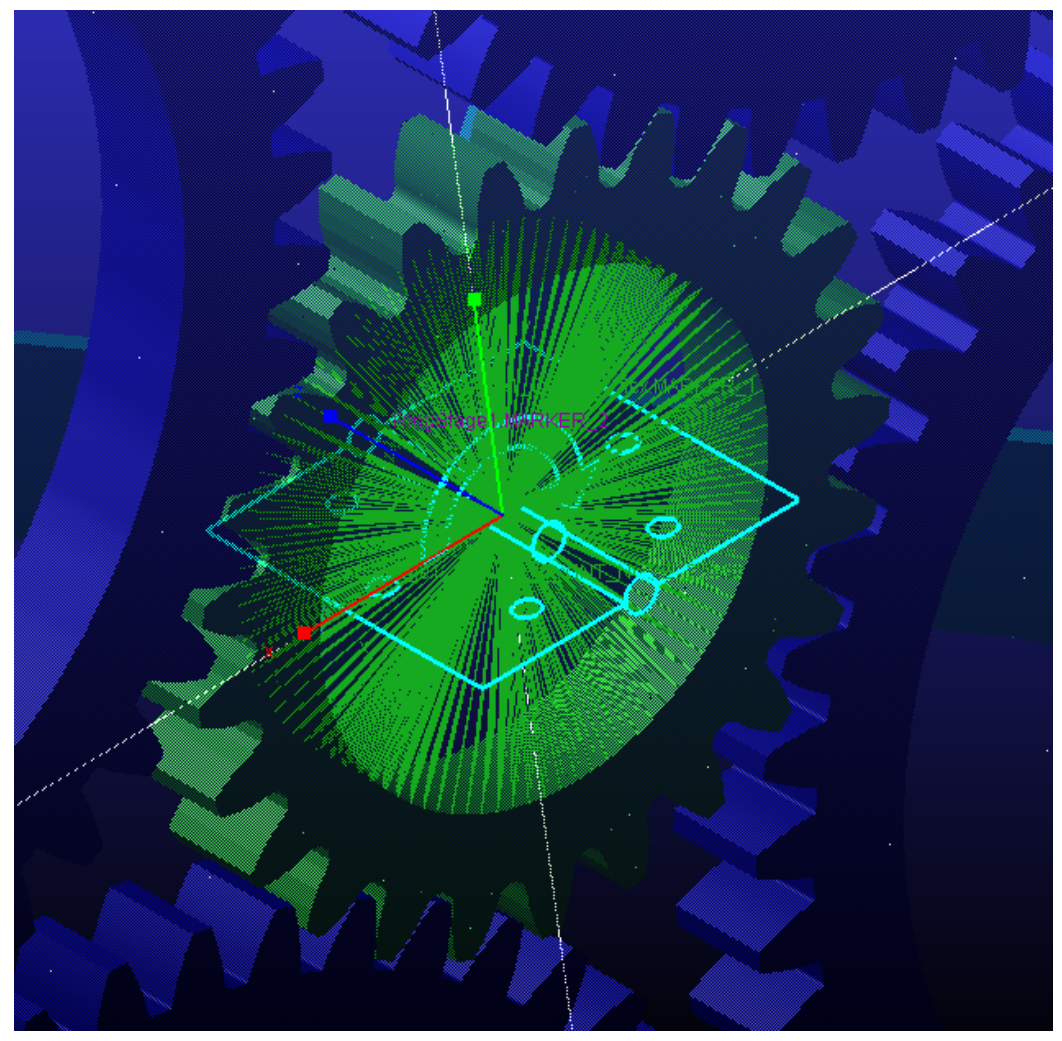


Create Contact

\begin{tabular}{|l|l|}
\cline { 2 - 2 } Contact Name & AssemblyModel_CONTACT_1 \\
\hline Contact Type & Flex Body to Solid \\
\hline I Flexible Body & SunStage1_Default_flex \\
J Solid & SOLID1 \\
& \\
\hline Force Display & Red \\
\hline Normal Force & Impact \\
\hline Stiffness & $1.0 \mathrm{E}+05$ \\
Force Exponent & 2.2 \\
Damping & 10.0 \\
Penetration Depth & 0.1 \\
\hline
\end{tabular}

\begin{tabular}{|c|c|c|c|}
\hline Friction Force & None & & $\checkmark$ \\
\hline & OK & Apply & Close \\
\hline
\end{tabular}

For a simple angular velocity input and resistive torque. Add motion to the orange gear by selecting the revolute joint associated with it and set the type to "Velocity" and function to "1.76" radians/second. Set initial velocity about $\mathrm{z}$ axis on the flexible body to 0 . Add a resistive torque to the appropriate torque. To add a contact force between the two gears. Click on "Create a Contact" in the Special Forces section at the top ribbon. In the contact menu, select the Contact Type text box and change from Solid to Solid to "Flex Body to Solid". If this is not set, the flexible body cannot be selected. This is required to be specified since ADAMS Solver is capable of using 2 different codes: FORTRAN and $\mathrm{C}++$. Fortran is not able to handle contact that is more than a point to a Plane. This will indicate that $\mathrm{C}++$ Solver will be used. In the text field for "I Flexible Body" right click and select pick and click on the Flexible body. Repeat this for the J Solid and select the orange output gear. Specify the Impact model Stiffnes, Force Exponent, Damping, and Pentration Depth. 
Max Prin. Stress (MPa)
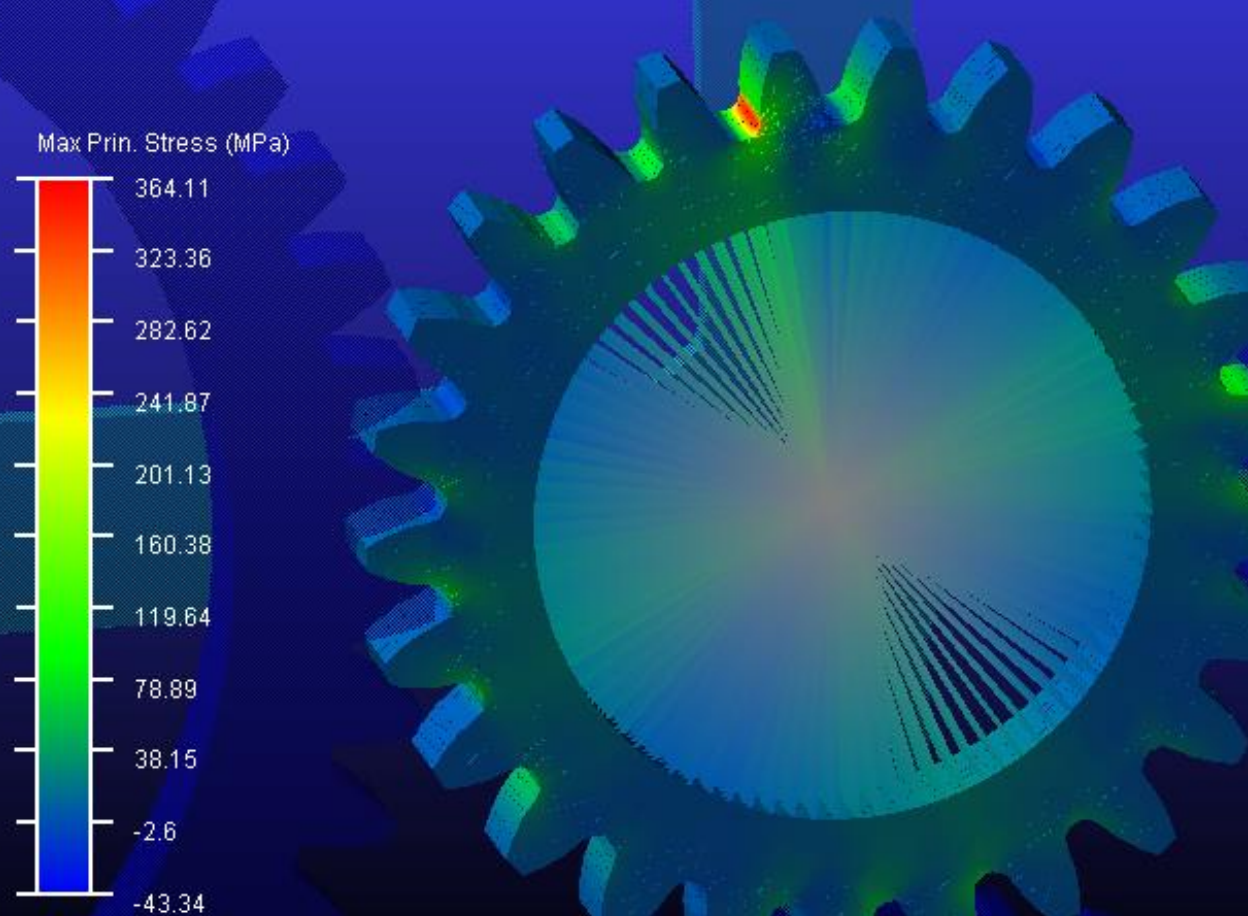

3

$-43.34$

Once all forces, motion, joints are complete. Run the simulation, wait for it to finish and open the plotting option. In the main ADAMS window go to the Tools drop down menu and select "Plugin Manager...". Check the box to load "ADAMS Durability" and click "OK". Go back to the plot window, right click the main plot area and select "Load Animation" at the bottom section select "Contour Plots". Here there are options for what type of stress we want to display on the animation and the ability to adjust the scale for those stress options. 
This is an example Contour Plot for Max Principal Stress on the Flexible body.

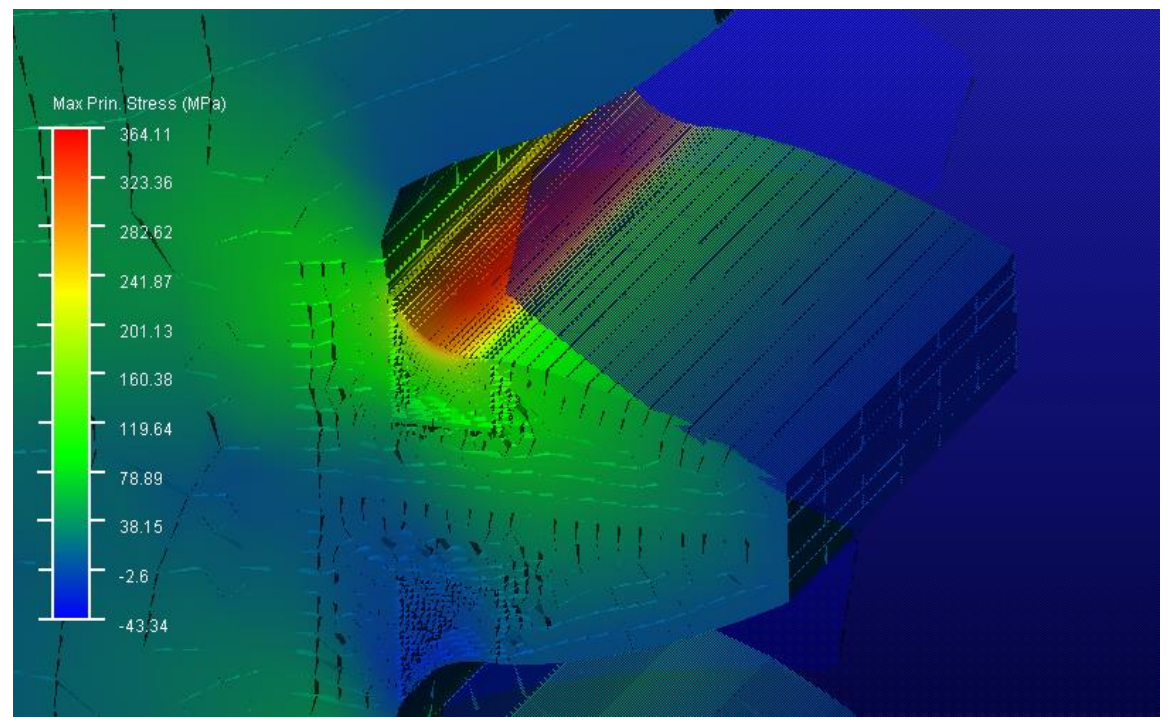

Note that the mesh made for this gear is quite course due to the machines ability to process a large number of output nodes and a fine mesh on the gear teeth in Patran. This will be a common issue with gear meshing where a finer mesh on the gear teeth will take large amounts of computer processing power to run a full analysis. 


\section{APPENDIX B - VIEW FLEX TUTORIAL}

\section{MSC Adams Flexible Body Creation for a Fix Axis Output Gear in a Planetary Gear System Assembly: A Tutorial}

Tananant (Mel) Boonya-ananta

tboonyaa@calpoly.edu

Mechanical Engineering Department

California Polytechnic State University

San Luis Obispo, CA 93407 


\section{Tutorial.}

Prepare a model using Solidworks or similar CAD programs as a Parasolid file (.x_t or .x_b). For this tutorial a file has been prepared as Stage3NoCrack2.x_t.

Start by opening MSC ADAMS View.

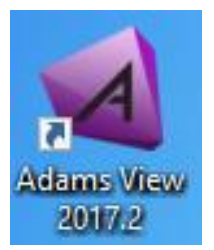

Create a new directory with the parasolid file and choose that as the working directory for ADAMS.

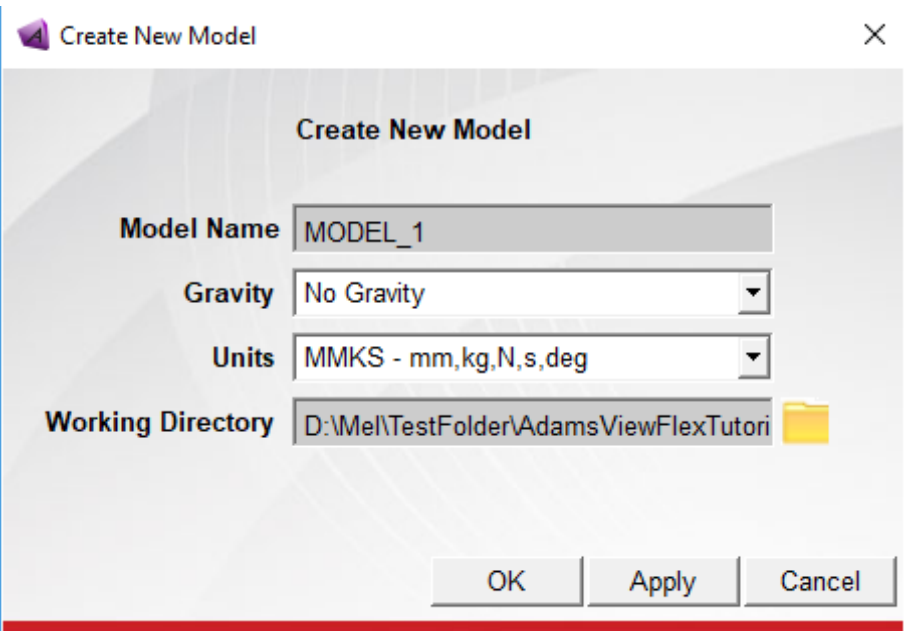

Once the model has been created, navigate File > Import. Select Parasolid in the drop down menu under File Type. Right click the text box under File To Read and browse for the parasolid file name Stage3NoCrack.x_t. Assign a name to the model.

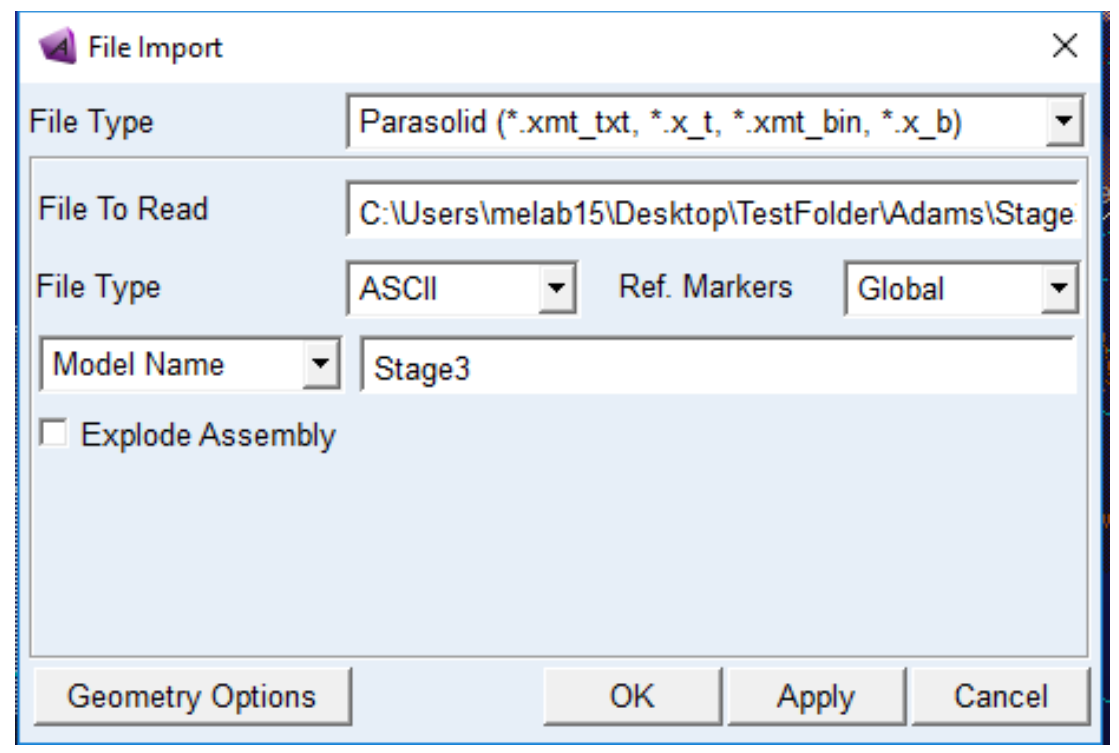

The imported model should look like this: 


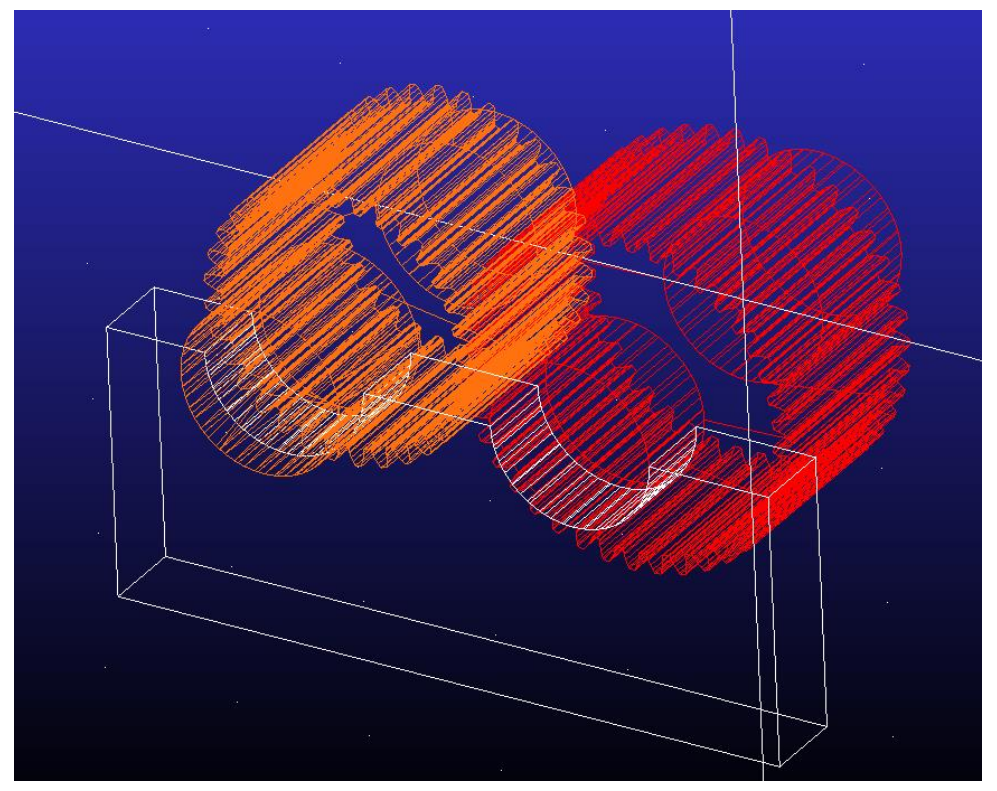

Switch from wireframe view to shaded model view:

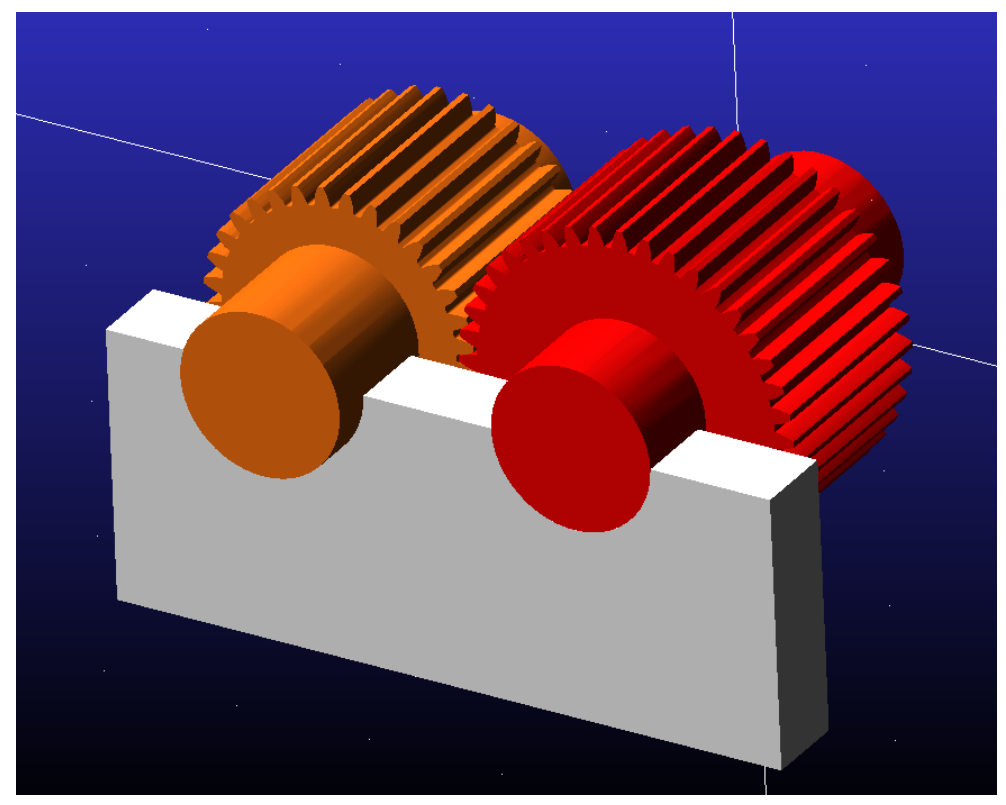


First define the mass properties of each body:

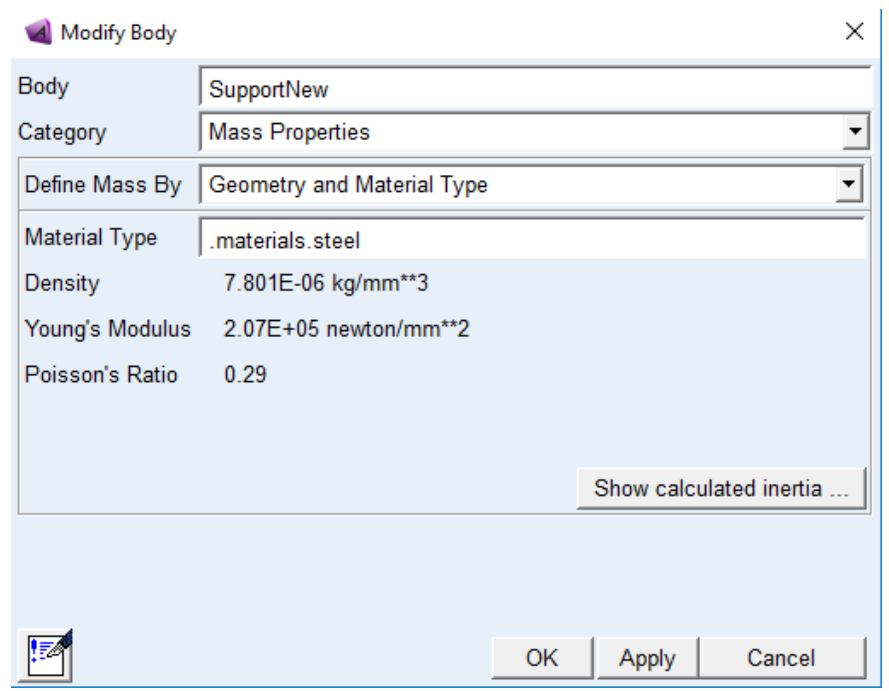

Add a marker to Ground at location $(0,0,0)$. This will provide a good locating feature for joints.

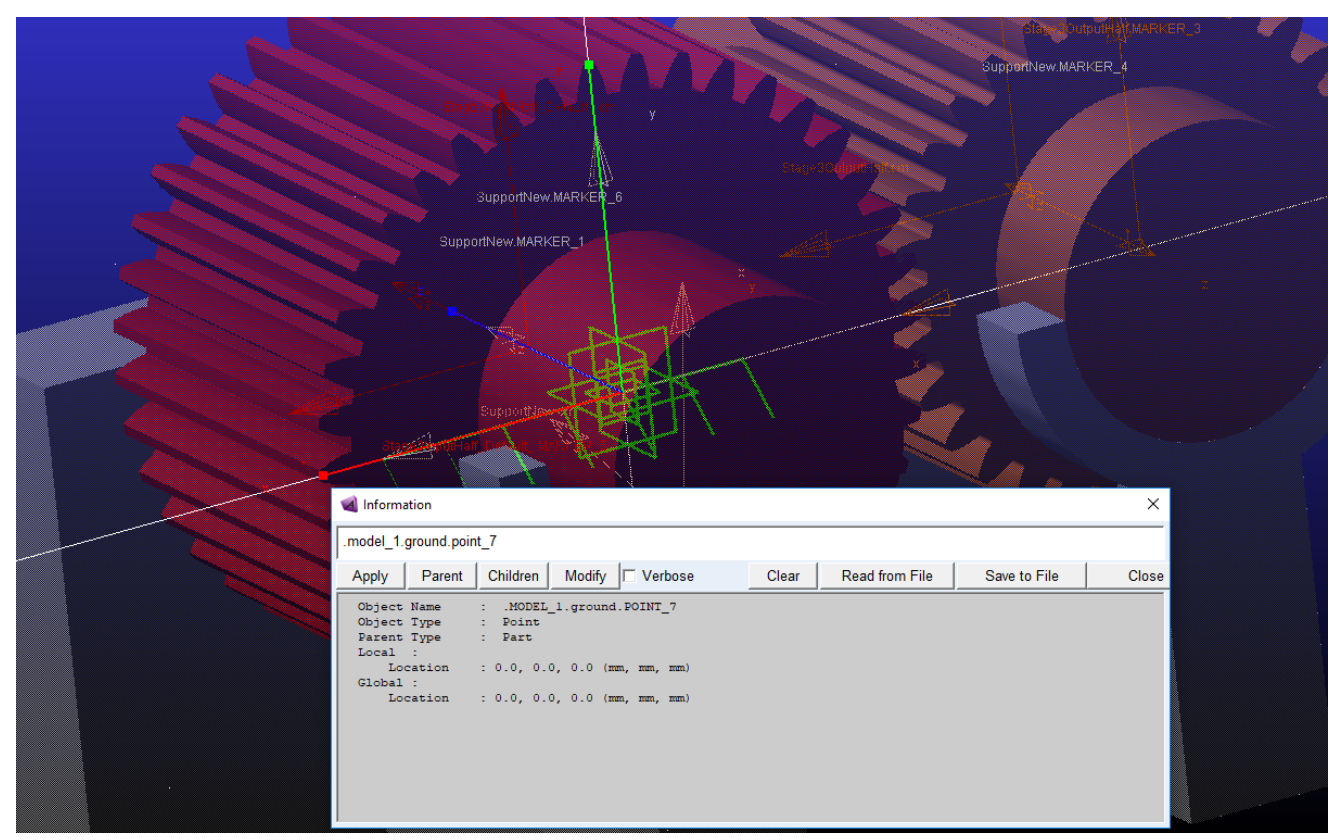


Add the appropriate joint for the parts in the assembly, this will include a fixed joint for the support to ground, a revolute joint between the output gear (orange) to the support at the center of the output gear shaft, and another joint on the input gear (red) to the support at the center of the gear shaft.

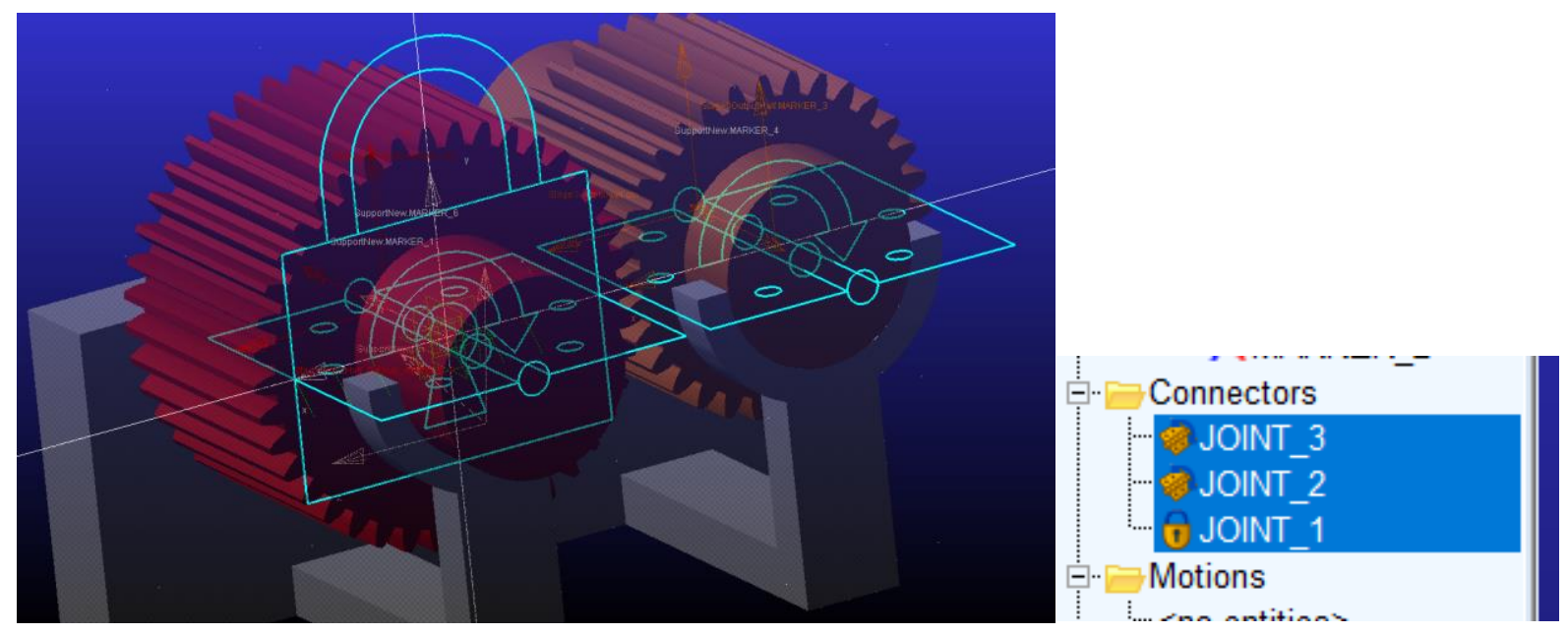

The input gear (red) will be the part which will be made flexible. The addition of this joint defines a constraint location for the gear.

To make the part flexible using ViewFlex, navigate to the Body tab.

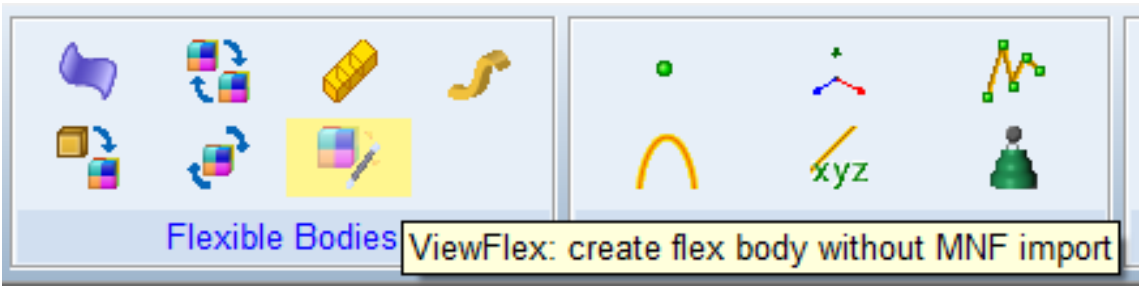

Select Create A Flexible Body Without an .mnf file. 
This will bring up a menu. Define the mesh properties and the number of Normal Modes required for the body.

ViewFlex - Create

Part to be meshed

$\longdiv { \text { MODEL_1.Stage3InputHa } }$

Load AFI File

Material

Number of Modes

MODEL_1.steel

40

「Manual Replace

FlexBody Type C Extrusion C Geometry $C$ Import Mesh

FlexBody Name $\quad \longdiv { \text { Stage3InputHalf Default fl } }$

- Mesh/Properties $C$ Attachments Mesh preview

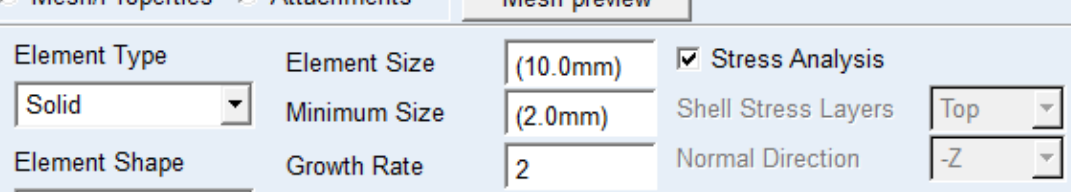

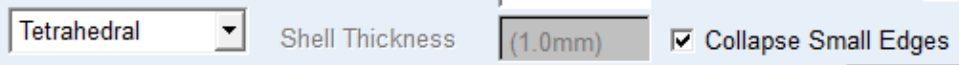

Element Order Angle Per Element $\longdiv { ( 4 5 \mathrm { deg } ) }$ Edge Tolerance $(0.0 \mathrm{~mm})$

Quadratic $\quad \rightarrow$

Element Specification MSN Based Refinement $r$ ON $\bullet$ OFF

Size

$\checkmark$

Curvature Based Scaling $C$ ON $\bullet$ OFF

Edge Shape

Curved $\quad \longrightarrow$

国过

Color $\longdiv { \text { red } \quad \square }$

$\ulcorner$ Advanced Options

$\times$

Once the properties are in created, Click OK. Note that this is a coarse mesh. The quality of the mesh is dependent on the computing power of the computer. Higher end computers will be able to create a fine mesh due to the available computing power.

Message Window...

Storing Solid Mesh Data...

Creating Mesh Preview for Solid in progress...

Nodes $=2406$, Element $5=4808$

Autodetecting Attachment Nodes...

Storing Solid Mesh Data.

Creating Geometry Mesh Flex Body in progress..

Replacing rigid body with flexible body...

Replace process successfully completed.

Settings...

Clear $\quad$ Close

Wait for the message window to display to complete. This will create multiple new files as a result of creating a flexible boy in the working directory. 


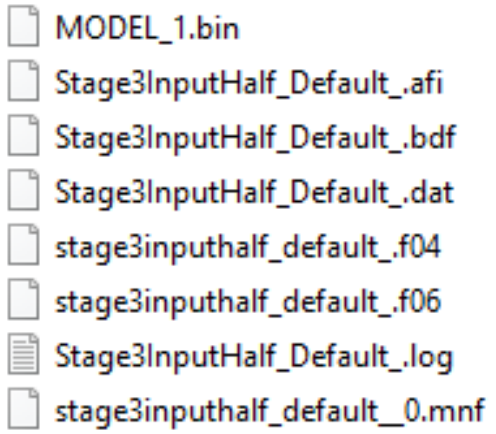

$\begin{array}{llr}11 / 22 / 201711: 51 \ldots & \text { BIN File } & 31,189 \text { KB } \\ 11 / 22 / 201711: 45 \ldots & \text { AFI File } & 3 \mathrm{~KB} \\ 11 / 22 / 201711: 45 \ldots & \text { BDF File } & 501 \mathrm{~KB} \\ 11 / 22 / 201711: 45 \ldots & \text { DAT File } & 501 \mathrm{~KB} \\ 11 / 22 / 201711: 46 \ldots & \text { F04 File } & 254 \mathrm{~KB} \\ 11 / 22 / 201711: 46 \ldots & \text { F06 File } & 73 \mathrm{~KB} \\ 11 / 22 / 201711: 46 \ldots & \text { Text Document } & 1 \mathrm{~KB} \\ 11 / 22 / 201711: 45 \ldots & \text { MNF File } & 11,961 \mathrm{~KB}\end{array}$

The two main files to be aware of in this directory is the .f06 file and the .mnf file. The fo6 file is the file that contains any types of error that would come about in the flexible body creation process. This file can be open in NotePad or NotePad++ and viewed to determine issues in the process. The .mnf file is the Modal Neutral File which contains the stress analysis information that allows ADAMS to perform the stress analysis under a dynamic simulation.

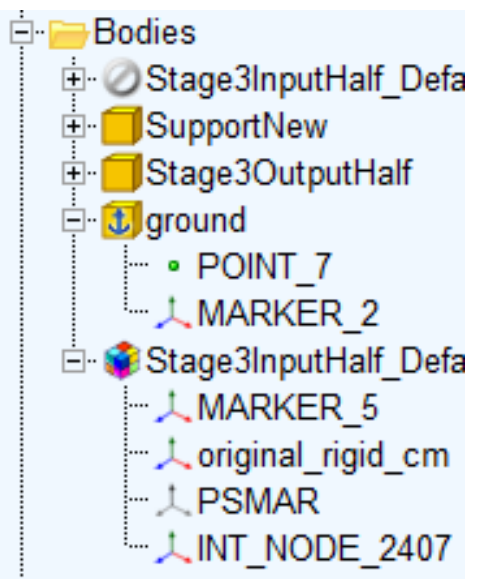

The model tree will show that original part as suppressed and hidden and replace it with a new flexible body as denoted by the rainbow color cube in the left side.

Navigate to the Settings menu and click on Units. Select Radians for Angle. 


\begin{tabular}{|c|c|c|c|}
\hline \multicolumn{4}{|c|}{ A Units Settings } \\
\hline Length & Millir & eter & \\
\hline Mass & Kilog & $\mathrm{am}$ & \\
\hline Force & Newt & & \\
\hline Time & Seco & & \\
\hline Angle & Radi & & \\
\hline Frequency & Hertz & & \\
\hline MMKS & MKS & CGS & IPS \\
\hline & OK & & incel \\
\hline
\end{tabular}

Select the Motions Ribbon and Click on Rotational Joint under Joint Motions

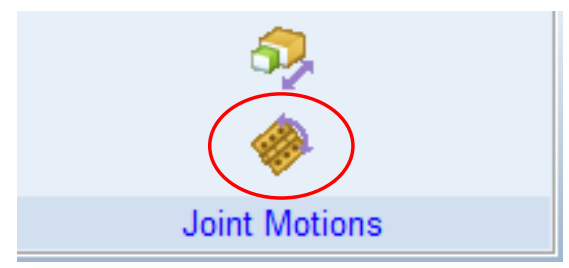

Pick Joint 3 as the joint of interest for the input motion.

\begin{tabular}{|c|c|c|}
\hline A Joint Motion & & X \\
\hline Name & MOTION_ & \\
\hline Joint & JOINT_3 & \\
\hline Joint Type & revolute & \\
\hline Direction & Rotational & 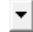 \\
\hline Define Using & Function & $\checkmark$ \\
\hline Function (time) & 110 & $\ldots$ \\
\hline Type & Velocity & - \\
\hline Displacement IC & & \\
\hline Velocity IC & & \\
\hline PEd & Apply & \\
\hline
\end{tabular}

Set the motion type to velocity and the function as 110 . This sets the input velocity to be $110 \mathrm{rad} / \mathrm{sec}$.

Add contact to the two gears. Navigate to Forces Ribbon and click Contact under Special Forces.

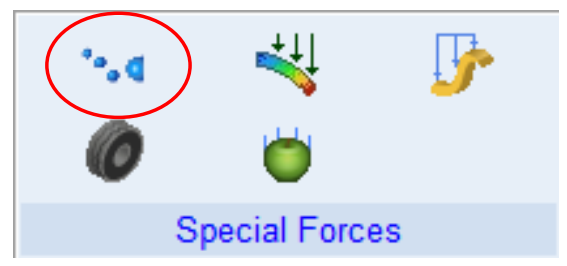


In the Create Contact menu. Change the type to be Flex Body to Solid. Select The new flexible body as I Flexible Body and the second gear (orange) under J Solid.

Uncheck Force Display.

Change the contact stiffness to 5.0E6, leave the force exponent as 2.2, change the damping to 10000, aAnd Penetration depth to 0.01 . Note that all these units contain millimeters as the primary unit of length.

\begin{tabular}{|l|l|}
\cline { 2 - 2 } Contact Name & MODEL_1.CONTACT_1 \\
\hline Contact Type & Flex Body to Solid \\
\hline $\begin{array}{ll}\text { I Flexible Body } \\
\text { J Solid }\end{array}$ & Stage3lnputHalf_Default__ \\
\cline { 2 - 2 } & SOLID2 \\
\hline$\Gamma$ Force Display & Red \\
\hline Normal Force & Impact \\
\hline Stiffness & $5.0 \mathrm{E}+06$ \\
\hline Force Exponent & 2.2 \\
\hline Damping & 10000 \\
\hline Penetration Depth & .01 \\
\hline & \\
\hline Friction Force & None \\
\hline & OK \\
\hline
\end{tabular}

In order to set up the measurement, three markers must be added to the model. One at the origin, another to create an axis to measure from, and the last to track the rotating point.

First, navigate back to the Bodies Ribbon and select Marker under the Construction section. Add the marker to Ground and select the origin. Once added, right click the new marker (Marker_10) and click Info. This will show the coordinates of the marker to make sure that it is one $(0,0,0)$.

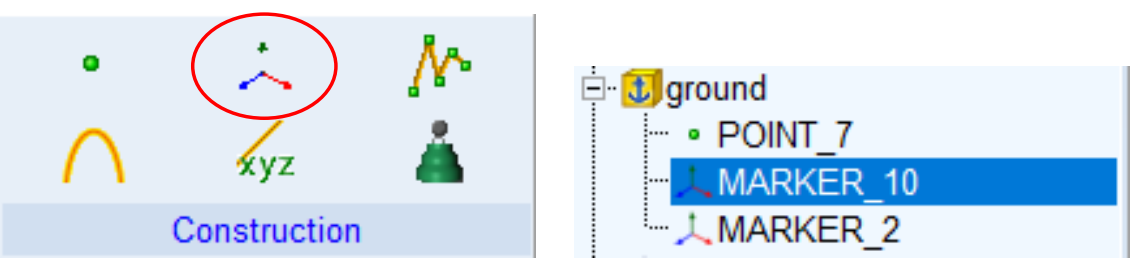


.model_1.ground.marker_10

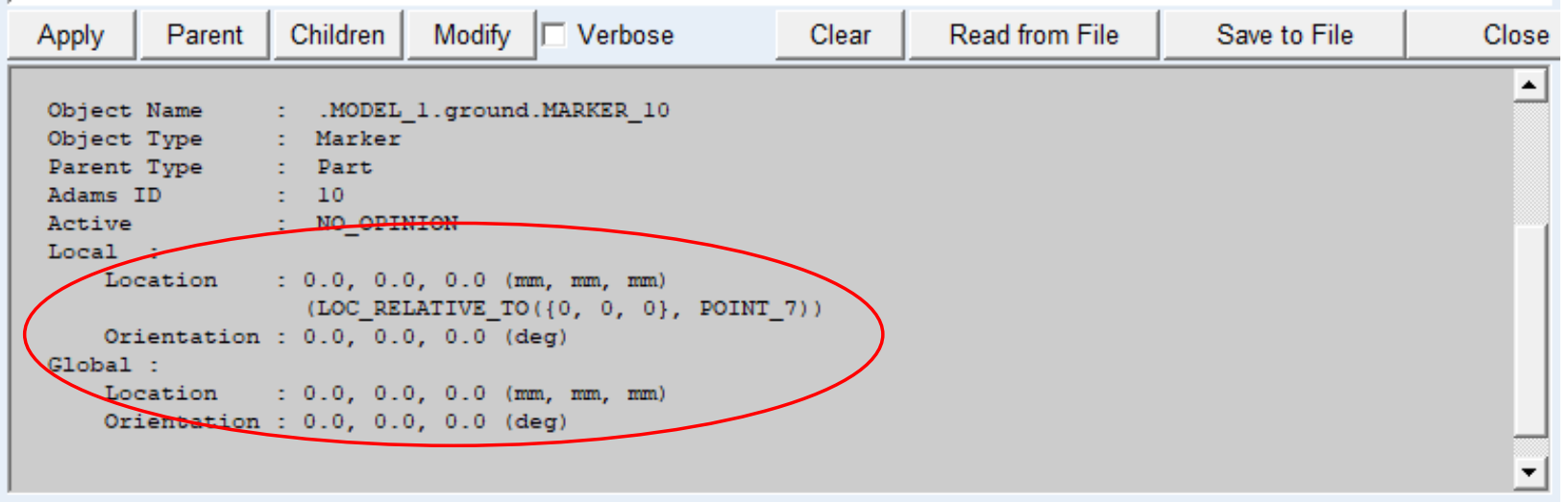

\section{Rename this Marker to AngleMeas2.}

Create another Marker on Ground on the XY Plane that is horizontal at location $(-50,0,0)$ and rename this to AngleMeas1. Hiding the gear body will make this marker easier to place without accidentally placing it on a specific node on the gear.

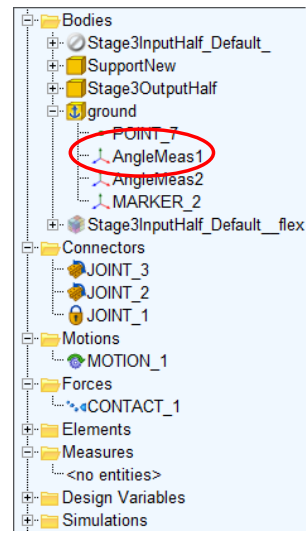

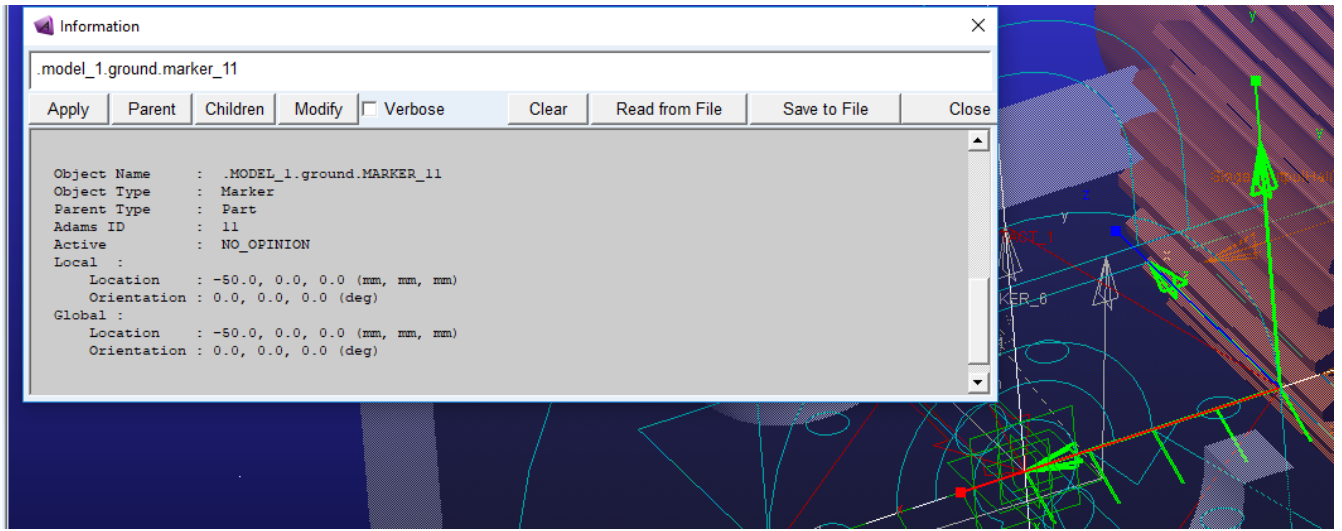

Create another marker on the flexible gear at the same location as AngleMeas1 marker but on the gear body instead of ground.

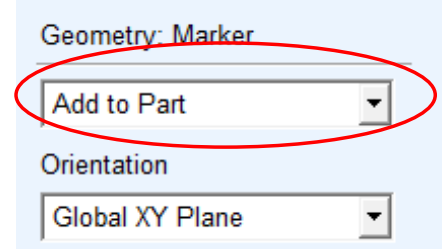


This marker will sometimes be added slightly shifted from $(-50,0,0)$. Right click the marker (Marker_12) and click Modify. Change the coordinates to $(-50,0,0)$. Click OK.

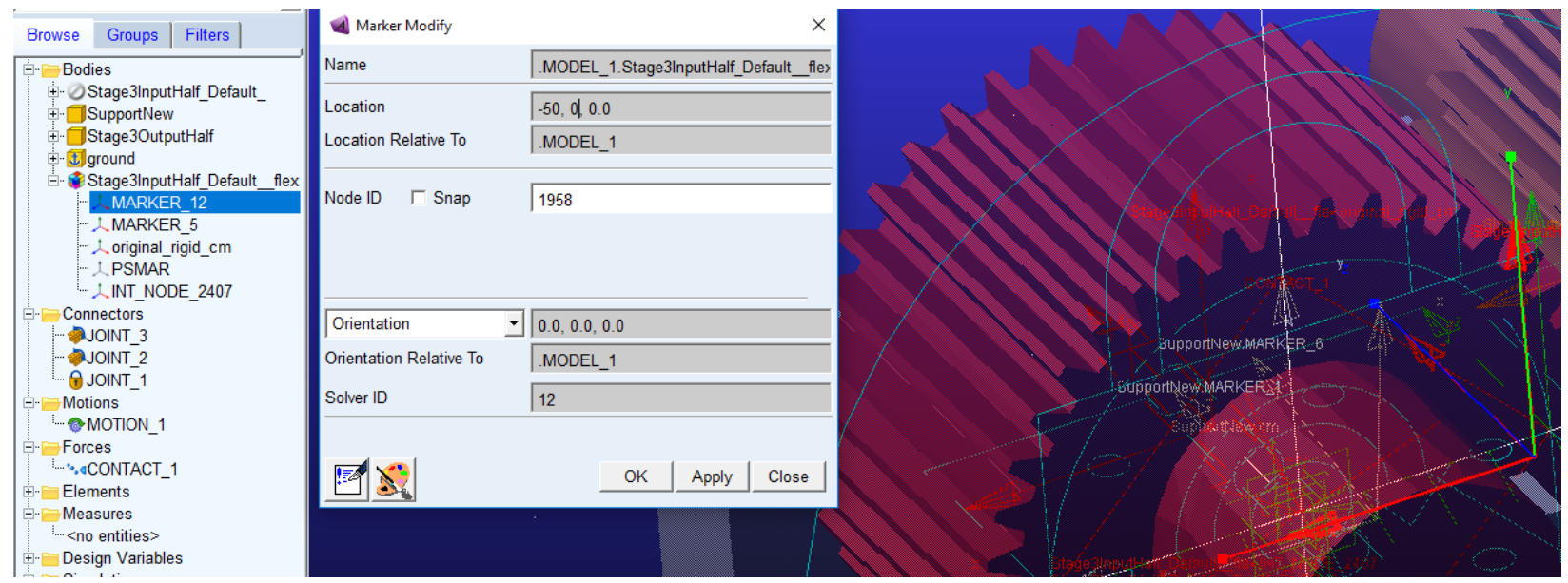

Rename to AngleMeas3.

Navigate to the Design Exploration Ribbon. Under the Measures Section select Create a new Angle Measurement.

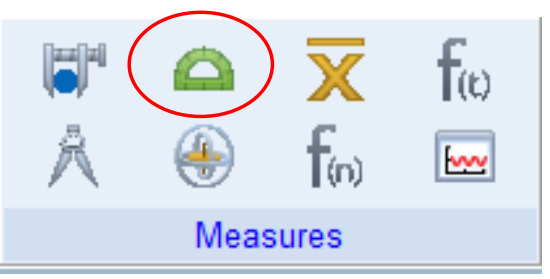

Click on Advanced to get a new popup window. In this window, the three markers that were just created will be selected to define the angle measurement.

Select AngleMeas1 under First Marker. AngleMeas2 for the Middle Marker. AngleMeas3 for the Last Marker.

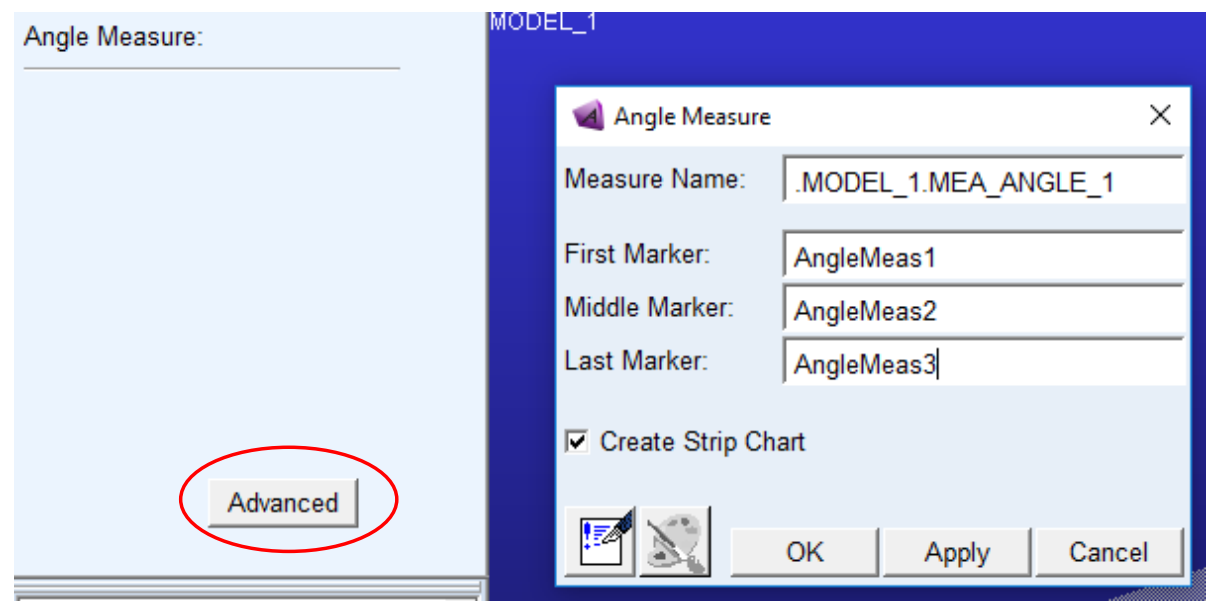

Click OK. A new window will pop up to show the angle measurement over time. Hit escape to exit out of the angle measurement menu. 


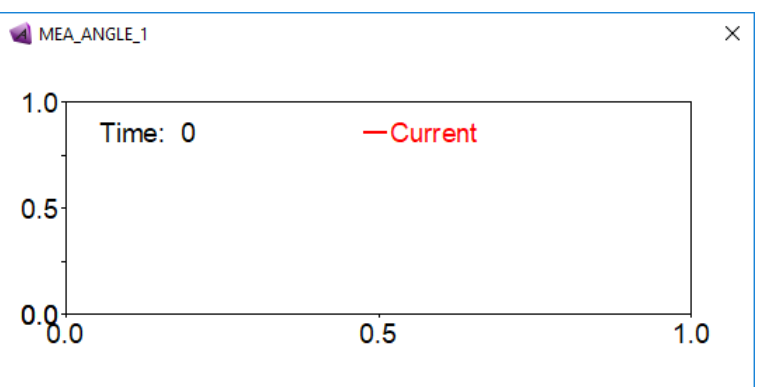

Move to the Simulation Ribbon and Select Run an Interactive Simulation under the Simulate Section. A new pop up window will appear with simulation parameters. Set the simulation End Time to 0.057 seconds and use 200 Steps. 0.57 is the amount of time that the Red Gear will complete 1 full rotation.

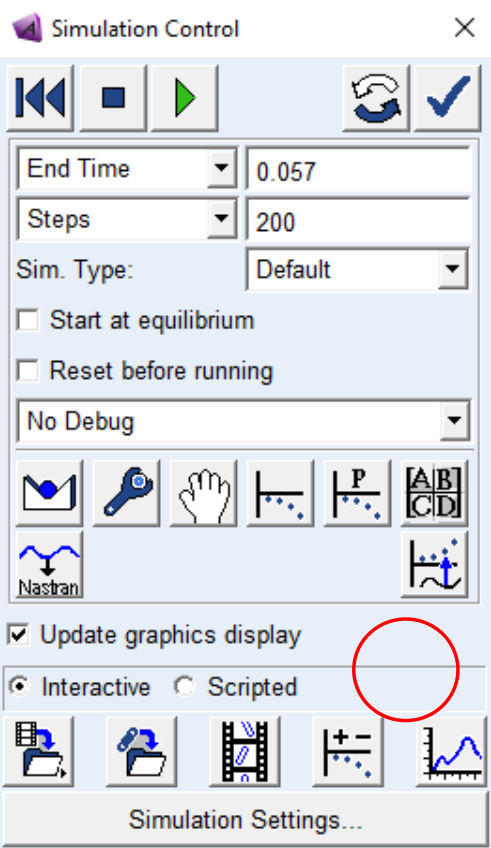

Click the Green Arrow to Run the simulation. Select Plotting once the simulation has completed. 
First, plot the angular velocity about the $\mathrm{z}$ axis of both the gears to see that it produces the correct ratio. This is done by selecting Objects for Source, the gear for Object, angular velocity for Characteristic, and $\mathrm{z}$ as the component then Add Curves.

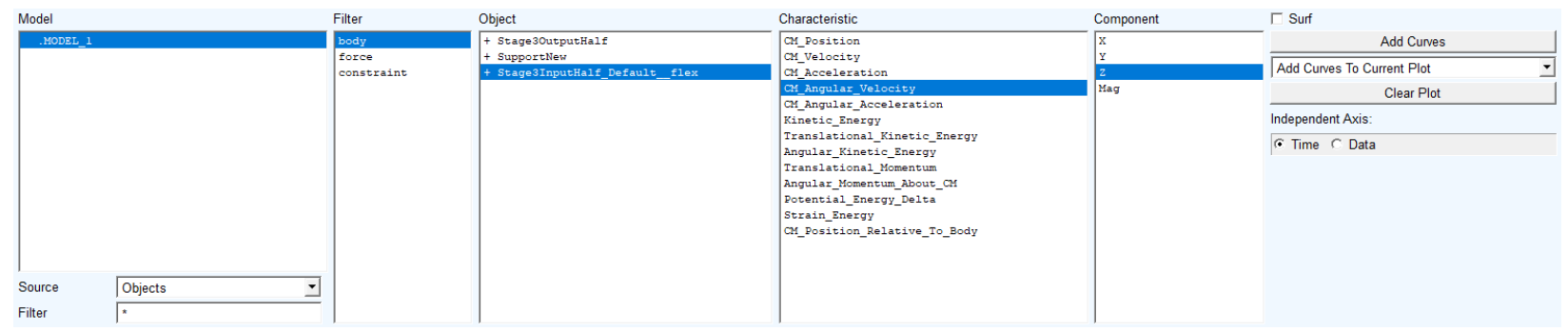

The Plot of these two components should look like this:

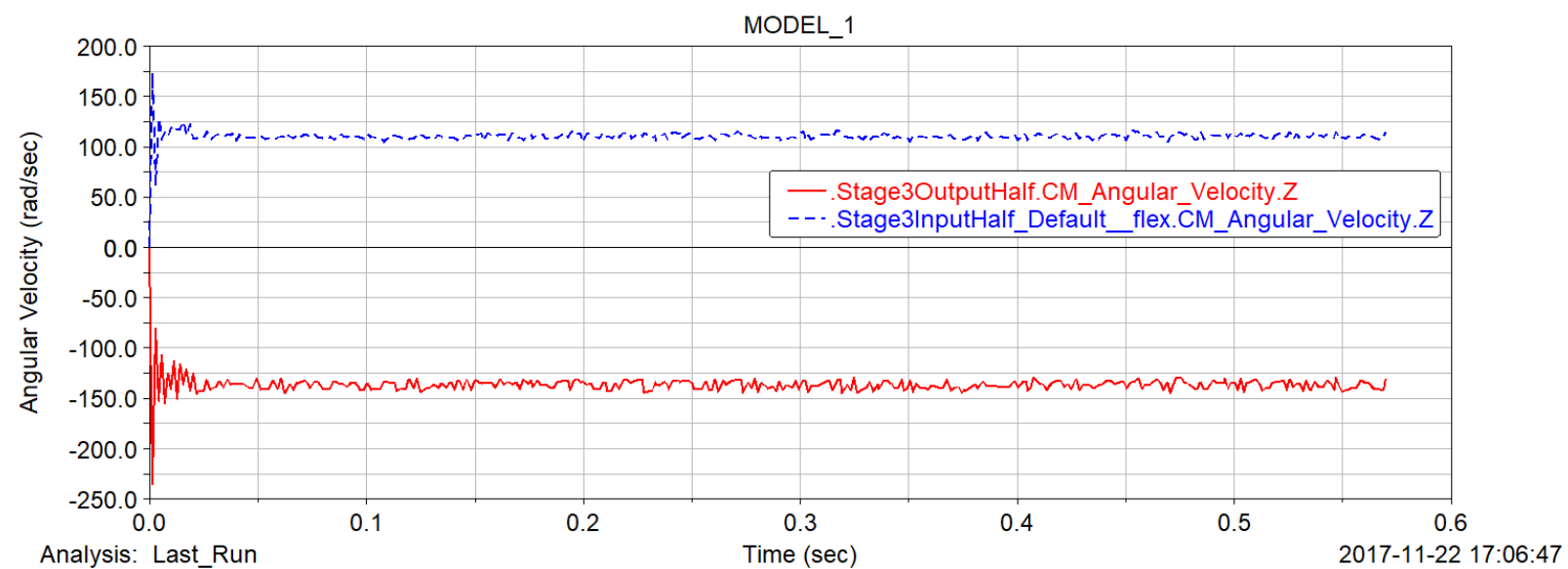

The input is the blue curve and the output is the red curve. The input velocity should be $110 \mathrm{rad} / \mathrm{sec}$ and the output should be around 137.5. This is representative of a 1.25 gear ratio.

In order the plot the tooth stress, the nodes must first be identified. To identify the Nodes that stress will be measured from, under the Durability Menu at the top of the Plot Window select Nodal Plots... (If Durability is not there, go to Tools > Plugin > Manager and check Adams Durability under Yes in the Load Column).

A Compute Nodal Plot window will pop up. Under Flexible Body select the Flexible Gear. Then, in the Node to Add to List text box, right click and click Pick Flexbody Node.

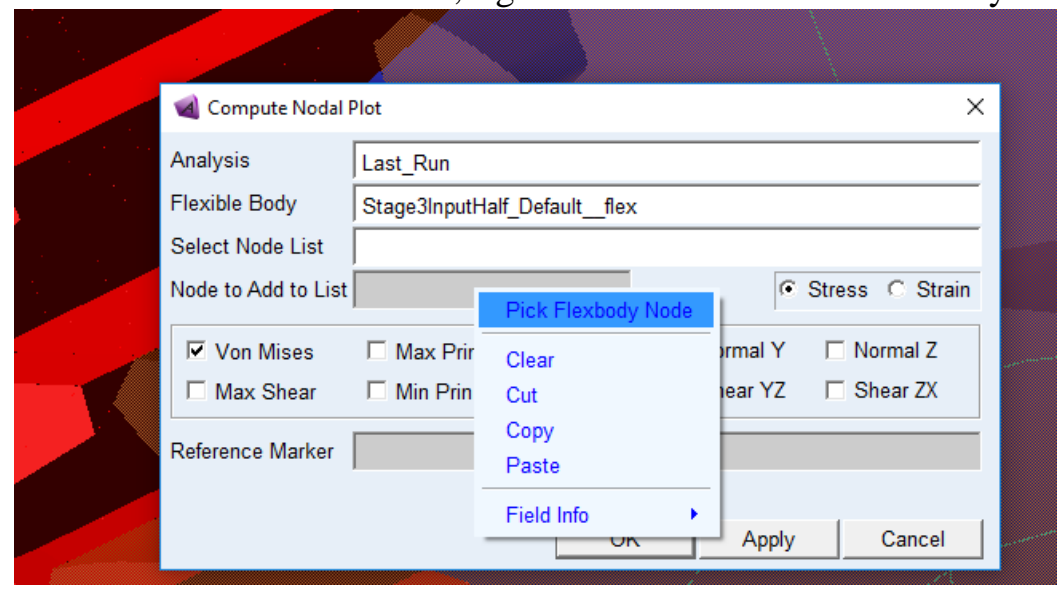


Mouse over the desired Node and not down the Node Number.

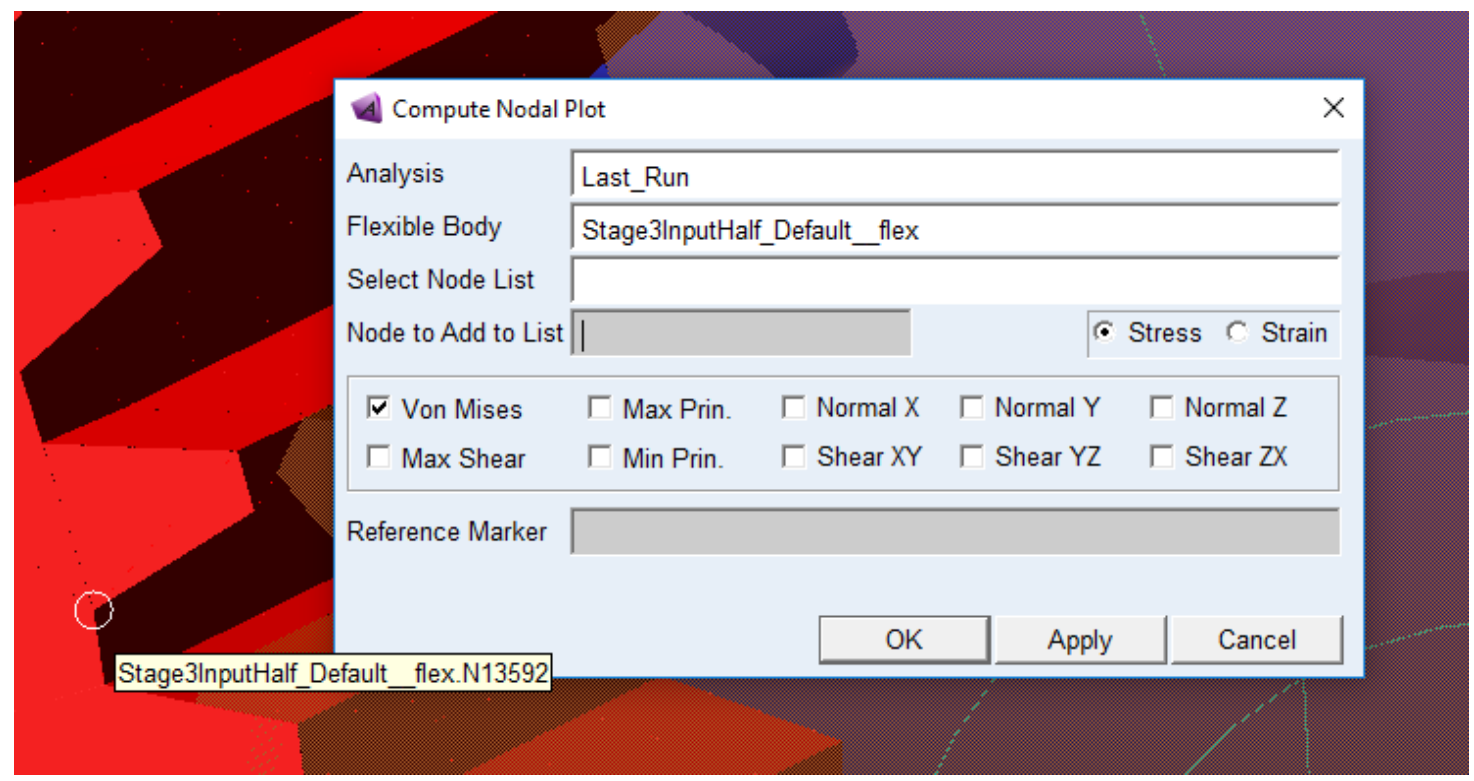

Copy the Node Number into the Select Node List. Here multiple nodes can be selected to analysis the stress at different nodes. Once added Click OK.

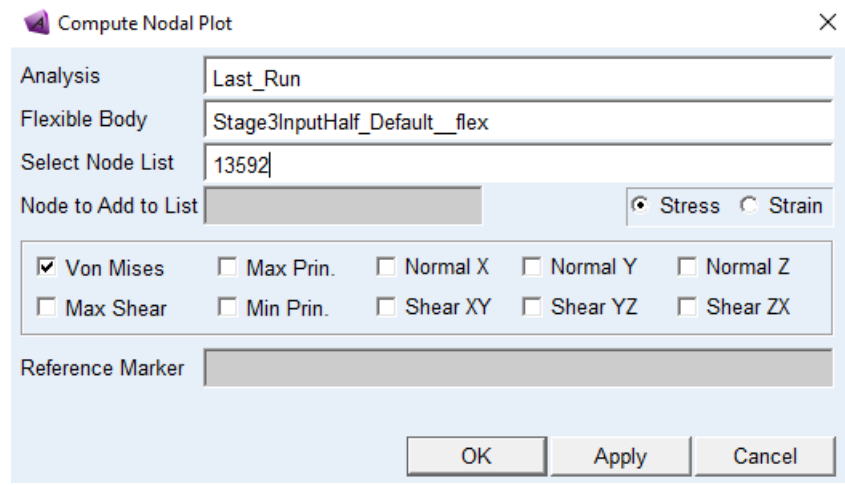


Clear the plot in the plot window by Clicking Clear Plot. In the bottom left corner, under Source select Results Sets, the stress of the added Node will appear under Result Set. Note that if the stress does not show up change to Measure or Objects then back to Results Set.
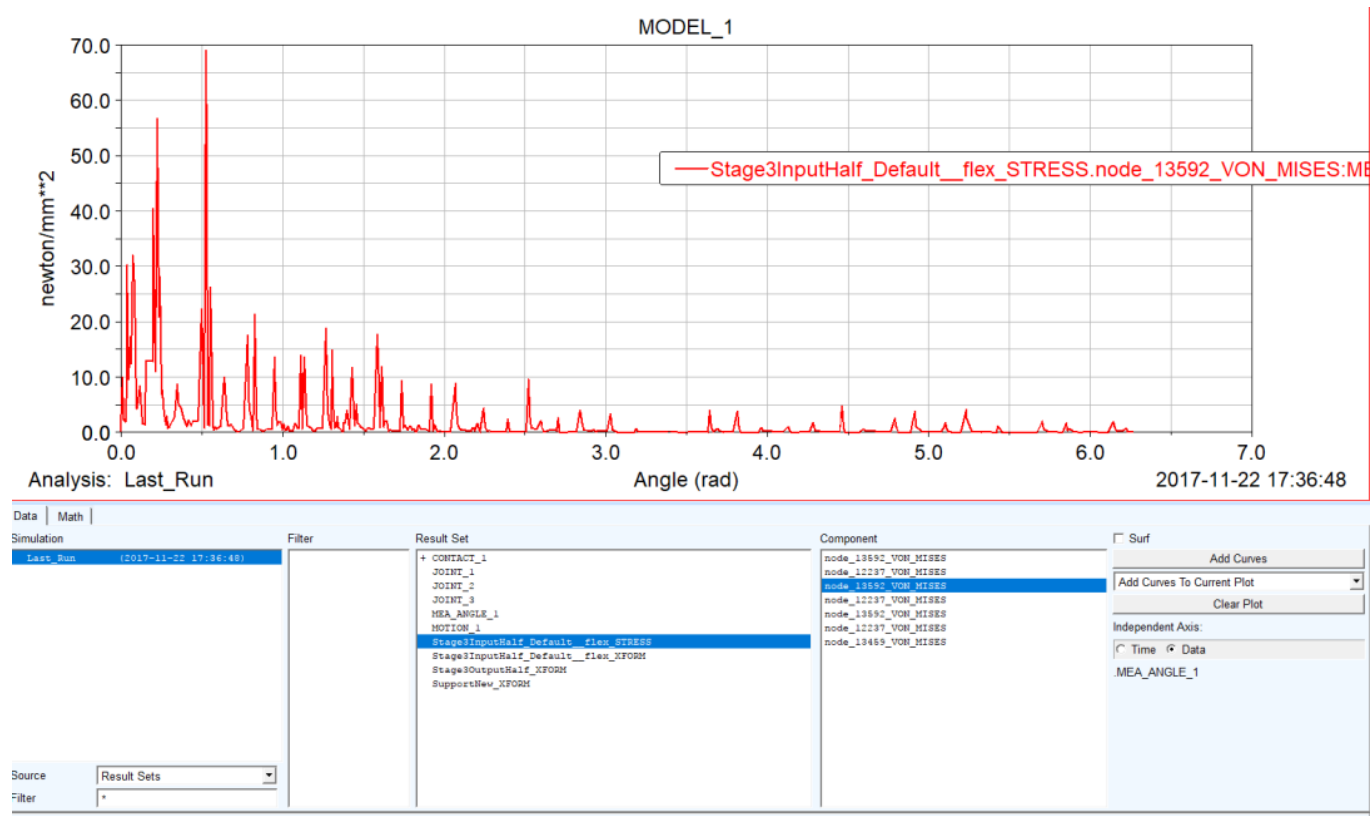